\title{
Differential Calculus of Hochschild Pairs for Infinity-Categories
}

Isamu IWANARI

Mathematical Institute, Tohoku University, 6-3 Aramakiaza, Sendai, Miyagi, 980-8578, Japan

E-mail: isamu.iwanari.a2@tohoku.ac.jp

Received February 25, 2020, in final form September 04, 2020; Published online October 02, 2020

https://doi.org/10.3842/SIGMA.2020.097

\begin{abstract}
In this paper, we provide a conceptual new construction of the algebraic structure on the pair of the Hochschild cohomology spectrum (cochain complex) and Hochschild homology spectrum, which is analogous to the structure of calculus on a manifold. This algebraic structure is encoded by a two-colored operad introduced by Kontsevich and Soibelman. We prove that for a stable idempotent-complete infinity-category, the pair of its Hochschild cohomology and homology spectra naturally admits the structure of algebra over the operad. Moreover, we prove a generalization to the equivariant context.
\end{abstract}

Key words: Hochschild cohomology; Hochschild homology; operad; $\infty$-category

2020 Mathematics Subject Classification: 16E40; 18N60; 18M60

To Kyoji Saito on his 7rth birthday

\section{Introduction}

Let $M$ be a smooth real manifold. Let $T_{M}^{\bullet}=\bigoplus_{p \geq 0} \wedge^{p} T_{M}$ and $\Omega_{M}^{\bullet}=\bigoplus_{q \geq 0} \Omega_{M}^{q}$ denote the graded vector space of multivector fields and differential forms on $M$, respectively. By convention, $T_{M}^{p}=\wedge^{p} T_{M}$ has homological degree $-p$ while $\Omega_{M}^{q}$ has homological degree $q$ : we adopt the reverse grading. There are several algebraic structures on the pair $\left(T_{M}^{\bullet}, \Omega_{M}^{\bullet}\right)$. The graded vector space $T_{M}^{\bullet}$ has a graded commutative (associative) product given by $\wedge$. Further, the shifted graded vector space $T_{M}^{\bullet+1}$ inherits the structure of a graded Lie algebra defined by the SchoutenNijenhuis bracket $[-,-]$. On the other hand, $\Omega_{M}^{\bullet}$ has the de Rham differential $\mathrm{d}_{\mathrm{DR}}$ (we do not consider the obvious graded commutative algebra structure on $\Omega_{M}^{\bullet}$ because it is irrelevant to the noncommutative context). Since $\Omega_{M}^{q}$ is the dual vector space of $T_{M}^{q}$, the contraction morphisms $T_{M}^{p} \otimes \Omega_{M}^{q} \rightarrow \Omega_{M}^{q-p}$ give rise to a $\left(T_{M}^{\bullet}, \wedge\right)$-module structure on $\Omega_{M}^{\bullet}$ :

$i:\left(T_{M}^{\bullet}, \wedge\right) \otimes \Omega_{M}^{\bullet} \rightarrow \Omega_{M}^{\bullet}$,

where we regard $\left(T_{M}^{\bullet}, \wedge\right)$ as the graded algebra determined by $\wedge$. The Lie derivative on $M$ defines a Lie algebra action of $\left(T_{M}^{\bullet+1},[-,-]\right)$ on $\Omega_{M}^{\bullet}$ :

$$
l: \quad\left(T_{M}^{\bullet+1},[-,-]\right) \otimes \Omega_{M}^{\bullet} \rightarrow \Omega_{M}^{\bullet}
$$

The tuple $\left(\wedge,[-,-], \mathrm{d}_{\mathrm{DR}}, i, l\right)$ constitutes fundamental calculus operations on the manifold $M$. These operations are subject under certain relations such as $\mathrm{d}_{\mathrm{DR}}^{2}=0$, the Cartan homotopy/magic formula, the compatibility between the Lie algebra action $l$ and the de Rham differential $\mathrm{d}_{\mathrm{DR}}$, and so on. If $X$ is a smooth algebraic variety over a field of characteristic

This paper is a contribution to the Special Issue on Primitive Forms and Related Topics in honor of Kyoji Saito for his 77th birthday. The full collection is available at https://www.emis.de/journals/SIGMA/Saito.html 
zero, the pair $\left(\mathcal{T}_{X}^{\bullet}, \Omega_{X}^{\bullet}\right)$ of sheaves of multivector fields (given by the exterior products of the tangent sheaf) and differential forms admit such an algebraic structure of calculus.

Let us shift our interest to noncommutative algebraic geometry in which stable $\infty$-categories, (pretriangulated) differential graded (dg) categories or the likes play the roles of fundamental geometric objects. From the Hochschild-Kostant-Rosenberg theorem, an analogue of $\left(\mathcal{T}_{X}^{\bullet}, \Omega_{X}^{\bullet}\right)$ is the Hochschild pair, that is, the pair of Hochschild cohomology (cochain complex) and Hochschild homology (chain complex). In [23, Section 11.2], Kontsevich and Soibelman introduced a two-colored topological operad which we shall refer to as the Kontsevich-Soibelman operad and denote here by KS. It can be used to encode all the structures on the Hochschild pair, which are analogous to the structure of calculus. The operad $\mathbf{K S}$ generalizes the little 2-disks (cubes) operad, i.e., the $\mathbf{E}_{2}$-operad. It contains two colors, $D$ and $C_{M}$, such that the full suboperad spanned by the color $D$ is the $\mathbf{E}_{2}$-operad. In [11], it was shown that a combinatorial dg operad $\mathcal{K} \mathcal{S}_{\text {comb }}$, which is a certain dg version of $\mathbf{K S}$, acts on the pair of the Hochschild cochain complex and the Hochschild chain complex of an associative algebra ( $A_{\infty}$-sense), and in [16] an algebraic structure on the Hochschild pair over KS was constructed for a ring spectrum by means of the Swiss-cheese operad conjecture [34].

In this paper, we provide a conceptual new construction of the structure of an algebra over KS that yields the following result (see Theorem 7.14):

Theorem 1.1. Let $R$ be a commutative ring spectrum. Let $\mathcal{C}$ be a small $R$-linear stable indempotent-complete $\infty$-category. Let $\mathcal{H H}^{\bullet}(\mathcal{C})$ be the Hochschild cohomology $R$-module spectrum and $\mathcal{H}_{\bullet}(\mathcal{C})$ the Hochschild homology $R$-module spectrum. Then the pair $\left(\mathcal{H} \mathcal{H}^{\bullet}(\mathcal{C}), \mathcal{H} \mathcal{H}_{\bullet}(\mathcal{C})\right)$ is promoted to an algebra over $\mathbf{K S}$, namely, it is a $\mathbf{K S}$-algebra in the $\infty$-category of $R$-module spectra $\operatorname{Mod}_{R}$.

The structure of the KS-algebra on $\left(\mathcal{H} \mathcal{H}^{\bullet}(\mathcal{C}), \mathcal{H} \mathcal{H} \bullet(\mathcal{C})\right)$ in Theorem 1.1 induces the action morphism $u: \mathcal{H}^{\bullet}(\mathcal{C}) \otimes \mathcal{H H}_{\bullet}(\mathcal{C}) \rightarrow \mathcal{H}_{\bullet}(\mathcal{C})$, which is a counterpart to $i$ in the aforementioned tuple. In the classical differential graded algebraic situation, we further prove that $u$ can be described by means of well-known algebraic constructions. Thus, $u$ can be considered a noncommutative contraction morphism, see Section 8, Theorem 8.2 and Proposition 8.8. In other words, the underlying morphism given by actions is an expected one. By considering Cartan's homotopy formula built in $\mathbf{K S}$, an analogue $L: \mathcal{H H}^{\bullet}(\mathcal{C})[1] \otimes \mathcal{H} \mathcal{H}_{\bullet}(\mathcal{C}) \rightarrow \mathcal{H} \mathcal{H}_{\bullet}(\mathcal{C})$ of the Lie derivative map $l$ is also an expected morphism, cf. Remark 8.10.

In Section 2, we briefly describe the idea and approach of our construction, which is based on a simple observation. To achieve this, we prove the following (see Corollary 4.21 for details):

Theorem 1.2. Let $\mathcal{M}^{\otimes}$ be a symmetric monoidal $\infty$-category such that it admits small colimits and the tensor product functor $\otimes: \mathcal{M} \times \mathcal{M} \rightarrow \mathcal{M}$ preserves small colimits separately in each variable. (The typical examples of $\mathcal{M}^{\otimes}$ we should keep in mind are the $\infty$-category of spectra, and the derived $\infty$-category of vector spaces.)

Let $\operatorname{Fun}\left(B S^{1}, \mathcal{M}\right)$ be the functor category from the classifying space $B S^{1}$ of the circle $S^{1}$ to $\mathcal{M}$, which inherits a pointwise symmetric monoidal structure from the structure on $\mathcal{M}^{\otimes}$. Namely, an object of Fun $\left(B S^{1}, \mathcal{M}\right)$ can be viewed as an object $M$ of $\mathcal{M}$ equipped with an $S^{1}$-action. Let $\operatorname{Alg}_{\mathbf{K S}}(\mathcal{M})$ be the $\infty$-category of $\mathbf{K S}$-algebras in $\mathcal{M}$. Let $\operatorname{Alg}_{\mathbf{E}_{2}}(\mathcal{M})$ be the $\infty$-category of $\mathbf{E}_{2^{-}}$ algebras (i.e., algebras over the little 2-disks operad) in $\mathcal{M} . \operatorname{Similarly,} \operatorname{Alg}_{\mathrm{As}}\left(\operatorname{Fun}\left(B S^{1}, \mathcal{M}\right)\right)$ denotes the $\infty$-category of associative algebras $\left(\mathbf{E}_{1}\right.$-algebras $)$ in $\operatorname{Fun}\left(B S^{1}, \mathcal{M}\right)$. We denote by $\operatorname{LMod}\left(\operatorname{Fun}\left(B S^{1}, \mathcal{M}\right)\right)$ the $\infty$-category of pairs $(A, M)$ such that $A \in \operatorname{Alg}_{\mathrm{As}}\left(\operatorname{Fun}\left(B S^{1}, \mathcal{M}\right)\right)$ and $M$ is a left $A$-module object in Fun $\left(B S^{1}, \mathcal{M}\right)$.

Then there exists a canonical equivalence of $\infty$-categories

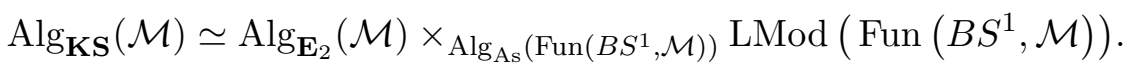

See Section 4 for the definition of the fiber product on the right-hand side. 
This result means that $\operatorname{Alg}_{\mathbf{E}_{2}}(\mathcal{M}), \operatorname{Alg}_{\mathrm{As}}\left(\operatorname{Fun}\left(B S^{1}, \mathcal{M}\right)\right)$ and $\operatorname{LMod}\left(\operatorname{Fun}\left(B S^{1}, \mathcal{M}\right)\right)$ form building blocks for KS-algebras. This allows us to describe the structure of a $\mathbf{K S}$-algebra as a collection of more elementary algebraic data involving associative algebras, left modules, circle actions, and $\mathbf{E}_{2}$-algebras. As for $\mathbf{E}_{2}$-algebras, thanks to Dunn additivity theorem for $\infty$-operads proved by Lurie [25], a canonical equivalence $\operatorname{Alg}_{\mathbf{E}_{2}}(\mathcal{M}) \simeq \operatorname{Alg}_{\mathrm{As}}\left(\operatorname{Alg}_{\mathrm{As}}(\mathcal{M})\right)$ exists. While we make use of Theorem 1.2 in the construction process, it would be generally useful in the theory of KS-algebras since the notion of $\mathbf{K S}$-algebras is complicated as is. For example, when $\mathcal{M}$ is the derived $\infty$-category $\mathcal{D}(k)$ of vector spaces over a field $k$ of characteristic zero, i.e., in the differential graded context, there is a quite elementary interpretation. One may take Fun $\left(B S^{1}, \mathcal{D}(k)\right)$ to be the $\infty$-category obtained from the category of mixed complexes in the sense of Kassel (see, e.g., [24]) by localizing quasi-isomorphisms. Therefore, an object of $\operatorname{Alg}_{\mathrm{As}}\left(\mathrm{Fun}\left(B S^{1}, \mathcal{D}(k)\right)\right)$ may be regarded as an associative algebra in the monoidal $(\infty-)$ category of mixed complexes. Objects of $\operatorname{LMod}\left(\operatorname{Fun}\left(B S^{1}, \mathcal{D}(k)\right)\right)$ can be described in a similar way. Moreover, $\operatorname{dg} \mathbf{E}_{2}$-operad is formal in characteristic zero.

As we will describe in the following section, our method consists of only natural procedures. In particular, by contrast with previous work, it does not involve/use complicated resolutions of operads or genuine chain complexes. Thus, we hope that our proposed approach can be applicable to other settings and generalizations such as $(\infty, n)$-categories. Indeed, the method allows us to prove an equivariant generalization of Theorem 1.1 (see Theorem 9.1):

Theorem 1.3. Let $G$ be a group object in the $\infty$-category $\mathcal{S}$ of spaces, that is, a group-like $\mathbf{E}_{1}$-space. Let $\mathcal{C}$ be a small $R$-linear stable idempotent-complete $\infty$-category. Suppose that $G$ acts on $\mathcal{C}$ (namely, it gives a left action). Then $\left(\mathcal{H} \mathcal{H}^{\bullet}(\mathcal{C}), \mathcal{H} \mathcal{H} \bullet(\mathcal{C})\right)$ is promoted to a $\mathbf{K S}$-algebra in $\operatorname{Fun}\left(B G, \operatorname{Mod}_{R}\right)$. Namely, $\left(\mathcal{H} \mathcal{H}^{\bullet}(\mathcal{C}), \mathcal{H} \mathcal{H}_{\bullet}(\mathcal{C})\right)$ is a $\mathbf{K S}$-algebra in $\operatorname{Mod}_{R}$, which comes equipped with a left action of $G$.

We would like to invite the reader's attention to the noteworthy features of our method:

- Our construction of the structure of an algebra on the Hochschild pair over KS starts with an $R$-linear stable $\infty$-category $\mathcal{C}$. Consequently, if we have an equivalence $\mathcal{C} \simeq \mathcal{C}^{\prime}$, we have a canonical equivalence $\left(\mathcal{H} \mathcal{H}^{\bullet}(\mathcal{C}), \mathcal{H} \mathcal{H} \bullet(\mathcal{C})\right) \simeq\left(\mathcal{H H}^{\bullet}\left(\mathcal{C}^{\prime}\right), \mathcal{H} \mathcal{H} \bullet\left(\mathcal{C}^{\prime}\right)\right)$ as algebras over $\mathbf{K S}$ (see Remark 9.4). Consider the situation that the associative algebra $A$ and $A^{\prime}$ in the $\infty$-category of $R$-modules have the equivalent module category $\operatorname{LMod}_{A}$ and $\operatorname{LMod}_{A^{\prime}}$, that is, $A$ and $A^{\prime}$ are (derived) Morita equivalent to one another. Here $\operatorname{LMod}_{A}$ and $\operatorname{LMod}_{A^{\prime}}$ denote the $\infty$-categories of left $A$-module spectra and left $A^{\prime}$-module spectra, respectively (cf. Section 3). Then $\operatorname{LMod}_{A} \simeq \operatorname{LMod}_{A^{\prime}}$ induces the canonical equivalence of Hochschild pairs as algebras over KS. In other words, our method provides a natural Morita invariant structure. This invariance has a fundamental importance in noncommutative geometry and is reasonable to expect, whereas the Morita invariant property of algebra structures in [11] and [16] remains an open problem. We would like to mention a recent work [1] in which the authors prove the Morita invariance of the calculus structure at the level of graded vector spaces of homology.

- The base ring can be any commutative ring spectrum. Note that it is not possible to use the dg operad $\mathcal{K} \mathcal{S}_{\text {comb }}$ in the generalization to the "spectral" setting.

- As stated Theorem 1.3, our functorial method allows us to generalize to equivariant situations. The $\infty$-categories with group actions naturally appear in future applications (see below).

- As revealed the outline in Section 2, our construction works well not with algebras but with $\infty$-categories. Thus, even when one is ultimately interested in algebras, it is important to consider the $\infty$-category of their modules. 
We would like to view our results from the perspective of noncommutative algebraic geometry. As mentioned above, the notion of KS-algebra structures is a counterpart to the calculus on manifolds. Thus, KS-algebras are central objects in "noncommutative calculus". We refer the reader to [12] and references therein for this point of view.

Recall the algebro-geometric interpretations of the Hochschild cohomology $\mathcal{H H}^{\bullet}(\mathcal{C})$ and Hochschild homology $\mathcal{H} \mathcal{H}_{\bullet}(\mathcal{C})$ for stable $\infty$-categories $\mathcal{C}$ or dg categories (somewhat more precisely, we assume that they are "linear" over a field of characteristic zero). The $\mathbf{E}_{2}$-algebra $\mathcal{H} \mathcal{H}^{\bullet}(\mathcal{C})$ governs the deformations theory of the stable $\infty$-category $\mathcal{C}$ in the derived geometric formulation. The Hochschild homology $\mathcal{H H}_{\bullet}(\mathcal{C})$ (more precisely, the Hochschild chain complex) inherits an $S^{1}$-action that corresponds to the Connes operator. Then $\mathcal{H} \mathcal{H} \bullet(\mathcal{C})$ with $S^{1}$-action gives rise to an analogue of the Hodge filtration: the pair of the negative cyclic homology and the periodic cyclic homology can be thought of as such a structure. (These algebraic structures are contained in the $\mathbf{K S}$-algebra $\left(\mathcal{H} \mathcal{H}^{\bullet}(\mathcal{C}), \mathcal{H} \mathcal{H}_{\bullet}(\mathcal{C})\right)$.) As revealed in [20] in the case of associative $(\mathrm{dg})$ algebras $A$, the action of $\mathcal{H H}^{\bullet}(A)$ on $\mathcal{H} \mathcal{H} \bullet(A)$ encoded by the $\mathbf{K S}$-algebra structure at the operadic level is a key algebraic datum that describes variations of the (analogue of) Hodge filtration along noncommutative (curved) deformations. Namely, the period map for noncommutative deformations (of an associative algebra) is controlled by the KSalgebra of the Hochschild cohomology and Hochschild homology. Therefore, the KS-algebra $\left(\mathcal{H} \mathcal{H}^{\bullet}(\mathcal{C}), \mathcal{H} \mathcal{H}_{\bullet}(\mathcal{C})\right)$ will provide a crucial algebraic input for the theory of period maps for deformations of the stable $\infty$-category $\mathcal{C}$. The significance of the generalization to the equivariant context (Theorem 1.3) will be seen when one comes to consider fruitful examples. The motivations partly come from mirror symmetry. For example, stable $\infty$-categories endowed with $S^{1}$-actions or some algebraic actions, that are interesting from the viewpoint of $S^{1}$-equivariant deformation theory, naturally appear from Landau-Ginzburg models in the context of matrix factorizations. Its equivariant deformations together with the associated Hodge structure should provide a categorification of the theory of Landau-Ginzburg models. As a second example, if $X$ is a sufficiently nice algebraic stack (more generally, a derived stack), one can consider the derived free loop space $L X=\operatorname{Map}\left(S^{1}, X\right)$ of $X$ (see, e.g., [5]). The stable $\infty$-category $\operatorname{Perf}(L X)$ of perfect complexes on $L X$ comes equipped with the natural $S^{1}$-action. Finally, we would also like to mention that main results in this paper form the basis for our recent work [19].

\section{Strategy and organization}

The purpose of this section is to outline the strategy of a construction of a KS-algebra structure on the pair of Hochschild cohomology and Hochschild homology and to give the brief organization of this paper. This section is something like the second part of introduction. We hope that the following outline will be helpful in understanding the content of the sequel. However, this section is independent with the rest of this paper so that the reader can skip it.

2.1. We will give an outline of the construction. Let $\mathcal{C}$ be a small stable $\infty$-category. If $\mathcal{C}$ is not idempotent-complete, we replace $\mathcal{C}$ by its idempotent-completion: we assume that $\mathcal{C}$ is idempotent-complete. While we work with stable $\infty$-categories over a commutative ring spectrum $R$ in the paper, for simplicity we here work with plain stable idempotent-complete $\infty$-categories (equivalently, we assume that $R$ is the sphere spectrum). We let $\mathcal{D}=\operatorname{Ind}(\mathcal{C})$ denote the Ind-category that is a compactly generated stable $\infty$-category. The $\infty$-category $\mathcal{D}$ is also equivalent to the functor category $\operatorname{Fun}^{\mathrm{ex}}\left(\mathcal{C}^{\mathrm{op}}, \mathrm{Sp}\right)$ of exact functors, where $\mathrm{Sp}$ is the stable $\infty$-category of spectra.

Let $\operatorname{Fun}^{\mathrm{L}}(\mathcal{D}, \mathcal{D})$ be the functor category from $\mathcal{D}$ to itself that consists of those functors which preserve small colimits. This functor category is a compactly generated stable $\infty$-category and inherits an associative monoidal structure given by the composition of functors. We de- 
note by $\mathcal{E} \operatorname{nd}(\mathcal{D})^{\otimes}$ the presentable stable $\infty$-category $\operatorname{Fun}^{\mathrm{L}}(\mathcal{D}, \mathcal{D})$ endowed with the (associative) monoidal structure.

Let $\operatorname{Alg}_{\mathrm{As}}(\mathrm{Sp})$ denote the $\infty$-category of associative ring spectra. Given $A \in \operatorname{Alg}_{\mathrm{As}}(\mathrm{Sp})$, we define $\operatorname{RMod}_{A}$ to be the $\infty$-category of right $A$-module spectra. Let us regard $A$ as a right $A$-module in an obvious way. Then there is an essentially unique colimit-preserving functor $p_{A}: \mathrm{Sp} \rightarrow \operatorname{RMod}_{A}$ which sends the sphere spectrum $\mathbb{S}$ in $\mathrm{Sp}$ to $A$. Let $\operatorname{Pr}_{\mathrm{St}}^{\mathrm{L}}$ be the $\infty$-category of presentable stable $\infty$-categories in which morphisms are colimit-preserving functors. The assignment $A \mapsto\left\{p_{A}: \mathrm{Sp} \rightarrow \operatorname{RMod}_{A}\right\}$ induces $I: \operatorname{Alg}_{\mathrm{As}}(\mathrm{Sp}) \rightarrow\left(\operatorname{Pr}_{\mathrm{St}}^{\mathrm{L}}\right)_{\mathrm{Sp} /}$. The right adjoint $E:\left(\operatorname{Pr}_{\mathrm{St}}^{\mathrm{L}}\right)_{\mathrm{Sp} /} \rightarrow \operatorname{Alg}_{\mathrm{As}}(\mathrm{Sp})$ of $I$ carries $p: \mathrm{Sp} \rightarrow \mathcal{P}$ in $\left(\operatorname{Pr}_{\mathrm{St}}^{\mathrm{L}}\right)_{\mathrm{Sp} /}$ to the endomorphism (associative) algebra $\operatorname{End}_{\mathcal{P}}(P)$, where $P$ is the image $p(\mathbb{S})$. Note that $\operatorname{Alg}_{\mathrm{As}}(\mathrm{Sp})$ has a natural symmetric monoidal structure whose tensor product is induced by the tensor product $A \otimes B$ in Sp. The $\infty$-category $\operatorname{Pr}_{\mathrm{St}}^{\mathrm{L}}$ also admits an appropriate symmetric monoidal structure in which $\mathrm{Sp}$ is a unit object, and $I: \operatorname{Alg}_{\mathrm{As}}(\mathrm{Sp}) \rightarrow\left(\mathrm{Pr}_{\mathrm{St}}^{\mathrm{L}}\right)_{\mathrm{Sp}}$, can be promoted to a symmetric monoidal functor. Applying $\operatorname{Alg}_{\mathrm{As}}(-)$ to $I: \operatorname{Alg}_{\mathrm{As}}(\mathrm{Sp}) \leftrightarrows\left(\operatorname{Pr}_{\mathrm{St}}^{\mathrm{L}}\right)_{\mathrm{Sp} /}: E$, we obtain

$$
\text { I: } \operatorname{Alg}_{\mathrm{E}_{2}}(\mathrm{Sp}) \simeq \operatorname{Alg}_{\mathrm{As}}\left(\operatorname{Alg}_{\mathrm{As}}(\mathrm{Sp})\right) \rightleftarrows \operatorname{Alg}_{\mathrm{As}}\left(\left(\operatorname{Pr}_{\mathrm{St}}^{\mathrm{L}}\right)_{\mathrm{Sp} /}\right) \simeq \operatorname{Alg}_{\mathrm{As}}\left(\operatorname{Pr}_{\mathrm{St}}^{\mathrm{L}}\right): E,
$$

where $\operatorname{Alg}_{\mathbf{E}_{2}}(\mathrm{Sp})$ is the $\infty$-category of $\mathbf{E}_{2}$-algebras, and $\operatorname{Alg}_{\mathbf{E}_{2}}(\mathrm{Sp}) \simeq \operatorname{Alg}_{\mathrm{As}}\left(\operatorname{Alg}_{\mathrm{As}}(\mathrm{Sp})\right)$ and the left equivalence follows from Dunn additivity theorem. Here we abuse notation by writing $I$ and $E$ for the induced functors. Let us regard $\operatorname{Alg}_{A s}\left(\operatorname{Pr}_{\mathrm{St}}^{\mathrm{L}}\right)$ as the $\infty$-category of monoidal presentable stable $\infty$-categories. The left adjoint sends an $\mathbf{E}_{2}$-algebra $A$ to the associative monoidal $\infty$-category $\operatorname{RMod}_{A}^{\otimes}$. The right adjoint carries a monoidal presentable stable $\infty$-category $\mathcal{M}^{\otimes}$ to the endomorphism spectrum $\operatorname{End}_{\mathcal{M}}\left(1_{\mathcal{M}}\right)$ of the unit object $1_{\mathcal{M}}$, endowed with an $\mathbf{E}_{2}$-algebra structure.

We define Hochschild cohomology spectrum $\mathcal{H H}^{\bullet}(\mathcal{C})=\mathcal{H}^{\bullet}(\mathcal{D})$ as $E\left(\mathcal{E}\right.$ nd $\left.(\mathcal{D})^{\otimes}\right) \in \operatorname{Alg}_{\mathbf{E}_{2}}(\operatorname{Sp})$. The underlying associative algebra $\mathcal{H} \mathcal{H}^{\bullet}(\mathcal{C})$ is the endomorphism algebra of the identity functor $\mathcal{D} \rightarrow \mathcal{D}$ in $\operatorname{Fun}^{\mathrm{L}}(\mathcal{D}, \mathcal{D})$.

Consider the counit map of the adjunction:

$$
\operatorname{RMod}_{\mathcal{H} \mathcal{H}^{\bullet}(\mathcal{D})}^{\otimes} \longrightarrow \mathcal{E} \operatorname{nd}(\mathcal{D})^{\otimes}
$$

which is a monoidal functor. Since $\mathcal{E} \operatorname{nd}(\mathcal{D})^{\otimes}$ naturally acts on $\mathcal{D}$, it gives rise to an action of $\operatorname{RMod}_{\mathcal{H} \mathcal{H}^{\bullet}(\mathcal{D})}^{\otimes}$ on $\mathcal{D}$ :

$$
\mathcal{E} \operatorname{nd}(\mathcal{D})^{\otimes} \curvearrowright \mathcal{D} \Rightarrow \operatorname{RMod}_{\mathcal{H} \mathcal{H}^{\bullet}(\mathcal{D})}^{\otimes} \curvearrowright \mathcal{D}
$$

In other words, $\mathcal{D}$ is a left $\operatorname{RMod}_{\mathcal{H} \mathcal{H}^{\bullet}(\mathcal{D})}^{\otimes}$-module object in $\operatorname{Pr}_{\mathrm{St}}^{\mathrm{L}}$. Let $\operatorname{RPerf}_{\mathcal{H} \mathcal{H}^{\bullet}(\mathcal{C})}^{\otimes} \subset \operatorname{RMod}_{\mathcal{H} \mathcal{H}}^{\otimes}(\mathcal{D})$ be the monoidal full subcategory that consists of compact objects. By the restrictions, it gives rise to a left $\operatorname{RPerf}_{\mathcal{H} \mathcal{H} \bullet(\mathcal{C})}^{\otimes}$-module object $\mathcal{C}$ :

$$
\operatorname{RPerf}_{\mathcal{H} \mathcal{H} \bullet(\mathcal{C})}^{\otimes} \curvearrowright \mathcal{C}
$$

in the $\infty$-category $\mathcal{S}$ t of small stable idempotent-complete $\infty$-categories in which morphisms are exact functors ( $\mathcal{S}$ t also admits a suitable symmetric monoidal structure). Informally, we think of it as a categorical associative action of $\mathcal{H H}^{\bullet}(\mathcal{C})$ on $\mathcal{C}$. This is induced by the adjunction so that it has an evident universal property.

Construct a functor $\mathcal{S}$ t $\rightarrow$ Sp which carries $\mathcal{C}$ to the Hochschild homology spectrum $\mathcal{H} \mathcal{H}_{\bullet}(\mathcal{C})$. In the classical differential graded context, Hochschild chain complex comes equipped with the Connes operator. In our general setting, it is natural to encode such structures by means of circle actions: Hochschild homology spectrum $\mathcal{H} \mathcal{H} \bullet(\mathcal{C})$ is promoted to a spectrum with an $S^{1}$-action, 
that is, an object of Fun $\left(B S^{1}, \mathrm{Sp}\right)$. Thus we configure the assignment $\mathcal{C} \mapsto \mathcal{H} \mathcal{H} \bullet(\mathcal{C})$ as a symmetric monoidal functor

$$
\mathcal{H H}_{\bullet}(-): \mathcal{S t}^{\otimes} \longrightarrow \operatorname{Fun}\left(B S^{1}, \mathrm{Sp}\right)^{\otimes},
$$

where $\operatorname{Fun}\left(B S^{1}, \mathrm{Sp}\right)$ inherits a pointwise symmetric monoidal structure from the structure on Sp.

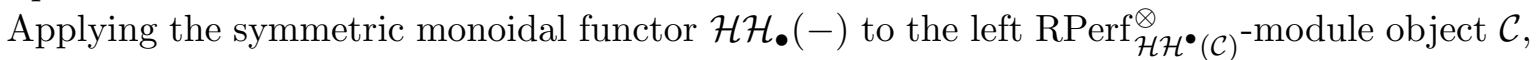
we obtain a left $\mathcal{H}_{\bullet}\left(\operatorname{RPerf}_{\mathcal{H} \mathcal{H}^{\bullet}(\mathcal{C})}\right)$-module $\mathcal{H} \mathcal{H}_{\bullet}(\mathcal{C})$ in $\operatorname{Fun}\left(B S^{1}, \operatorname{Sp}\right)$. Note that $\mathcal{H} \mathcal{H}_{\bullet}\left(\mathcal{H} \mathcal{H}^{\bullet}(\mathcal{C})\right)$ $\simeq \mathcal{H H}_{\bullet}\left(\operatorname{RPerf}_{\mathcal{H} \mathcal{H}^{\bullet}(\mathcal{C})}\right)$ is an associative algebra object in Fun $\left(B S^{1}, \mathrm{Sp}\right)$. In other words, it is an associative ring spectrum equipped with an $S^{1}$-action.

There is a topological operad ( $\infty$-operad) Cyl (defined in a geometric way). We have a canonical equivalence $\operatorname{Alg}_{\mathbf{C y l}}(\mathrm{Sp}) \simeq \operatorname{Alg}_{\mathrm{As}}\left(\operatorname{Fun}\left(B S^{1}, \mathrm{Sp}\right)\right)$ between $\infty$-categories of algebras, where $\operatorname{Alg}_{\mathbf{C y l}}(\mathrm{Sp})$ is the $\infty$-category of algebras over $\mathbf{C y l}$. There is another two-colored topological operad ( $\infty$-operad) DCyl such that operads $\mathbf{E}_{2}^{\otimes}$ and $\mathbf{C y l}$ are contained in DCyl as full suboperads: $\mathbf{E}_{2}^{\otimes} \subset \mathbf{D C y l} \supset \mathbf{C y l}$. Let $i: \mathbf{E}_{2}^{\otimes} \hookrightarrow \mathbf{D C y l}$ denote the inclusion, and let $i_{!}: \operatorname{Alg}_{\mathbf{E}_{2}}(\mathrm{Sp}) \rightarrow$ $\operatorname{Alg}_{\mathbf{D C y l}}(\mathrm{Sp})$ be the left adjoint of the forgetful functor $i^{*}: \operatorname{Alg}_{\mathbf{D C y l}}(\mathrm{Sp}) \rightarrow \operatorname{Alg}_{\mathbf{E}_{2}}(\mathrm{Sp})$. Consider the sequence

$$
\operatorname{Alg}_{\mathbf{E}_{2}}(\mathrm{Sp}) \stackrel{i_{!}}{\longrightarrow} \operatorname{Alg}_{\mathbf{D C y l}}(\mathrm{Sp}) \stackrel{\text { forget }}{\longrightarrow} \operatorname{Alg}_{\mathbf{C y l}}(\mathrm{Sp}) \simeq \operatorname{Alg}_{\mathrm{As}}\left(\operatorname{Fun}\left(B S^{1}, \mathrm{Sp}\right)\right)
$$

and denote by $i_{!}\left(\mathcal{H} \mathcal{H}^{\bullet}(\mathcal{C})\right)_{C}$ the image of $\mathcal{H} \mathcal{H}^{\bullet}(\mathcal{C})$ under the composite. We construct a canonical equivalence $i_{!}\left(\mathcal{H} \mathcal{H}^{\bullet}(\mathcal{C})\right)_{C} \simeq \mathcal{H}_{\bullet}\left(\mathcal{H} \mathcal{H}^{\bullet}(\mathcal{C})\right)$ in $\operatorname{Alg}_{\text {As }}\left(\operatorname{Fun}\left(B S^{1}, \operatorname{Sp}\right)\right)$.

Assembling the constructions, we obtain a triple

$$
\left(\mathcal{H}^{\bullet}(\mathcal{C}), i_{!}\left(\mathcal{H}^{\bullet}(\mathcal{C})\right)_{C} \simeq \mathcal{H}_{\bullet}\left(\mathcal{H H}^{\bullet}(\mathcal{C})\right), \mathcal{H} \mathcal{H}_{\bullet}(\mathcal{C})\right)
$$

where $\mathcal{H H}^{\bullet}(\mathcal{C})$ is the $\mathbf{E}_{2}^{\otimes}$-algebra, $\mathcal{H H}_{\bullet}(\mathcal{C})$ is the left $\mathcal{H}_{\bullet} \cdot\left(\mathcal{H} \mathcal{H}^{\bullet}(\mathcal{C})\right)$-module (in Fun $\left(B S^{1}\right.$, Sp $)$ ). As mentioned in Theorem 1.2, we prove that the triple exactly amounts to a KS-algebra $\left(\mathcal{H} \mathcal{H}^{\bullet}(\mathcal{C}), \mathcal{H} \mathcal{H}_{\bullet}(\mathcal{C})\right)$, that is, the structure of an algebra over the Kontsevich-Soibelman operad $\mathbf{K S}$ on the pair $\left(\mathcal{H H}^{\bullet}(\mathcal{C}), \mathcal{H} \mathcal{H}_{\bullet}(\mathcal{C})\right)$. This completes the construction.

2.2. This paper is organized as follows: Section 3 collects conventions and some of the notation that we will use. In Section 4, we discuss algebras over the Kontsevich-Soibelman operad. The main result of Section 4 is Corollary 4.21 (= Theorem 1.2). Along the way, we introduce several topological colored operads ( $\infty$-operads). In Section 5 , we give a brief review of Hochschild cohomology spectra that we will use. In Section 6, we give a construction of the assignment $\mathcal{C} \mapsto \mathcal{H} \mathcal{H}_{\bullet}(\mathcal{C})$ which satisfies the requirements for our goal (partly because we are not able to find a suitable construction in the literature). The results of this section will be quite useful for various purposes other than the subject of this paper. In Section 7, we prove Theorem 7.14 (= Theorem 1.1). Namely, we construct a KS-algebra $(\mathcal{H} \mathcal{H}(\mathcal{C}), \mathcal{H} \mathcal{H} \bullet(\mathcal{C}))$. In Section 8, we study the action morphisms determined by the structure of the KS-algebra on $\left(\mathcal{H} \mathcal{H}^{\bullet}(\mathcal{C}), \mathcal{H} \mathcal{H}_{\bullet}(\mathcal{C})\right)$. In Section 9 , we give a generalization to an equivariant setting (cf. Theorem 1.3): $\mathcal{C}$ is endowed with the action of a group (a group object in the $\infty$-category of spaces).

\section{Notation and convention}

Throughout this paper we use the theory of quasi-categories. We assume that the reader is familiar with this theory and operads. A quasi-category is a simplicial set which satisfies the weak Kan condition of Boardman-Vogt. The theory of quasi-categories from the viewpoint of models of $(\infty, 1)$-categories were extensively developed by Joyal and Lurie [21, 25, 27]. Following [27], we shall refer to quasi-categories as $\infty$-categories. Our main references are [25] and [27]. Given 
an ordinary category $\mathcal{C}$, by passing to the nerve $\mathrm{N}(\mathcal{C})$, we think of $\mathcal{C}$ as the $\infty$-category $\mathrm{N}(\mathcal{C})$. We usually abuse notation by writing $\mathcal{C}$ for $\mathrm{N}(\mathcal{C})$ even when $\mathcal{C}$ should be thought of as a simplicial set or an $\infty$-category.

We use the theory of $\infty$-operads which is thoroughly developed in [25]. The notion of $\infty$-operads gives one of the models of colored operads. Thanks to Hinich [15], there is a comparison between algebras over differential graded operads and algebras over $\infty$-operads in values in chain complexes. In particular, in characteristic zero, [15] establishes an equivalence between two notions of algebras, see loc. cit.

Here is a list of some of the conventions and notation that we will use:

- $\mathbf{Z}$ : the ring of integers, $\mathbf{R}$ denotes the set of real numbers which we regard as either a topological space or a ring.

- $\Delta$ : the category of linearly ordered non-empty finite sets (consisting of [0], [1], .., $[n]=\{0, \ldots, n\}, \ldots)$.

- $\Delta^{n}$ : the standard $n$-simplex.

- $\mathrm{N}$ : the simplicial nerve functor (cf. [27, Section 1.1.5]).

- $\mathcal{S}$ : $\infty$-category of small spaces. We denote by $\widehat{\mathcal{S}}$ the $\infty$-category of large spaces (cf. [27, Section 1.2.16]).

- $\mathcal{C} \simeq$ : the largest Kan subcomplex of an $\infty$-category $\mathcal{C}$.

- $\mathcal{C}^{\text {op: }}$ the opposite $\infty$-category of an $\infty$-category. We also use the superscript "op" to indicate the opposite category for ordinary categories and enriched categories.

- Cat $_{\infty}$ : the $\infty$-category of small $\infty$-categories.

- Sp: the stable $\infty$-category of spectra.

- $\operatorname{Fun}(A, B)$ : the function complex for simplicial sets $A$ and $B$.

- $\operatorname{Fun}_{C}(A, B)$ : the simplicial subset of $\operatorname{Fun}(A, B)$ classifying maps which are compatible with given projections $A \rightarrow C$ and $B \rightarrow C$.

- $\operatorname{Map}(A, B)$ : the largest $\operatorname{Kan}$ subcomplex of $\operatorname{Fun}(A, B)$ when $A$ and $B$ are $\infty$-categories.

- $\operatorname{Map}_{\mathcal{C}}\left(C, C^{\prime}\right)$ : the mapping space from an object $C \in \mathcal{C}$ to $C^{\prime} \in \mathcal{C}$, where $\mathcal{C}$ is an $\infty$ category. We usually view it as an object in $\mathcal{S}$ (cf. [27, Section 1.2.2]).

- $\operatorname{Fin}_{*}$ : the category of pointed finite sets $\langle 0\rangle,\langle 1\rangle, \ldots\langle n\rangle, \ldots$, where $\langle n\rangle=,\{*, 1, \ldots, n\}$ with the base point $*$. We write $\Gamma$ for $\mathrm{N}\left(\operatorname{Fin}_{*}\right) .\langle n\rangle^{\circ}=\langle n\rangle \backslash *$. Notice that the (nerve of) Segal's gamma category is the opposite category of our $\Gamma$.

- $\mathcal{P}^{\text {act }}$ : If $\mathcal{P}$ is an $\infty$-operad, we write $\mathcal{P}^{\text {act }}$ for the subcategory of $\mathcal{P}$ spanned by active morphisms.

- Triv $^{\otimes}$ : the trivial $\infty$-operad [25, Example 2.1.1.20].

- $\mathrm{As}^{\otimes}$ : the associative operad [25, Section 4.1.1], we use the notation slightly different from loc. cit. Informally, an As-algebra (an algebra over $\mathrm{As}^{\otimes}$ ) is an unital associative algebra. For a symmetric monoidal $\infty$-category $\mathcal{C}^{\otimes}$, we write $\operatorname{Alg}_{\text {As }}(\mathcal{C})$ for the $\infty$-category of As-algebra objects. We refer to an object of $\operatorname{Alg}_{A s}(\mathcal{C})$ as an associative algebra object in $\mathcal{C}^{\otimes}$. We refer to a monoidal $\infty$-category over $\mathrm{As}^{\otimes}$ as an associative monoidal $\infty$-category.

- $\mathcal{L} \mathcal{M}^{\otimes}$ : the $\infty$-operad defined in [25, Definition 4.2.1.7]. An algebra over $\mathcal{L} \mathcal{M}^{\otimes}$ is a pair $(A, M)$ such that an unital associative algebra $A$ and a left $A$-module $M$. For a symmetric monoidal $\infty$-category $\mathcal{C}^{\otimes} \rightarrow \Gamma$, we write $\operatorname{LMod}\left(\mathcal{C}^{\otimes}\right)$ or $\operatorname{LMod}(\mathcal{C})$ for $\operatorname{Alg}_{\mathcal{L M}^{\otimes}}\left(\mathcal{C}^{\otimes}\right)$.

- $\mathbf{E}_{n}^{\otimes}$ : the $\infty$-operad of little $n$-cubes. For a symmetric monoidal $\infty$-category $\mathcal{C}^{\otimes}$, we write $\operatorname{Alg}_{\mathbf{E}_{n}}(\mathcal{C})$ for the $\infty$-category of $\mathbf{E}_{n}$-algebra objects. 


\section{Operads}

4.1. We will define several simplicial colored operads which are relevant to us. By a simplicial colored operad, we mean a colored operad in the symmetric monoidal category of simplicial sets. A simplicial colored operad is also referred to as a symmetric multicategory enriched over the category of simplicial sets.

Definition 4.1. Let $(0,1)$ denote the open interval $\{x \in \mathbf{R} \mid 0<x<1\}$. For $n \geq 0$, let $(0,1)^{n}$ be the $n$-fold product, i.e., the $n$-dimensional cube. An open embedding $f:(0,1)^{n} \rightarrow(0,1)^{n}$ is said to be rectilinear if it is given by

$$
f\left(x_{1}, \ldots, x_{n}\right)=\left(a_{1} x_{1}+b_{1}, \ldots, a_{n} x_{n}+b_{n}\right)
$$

for some real constants $0<a_{1}, \ldots, a_{n} \leq 1,0 \leq b_{1}, \ldots, b_{n}<1$, provided that the formula defines an embedding. An embedding $f:(0,1)^{n} \rightarrow(0,1)^{n}$ is said to be shrinking if it is given by $f\left(x_{1}, \ldots, x_{n}\right)=\left(a_{1} x_{1}, \ldots, a_{n} x_{n}\right)$ for some $0<a_{1}, \ldots, a_{n} \leq 1$.

Let $S^{1}$ denote the circle $\mathbf{R} / \mathbf{Z}$ which we regard as a topological space. A continuous map $f:(0,1)^{n} \times S^{1} \rightarrow(0,1)^{n} \times S^{1}$ is said to be rectilinear (resp. shrinking) if $f=(\phi, \psi)$ such that $\phi:(0,1)^{n} \rightarrow(0,1)^{n}$ is rectilinear (resp. shrinking) and $\psi: S^{1} \rightarrow S^{1}$ is given by a rotation

$$
S^{1}=\mathbf{R} / \mathbf{Z} \ni x \mapsto x+r \in \mathbf{R} / \mathbf{Z}=S^{1}
$$

with $r \in \mathbf{R} / \mathbf{Z}$. In particular, when $n=0, f: S^{1} \rightarrow S^{1}$ is rectilinear if it is given by a rotation.

Let $n \geq 1$. If a continuous map $f:(0,1)^{n} \rightarrow(0,1)^{n-1} \times S^{1}$ is said to be rectilinear if it factors as $(0,1)^{n} \stackrel{g}{\rightarrow}(0,1)^{n-1} \times \mathbf{R} \stackrel{h}{\rightarrow}(0,1)^{n-1} \times \mathbf{R} / \mathbf{Z}$, where $h$ is the projection, and $g$ is given by $f\left(x_{1}, \ldots, x_{n}\right)=\left(a_{1} x_{1}+b_{1}, \ldots, a_{n} x_{n}+b_{n}\right)$ for some real constants $0<a_{1}, \ldots, a_{n} \leq 1$, $0 \leq b_{1}, \ldots, b_{n-1}<1, b_{n} \in \mathbf{R}$, provided that the formula defines an open embedding.

Definition 4.2. Let Cyl be a simplicial colored operad defined as follows:

(i) The set of colors of Cyl has a single element, which we will denote by $C$.

(ii) Let $I=\langle r\rangle^{\circ}$ be a finite set and let $\{C\}_{I}$ be a set of colors indexed by $I$. By abuse of notation, we write $C^{\sqcup r}$ for $\{C\}_{I}$, where $r$ is the number of elements of $I$. We remark that $C^{\sqcup r}$ does not mean the coproduct. We define $\operatorname{Mult}_{\mathrm{Cyl}}\left(\{C\}_{I}, C\right)=\operatorname{Mult}_{\mathrm{Cyl}}\left(C^{\sqcup r}, C\right)$ to be the singular simplicial complex of the space

$$
\operatorname{Emb}^{\mathrm{rec}}\left(\left((0,1) \times S^{1}\right)^{\sqcup r},(0,1) \times S^{1}\right)
$$

of embeddings $\left((0,1) \times S^{1}\right)^{\sqcup r} \rightarrow(0,1) \times S^{1}$ such that the restriction to each component $(0,1) \times S^{1} \rightarrow(0,1) \times S^{1}$ is rectilinear. Here $\left((0,1) \times S^{1}\right)^{\sqcup r}$ is the disjoint union of $(0,1) \times$ $S^{1}$, whose set of connected components is identified with $I$. The space $\operatorname{Emb}^{\text {rec }}(((0,1) \times$ $\left.\left.S^{1}\right)^{\sqcup r},(0,1) \times S^{1}\right)$ is endowed with the standard topology, that is, the subspace of the mapping space with compact-open topology.

(iii) The composition law in $\mathrm{Cyl}$ is given by the composition of rectilinear embeddings, and a unit map is the identity map.

The color $C$ together with $\operatorname{Mult}_{\mathrm{Cyl}}\left(\{C\}_{I}, C\right)$ constitutes a fibrant simplicial colored operad. By a fibrant simplicial colored operad we mean that every simplicial set $\operatorname{Mult}_{C y l}\left(\{C\}_{I}, C\right)$ is a Kan complex. Note that the singular simplicial complex of a topological space is a Kan complex.

Definition 4.3. Let $\overline{\mathrm{Cyl}}$ be a simplicial colored operad defined as follows: 
(i) The set of colors of $\overline{\mathrm{Cyl}}$ has two elements denoted by $C$ and $C_{M}$.

(ii) Let $I=\langle r\rangle^{\circ}$ be a finite set and let $\left\{C, C_{M}\right\}_{I}$ be a set of colors indexed by $I$, which we think of as a map $p: I \rightarrow\left\{C, C_{M}\right\}$. We also write $C^{\sqcup m} \sqcup C_{M}^{\sqcup n}$ for $\left\{C, C_{M}\right\}_{I}$ when $p^{-1}(C)$ (resp. $\left.p^{-1}\left(C_{M}\right)\right)$ has $m$ elements (resp. $n$ elements). Let

$$
\operatorname{Emb}^{\mathrm{rec}}\left(\left((0,1) \times S^{1}\right)^{\sqcup m} \sqcup\left((0,1) \times S^{1}\right)^{\sqcup n},(0,1) \times S^{1}\right)
$$

denote the space of embeddings $\left((0,1) \times S^{1}\right)^{\sqcup m} \sqcup\left((0,1) \times S^{1}\right)^{\sqcup n} \rightarrow(0,1) \times S^{1}$ such that the restriction to each component is rectilinear (the topology is induced by compact-open topology). We refer to it as the space of rectilinear embeddings. For $n \geq 1$, let

$$
\begin{aligned}
& \text { Mult } \frac{t}{\mathrm{Cyl}}\left(C^{\sqcup m} \sqcup C_{M}^{\sqcup n}, C_{M}\right) \\
& \quad:=\mathrm{Emb}^{\mathrm{rec}}\left(\left((0,1) \times S^{1}\right) \times p^{-1}(C) \sqcup\left((0,1) \times S^{1}\right) \times p^{-1}\left(C_{M}\right),(0,1) \times S^{1}\right)
\end{aligned}
$$

be its subspace that consists of those rectilinear embeddings $f$ such that each restriction to any component in $\left((0,1) \times S^{1}\right) \times p^{-1}\left(C_{M}\right)$ is (not only rectilinear but also) shrinking. Here $\left((0,1) \times S^{1}\right) \times p^{-1}(C) \sqcup\left((0,1) \times S^{1}\right) \times p^{-1}\left(C_{M}\right)$ denotes the finite disjoint union of $(0,1) \times S^{1}$ indexed by $p^{-1}(C) \sqcup p^{-1}\left(C_{M}\right) \simeq I$, but we distinguish between components indexed by $p^{-1}(C)$ and those indexed in $p^{-1}\left(C_{M}\right)$ since they play different roles. Notice that Mult $\frac{t}{\mathrm{Cyl}}\left(C^{\sqcup m} \sqcup C_{M}^{\sqcup n}, C_{M}\right)$ is the empty space for $n \geq 2$. We define Mult $\overline{\mathrm{Cyl}}\left(C^{\sqcup m} \sqcup\right.$ $\left.C_{M}^{\sqcup n}, C_{M}\right)$ to be the singular simplicial complex of Mult $\frac{t}{\mathrm{Cyl}}\left(C^{\sqcup m} \sqcup C_{M}^{\sqcup n}, C_{M}\right)$. When $n=0$, we define Mult $\overline{\mathrm{Cyl}}\left(C^{\sqcup m}, C_{M}\right)$ to be the empty simplicial set.

(iii) We set Mult $\overline{\mathrm{Cyl}}\left(C^{\sqcup m}, C\right)=\operatorname{Mult}_{\mathrm{Cyl}}\left(C^{\sqcup m}, C\right)$. If $n \neq 0, \operatorname{Mult}_{\overline{\mathrm{Cyl}}}\left(C^{\sqcup m} \sqcup C_{M}^{\sqcup n}, C\right)$ is the empty set.

(iv) The composition law is given by the composition of rectilinear embeddings, and a unit map is the identity map.

The colors $C, C_{M}$ together with simplicial sets of maps constitute a fibrant simplicial colored operad.

Definition 4.4. Let DCyl be a simplicial colored operad defined as follows:

(i) The set of colors of DCyl has two elements, which we denoted by $D$ and $C$.

(ii) Let $I=\langle r\rangle^{\circ}$ be a finite set and let $\{D, C\}_{I}$ be a set of colors indexed by $I$, that is, a map $p: I \rightarrow\{D, C\}$. By abuse of notation we write $D^{\sqcup l} \sqcup C^{\sqcup m}$ for $\{D, C\}_{I}$ when $p^{-1}(D)$ (resp. $p^{-1}(C)$ ) has $l$ elements (resp. $m$ elements). We define $\operatorname{Mult}_{\mathrm{DCyl}}\left(D^{\sqcup l}, D\right)$ to be the singular simplicial complex of the space

$$
\operatorname{Emb}^{\mathrm{rec}}\left(\left((0,1)^{2}\right)^{\sqcup l},(0,1)^{2}\right)
$$

of embeddings from the disjoint union $(0,1)^{2} \times p^{-1}(D)$ to $(0,1)^{2}$ such that the restriction to each component is rectilinear, where the space comes equipped with the subspace topology of the mapping space with compact-open topology.

If $m \geq 1$, Mult $\overline{\mathrm{Cyl}}\left(D^{\sqcup l} \sqcup C^{\sqcup m}, D\right)$ is the empty set.

(iii) We define Mult $\operatorname{DCyl}\left(D^{\sqcup l} \sqcup C^{\sqcup m}, C\right)$ to be the singular complex of the space of embeddings

$$
\operatorname{Emb}^{\text {rec }}\left((0,1)^{2} \times p^{-1}(D) \sqcup\left((0,1) \times S^{1}\right) \times p^{-1}(C),(0,1) \times S^{1}\right)
$$

such that the rescriction to a component $(0,1)^{2}$ is rectilinear, and the restriction to a component $(0,1) \times S^{1}$ is rectilinear. 
(iv) The composition law and the unit are defined in an obvious way.

The colors $D, C$ together with simplicial sets of maps constitute a fibrant simplicial colored operad.

Definition 4.5. Let $\overline{\mathrm{DCyl}}$ be a simplicial colored operad defined as follows.

(i) The set of colors of $\overline{\mathrm{DCyl}}$ has three elements, which we denote by $D, C$, and $C_{M}$.

(ii) Let $I=\langle r\rangle^{\circ}$ be a finite set and let $\left\{D, C, C_{M}\right\}_{I}$ be a set of colors indexed by $I$, that is, a map $p: I \rightarrow\left\{D, C, C_{M}\right\}$. By abuse of notation, we write $D^{\sqcup l} \sqcup C^{\sqcup m} \sqcup C_{M}^{\sqcup n}$ for $\left\{D, C, C_{M}\right\}_{I}$ when $p^{-1}(D)$ (resp. $p^{-1}(C), p^{-1}\left(C_{M}\right)$ ) has $l$ elements (resp. $m$ elements, $n$ elements). We set Mult $\overline{\mathrm{DCyl}}\left(D^{\sqcup l}, D\right)=\operatorname{Mult}_{\mathrm{DCyl}}\left(D^{\sqcup l}, D\right)$. If $m+n \geq 1$, Mult $\overline{\mathrm{Cyl}}\left(D^{\sqcup l} \sqcup\right.$ $\left.C^{\sqcup m} \sqcup C_{M}^{\sqcup n}, D\right)$ is the empty set.

(iii) We set Mult $\overline{\overline{D C y l}}\left(D^{\sqcup l} \sqcup C^{\sqcup m}, C\right)=\operatorname{Mult}_{\mathrm{DCyl}}\left(D^{\sqcup l} \sqcup C^{\sqcup m}, C\right)$. If $n \geq 1$,

$$
\operatorname{Mult}_{\overline{\mathrm{DCyl}}}\left(D^{\sqcup l} \sqcup C^{\sqcup m} \sqcup C_{M}^{\sqcup n}, C\right)
$$

is the empty set.

(iv) For $n \neq 1$, Mult $\overline{\mathrm{DCyl}}\left(D^{\sqcup l} \sqcup C^{\sqcup m} \sqcup C_{M}^{\sqcup n}, C_{M}\right)$ is the empty set. For $n=1$,

$$
\operatorname{Mult}_{\overline{\mathrm{DCyl}}}\left(D^{\sqcup l} \sqcup C^{\sqcup m} \sqcup C_{M}, C_{M}\right)
$$

is the singular simplicial complex of the space of embeddings

$$
(0,1)^{2} \times p^{-1}(D) \sqcup\left((0,1) \times S^{1}\right) \times p^{-1}(C) \sqcup\left((0,1) \times S^{1}\right) \times p^{-1}\left(C_{M}\right) \rightarrow(0,1) \times S^{1}
$$

such that the restriction to a component in $(0,1)^{2} \times p^{-1}(D)$ is rectilinear, the restriction to each component in $\left((0,1) \times S^{1}\right) \times p^{-1}(C)$ is rectilinear, and the restriction to $((0,1) \times$ $\left.S^{1}\right) \times p^{-1}\left(C_{M}\right) \simeq(0,1) \times S^{1}$ is shrinking. By definition, if $l=0$, Mult $\overline{\overline{\mathrm{DCyl}}}\left(C^{\sqcup m} \sqcup\right.$ $\left.C_{M}, C_{M}\right)=$ Mult $_{\overline{\mathrm{Cyl}}}\left(C^{\sqcup m} \sqcup C_{M}, C_{M}\right)$.

(v) The composition law and the unit map are defined in an obvious way.

The colors $D, C, C_{M}$ together with simplicial sets of maps constitute a fibrant simplicial colored operad.

Remark 4.6. There is a commutative diagram of inclusions of simplicial colored operads:

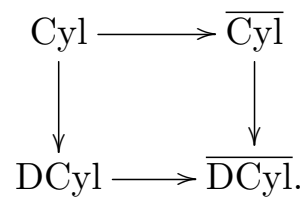

Each inclusion determines a simplicial colored full suboperad.

We obtain an $\infty$-operad from a fibrant simplicial colored operad. We recall the construction from [25, Notation 2.1.1.22].

Definition 4.7. Let $P$ be a simplicial colored operad. Let $P_{\text {col }}$ be the set of colors of $P$. We let $P_{\Delta}$ be a simplicial category defined as follows:

(i) The objects of $P_{\Delta}$ are maps $a:\langle n\rangle^{\circ} \rightarrow P_{\text {col }}$, that is, pairs $\left(\langle n\rangle,\left(C_{1}, \ldots, C_{n}\right)\right)$, where $\langle n\rangle \in \operatorname{Fin}_{*}$ and $\left(C_{1}, \ldots, C_{n}\right)$ is a finite sequence $(a(1), \ldots, a(n))$ of colors. 
(ii) Let $C=\left(\langle n\rangle,\left(C_{1}, \ldots, C_{n}\right)\right)$ and $C^{\prime}=\left(\langle m\rangle,\left(C_{1}^{\prime}, \ldots, C_{m}^{\prime}\right)\right)$ be two objects. The hom simplicial set $\operatorname{Map}_{P_{\Delta}}\left(C, C^{\prime}\right)$ is given by

$$
\coprod_{\alpha:\langle n\rangle \rightarrow\langle m\rangle} \prod_{j \in\langle m\rangle^{\circ}} \operatorname{Mult}_{P}\left(\left\{C_{i}\right\}_{i \in \alpha^{-1}(j)}, C_{j}^{\prime}\right) .
$$

(iii) Composition is determined by the composition laws on Fin $_{*}$ and on $P$ in an obvious way.

There is a canonical simplicial functor $P_{\Delta} \rightarrow$ Fin $_{*}$ which sends $\left(\langle n\rangle,\left(C_{1}, \ldots, C_{n}\right)\right)$ to $\langle n\rangle$. If $P$ is fibrant, the map of simplicial nerves $\mathbf{P}:=\mathrm{N}\left(P_{\Delta}\right) \rightarrow \mathrm{N}\left(\operatorname{Fin}_{*}\right)=\Gamma$ constitutes an $\infty$-operad (cf. [25, Proposition 2.1.1.27]). We shall refer to $\mathrm{N}\left(P_{\Delta}\right) \rightarrow \mathrm{N}\left(\operatorname{Fin}_{*}\right)=\Gamma\left(\right.$ or $\mathrm{N}\left(P_{\Delta}\right)$ ) as the operadic nerve of $P$. We shall denote by $\mathrm{P}_{\langle n\rangle}$ the fiber $\mathbf{P} \times_{\Gamma}\{\langle n\rangle\}$ over $\langle n\rangle$. We usually identify colors with objects in $\mathbf{P}_{\langle 1\rangle}$.

Definition 4.8. We apply the construction in Definiton 4.7 to Cyl, $\overline{\mathrm{Cyl}}, \mathrm{DCyl}$, and $\overline{\mathrm{DCyl}}$ to obtain $\infty$-operads.

- Let Cyl be the operadic nerve of Cyl.

- Let $\overline{\mathbf{C y l}}$ be the operadic nerve of $\overline{\mathrm{Cyl}}$.

- Let DCyl be the operadic nerve of DCyl.

- Let $\overline{\mathbf{D C y l}}$ be the operadic nerve of $\overline{\mathrm{DCyl}}$.

We now recall Kontsevich-Soibelman operad [23].

Definition 4.9. Let KS be the simplicial colored full suboperad of $\overline{\mathrm{DCyl}}$ which consists of colors $D, C_{M}$. We refer to $\mathrm{KS}$ as Kontsevich-Soibelman operad. Let $\mathbf{K S}$ be the operadic nerve of KS (the notation is slightly diffrent from Introduction). We abuse terminology by referring to it as Kontsevich-Soibelman operad. In a nutshell, KS $\subset \overline{\mathbf{D C y l}}$ is the maximal simplicial subcomplex spanned by vertices correponding to those tuples which do not contain the color $C$. It is not difficult to check that $\mathrm{KS}$ is equivalent to that of [16] or [23, Section 11.2]. In [11], a version of $\mathrm{KS}$ is called the cylinder operad.

Remark 4.10. We note that DCyl has a simplicial full suboperad $\langle D\rangle \subset$ DCyl which consists of the single color $D$. This operad $\langle D\rangle$ is a version of the little 2-cube operad (e.g., [25, Definition 5.1.0.1]). Let $\mathbf{E}_{2}^{\otimes}$ be the operadic nerve of $\langle D\rangle$, which we shall refer to as the $\infty$-operad of little 2-cubes.

Remark 4.11. We have the diagram in Remark 4.6 and inlusions $\mathrm{KS} \subset \overline{\mathrm{DCyl}},\langle D\rangle \subset \mathrm{DCyl}$. These inclusions determine the following diagram of $\infty$-operads:

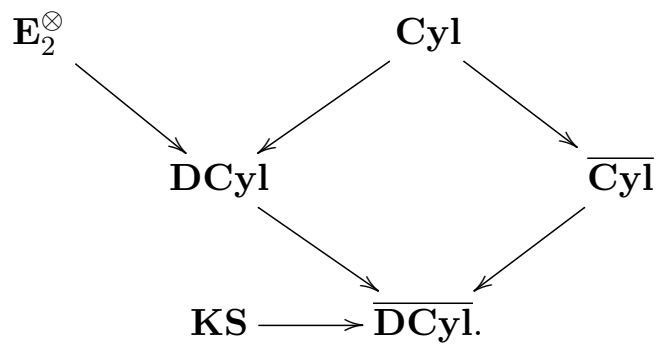

Let $\mathrm{E}_{1}$ be the simplicial operad of little 1-cubes. The definition is similar to the case of little 2-cubes (see, e.g., [25, Proposition 5.1.0.3]). Namely, $\mathrm{E}_{1}$ has a single color $D_{1}$, and for a finite sequence $\left(D_{1}, \ldots, D_{1}\right)$, the simplicial set $\operatorname{Mult}_{\mathrm{E}_{1}}\left(D_{1}^{\sqcup n}, D_{1}\right)$ is defined to be the singular simplicial complex of the space $\operatorname{Emb}^{\text {rec }}\left((0,1)^{\sqcup n},(0,1)\right)$ of rectilinear embeddings. The composition law and the unit are defined in the obvious way. Let $\mathbf{E}_{1}^{\otimes}$ denote the $\infty$-operad of little 1-cubes, that is, the operadic nerve of $\mathrm{E}_{1}$. 
Definition 4.12. Let $\overline{\mathrm{E}}_{1}$ be a simplicial colored operad defined as follows.

(i) The set of colors of $\overline{\mathrm{E}}_{1}$ has two elements which we denote by $D_{1}$ and $M$.

(ii) Let $I=\langle r\rangle^{\circ}$ be a finite set and let $\left\{D_{1}, M\right\}_{I}$ be a set of colors indexed by $I$, which is a map $p: I \rightarrow\left\{D_{1}, M\right\}$. We write $D_{1}^{\sqcup m} \sqcup M^{\sqcup n}$ for $\left\{D_{1}, M\right\}_{J}$ when $p^{-1}\left(D_{1}\right)\left(\operatorname{resp} . p^{-1}(M)\right)$ has $m$ elements (resp. $n$ elements). Let $\operatorname{Emb}^{\text {rec }}\left((0,1)^{\sqcup m} \sqcup(0,1),(0,1)\right)$ be the topological space of embeddings $(0,1)^{\sqcup m} \sqcup(0,1) \rightarrow(0,1)$ such that the restriction to each component is rectilinear. We define $\operatorname{Mult}_{\overline{\mathrm{E}}_{1}}\left(D_{1}^{\sqcup m} \sqcup M, M\right)$ to be the singular simplicial complex of the subspace

$$
\operatorname{Emb}^{\mathrm{rec}}\left((0,1) \times p^{-1}\left(D_{1}\right) \sqcup(0,1) \times p^{-1}(M),(0,1)\right) \subset \operatorname{Emb}^{\mathrm{rec}}\left((0,1)^{\sqcup m} \sqcup(0,1),(0,1)\right) .
$$

The subspace consists of those rectilinear embeddings such that the restriction to $(0,1) \times$ $p^{-1}(M) \simeq(0,1)$ is shrinking. If $n \neq 1$, Mult $_{\overline{\mathrm{E}}_{1}}\left(D_{1}^{\sqcup m} \sqcup M^{\sqcup n}, M\right)$ is the empty set.

(iii) We set $\operatorname{Mult}_{\overline{\mathrm{E}}_{1}}\left(D_{1}^{\sqcup n}, D_{1}\right)=\operatorname{Mult}_{\mathrm{E}_{1}}\left(D_{1}^{\sqcup n}, D_{1}\right)$. If $m \neq 0$, $\operatorname{Mult}_{\overline{\mathrm{E}}_{1}}\left(D_{1}^{\sqcup n} \sqcup M^{\sqcup m}, D_{1}\right)$ is the empty set.

(iv) The composition law is given by the composition of embeddings, and a unit map is the identity map.

Let $\overline{\mathbf{E}}_{1}^{\otimes}$ be the operadic nerve of $\overline{\mathrm{E}}_{1}$.

Remark 4.13. There is an equivalence from the $\infty$-operad $\mathbf{E}_{1}^{\otimes}$ to the associative $\infty$-operad Assoc $^{\otimes}$, see [25, Definition 4.1.1.3]. Indeed, if $f:(0,1)^{\sqcup n} \rightarrow(0,1)$ is a rectilinear map, then it determines a linear ordering on the set of connected component $\pi_{0}\left((0,1)^{\sqcup n}\right)$ such that $I_{1}>I_{2}$ for two components $I_{1}$ and $I_{2}$ in $(0,1)^{\sqcup n}$ if $a<b$ for any $a \in f\left(I_{1}\right)$ and any $b \in f\left(I_{2}\right)$. It gives rise to a map from $\operatorname{Emb}^{\mathrm{rec}}\left((0,1)^{\sqcup n},(0,1)\right)$ to the set of linear ordering on $\pi_{0}\left((0,1)^{\sqcup n}\right)$. It is a homotopy equivalence so that we have an equivalence $\mathbf{E}_{1}^{\otimes} \rightarrow \operatorname{Assoc}^{\otimes}$ (for details, see [25, Example 5.1.0.7]).

This equivalence $\mathbf{E}_{1}^{\otimes} \stackrel{\sim}{\rightarrow}$ Assoc $^{\otimes}$ is extended to an equivalence $\overline{\mathbf{E}}_{1}^{\otimes} \stackrel{\sim}{\rightarrow} \mathcal{L} \mathcal{M}^{\otimes}$ of $\infty$-operads, where $\mathcal{L M}^{\otimes}$ is the $\infty$-operad (having two colors) which we use to describe pairs of associative algebras and left modules [25, Definition 4.2.1.7]. Indeed, as above, any map $(0,1)^{\sqcup n} \sqcup(0,1) \rightarrow$ $(0,1)$ in (ii) Definition 4.12 determines a linear ordering on $\pi_{0}\left((0,1)^{\sqcup n} \sqcup(0,1)\right)$ such that the black component is the maximal element. It is easy to see that this ordering induces an equivalence $\overline{\mathbf{E}}_{1}^{\otimes} \rightarrow \mathcal{L} \mathcal{M}^{\otimes}$ which extends $\mathbf{E}_{1}^{\otimes} \stackrel{\sim}{\rightarrow}$ Assoc $^{\otimes}$.

4.2. Following [25], we recall the notion of algebras over an $\infty$-operad. Let $\mathcal{O} \rightarrow \Gamma$ be an $\infty$ operads. Let $\mathcal{M}^{\otimes} \rightarrow \Gamma$ be a symmetric monoidal $\infty$-category whose underlying $\infty$-category is $\mathcal{M}=\mathcal{M}_{\langle 1\rangle}$. An $\mathcal{O}$-algebra in $\mathcal{M}$ is a map $f: \mathcal{O} \rightarrow \mathcal{M}^{\otimes}$ over $\Gamma$ which preserve inert morphisms, that is, a map of $\infty$-operads. We define $\operatorname{Alg}_{\mathcal{O}}\left(\mathcal{M}^{\otimes}\right) \subset \operatorname{Fun}_{\Gamma}\left(\mathcal{O}, \mathcal{M}^{\otimes}\right)$ to be the full subcategory of $\operatorname{Fun}_{\Gamma}\left(\mathcal{O}, \mathcal{M}^{\otimes}\right)$ spanned by $\mathcal{O}$-algebras. We often write $\operatorname{Alg}_{\mathcal{O}}(\mathcal{M})$ for $\operatorname{Alg}_{\mathcal{O}}\left(\mathcal{M}^{\otimes}\right)$ when the structure on $\mathcal{M}$ is clear. We refer to $\operatorname{Alg}_{\mathcal{O}}\left(\mathcal{M}^{\otimes}\right)$ as the $\infty$-category of $\mathcal{O}$-algebra objects in $\mathcal{M}$, cf. [25, Definition 2.1.3.1]. When $\mathcal{O}$ is $\Gamma$, we write $\operatorname{CAlg}\left(\mathcal{M}^{\otimes}\right)$ for $\operatorname{Alg}_{\Gamma}\left(\mathcal{M}^{\otimes}\right)$.

Let $\mathbf{E}_{1}^{\otimes} \rightarrow \Gamma$ be the $\infty$-operad of little 1-cubes with the natural projection. Let $\left(B S^{1}\right)_{\Delta}$ be the simplicial category having a single object $*$ and Hom simplicial set $\operatorname{Hom}_{\left(B S^{1}\right)_{\Delta}}(*, *)$. The simplicial set $\operatorname{Hom}_{\left(B S^{1}\right)_{\Delta}}(*, *)$ is the simplicial complex of $S^{1}=\mathbf{R} / \mathbf{Z}$, and the composition is induced by the ordinary multiplication $S^{1} \times S^{1} \rightarrow S^{1}$. We denote by $B S^{1}$ the simplicial nerve of $\left(B S^{1}\right)_{\Delta}$. It can also be regarded as the classifying space of $S^{1}$ in $\mathcal{S}$. Let $p: \mathbf{E}_{1}^{\otimes} \times B S^{1} \rightarrow \Gamma$ be the composite $\mathbf{E}_{1}^{\otimes} \times B S^{1} \stackrel{\mathrm{pr}_{1}}{\rightarrow} \mathbf{E}_{1}^{\otimes} \rightarrow \Gamma$. We note that $\mathbf{E}_{1}^{\otimes} \times B S^{1} \rightarrow \Gamma$ is not an $\infty$-operad. Let $\mathcal{M}^{\otimes} \rightarrow \Gamma$ be a symmetric monoidal $\infty$-category. Though the above definition of algebra objects is not applicable to $\mathbf{E}_{1}^{\otimes} \times B S^{1} \rightarrow \Gamma$, we define $\operatorname{Alg}_{\mathbf{E}_{1}^{\otimes} \times B S^{1}}\left(\mathcal{M}^{\otimes}\right)$ as follows (cf. [25, Definition 2.3.3.20]). Let $\rho_{i}:\langle n\rangle \rightarrow\langle 1\rangle$ be the unique inert morphism which sends $i \in\langle n\rangle$ to $1 \in\langle 1\rangle$. Then $\operatorname{Alg}_{\mathbf{E}_{1}^{\otimes} \times B S^{1}}\left(\mathcal{M}^{\otimes}\right)$ is the full subcategory of $\operatorname{Fun}_{\Gamma}\left(\mathbf{E}_{1}^{\otimes} \times B S^{1}, \mathcal{M}^{\otimes}\right)$ spanned 
by those maps $F: \mathbf{E}_{1}^{\otimes} \times B S^{1} \rightarrow \mathcal{M}^{\otimes}$ satisfying the condition: If $C$ is an object of $\mathbf{E}_{1}^{\otimes} \times B S^{1}$ lying over $\langle n\rangle$, and for $1 \leq i \leq n \alpha_{i}: C \rightarrow C_{i}$ is a locally $p$-coCartesian morphism covering $\rho_{i}:\langle n\rangle \rightarrow$ $\langle 1\rangle$, then $F\left(\alpha_{i}\right)$ is an inert morphism in $\mathcal{M}^{\otimes}$. Let $\Delta^{0} \rightarrow B S^{1}$ be the natural functor which induces $\mathbf{E}_{1}^{\otimes} \rightarrow \mathbf{E}_{1}^{\otimes} \times B S^{1}$. By the construction of $\mathbf{E}_{1}^{\otimes} \times B S^{1}, F$ belongs to $\operatorname{Alg}_{\mathbf{E}_{1}^{\otimes} \times B S^{1}}\left(\mathcal{M}^{\otimes}\right)$ if and only if the composite $\mathbf{E}_{1}^{\otimes} \rightarrow \mathbf{E}_{1}^{\otimes} \times B S^{1} \stackrel{F}{\rightarrow} \mathcal{M}^{\otimes}$ carries any inert morphism in $\mathbf{E}_{1}^{\otimes}$ lying over $\langle n\rangle \rightarrow\langle 1\rangle$ to an inert morphism in $\mathcal{M}^{\otimes}$. As observed in [25, Remark 2.1.2.9], it is equivalent to the condition that $\mathbf{E}_{1}^{\otimes} \rightarrow \mathbf{E}_{1}^{\otimes} \times B S^{1} \stackrel{F}{\rightarrow} \mathcal{M}^{\otimes}$ carries any inert morphism in $\mathbf{E}_{1}^{\otimes}$ to an inert morphism in $\mathcal{M}^{\otimes}$.

Lemma 4.14. Let $\mathcal{M}^{\otimes}$ be a symmetric monoidal $\infty$-category whose underlying category we denote by $\mathcal{M}$. Let $\operatorname{Fun}\left(B S^{1}, \mathcal{M}\right)$ denote the functor category (function complex) which is endowed with the pointwise symmetric monoidal structure induced by that of $\mathcal{M}^{\otimes}$. Namely, the symmetric monoidal structure on Fun $\left(B S^{1}, \mathcal{M}\right)$ is given by the projection $\operatorname{Fun}\left(B S^{1}, \mathcal{M}\right)^{\otimes}:=$ Fun $\left(B S^{1}, \mathcal{M}^{\otimes}\right) \times_{\text {Fun }\left(B S^{1}, \Gamma\right)} \Gamma \rightarrow \Gamma$. Then there is a canonical equivalence of $\infty$-categories

$$
\operatorname{Alg}_{\mathbf{E}_{1}^{\otimes} \times B S^{1}}\left(\mathcal{M}^{\otimes}\right) \simeq \operatorname{Alg}_{\mathbf{E}_{1}}\left(\operatorname{Fun}\left(B S^{1}, \mathcal{M}\right)^{\otimes}\right) .
$$

Similarly, there is a canonical equivalence $\operatorname{Alg}_{\overline{\mathbf{E}}_{1} \times B S^{1}}\left(\mathcal{M}^{\otimes}\right) \simeq \operatorname{Alg}_{\overline{\mathbf{E}}_{1}}\left(\operatorname{Fun}\left(B S^{1}, \mathcal{M}\right)^{\otimes}\right)$.

Proof. We prove that there is an isomorphism of simplicial sets

$$
\operatorname{Alg}_{\mathbf{E}_{1}^{\otimes} \times B S^{1}}\left(\mathcal{M}^{\otimes}\right) \simeq \operatorname{Alg}_{\mathbf{E}_{1}}\left(\operatorname{Fun}\left(B S^{1}, \mathcal{M}\right)\right) .
$$

Observe that the symmetric monoidal $\infty$-category $\operatorname{Fun}\left(B S^{1}, \mathcal{M}\right)^{\otimes}$ is defined by the following universal property: for a simplicial set $K$, there is a natural bijection of

$$
\operatorname{Hom}_{\text {Set }}\left(K, \operatorname{Fun}\left(B S^{1}, \mathcal{M}\right)^{\otimes}\right)
$$

with the set of pairs $(\alpha, \beta)$ which makes the diagram commute

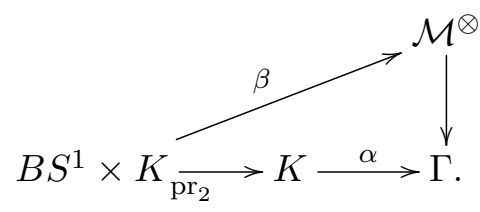

The assignment $(\alpha, \beta) \mapsto \alpha$ induces $\operatorname{Fun}\left(B S^{1}, \mathcal{M}\right)^{\otimes} \rightarrow \Gamma$. Therefore, for a simplicial set $L$, a map $L \rightarrow \operatorname{Alg}_{\mathbf{E}_{1}}\left(\operatorname{Fun}\left(B S^{1}, \mathcal{M}\right)^{\otimes}\right)$ amounts to a map $f: B S^{1} \times L \times \mathbf{E}_{1}^{\otimes} \rightarrow \mathcal{M}^{\otimes}$ over $\Gamma$ such that for any vertex $(a, l)$ in $B S^{1} \times L$ and for any inert morphism $i$ in $\mathbf{E}_{1}^{\otimes}$, the image $f((a, l, i))$ is an inert morphism in $\mathcal{M}^{\otimes}$ (note also that by construction $B S^{1}$ has a single vertex). Next, we consider the universal property of $\operatorname{Alg}_{\mathbf{E}_{1}^{\otimes} \times B S^{1}}\left(\mathcal{M}^{\otimes}\right)$. By the observation before this lemma, for a simplicial set $L$, a map $L \rightarrow \operatorname{Alg}_{\mathbf{E}_{1}^{\otimes} \times B S^{1}}\left(\mathcal{M}^{\otimes}\right)$ amounts to a map $g$ such that the diagram

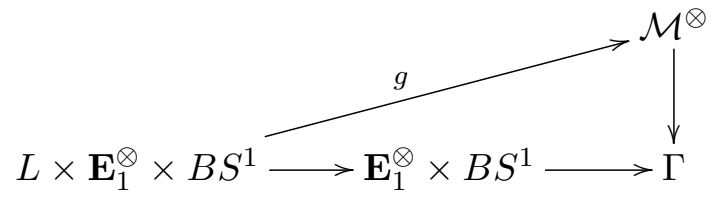

commutes and for any vertex $(l, a)$ in $L \times B S^{1}$ and for any inert morphism $i$ in $\mathbf{E}_{1}^{\otimes}$, the image $g((l, i, a))$ is an inert morphism in $\mathcal{M}^{\otimes}$. Comparing universal properties of $\operatorname{Alg}_{\mathbf{E}_{1}}\left(\operatorname{Fun}\left(B S^{1}, \mathcal{M}\right)\right)$ and $\operatorname{Alg}_{\mathbf{E}_{1}^{\otimes} \times B S^{1}}\left(\mathcal{M}^{\otimes}\right)$, we have a canonical isomorphism of simplicial sets $\operatorname{Alg}_{\mathbf{E}_{1}}\left(\operatorname{Fun}\left(B S^{1}, \mathcal{M}\right)\right)$ $\simeq \operatorname{Alg}_{\mathbf{E}_{1}^{\otimes} \times B S^{1}}\left(\mathcal{M}^{\otimes}\right)$. The final assertion also follows from an argument similar to this proof. 
Construction 4.15. We will define a functor $\mathbf{E}_{1}^{\otimes} \times B S^{1} \rightarrow \mathbf{C y l}$ over $\Gamma$. To this end, we consider the following simplicial categories $\left(\mathrm{E}_{1} \times B S^{1}\right)_{\Delta}$ and $\mathrm{Cyl}_{\Delta}$. Let $\left(\mathrm{E}_{1} \times B S^{1}\right)_{\Delta}$ be a simplicial category defined as follows.

- The objects of $\left(\mathrm{E}_{1} \times B S^{1}\right)_{\Delta}$ are objects $\langle n\rangle$ in $\mathrm{Fin}_{*}$ (which we regard as formal symbols).

- For $\langle n\rangle,\langle m\rangle \in\left(\mathrm{E}_{1} \times B S^{1}\right)_{\Delta}$, the Hom simplicial set $\operatorname{Hom}_{\left(\mathrm{E}_{1} \times B S^{1}\right)_{\Delta}}(\langle n\rangle,\langle m\rangle)$ is the singular complex of the space

$$
\coprod_{\alpha:\langle n\rangle \rightarrow\langle m\rangle}\left(\prod_{1 \leq j \leq m} \operatorname{Emb}^{\mathrm{rec}}\left((0,1) \times \alpha^{-1}(j),(0,1)\right)\right) \times S^{1} .
$$

- The composition is determined by the composition of embeddings and the multiplication $S^{1} \times S^{1} \rightarrow S^{1}$.

There is a canoncial projection $\left(\mathrm{E}_{1} \times B S^{1}\right)_{\Delta} \rightarrow \mathrm{Fin}_{*}$. The simplicial nerve $\mathrm{N}\left(\left(\mathrm{E}_{1} \times B S^{1}\right)_{\Delta}\right) \rightarrow$ $\mathrm{N}\left(\operatorname{Fin}_{*}\right)=\Gamma$ is $p: \mathbf{E}_{1}^{\otimes} \times B S^{1} \rightarrow \Gamma$.

- The objects of $\mathrm{Cyl}_{\Delta}$ are objects $\langle n\rangle$ in $\mathrm{Fin}_{*}$ (which we regard as formal symbols).

- For $\langle n\rangle,\langle m\rangle \in \mathrm{Cyl}_{\Delta}$, the Hom simplicial set $\operatorname{Hom}_{\mathrm{Cyl}_{\Delta}}(\langle n\rangle,\langle m\rangle)$ is the singular simplicial complex of the space

$$
\coprod_{\alpha:\langle n\rangle \rightarrow\langle m\rangle} \prod_{1 \leq j \leq m} \operatorname{Emb}^{\mathrm{rec}}\left((0,1) \times S^{1} \times \alpha^{-1}(j),(0,1) \times S^{1}\right) .
$$

The composition is determined by the composition of embeddings.

Passing to simplicial nerves, we get $\mathrm{N}\left(\mathrm{Cyl}_{\Delta}\right) \simeq \mathbf{C y l} \rightarrow \Gamma$. Note that there is a canonical homeomorphism of spaces

$$
\operatorname{Emb}^{\mathrm{rec}}\left((0,1) \times S^{1} \times \alpha^{-1}(j),(0,1) \times S^{1}\right) \simeq \operatorname{Emb}^{\mathrm{rec}}\left((0,1) \times \alpha^{-1}(j),(0,1)\right) \times\left(S^{1}\right)^{\times \sharp \alpha^{-1}(j)}
$$

(each factor $a \in\left(S^{1}\right)^{\times \sharp \alpha^{-1}(j)}$ determines the rotation of the restriction $\left.(0,1) \times S^{1} \rightarrow(0,1) \times S^{1}\right)$. The diagonal map $S^{1} \rightarrow\left(S^{1}\right)^{\times \sharp \alpha^{-1}(j)}$ induces a map

$$
\operatorname{Hom}_{\left(\mathrm{E}_{1} \times B S^{1}\right)_{\Delta}}(\langle n\rangle,\langle m\rangle) \rightarrow \operatorname{Hom}_{\mathrm{Cyl}_{\Delta}}(\langle n\rangle,\langle m\rangle)
$$

in the natural way. It gives rise to a functor $Z:\left(\mathrm{E}_{1} \times B S^{1}\right)_{\Delta} \rightarrow \mathrm{Cyl}_{\Delta}$ of simplicial categories. Then it induces a map

$$
z: \mathbf{E}_{1}^{\otimes} \times B S^{1} \rightarrow \mathbf{C y l}
$$

over $\Gamma$. Let $\mathcal{M}^{\otimes}$ be a symmetric monoidal $\infty$-category whose underlying category is $\mathcal{M}$. The restriction along $\mathbf{E}_{1}^{\otimes} \times B S^{1} \rightarrow \mathbf{C y l}$ induces a functor $z^{*}: \operatorname{Alg}_{\mathbf{C y l}}\left(\mathcal{M}^{\otimes}\right) \rightarrow \operatorname{Alg}_{\mathbf{E}_{1}^{\otimes} \times B S^{1}}\left(\mathcal{M}^{\otimes}\right)$.

Proposition 4.16. The functor

$$
z^{*}: \operatorname{Alg}_{\mathbf{C y l}}\left(\mathcal{M}^{\otimes}\right) \rightarrow \operatorname{Alg}_{\mathbf{E}_{1}^{\otimes} \times B S^{1}}\left(\mathcal{M}^{\otimes}\right)
$$

is an equivalence of $\infty$-categories.

Observe that Mult $\overline{\mathrm{Cyl}}\left(C^{\sqcup n} \sqcup C_{M}, C_{M}\right)$ is the singular complex of the space which is homeomorphic to Mult $_{\bar{E}_{1}}\left(D_{1}^{\sqcup n} \sqcup M, M\right) \times\left(S^{1}\right)^{\times n+1}$. As in the case of $\mathbf{E}_{1}^{\otimes} \times B S^{1} \rightarrow \mathbf{C y l}$, using Mult $_{\bar{E}_{1}}\left(D_{1}^{\sqcup n} \sqcup M, M\right) \times S^{1} \rightarrow \operatorname{Mult}_{\bar{E}_{1}}\left(D_{1}^{\sqcup n} \sqcup M, M\right) \times\left(S^{1}\right)^{\times n+1}$ induced by the diagonal map $S^{1} \rightarrow\left(S^{1}\right)^{\times n+1}$ we obtain a morphism $\bar{z}: \overline{\mathbf{E}}_{1}^{\otimes} \times B S^{1} \rightarrow \overline{\mathbf{C y l}}$ over $\Gamma$. The restriction along $\bar{z}$ gives rise to $\bar{z}^{*}: \operatorname{Alg} \overline{\mathbf{C y l}}\left(\mathcal{M}^{\otimes}\right) \rightarrow \operatorname{Alg}_{\overline{\mathbf{E}}_{1}^{\otimes} \times B S^{1}}\left(\mathcal{M}^{\otimes}\right)$. 
Proposition 4.17. The functor

$$
\bar{z}^{*}: \quad \operatorname{Alg} \overline{\mathbf{C y l}}\left(\mathcal{M}^{\otimes}\right) \rightarrow \operatorname{Alg}_{\overline{\mathbf{E}}_{1}^{\otimes} \times B S^{1}}\left(\mathcal{M}^{\otimes}\right)
$$

is an equivalence of $\infty$-categories.

Corollary 4.18. There are canonical equivalences of $\infty$-categories

$$
\begin{aligned}
\operatorname{Alg}_{\mathbf{C y l}}\left(\mathcal{M}^{\otimes}\right) & \simeq \operatorname{Alg}_{\mathbf{E}_{1}^{\otimes} \times B S^{1}}\left(\mathcal{M}^{\otimes}\right) \simeq \operatorname{Alg}_{\mathbf{E}_{1}}\left(\operatorname{Fun}\left(B S^{1}, \mathcal{M}\right)^{\otimes}\right) \\
& \simeq \operatorname{Alg}_{\text {Assoc }}\left(\operatorname{Fun}\left(B S^{1}, \mathcal{M}\right)^{\otimes}\right)
\end{aligned}
$$

and

$$
\begin{aligned}
\operatorname{Alg} \overline{\overline{\mathbf{C y}}_{\mathbf{l}}}\left(\mathcal{M}^{\otimes}\right) & \simeq \operatorname{Alg}_{\overline{\mathbf{E}}_{1}^{\otimes} \times B S^{1}}\left(\mathcal{M}^{\otimes}\right) \simeq \operatorname{Alg}_{\overline{\mathbf{E}}_{1}}\left(\operatorname{Fun}\left(B S^{1}, \mathcal{M}\right)^{\otimes}\right) \\
& \simeq \operatorname{LMod}\left(\operatorname{Fun}\left(B S^{1}, \mathcal{M}\right)^{\otimes}\right) .
\end{aligned}
$$

Here LMod $\left(\operatorname{Fun}\left(B S^{1}, \mathcal{M}\right)^{\otimes}\right)=\operatorname{Alg}_{\mathcal{L M}} \otimes\left(\operatorname{Fun}\left(B S^{1}, \mathcal{M}\right)^{\otimes}\right)$.

Proof. It follows from Lemma 4.14, Propositions 4.16 and 4.17, and Remark 4.13.

The proof of Proposition 4.17 is similar to Proposition 4.16. We prove Proposition 4.16.

Proof of Proposition 4.16. We use the notion of a weak approximation in the sense of [25, Definition 2.3.3.6] (since $z: \mathbf{E}_{1}^{\otimes} \times B S^{1} \rightarrow \mathbf{C y l}$ is not an equivalence of $\infty$-operads, it is necessary to use more delicate notion). According to [25, Theorem 2.3.3.23], if two conditions

(i) $\mathbf{E}_{1}^{\otimes} \times B S^{1} \rightarrow \mathbf{C y l}$ is a weak approximation,

(ii) $z: \mathbf{E}_{1}^{\otimes} \times B S^{1} \rightarrow \mathbf{C y l}$ induces an equivalence between the fiber over $\langle 1\rangle$

hold, then $z^{*}: \operatorname{Alg}_{\mathbf{C y l}}\left(\mathcal{M}^{\otimes}\right) \rightarrow \operatorname{Alg}_{\mathbf{E}_{1}^{\otimes} \times B S^{1}}\left(\mathcal{M}^{\otimes}\right)$ is an equivalence. We first prove (ii). Let $z_{\langle 1\rangle}:\left(\mathbf{E}_{1}^{\otimes} \times B S^{1}\right)_{\langle 1\rangle} \rightarrow \mathbf{C y l}_{\langle 1\rangle}$ be the map of fibers over $\langle 1\rangle$. Both fibers consist of a unique object (here we denote it by $*$ whose mapping $\operatorname{space} \operatorname{Map}(*, *)$ is (homotopy) equivalent to $S^{1}$ ). Taking into account our construction of $Z:\left(\mathrm{E}_{1} \times B S^{1}\right)_{\Delta} \rightarrow \mathrm{Cyl}_{\Delta}$, we see that $z_{\langle 1\rangle}$ is a homotopy equivalence $B S^{1} \rightarrow B S^{1}$. This proves (ii). Next we will prove (i). Let $p: \mathbf{E}_{1}^{\otimes} \times B S^{1} \rightarrow \Gamma$ be the projection. Let $\operatorname{Tup}_{n}$ be the subcategory of $\Gamma_{/\langle n\rangle}$ whose objects are active morphisms $\langle m\rangle \rightarrow\langle n\rangle$ and whose morphisms are equivalences. According to a criterion [25, Proposition 2.3.3.14], to prove (i), it is enough to prove that for any $X \in \mathbf{E}_{1}^{\otimes} \times B S^{1}$ with $\langle n\rangle=p(X)$, z induces a weak homotopy equivalence

$$
u: A(X):=\left(\mathbf{E}_{1}^{\otimes} \times B S^{1}\right)_{/ X} \times_{\Gamma_{/\langle n\rangle}} \operatorname{Tup}_{n} \rightarrow \mathbf{C y l}_{/ z(X)} \times_{\Gamma_{/\langle n\rangle}} \operatorname{Tup}_{n}=: B(z(X)) .
$$

Note that both domain and target are $\infty$-categories. Consequently, it will suffice to show that $u$ is a categorical equivalence. Clearly, $u$ is essentially surjective. We prove that $u$ is fully faithful. The general case is essentially the same as the case $n=1$ except for a more complicated notation, so that we treat the case of $n=1$. We think of $D_{1}$ as the unique object of $\left(\mathbf{E}_{1}^{\otimes} \times B S^{1}\right)_{\langle 1\rangle}$. Also, we write $D_{1}^{m}$ for the unique object of the fiber $\left(\mathbf{E}_{1}^{\otimes} \times B S^{1}\right)_{\langle m\rangle}$ over $\langle m\rangle$ (namely, $D_{1}=D_{1}^{1}$ ). Let $f: D_{1}^{m} \rightarrow D_{1}$ be a map in $\mathbf{E}_{1}^{\otimes} \times B S^{1}$ lying over an active morphism $\alpha:\langle m\rangle \rightarrow\langle 1\rangle$ of $\Gamma$. We regard $f$ as the product $g \times h:(0,1)^{\sqcup m} \times S^{1} \rightarrow(0,1) \times S^{1}$ of a rectilinear map $\phi:(0,1)^{\sqcup m} \rightarrow(0,1)$ and a rectilinear map $h: S^{1} \rightarrow S^{1}$. Let $f^{\prime}: D_{1}^{m} \rightarrow D_{1}$ be another map in $\mathbf{E}_{1}^{\otimes} \times B S^{1}$ lying over $\alpha$. We have

$$
\begin{aligned}
\operatorname{Map}_{A\left(D_{1}\right)}\left(f, f^{\prime}\right) & \simeq \operatorname{Map}_{\mathbf{E}_{1}^{\otimes} \times B S^{1}}^{\mathrm{eq}}\left(D_{1}^{m}, D_{1}^{m}\right) \times_{\operatorname{Map}_{\mathbf{E}_{1}^{\otimes} \times B S^{1}}\left(D_{1}^{m}, D_{1}\right)}\{f\} \\
& \simeq\left(S^{1} \times \Sigma_{m}\right) \times{ }_{\left(S^{1} \times \Sigma_{m}\right)}\{f\},
\end{aligned}
$$


where $\operatorname{Map}_{\mathbf{E}_{1}^{\otimes} \times B S^{1}}^{\mathrm{eq}}\left(D_{1}^{m}, D_{1}^{m}\right)$ is the full subcategory of $\operatorname{Map}_{\mathbf{E}_{1}^{\otimes} \times B S^{1}}\left(D_{1}^{m}, D_{1}^{m}\right)$ spanned by equivalences, and $\operatorname{Map}_{\mathbf{E}_{1}^{\otimes} \times B S^{1}}^{\mathrm{eq}}\left(D_{1}^{m}, D_{1}^{m}\right) \rightarrow \operatorname{Map}_{\mathbf{E}_{1}^{\otimes} \times B S^{1}}\left(D_{1}^{m}, D_{1}\right)$ is induced by the composition with $f^{\prime}$. Here $\Sigma_{m}$ denotes the symmetric group (which comes from permutations of components). Thus, the mapping space is contractible because $\left(S^{1} \times \Sigma_{m}\right) \rightarrow\left(S^{1} \times \Sigma_{m}\right)$ is an equivalence. Next, we regard the color $C$ in $\mathrm{Cyl}$ as an object in $\mathbf{C y l}$ that lies over $\langle 1\rangle$. We denote by $C^{m}$ the unique object of Cyl that lies over $\langle m\rangle$. Let $z(f), z\left(f^{\prime}\right): C^{m} \rightarrow C$ be the images of $f$ and $f^{\prime}$, respectively. Then we have equivalences in $\mathcal{S}$

$$
\begin{aligned}
& \operatorname{Map}_{B(C)}\left(z(f), z\left(f^{\prime}\right)\right) \simeq \operatorname{Map}_{\mathbf{C y l}}^{\mathrm{eq}}\left(C^{m}, C^{m}\right) \times_{\operatorname{Map}_{\mathbf{C y l}}\left(C^{m}, C\right)}\{z(f)\}
\end{aligned}
$$

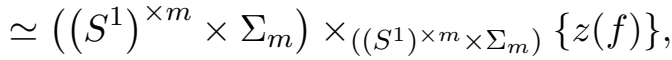

where $\operatorname{Map}_{\mathbf{C y l}}^{\mathrm{eq}}\left(C^{m}, C^{m}\right)$ is the full subcategory of $\operatorname{Map}_{\mathbf{C y l}}\left(C^{m}, C^{m}\right)$ spanned by equivalences. It follows from the canonical equivalence $\left(S^{1}\right)^{\times m} \times \Sigma_{m} \rightarrow\left(S^{1}\right)^{\times m} \times \Sigma_{m}$ that $\operatorname{Map}_{B(C)}\left(z(f), z\left(f^{\prime}\right)\right)$ is contractible. Thus, $\operatorname{Map}_{A\left(D_{1}\right)}\left(f, f^{\prime}\right) \rightarrow \operatorname{Map}_{B(C)}\left(z(f), z\left(f^{\prime}\right)\right)$ is an equivalence. We conclude that $u$ is a categorical equivalence.

4.3. Let $\mathcal{M}^{\otimes}$ be a symmetric monoidal $\infty$-category. If $\mathbf{P}^{\otimes}$ and $\mathbf{Q}^{\otimes}$ are small $\infty$-operads and $c: \mathbf{P}^{\otimes} \rightarrow \mathbf{Q}^{\otimes}$ is a morphism of $\infty$-operads (over $\Gamma$ ), then we denote by $c^{*}: \operatorname{Alg}_{\mathbf{Q}}(\mathcal{M}) \rightarrow$ $\operatorname{Alg}_{\mathbf{P}}(\mathcal{M})$ the restriction/forgetful functor along $c$. If there exists a left adjoint of $c^{*}$, we denote it by $c_{!}: \operatorname{Alg}_{\mathbf{P}}(\mathcal{M}) \rightarrow \operatorname{Alg}_{\mathbf{Q}}(\mathcal{M})$. If $\mathcal{M}^{\otimes}$ admits small colimits and its monoidal multiplication functor $\mathcal{M} \times \mathcal{M} \rightarrow \mathcal{M}$ preserves small colimits separately in each variable, then there exists a left adjoint $c_{\text {! }}$, cf. [25, Corollary 3.1.3.5].

The diagram DCyl $\stackrel{a}{\leftarrow}$ Cyl $\stackrel{b}{\rightarrow} \overline{\text { Cyl }}$ induces $a^{*}: \operatorname{Alg}_{\text {DCyl }}(\mathcal{M}) \rightarrow \operatorname{Alg}_{\mathbf{C y l}}(\mathcal{M})$ and $b^{*}:$ $\operatorname{Alg}_{\overline{\mathbf{C y l}}}(\mathcal{M}) \rightarrow \operatorname{Alg}_{\mathbf{C y l}}(\mathcal{M})$. It gives rise to the fiber product $\operatorname{Alg}_{\mathbf{D C y l}}(\mathcal{M}) \times_{\operatorname{Alg}_{\mathbf{C y l}}(\mathcal{M})} \operatorname{Alg}_{\overline{\mathbf{C y l}} \mathbf{l}}(\mathcal{M})$. We also have the inclusions DCyl $\rightarrow \overline{\mathbf{D C y l}}$ and $\overline{\mathbf{C y l}} \rightarrow \overline{\mathbf{D C y l}}$. Then the restriction/forgetful functors induce

$$
\operatorname{Alg} \overline{\mathbf{D C y l}}(\mathcal{M}) \rightarrow \operatorname{Alg}_{\mathbf{D C y l}}(\mathcal{M}) \times \operatorname{Alg}_{\mathbf{C y l}}(\mathcal{M}) \operatorname{Alg} \overline{\mathbf{C y l}}(\mathcal{M})
$$

Now suppose that $\mathcal{M}^{\otimes}$ admits small colimits and the tensor product functor $\mathcal{M} \times \mathcal{M} \rightarrow \mathcal{M}$ preserves small colimits separately in each variable. Let $i: \mathbf{E}_{2}^{\otimes} \rightarrow \mathbf{D C y l}$ be the canonical inclusion. We then have an adjoint pair

$$
i_{!}: \operatorname{Alg}_{\mathbf{E}_{2}}(\mathcal{M}) \rightleftarrows \operatorname{Alg}_{\mathbf{D C y l}}(\mathcal{M}): i^{*}
$$

where $i_{\text {! }}$ is fully faithful (indeed, the left adjoint is given by the operadic left Kan extension so that the unit map of the adjunction is the identity). We write $\operatorname{Alg}_{\mathbf{D C y l}}^{\mathbf{D}}(\mathcal{M})$ for the essential image of $i_{!}$. Note that $\operatorname{Alg}_{\mathbf{E}_{2}}(\mathcal{M}) \simeq \operatorname{Alg}_{\mathbf{D C y l}}^{\mathbf{D}}(\mathcal{M})$. We set $\operatorname{Alg}_{\overline{\mathbf{D C y l}}}^{\mathbf{D}}(\mathcal{M}):=\operatorname{Alg}_{\overline{\mathbf{D C y l}}}(\mathcal{M}) \times_{\operatorname{Alg}_{\mathbf{D C y l}}(\mathcal{M})}$ $\operatorname{Alg}_{\mathbf{D C y l}}^{\mathbf{D}}(\mathcal{M})$.

Proposition 4.19. Suppose that $q: \mathcal{M}^{\otimes} \rightarrow \Gamma$ is a symmetric monoidal $\infty$-category such that the underlying $\infty$-category $\mathcal{M}$ has small colimits and the tensor product functor $\otimes: \mathcal{M} \times \mathcal{M} \rightarrow \mathcal{M}$ preserves small colimits separately in each variable. Then the functor

$$
\operatorname{Alg}_{\overline{\mathbf{D C y l}}}(\mathcal{M}) \rightarrow \operatorname{Alg}_{\mathbf{D C y l}}(\mathcal{M}) \times \operatorname{Alg}_{\mathbf{C y l}}(\mathcal{M}) \operatorname{Alg}_{\overline{\mathbf{C y l}}}(\mathcal{M})
$$

is an equivalence of $\infty$-categories. Moreover, it induces an equivalence of $\infty$-categories

$$
\operatorname{Alg} \frac{\mathbf{D}}{\mathbf{D C y l}}(\mathcal{M}) \stackrel{\sim}{\rightarrow} \operatorname{Alg}_{\mathbf{D C y l}}^{\mathbf{D}}(\mathcal{M}) \times \operatorname{Alg}_{\mathbf{C y l}}(\mathcal{M}) \operatorname{Alg} \overline{\mathbf{C y l}_{\mathbf{l}}}(\mathcal{M})
$$


Proposition 4.20. The restriction along the inclusion $\mathbf{K S} \rightarrow \overline{\mathbf{D C y l}}$ induces an equivalence of $\infty$-categories

$$
\operatorname{Alg} \frac{\mathbf{D}}{\mathbf{D C y l}}(\mathcal{M}) \rightarrow \operatorname{Alg}_{\mathbf{K S}}(\mathcal{M})
$$

Now we consider the diagram

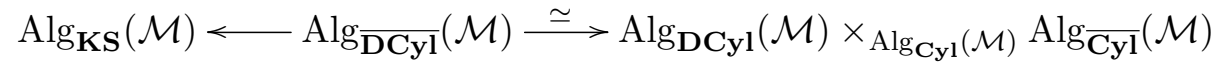

and its restriction to $\operatorname{Alg} \frac{\mathbf{D}}{\mathbf{D C y l}}(\mathcal{M})$.

Corollary 4.21. We have canonical categorical equivalences

$$
\operatorname{Alg}_{\mathbf{E}_{2}}(\mathcal{M}) \times_{\operatorname{Alg}_{\mathbf{C y l}}(\mathcal{M})} \operatorname{Alg}_{\overline{\mathbf{C y l}}}(\mathcal{M}) \simeq \operatorname{Alg}_{\mathbf{D C y l}}^{\mathbf{D}}(\mathcal{M}) \times_{\operatorname{Alg}_{\mathbf{C y l}}(\mathcal{M})} \operatorname{Alg}_{\overline{\mathbf{C y l}}}(\mathcal{M}) \simeq \operatorname{Alg}_{\mathbf{K S}}(\mathcal{M})
$$

Moreover, by Corollary 4.18, the $\infty$-category on the left-hand side is equivalent to

$$
\operatorname{Alg}_{\mathbf{E}_{2}}(\mathcal{M}) \times \operatorname{Alg}_{\mathbf{E}_{1}}\left(\operatorname{Fun}\left(B S^{1}, \mathcal{M}\right)\right) \operatorname{LMod}\left(\operatorname{Fun}\left(B S^{1}, \mathcal{M}\right)\right),
$$

where $\operatorname{Alg}_{\mathbf{E}_{2}}(\mathcal{M}) \rightarrow \operatorname{Alg}_{\mathbf{E}_{1}}\left(\operatorname{Fun}\left(B S^{1}, \mathcal{M}\right)\right)$ is the composite

$$
\operatorname{Alg}_{\mathbf{E}_{2}}(\mathcal{M}) \stackrel{i_{1}}{\simeq} \operatorname{Alg}_{\mathbf{D}}^{\mathbf{D} \mathbf{y} \mathbf{l}}(\mathcal{M}) \rightarrow \operatorname{Alg}_{\mathbf{C y l}}(\mathcal{M}) \simeq \operatorname{Alg}_{\mathbf{E}_{1}}\left(\operatorname{Fun}\left(B S^{1}, \mathcal{M}\right)\right)
$$

In particular, we have an equivalence of $\infty$-categories

$$
\operatorname{Alg}_{\mathbf{K S}}(\mathcal{M}) \simeq \operatorname{Alg}_{\mathbf{E}_{2}}(\mathcal{M}) \times \operatorname{Alg}_{\mathbf{E}_{1}}\left(\operatorname{Fun}\left(B S^{1}, \mathcal{M}\right)\right) \operatorname{LMod}\left(\operatorname{Fun}\left(B S^{1}, \mathcal{M}\right)\right)
$$

This equivalence commutes with projections to $\operatorname{Alg}_{\mathbf{E}_{2}}(\mathcal{M})$ in the natural way.

The proof of Proposition 4.19 requires Lurie-Barr-Beck theorem [25, Corollary 4.7.3.16]. Let us consider the comutative diagram

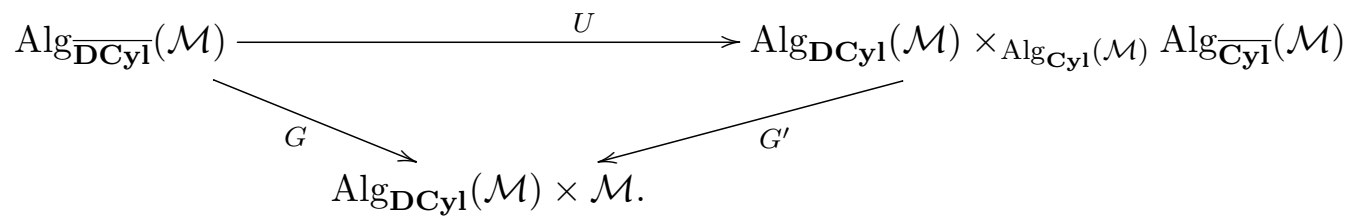

The functor $U$ is the functor in the statement in Proposition 4.19, and $G$ is determined by the forgetful/restriction functor $\operatorname{Alg}_{\overline{\mathbf{D C y l}}}(\mathcal{M}) \rightarrow \operatorname{Alg}_{\mathbf{D C y l}}(\mathcal{M})$ and the functor $\operatorname{Alg}_{\overline{\mathbf{D C y l}}}(\mathcal{M}) \rightarrow \mathcal{M}$ given by the evaluation at $C_{M}$. The functor $G^{\prime}$ is determined by the first projection and the functor $\operatorname{Alg}_{\overline{\mathbf{C y l}}}(\mathcal{M}) \rightarrow \mathcal{M}$ given by the evaluation at $C_{M}$. According to Lurie-Barr-Beck theorem [25, Corollary 4.7.3.16], $U$ is a categorical equivalence if the following conditions are satisfied:

(i) The functors $G$ and $G^{\prime}$ admit left adjoints $F$ and $F^{\prime}$, respectively.

(ii) $\operatorname{Alg} \overline{\mathbf{D C y l}}(\mathcal{M})$ admits geometric realizations of simplicial objects, which are preserved by $G$.

(iii) $\operatorname{Alg}_{\mathbf{D C y l}}(\mathcal{M}) \times \operatorname{Alg}_{\mathbf{C y l}}(\mathcal{M}) \operatorname{Alg} \overline{\mathbf{C y l}_{\mathbf{l}}}(\mathcal{M})$ admits geometric realizations of simplicial objects, which are preserved by $G^{\prime}$.

(iv) The functors $G$ and $G^{\prime}$ are conservative.

(v) For any object $X$ in $\operatorname{Alg}_{\mathbf{D C y l}}(\mathcal{M}) \times \mathcal{M}$, the unit map $X \rightarrow G F(X) \simeq G^{\prime} U F(X)$ induces an equivalence $G^{\prime} F^{\prime}(X) \rightarrow G F(X)$ in $\operatorname{Alg}_{\mathbf{D C y l}}(\mathcal{M}) \times \mathcal{M}$. 
Proof of Proposition 4.19. We will prove the conditions (i), (ii), (iii), (iv), (v).

A morphism $f$ in $\operatorname{Alg}_{\overline{\mathbf{D C y l}}}(\mathcal{M})$ is an equivalence if and only if the evaluations at $D, C, C_{M}$ are equivalences. Similarly, a morphism $f$ in $\operatorname{Alg}_{\mathbf{D C y l}}(\mathcal{M})$ is an equivalence if and only if the evaluations at objects $D, C$ are equivalences. It follows that $G$ is conservative. Similarly, we see that $G^{\prime}$ is conservative. Hence (iv) is proved. The conditions (ii) and (iii) follow from the existence and the compatibility of sifted colimits [25, Proposition 3.2.3.1] and the conservativity in (iv). (If $\mathcal{M}^{\otimes}$ is a presentably symmetric monoidal $\infty$-category, the condition (i) follows from adjoint functor theorem since $G$ and $G^{\prime}$ preserve small limit and filtered colimit. We prove (i) in full generality below.)

We prove the conditions (v) and (i). For this purpose, we first consider the left adjoint $F^{\prime}$ of $G^{\prime}$. The values under $F^{\prime}$ can be described in terms of operadic colimits if we assume the existence of a left adjoint $F^{\prime}$. Let $j: \mathbf{C y l} \rightarrow \overline{\mathbf{C y l}}$ be the canonical inclusion. Let $j^{\prime}: \operatorname{Triv}^{\otimes} \rightarrow \overline{\mathbf{C y l}}$ be a morphism from the trivial $\infty$-operad to $\overline{\mathbf{C y l}}$ that is determined by $C_{M}$. Let $\mathbf{C y l} \boxplus \operatorname{Triv}^{\otimes}$ denote the coproduct of $\mathbf{C y l}$ and $\operatorname{Triv}^{\otimes}$. Namely, it is a coproduct of $\mathbf{C y l}$ and $\operatorname{Triv}^{\otimes}$ in the $\infty$-category of $\infty$-operads, but we use its explicit construction in [25, Construction 2.2.3.3]. By the universal property, the morphism $j$ and $j^{\prime}$ induces $k: \mathbf{C y l} \boxplus \operatorname{Triv}^{\otimes} \rightarrow \overline{\mathbf{C y l}}$. By $[25$, Corollary 3.1.3.5], we have an adjoint pair

$$
k_{!}: \quad \operatorname{Alg}_{\mathbf{C y l}}(\mathcal{M}) \times \mathcal{M} \simeq \operatorname{Alg}_{\mathbf{C y l} \boxplus \operatorname{Triv}} \otimes(\mathcal{M}) \rightleftarrows \operatorname{Alg}_{\overline{\mathbf{C y l}}}(\mathcal{M}): k^{*}
$$

Here we use the canonical categorical equivalence $\operatorname{Alg}_{\text {Triv }}(\mathcal{M}) \stackrel{\sim}{\rightarrow} \mathcal{M}$. There are categorical fibrations $\operatorname{pr}_{1}: \operatorname{Alg}_{\mathbf{C y l}}(\mathcal{M}) \times \mathcal{M} \rightarrow \operatorname{Alg}_{\mathbf{C y l}}(\mathcal{M})$ and $\operatorname{Alg}_{\overline{\mathbf{C y l}}}(\mathcal{M}) \rightarrow \operatorname{Alg}_{\mathbf{C y l}}(\mathcal{M})$ induced by the inclusion $\mathbf{C y l} \hookrightarrow \overline{\mathbf{C y l}}$. The right adjoint $k^{*}$ commutes with these projections to $\operatorname{Alg}_{\mathbf{C y l}}(\mathcal{M})$. Let $Y: \mathbf{C y l} \boxplus \operatorname{Triv}^{\otimes} \rightarrow \mathcal{M}^{\otimes}$ be a map of $\infty$-operads, which we regard as an object $Y$ of $\operatorname{Alg}_{\mathbf{C y l} \boxplus \operatorname{Triv}} \otimes(\mathcal{M})$. For $A \in \overline{\mathbf{C y l}}$, the evaluation of $k_{!}(Y)$ at $A$ is an operadic $q$-colimit of the map

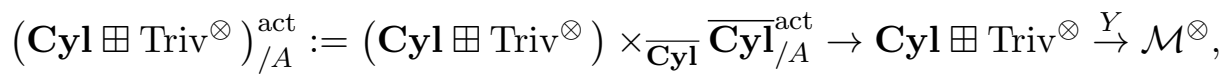

lying over $\left(\left(\mathbf{C y l} \boxplus \operatorname{Triv}^{\otimes}\right)_{/ A}^{\text {act }}\right)^{\triangleright} \rightarrow \Gamma$. See $[25$, Sections 3.1.1-3.1.3] for operadic left Kan extensions and operadic colimits. Note that $\operatorname{Mult}_{\overline{\mathrm{Cyl}}}\left(C^{\sqcup n} \sqcup C_{M}^{\sqcup m}, C\right)$ is the empty set for $m \neq 0$. Hence $\left(\mathbf{C y l} \boxplus \operatorname{Triv}^{\otimes}\right) \times \overline{\mathbf{C y l}}_{\mathbf{C y l}} \overline{\text { act }}_{C} \simeq \mathbf{C y l} \times \overline{\mathbf{C y l}}_{\overline{\mathbf{C y l}}_{/ C}}^{\text {act }}$ so that there is a final object determined by the identity $C \rightarrow C$. It follows that $\operatorname{pr}_{1}(Y) \rightarrow \operatorname{pr}_{1} k^{*} k_{!}(Y)$ is an equivalence in $\operatorname{Alg}_{\mathbf{C y l}}(\mathcal{M})$ for each $Y \in \operatorname{Alg}_{\mathbf{C y l} \boxplus \operatorname{Triv}} \otimes(\mathcal{M}) \simeq \operatorname{Alg}_{\mathbf{C y l}}(\mathcal{M}) \times \mathcal{M}$. Thus $\left(k_{!}, k^{*}\right)$ is an adjunction relative to $\operatorname{Alg}_{\mathbf{C y l}}(\mathcal{M})$. See [25, Definition 7.3.2.2] for the notion of relative adjunctions. The base change of $\left(k_{!}, k^{*}\right)$ along $\operatorname{Alg}_{\mathbf{D C y l}}(\mathcal{M}) \rightarrow \operatorname{Alg}_{\mathbf{C y l}}(\mathcal{M})$ gives rise to an adjunction

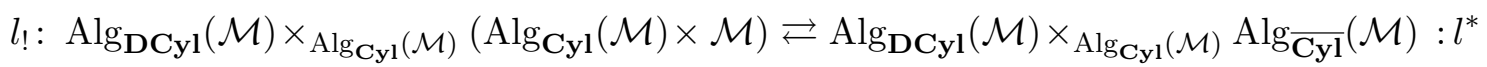

relative to $\operatorname{Alg}_{\mathbf{D C y l}}(\mathcal{M})$, where the right adjoint is $G^{\prime}$. This shows that there exists a left adjoint $F^{\prime}$ of $G^{\prime}$. In addition, $F^{\prime} \simeq l_{\text {! }}$. We denote informally by $X=(A, B, M)$ an object of $\operatorname{Alg}_{\mathbf{D C y l}}(\mathcal{M}) \times \mathcal{M} \simeq \operatorname{Alg}_{\mathbf{C y l} \mathbf{l} \boxplus \operatorname{Triv}} \otimes(\mathcal{M})$, where $(A, B) \in \operatorname{Alg}_{\mathbf{D C y l}}(\mathcal{M}), M \in \mathcal{M}, A$ is the restriction to $\mathbf{E}_{2}^{\otimes} \subset \mathbf{D C y l}$, and $B$ is the restriction to Cyl $\subset \mathbf{D C y l}$. We compute the image of $(A, B, M)$ under the left adjoint $l_{!}$. The left adjoint $F^{\prime}=l_{\text {! }}$ is induced by $k_{!}$so that $l_{!}(A, B, M)=\left((A, B), k_{!}(B, M)\right)$, where $\left((A, B), k_{!}(B, M)\right)$ indicates the object of the fiber product on the right-hand side. The pair $(B, M)$ is an object of $\operatorname{Alg}_{\mathbf{C y l}}(\mathcal{M}) \times \mathcal{M}$. Thus we will compute $k_{!}(B, M)$ in terms of the operadic left Kan extension; we describe it as a colimit of a certain diagram. Let $(B, M): \mathbf{C y l} \boxplus \operatorname{Triv}^{\otimes} \rightarrow \mathcal{M}^{\otimes}$ be a morphism of $\infty$-operads which corresponds to $(B, M) \in \operatorname{Alg}_{\mathbf{C y l}}(\mathcal{M}) \times \mathcal{M}$. Let $\mathbf{C y l} \boxplus \operatorname{Triv}^{\otimes} \rightarrow \overline{\mathbf{C y l}}$ be a morphism determined by $\mathbf{C y l} \rightarrow \overline{\mathbf{C y l}}$ and the morphism $\operatorname{Triv}^{\otimes} \rightarrow \overline{\mathbf{C y l}}$ classified by the object $C_{M}$ in the fiber $\overline{\mathbf{C y l}}_{\langle 1\rangle}$ 
(by using the same symbol $C_{M}$ we abuse notation). Let $\overline{\mathbf{C y l}}^{\text {act }}$ be the subcategory spanned by those morphisms whose images in $\Gamma$ are active (cf. [25, Definition 2.1.2.1]). Let us consider $\left(\mathbf{C y l} \boxplus \operatorname{Triv}^{\otimes}\right)_{/ C_{M}}^{\text {act }}=\left(\mathbf{C y l} \boxplus \operatorname{Triv}^{\otimes}\right) \times \overline{\mathbf{C y l}}_{\overline{\mathbf{C y l}}_{/}}^{\text {act }} C_{M}$. We have a morphism

$$
p: \quad K:=\left(\mathbf{C y l} \boxplus \operatorname{Triv}^{\otimes}\right)_{/ C_{M}}^{\text {act }} \rightarrow \mathbf{C y l} \boxplus \operatorname{Triv}^{\otimes} \stackrel{(B, M)}{\longrightarrow} \mathcal{M}^{\otimes}
$$

that extends $\left(\mathbf{C y} \mathbf{l} \boxplus \operatorname{Triv}^{\otimes}\right)_{/ C_{M}}^{\text {act }} \rightarrow \Gamma$. According to [25, Proposition 3.1.3.2], the operadic left Kan extension $k_{!}(B, M): \overline{\mathbf{C y l}} \rightarrow \mathcal{M}^{\otimes}$ of $k$ carries $C_{M}$ to a colimit of $p$, that is, the image of the cone point under an operadic $q$-colimit diagram $p^{\prime}: K^{\triangleright} \rightarrow \mathcal{M}^{\otimes}$ that extends $p$. Let $\star$ denote the unique object of $\operatorname{Triv}^{\otimes}$ lying over $\langle 1\rangle$. Let Sub be a category defined as follows [25, Definition 2.2.3.2]: The objects of Sub are triples $(\langle n\rangle, S, T)$ such that $\langle n\rangle \in \mathrm{Fin}_{*}$, and $S, T \subset\langle n\rangle$ are subsets such that $S \cap T=*$ and $S \cup T=\langle n\rangle$. A morphism $(\langle n\rangle, S, T) \rightarrow\left(\left\langle n^{\prime}\right\rangle, S^{\prime}, T^{\prime}\right)$ is a morphism $\langle n\rangle \rightarrow\langle n\rangle$ in Fin $_{*}$ such that $f(S) \subset S^{\prime}$ and $f(T) \subset T^{\prime}$. Let

$$
\left(\langle n\rangle, S, T, C^{n-1}=(C, \ldots, C), \star\right)
$$

be an object of $\mathbf{C y l} \boxplus \operatorname{Triv}^{\otimes}$ lying over $\langle n\rangle$ such that $(\langle n\rangle, S, T)$ is an object of Sub, $C^{n-1}$ is the unique object of $\mathbf{C y l}_{\langle n-1\rangle}$ (lying over $\langle n-1\rangle$ ), and $\star \in \operatorname{Triv}=\operatorname{Triv}_{\langle 1\rangle}^{\otimes}$. This presentation is based on the explicit construction of the coproducts in [25, Construction 2.2.3.3]. For our purpose below, we may assume that $T \subset\langle n\rangle$ is of the form $T=\{*, i\}$ so that by default $T$ in the above object is of the form $T=\{*, i\}$. The mapping space from $\left(\langle n\rangle, S, T, C^{n-1}, \star\right)$ to $\left(\langle m\rangle, S^{\prime}, T^{\prime}, C^{m-1}, \star\right)$ is given by

$$
\left(\coprod_{\substack{\alpha:\langle n\rangle \rightarrow\langle m\rangle \\ \alpha(S) \subset S^{\prime}, \alpha(T) \subset T^{\prime}}} \prod_{j \in\langle m\rangle \backslash T^{\prime}} \operatorname{Emb}^{\mathrm{rec}}\left(\left((0,1) \times S^{1}\right) \times \alpha^{-1}(j),(0,1) \times S^{1}\right)\right) \times *,
$$

which we regard as an object in $\mathcal{S}$, and $*$ indicates the contractible space which we regard as the mapping space from $\star$ to $\star$. Using this description we consider mapping spaces in $\left(\mathbf{C y l} \boxplus \operatorname{Triv}^{\otimes}\right)_{/ C_{M}}^{\text {act }}$. We abuse notation by writing $\left(\langle n\rangle, S, T, C^{n-1}, \star,\left(C^{n-1}, C_{M}\right) \rightarrow C_{M}\right)$ for an object of $K$, where $\left(\langle n\rangle, S, T, C^{n-1}, \star\right) \in \mathbf{C y l} \boxplus \operatorname{Triv}^{\otimes}$ and $\left(C, \ldots, C, C_{M}\right)=\left(C^{n-1}, C_{M}\right) \rightarrow$ $C_{M}$ is a morphism in $\overline{\mathbf{C y l}}^{\text {act }}$ lying over the active morphism $\langle n\rangle \rightarrow\langle 1\rangle$, where $\left(C^{n-1}, C_{M}\right)$ is a sequence of $n-1 C$ 's and a single $C_{M}$ which we regard as an object in $\overline{\mathbf{C y l}}_{\langle n\rangle}$. Now it is easy to compute the mapping space from $H=\left(\langle n\rangle, S, T, C^{n-1}, \star,\left(C^{n-1}, C_{M}\right) \stackrel{f}{\rightarrow} C_{M}\right)$ to $H^{\prime}=\left(\langle m\rangle, S^{\prime}, T^{\prime}, C^{m-1}, \star,\left(C^{m-1}, C_{M}\right) \stackrel{g}{\rightarrow} C_{M}\right)$ in $\left(\mathbf{C y l} \boxplus \operatorname{Triv}^{\otimes}\right) \times \overline{\mathbf{C y l}}_{\mathbf{C y l}^{\mathbf{l}}} \bar{C}_{M}$. We think of $f$ as an embedding $\left((0,1) \times S^{1}\right)^{\sqcup n-1} \sqcup(0,1) \times S^{1} \rightarrow(0,1) \times S^{1}$ that belongs to Mult $\overline{\mathbf{C y l}^{1}}\left(C^{\sqcup n-1} \sqcup\right.$ $C_{M}, C_{M}$ ) (namely, the restricition to the "right component" $(0,1) \times S^{1}$ is shrinking). Consider the restriction $\left((0,1) \times S^{1}\right)^{\sqcup n-1} \rightarrow(0,1) \times S^{1}$ and its projection $\bar{f}:(0,1)^{\sqcup n-1} \rightarrow(0,1)$ obtained by forgetting the $S^{1}$-factor, which is a rectilinear embedding. If we denote by $D_{1}^{n}$ the unique object in the fiber $\left(\mathbf{E}_{1}^{\otimes}\right)_{\langle n\rangle}$ over $\langle n\rangle \in \Gamma$, we can regard $\bar{f}$ as a map $D_{1}^{n-1} \rightarrow D_{1}:=D_{1}^{1}$ in $\mathbf{E}_{1}^{\otimes}$. Let $\operatorname{Map}_{\left(\mathbf{E}_{1}^{\otimes}\right)_{/ D_{1}}}(\bar{f}, \bar{g})$ be the discrete mapping space from $\bar{f}: D_{1}^{n-1} \rightarrow D_{1}$ to $\bar{g}: D_{1}^{m-1} \rightarrow D_{1}$. Given a morphism $H \rightarrow H^{\prime}$, by applying the same procedure to the induced morphism $C^{n-1} \rightarrow C^{m-1}$, we obtain a map $\operatorname{Map}_{K}\left(H, H^{\prime}\right) \rightarrow \operatorname{Map}_{\left(\mathbf{E}_{1}^{\otimes}\right)_{/ D_{1}}}(\bar{f}, \bar{g})$. Note that $\operatorname{Map}_{\overline{\mathbf{C y}} \mathbf{l}_{/ C_{M}}}\left(C_{M}, C_{M}\right)$ is contractible. Consequently, the restriction to the component $C_{M}$ gives rise to

$$
\operatorname{Map}_{K}\left(H, H^{\prime}\right) \rightarrow \operatorname{Map}_{\text {Triv }}(\star, \star) \times_{\operatorname{Map}_{\overline{\mathbf{C y l}}}\left(C_{M}, C_{M}\right)} \operatorname{Map}_{\overline{\mathbf{C y l}}_{/ C_{M}}^{\text {act }}}\left(C_{M}, C_{M}\right) \simeq * \times_{S^{1}} *=\mathbf{Z}
$$


Taking account of definitions of $\infty$-operads $\mathbf{C y l} \boxplus \operatorname{Triv}^{\otimes}$ and $\overline{\mathbf{C y l}}$, we see that

$$
\operatorname{Map}_{K}\left(H, H^{\prime}\right) \rightarrow \operatorname{Map}_{\left(\mathbf{E}_{1}^{\otimes}\right) / D_{1}}(\bar{f}, \bar{g}) \times \mathbf{Z}
$$

is an equivalence in $\mathcal{S}$. Let $L$ be the full subcategory of $K$ spanned by the single object

$$
\mathrm{Z}=\left(\langle 2\rangle, S \simeq\langle 1\rangle, T \simeq\langle 1\rangle, C, \star,\left(C, C_{M}\right) \stackrel{j}{\rightarrow} C_{M}\right)
$$

(a morphism $j$ is uniquely determined up to homotopy). We now claim that $L \subset K$ is cofinal. It will suffice to prove that for each $\mathrm{V} \in K$, the $\infty$-category $L \times_{K} K_{\mathrm{V}}$ / is weakly contractible, see [25, Definition 4.1.3.1]. Let $\mathrm{V}=\left(\langle n\rangle, S, T, C^{n-1}, \star,\left(C^{n-1}, C_{M}\right) \stackrel{f}{\rightarrow} C_{M}\right)$. By the above discussion about mapping spaces, a morphism $u: \mathrm{V} \rightarrow \mathbf{Z}$ is uniquely determined by $a \in \mathbf{Z}$ since $\operatorname{Map}_{\left(\mathbf{E}_{1}^{\otimes}\right) / D_{1}}(\bar{f}, \bar{j})$ is contractible. Let $u^{\prime}: \mathrm{V} \rightarrow \mathrm{Z}$ be another object of $L \times_{K} K_{\mathrm{V} /}$ that is determined by $a^{\prime} \in \mathbf{Z}$. Note that $\operatorname{Map}_{K}(\mathbf{Z}, \mathbf{Z}) \simeq \mathbf{Z}$ and the $\operatorname{composition} \operatorname{Map}_{K}(\mathbf{Z}, \mathbf{Z}) \times$ $\operatorname{Map}_{K}(\mathbf{V}, \mathbf{Z}) \simeq \mathbf{Z} \times \mathbf{Z} \rightarrow \operatorname{Map}_{K}(\mathbf{V}, \mathbf{Z}) \simeq \mathbf{Z}$ can be identified with the additive operation $+: \mathbf{Z} \times$ $\mathbf{Z} \rightarrow \mathbf{Z}$ (up to automorphisms of $\mathbf{Z}$ ). It follows that $\operatorname{Map}_{L \times_{K} K_{\mathrm{V}} /}\left(u, u^{\prime}\right)$ is a contractible space so that the $\infty$-category $L \times_{K} K_{\mathrm{V} /}$ is contractible. Let $p^{\prime}: K^{\triangleright} \rightarrow \mathcal{M}^{\otimes}$ be the operadic $q$-colimit diagram. Let $p^{\prime \prime}: K^{\triangleright} \rightarrow \mathcal{M}$ be the diagram obtained by a $q$-coCartesian natural transformation from $p^{\prime}$. Since we assume that $\otimes: \mathcal{M} \times \mathcal{M} \rightarrow \mathcal{M}$ preserves small colimits separately in each variable, then by [25, Propositions 3.1.1.15 and 3.1.1.16], $p^{\prime \prime}$ is a colimit diagram of $\left.p^{\prime \prime}\right|_{K}$ : $K \rightarrow \mathcal{M}$, and the image of the cone point under $p^{\prime \prime}$ is naturally equivalent to the image of the cone point under $p^{\prime}$. Since $L \subset K$ is cofinal, we have a canonical equivalence colim $\left.\left.p^{\prime \prime}\right|_{K} \simeq \operatorname{colim} p^{\prime \prime}\right|_{L}$.

Indeed, colim $\left.p^{\prime \prime}\right|_{K}$ is equivalent to $B \otimes M \otimes S^{1}$ (this computation is not necessary to the proof so that the reader may skip this paragraph, but it may be helpful to get feeling for the operadic left Kan extension $F^{\prime}$ ). By construction, the composite $L \hookrightarrow K \stackrel{p}{\rightarrow} \mathcal{M}^{\otimes}$ is equivalent to a contant diagram so that $\left.p^{\prime \prime}\right|_{L}: L \rightarrow \mathcal{M}$ is a contant diagram taking the value $B \otimes M$. Note that $\operatorname{Map}_{K}(\mathbf{Z}, \mathbf{Z})=\mathbf{Z}$ and there is a categorical equivalence $L \simeq B \mathbf{Z} \simeq S^{1}$. We deduce that colim $\left.p^{\prime \prime}\right|_{L} \simeq(B \otimes M) \otimes S^{1}$. Namely, the evaluation $F^{\prime}(A, B, M)\left(C_{M}\right)$ of $F^{\prime}(A, B, M)$ at $C_{M}$ is $\left.\left.\operatorname{colim} p^{\prime \prime}\right|_{K} \simeq \operatorname{colim} p^{\prime \prime}\right|_{L} \simeq(B \otimes M) \otimes S^{1}$. Another way to compute it is as follows. By Corollary 4.18, we have $\operatorname{Alg}_{\mathbf{C y l}}(\mathcal{M}) \simeq \operatorname{Alg}_{\mathrm{As}}\left(\operatorname{Fun}\left(B S^{1}, \mathcal{M}\right)\right)$ and $\operatorname{Alg}_{\overline{\mathbf{C y l}}}(\mathcal{M}) \simeq$ $\operatorname{LMod}\left(\operatorname{Fun}\left(B S^{1}, \mathcal{M}\right)\right)$. These equivalences commute with forgetful functors arising from the inclusions As $\rightarrow \mathcal{L} \mathcal{M}$ and $\mathbf{C y l} \rightarrow \overline{\mathbf{C y l}}$. The adjunction $\left(k_{!}, k^{*}\right)$ can be identified with the composite of adjunctions

$$
\begin{aligned}
\operatorname{Alg}_{\mathrm{As}}\left(\operatorname{Fun}\left(B S^{1}, \mathcal{M}\right)\right) \times \mathcal{M} & \rightleftarrows \operatorname{Alg}_{\mathrm{As}}\left(\operatorname{Fun}\left(B S^{1}, \mathcal{M}\right)\right) \times \operatorname{Fun}\left(B S^{1}, \mathcal{M}\right) \\
& \rightleftarrows \operatorname{LMod}\left(\operatorname{Fun}\left(B S^{1}, \mathcal{M}\right)\right),
\end{aligned}
$$

where the left adjunction is induced by the adjunction $\mathcal{M} \rightleftarrows \operatorname{Fun}\left(B S^{1}, \mathcal{M}\right)$ which consists of the forgetful functor Fun $\left(B S^{1}, \mathcal{M}\right) \rightarrow \mathcal{M}$ and the left adjoint free functor which sends $M$ to $S^{1} \otimes M$. The right adjoint in the right adjunction is given by the evaluation of the module objects $\operatorname{LMod}\left(\operatorname{Fun}\left(B S^{1}, \mathcal{M}\right)\right) \rightarrow \operatorname{Fun}\left(B S^{1}, \mathcal{M}\right)$ and $\operatorname{LMod}\left(\operatorname{Fun}\left(B S^{1}, \mathcal{M}\right)\right) \rightarrow \operatorname{Alg}_{\mathrm{As}}\left(\operatorname{Fun}\left(B S^{1}, \mathcal{M}\right)\right)$ induced by $\mathrm{As} \hookrightarrow \mathcal{L} \mathcal{M}$. The left adjoint functors carry $(B, M)$ to

$$
\left(B, B \otimes S^{1} \otimes M\right) \in \operatorname{LMod}\left(\operatorname{Fun}\left(B S^{1}, \mathcal{M}\right)\right) .
$$

Next we will consider $F(A, B, M)$. Let $r$ : DCyl $\boxplus$ Triv $^{\otimes} \rightarrow \overline{\mathbf{D C y l}}$ be a morphism of $\infty$-operads induced by DCyl $\hookrightarrow \overline{\mathbf{D C y l}}$ and $\operatorname{Triv}^{\otimes} \rightarrow \overline{\mathbf{D C y l}}$ determined by $C_{M} \in \overline{\mathbf{D C y l}}_{\langle 1\rangle}$ correpondings to the color $C_{M}$ (we slightly abuse notation again). By [25, Corollary 3.1.3.5], we have an adjunction $r_{!}: \operatorname{Alg}_{\mathbf{D C y l}}(\mathcal{M}) \times \mathcal{M} \rightleftarrows \operatorname{Alg}_{\overline{\mathbf{D C y l}}}(\mathcal{M}): r^{*}$. This shows that there exists a left adjoint $F=r$ ! of $G$. Consider

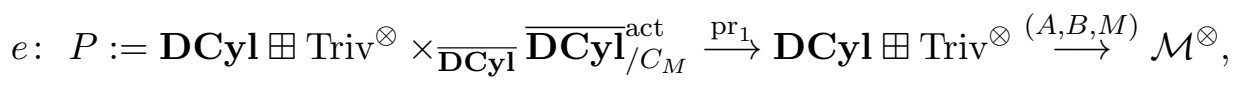


where the right arrow classifies the algebra object $(A, B, M) \in \operatorname{Alg}_{\mathbf{D C y l}}(\mathcal{M}) \times \mathcal{M}$. The evaluation of $r_{!}(A, B, M)$ at $C_{M}$ is a colimit of $e$, that is, the image of the cone point under an operadic $q$-colimit diagram $e^{\prime}: P^{\triangleright} \rightarrow \mathcal{M}^{\otimes}$ that extends $e$. The computation of the colimit of $e$ is similar to that of $p$. We infomally denote by $\left(\langle n\rangle, S, T, D^{d}, C^{c}, \star\right)$ an object of DCyl $\boxplus$ Triv $^{\otimes}$, where $\star \in$ Triv, and $\left(D^{d}, C^{c}\right)$ indicates the sequence of colors which consists of $d D$ 's and $c C$ 's which we regard as an object in $\mathbf{D C} \mathbf{y l}_{\langle n-1\rangle}(d+c=n-1)$. By abuse of notation, we write

$$
R=\left(\langle n\rangle, S, T, D^{d}, C^{c}, \star,\left(D^{d}, C^{c}, C_{M}\right) \stackrel{f}{\rightarrow} C_{M}\right)
$$

for an object of $P$, where $f:\left(D^{d}, C^{c}, C_{M}\right) \rightarrow C_{M}$ is a morphism in $\overline{\mathbf{D C y l}}^{\text {act }}$ that lies over the active morphism $\langle n\rangle \rightarrow\langle 1\rangle$. We compute the mapping space from $R$ to another object

$$
R^{\prime}=\left(\langle m\rangle, S^{\prime}, T^{\prime}, D^{d^{\prime}}, C^{c^{\prime}}, \star,\left(D^{d^{\prime}}, C^{c^{\prime}}, C_{M}\right) \stackrel{g}{\rightarrow} C_{M}\right)
$$

Given a morphism $\phi: R \rightarrow R^{\prime}$ we have the induced morphism $\left(D^{d}, C^{c}, C_{M}\right) \rightarrow\left(D^{d^{\prime}}, C^{c^{\prime}}, C_{M}\right)$ in $\overline{\mathbf{D C y l}}_{/ C_{M}}^{\text {act }}$. Notice that it is given by the union of $\left(D^{d}, C^{c}\right) \rightarrow\left(D^{d^{\prime}}, C^{c^{\prime}}\right)$ and $C_{M} \rightarrow$ $C_{M}$ over $C_{M}$. Moreover, we can think of $\left(D^{d}, C^{c}\right) \rightarrow\left(D^{d^{\prime}}, C^{c^{\prime}}\right)$ as a rectilinear embedding $\left((0,1)^{2}\right)^{\sqcup d} \sqcup\left((0,1) \times S^{1}\right)^{\sqcup c} \rightarrow\left((0,1)^{2}\right)^{\sqcup d^{\prime}} \sqcup\left((0,1) \times S^{1}\right)^{\sqcup c^{\prime}}$ over $(0,1) \times S^{1}$. In this way, we obtain the induced morphism

$$
\operatorname{Map}_{P}\left(R, R^{\prime}\right) \rightarrow \operatorname{Map}_{\mathbf{D C y}}^{\text {act }} \underset{\text { act }}{ }\left(\left(D^{d}, C^{c}\right),\left(D^{d^{\prime}}, C^{c^{\prime}}\right)\right) .
$$

As in the case of $K$, the restriction to $C_{M}$ gives rise to a morphism

$$
\operatorname{Map}_{P}\left(R, R^{\prime}\right) \rightarrow \operatorname{Map}_{\text {Triv }}(\star, \star) \times_{\operatorname{Map}_{\overline{\mathbf{D C y l}}}\left(C_{M}, C_{M}\right)} \operatorname{Map}_{\overline{\mathbf{D C y l}}}{ }_{/ C_{M}}^{\text {act }}\left(C_{M}, C_{M}\right) \simeq * \times_{S^{1}} *=\mathbf{Z} .
$$

It gives rise to an equivalence in $\mathcal{S}$ :

$$
\operatorname{Map}_{P}\left(R, R^{\prime}\right) \rightarrow \operatorname{Map}_{\mathbf{D C y}}{ }_{/ C}^{\text {act }}\left(\left(D^{d}, C^{c}\right),\left(D^{d^{\prime}}, C^{c^{\prime}}\right)\right) \times \mathbf{Z} .
$$

Let $Q \subset P$ be the full subcategory spanned by $\mathrm{Z}$ which we think of as an object of $P$ in the obvious way. As in the case of $K$, using the above description of $\operatorname{Map}_{P}\left(R, R^{\prime}\right)$ we see that for any $\mathrm{V} \in P, Q \times_{P} P_{\mathrm{V} /}$ is weakly contractible so that $Q \subset P$ is cofinal. Let $e^{\prime}: P^{\triangleright} \rightarrow \mathcal{M}^{\otimes}$ be an operadic $q$-colimit diagram that extends $e$. Let $e^{\prime \prime}: P^{\triangleright} \rightarrow \mathcal{M}=\mathcal{M}_{\langle 1\rangle}$ be the diagram obtained by a $q$-coCartesian natural transformation from $e^{\prime}$. Then the image of the cone point under $e^{\prime}$ is colim $\left.\left.e^{\prime \prime}\right|_{P} \simeq \operatorname{colim} e^{\prime \prime}\right|_{Q}$. (We can also deduce that $r_{!}(A, B, M)\left(C_{M}\right)=F(A, B, M)\left(C_{M}\right)$ is $B \otimes M \otimes S^{1} \in \mathcal{M}$ in the same way as described above.)

The projection of $G^{\prime} F^{\prime}(A, B, M) \rightarrow G F(A, B, M)$ to $\mathcal{M}$ is the canonical map colim $\left.p^{\prime \prime}\right|_{K} \rightarrow$ colim $\left.e^{\prime \prime}\right|_{P}$. We note that the canonical functor $K \rightarrow P$ induces an equivalence $L \stackrel{\sim}{\rightarrow} Q$. Consequently, colim $\left.\left.p^{\prime \prime}\right|_{K} \rightarrow \operatorname{colim} e^{\prime \prime}\right|_{P}$ can be identified with the equivalence colim $\left.\left.p^{\prime \prime}\right|_{L} \stackrel{\sim}{\rightarrow} \operatorname{colim} e^{\prime \prime}\right|_{Q}$. Next we consider the projection of $G^{\prime} F^{\prime}(A, B, M) \rightarrow G F(A, B, M)$ to $\operatorname{Alg}_{\text {DCyl }}(\mathcal{M})$. Taking into account the equivalences DCyl $\boxplus \operatorname{Triv}^{\otimes} \times_{\overline{\mathbf{D C y l}}} \overline{\mathbf{D C y l}}_{/ D}^{\text {act }} \simeq \mathbf{D C y l} \times \overline{\mathbf{D C y l}}_{\overline{\mathbf{D C y l}}} \overline{\mathbf{D C t}}_{D}^{\text {act }}$ and $\mathbf{D C y l} \boxplus$

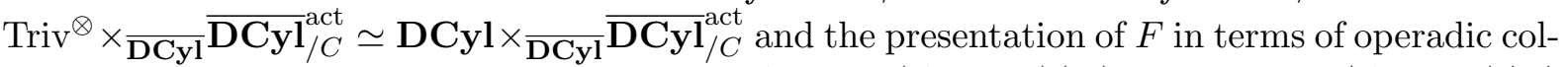
imits, we see that the evaluations of unit maps $A \rightarrow G F(A, B, M)(D)$ and $B \rightarrow G F(A, B, M)(C)$ are equivalence so that the evaluations $A \simeq G^{\prime} F^{\prime}(A, B, M)(D) \rightarrow G F(A, B, M)(D)$ and $B \simeq$ $G^{\prime} F^{\prime}(A, B, M)(C) \rightarrow G F(A, B, M)(C)$ of $G^{\prime} F^{\prime}(A, B, M) \rightarrow G F(A, B, M)$ are also equivalences. Consequently, $G^{\prime} F^{\prime}(A, B, M) \rightarrow G F(A, B, M)$ is an equivalence since evaluations at $D, C$, and the projection to $\mathcal{M}$ are equivalences. This proves (v). We also have proved the existence of $F$ and $F^{\prime}$, that is, (i). The final assertion is clear. 
Proof of Proposition 4.20. The inclusion $j: \mathbf{K S} \rightarrow \overline{\mathbf{D C y l}}$ induces an adjunction

$$
j_{!}: \operatorname{Alg}_{\mathbf{K S}}(\mathcal{M}) \rightleftarrows \operatorname{Alg}_{\overline{\mathbf{D C y l}}}(\mathcal{M}): j^{*}
$$

Since $j$ ! is fully faithful, it is enough to prove that the essential image of $j_{\text {! }}$ is $\operatorname{Alg}_{\frac{\mathbf{D C y l}}{\mathbf{D}}}(\mathcal{M})$. Let $X=(A, B, M): \overline{\mathbf{D C y l}} \rightarrow \mathcal{M}^{\otimes}$ be an object of $\operatorname{Alg}_{\overline{\mathbf{D C y l}}}(\mathcal{M})$ whose evaluations at $D, C$ and $C_{M}$ are $A, B$ and $M$, respectively. Note that the forgetful functor $\operatorname{Alg}_{\overline{\mathbf{D C y l}}}(\mathcal{M}) \rightarrow \mathcal{M} \times \mathcal{M} \times \mathcal{M}$ induced by evaluations at $D, C$, and $C_{M}$ is conservative. Thus if we write $q: \mathcal{M}^{\otimes} \rightarrow \Gamma$ for the structure map, $X$ belongs to $\operatorname{Alg} \frac{\mathbf{D}}{\mathbf{D C y l}}(\mathcal{M})$ if and only if

$$
p^{\prime}: \quad K^{\triangleright}:=\left(\mathbf{E}_{2}^{\otimes} \times{ }_{\mathbf{D C y l}} \mathbf{D C y l}_{/ C}^{\text {act }}\right)^{\triangleright} \rightarrow \overline{\mathbf{D C y l}} \rightarrow \mathcal{M}^{\otimes}
$$

is an operadic $q$-colimit diagram that extends $p=\left.p^{\prime}\right|_{K}$, see [25, Definition 3.1.2.1]. Let $Y: \mathbf{K S} \rightarrow$ $\mathcal{M}^{\otimes}$ be an object of $\operatorname{Alg}_{\mathbf{K S}}(\mathcal{M})$. Let us consider the image of $C$ under $j_{!}(Y): \overline{\mathbf{D C y l}} \rightarrow \mathcal{M}^{\otimes}$. It is an operadic $q$-colimit of

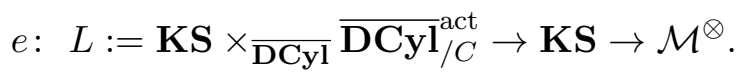

In view of [25, Propositions 3.1.1.15 and 3.1.1.16], to prove the image of $j$ ! is contained in $\operatorname{Alg} \frac{\mathbf{D}}{\overline{\mathbf{D C y l}}}(\mathcal{M})$, it is enough to observe that the natural functor $\mathbf{E}_{2}^{\otimes} \times{ }_{\mathbf{D C y l}} \mathbf{D C} \mathbf{C y l}_{/ C}^{\text {act }} \rightarrow \mathbf{K S} \times \overline{\mathbf{D C y l}}$ $\overline{\mathbf{D C y l}}_{/ C}^{\text {act }}$ is a categorical equivalence. It follows from the fact that if a sequence $E=(D, \ldots, D$, $C, \ldots, C, C_{M}, \ldots C_{M}$ ) (regarded as an object of $\overline{\mathbf{D C y l}}$ ) contains $C_{M}$, then there is no morphism from $E$ to $C$ in $\overline{\mathbf{D C y l}}^{\text {act }}$. Consequently, we have a new adjunction $j_{!}: \operatorname{Alg}_{\mathbf{K S}}(\mathcal{M}) \rightleftarrows$ $\operatorname{Alg} \frac{\mathbf{D}}{\mathbf{D C y} \mathbf{l}}(\mathcal{M}): j^{*}$. By the comparison of operadic $q$-colimit diagrams from $K^{\triangleright}$ and $L^{\triangleright}$, the counit map of this adjunction is an equivalence. Therefore, we obtain a categorical equivalence $\operatorname{Alg}_{\mathbf{K S}}(\mathcal{M}) \simeq \operatorname{Alg} \frac{\mathbf{D}}{\mathbf{D C y l}}(\mathcal{M})$ induced by $j !\left(\right.$ or $\left.j^{*}\right)$.

\section{$5 \quad$ Hochschild cohomology}

In this section, we recall Hochschild cohomology spectra of stable $\infty$-categories $\mathcal{C}$. The definition is based on the principle that, under a suitable condition on $\mathcal{C}$, Hochschild cohomology of $\mathcal{C}$ is the endomorphism algebra of the identity functor $\mathcal{C} \rightarrow \mathcal{C}$. Moreover, $\operatorname{since} \operatorname{Fun}(\mathcal{C}, \mathcal{C})$ has the monoidal structure given by the composition, Hochschild cohomology is the endomorphism algebra of the unit object of $\operatorname{Fun}(\mathcal{C}, \mathcal{C})$ so that it comes equipped with the structure of an $\mathbf{E}_{2}$-algebra, cf. [4, 22] (see also references cited in loc. cit. for Deligne conjecture concerning Hochschild cochains). We establish some notation. Let $R$ be a commutative ring spectrum. Let $\operatorname{Mod}_{R}^{\otimes}$ be the symmetric monoidal $\infty$-category of $R$-module spectra whose underlying category we denote by $\operatorname{Mod}_{R}$. Let $\operatorname{Alg}_{\text {As }}\left(\operatorname{Mod}_{R}\right)$ be the $\infty$-category of the associative algebra objects in $\operatorname{Mod}_{R}$. Let $\operatorname{Pr}^{\mathrm{L}}$ be the $\infty$-category of presentable $\infty$-categories whose morphisms are those functors that preserve small colimits. This category $\operatorname{Pr}^{\mathrm{L}}$ admits a symmetric monoidal structure, see [25, Notation 4.8.1.7 and Proposition 4.8.1.15]. The $\infty$-category of small spaces $\mathcal{S}$ is a unit object in $\operatorname{Pr}^{\mathrm{L}}$. For $\mathcal{D}, \mathcal{D}^{\prime} \in \operatorname{Pr}^{\mathrm{L}}$, the tensor product $\mathcal{D} \otimes \mathcal{D}^{\prime}$ comes equipped with a functor $\mathcal{D} \times \mathcal{D}^{\prime} \rightarrow \mathcal{D} \otimes \mathcal{D}^{\prime}$ which preserves small colimits separately in each variable and satisfies the following universal property: for any $\mathcal{F} \in \operatorname{Pr}^{\mathrm{L}}$, the composition induces a fully faithful functor

$$
\operatorname{Fun}^{\mathrm{L}}\left(\mathcal{D} \otimes \mathcal{D}^{\prime}, \mathcal{F}\right) \rightarrow \operatorname{Fun}\left(\mathcal{D} \times \mathcal{D}^{\prime}, \mathcal{F}\right)
$$

whose essential image is spanned by those functors $\mathcal{D} \times \mathcal{D}^{\prime} \rightarrow \mathcal{F}$ which preserves small colimits separately in each variable, where $\operatorname{Fun}^{\mathrm{L}}(-,-)$ indicates the full subcategory of $\operatorname{Fun}(-,-)$ spanned by those functors which preserves small colimits. The underlying associative monoidal 
$\infty$-category $\operatorname{Mod}_{R}^{\otimes}$ can be regarded as an associative algebra object in $\operatorname{Pr}^{\mathrm{L}}$ since $\operatorname{Mod}_{R}$ is presentable and the tensor product functor $\operatorname{Mod}_{R} \times \operatorname{Mod}_{R} \rightarrow \operatorname{Mod}_{R}$ preserves small colimits separately in each variable. We denote by $\operatorname{LMod}_{\operatorname{Mod}_{R}^{\otimes}}\left(\operatorname{Pr}^{L}\right)$ the $\infty$-category of left $\operatorname{Mod}_{R^{-}}^{\otimes}$ module objects in $\operatorname{Pr}^{\mathrm{L}}$. Given $A \in \operatorname{Alg}_{\text {As }}\left(\operatorname{Mod}_{R}\right)$, we write $\operatorname{RMod}_{A}:=\operatorname{RMod}_{A}\left(\operatorname{Mod}_{R}\right)$ for the $\infty$-category of right module objects of $A$ in $\operatorname{Mod}_{R}$. We remark that the forgetful lax symmetric monoidal functor $\operatorname{Mod}_{R} \rightarrow$ Sp induces $\operatorname{Mod}_{A}\left(\operatorname{Mod}_{R}\right) \rightarrow \operatorname{Mod}_{A}(\mathrm{Sp})$, where we use the same notation $A$ to indicate the image in $\operatorname{Alg}_{\mathrm{As}}(\mathrm{Sp})$. The functor $\operatorname{Mod}_{A}\left(\operatorname{Mod}_{R}\right) \rightarrow \operatorname{Mod}_{A}(\mathrm{Sp})$ is an equivalence of $\infty$-categories (for example, apply Lurie-Barr-Beck theorem to this functor endowed with projections to $\mathrm{Sp}$ ) so that the notation $\mathrm{RMod}_{A}$ is consistent with that of [25]. The category $\operatorname{RMod}_{A}$ has a natural left module structure $\operatorname{Mod}_{R} \times \operatorname{RMod}_{A} \rightarrow \operatorname{RMod}_{A}$ informally given by $(M, N) \mapsto M \otimes_{R} N$. In what follows, when we treat the tensor product of objects in $\operatorname{Mod}_{R}\left(\right.$ over $R$ ), we write $\otimes$ for $\otimes_{R}$. The assignment $A \mapsto \operatorname{RMod}_{A}$ gives rise to a functor

$$
\operatorname{Alg}_{\mathrm{As}}\left(\operatorname{Mod}_{R}\right) \rightarrow \operatorname{LMod}_{\operatorname{Mod}_{R}}\left(\operatorname{Pr}^{\mathrm{L}}\right)
$$

that sends $A$ to $\operatorname{RMod}_{A}$ and carries a morphism $f: A \rightarrow B$ to the base change functor $\operatorname{RMod}_{A} \rightarrow$ $\operatorname{RMod}_{B} ; N \mapsto N \otimes_{A} B$, that is, a left adjoint of the forgetful functor $\operatorname{RMod}_{B} \rightarrow \operatorname{RMod}_{A}$, see [25, Section 4.8.3, Notation 4.8.5.10 and Theorem 4.8.5.11]. We have the induced functor

$$
\text { I: } \quad \operatorname{Alg}_{\mathrm{As}_{\mathrm{s}}}\left(\operatorname{Mod}_{R}\right) \simeq \operatorname{Alg}_{\mathrm{As}}\left(\operatorname{Mod}_{R}\right)_{R /} \rightarrow \operatorname{LMod}_{\operatorname{Mod}_{R}^{\otimes}}\left(\operatorname{Pr}^{\mathrm{L}}\right)_{\operatorname{Mod}_{R} /},
$$

which sends $A$ to the base change functor $\operatorname{Mod}_{R}=\operatorname{RMod}_{R} \rightarrow \operatorname{RMod}_{A}$. The functor $I$ is fully faithful and admits a right adjoint $E$. A morphism $f: \operatorname{Mod}_{R} \rightarrow \mathcal{D}$ in $\operatorname{LMod}_{\operatorname{Mod}_{R}^{\otimes}}\left(\operatorname{Pr}^{L}\right)$ is determined by the image $f(R)$ of $R \in \operatorname{Mod}_{R}$ in an essentially unique way (up to a contractible space of choices). Therefore, an object of $\operatorname{LMod}_{\operatorname{Mod}_{R}^{\otimes}}\left(\operatorname{Pr}^{L}\right)_{\operatorname{Mod}_{R} /}$ is regarded as a pair $(\mathcal{D}, D)$ such that $\mathcal{D}$ belongs to $\operatorname{LMod}_{\operatorname{Mod}_{R}^{\otimes}}\left(\operatorname{Pr}^{\mathrm{L}}\right)$ and $D$ is an object of $\mathcal{D}$. The essential image of $I$ can naturally be identified with $A \lg _{\mathrm{As}}\left(\operatorname{Mod}_{R}\right)$. Namely, it consists of pairs of the form $\left(\operatorname{RMod}_{A}, A\right)$ : $I$ carries $A$ to $\left(\operatorname{RMod}_{A}, A\right)$. Put another way, the essential image is spanned by pairs $(\mathcal{D}, D)$ such that $\mathcal{D}$ is a compactly generated stable $\infty$-category equipped with a single compact generator $D$. The right adjoint $E$ sends $(\mathcal{D}, D)$ to an endormorphism algebra object $\operatorname{End}(D) \in \operatorname{Alg}_{\mathrm{As}}\left(\operatorname{Mod}_{R}\right)$ [25, Theorem 4.8.5.11]. Since the left adjoint $I$ is fully faithful, the unit map id $\rightarrow E \circ I$ is a natural equivalence. Namely, the adjunction $(I, E)$ is a colocalization. If we denote $\mathcal{A} \subset$ $\operatorname{LMod}_{\operatorname{Mod}_{R}^{\otimes}}\left(\operatorname{Pr}^{\mathrm{L}}\right)_{\operatorname{Mod}_{R} /}$ by the essential image of $I$, then $E$ induces a categorical equivalence $\mathcal{A} \stackrel{\sim}{\rightarrow} \operatorname{Alg}_{\mathrm{As}}\left(\operatorname{Mod}_{R}\right)$.

The functor $I$ is extended to a symmetric monoidal functor. To explain this, note that $\mathrm{Alg}_{\mathrm{As}}\left(\operatorname{Mod}_{R}\right)$ comes equipped with a symmetric monoidal structure induced by that of $\operatorname{Mod}_{R}^{\otimes}$, see [25, Section 3.2.4] or Construction 7.9. Since $\operatorname{Mod}_{R}^{\otimes}$ is a symmetric monoidal $\infty$-category such that $\operatorname{Mod}_{R}$ has small colimits and the tensor product functor $\operatorname{Mod}_{R} \times \operatorname{Mod}_{R} \rightarrow \operatorname{Mod}_{R}$ preserves small colimits separately in each variable, we define $\operatorname{Mod}_{\operatorname{Mod}_{R}^{\otimes}}^{\otimes}\left(\operatorname{Pr}^{L}\right)$ to be the symmetric monoidal $\infty$-category of $\operatorname{Mod}_{R}^{\otimes}$-module objects in $\operatorname{Pr}^{\mathrm{L}}$, cf. [25, Section 3.3.3]. If we denote the underlying $\infty$-category by $\operatorname{Mod}_{\operatorname{Mod}_{R}^{\otimes}}\left(\operatorname{Pr}^{L}\right)$, then $\operatorname{Mod}_{\operatorname{Mod}_{R}^{\otimes}}\left(\operatorname{Pr}^{\mathrm{L}}\right) \simeq \operatorname{LMod}_{\operatorname{Mod}_{R}^{\otimes}}\left(\operatorname{Pr}^{\mathrm{L}}\right)$. Hence $\operatorname{LMod}_{\operatorname{Mod}_{R}^{\otimes}}\left(\operatorname{Pr}^{\mathrm{L}}\right)_{\operatorname{Mod}_{R} /} \simeq \operatorname{Mod}_{\operatorname{Mod}_{R}^{\otimes}}\left(\operatorname{Pr}^{L}\right)_{\operatorname{Mod}_{R} /} \simeq \operatorname{Alg}_{\mathbf{E}_{0}^{\otimes}}\left(\operatorname{Mod}_{\operatorname{Mod}_{R}^{\otimes}}\left(\operatorname{Pr}^{L}\right)\right)$ inherits a symmetric monoidal structure. In summary, we have the adjunction

$$
\text { I: } \quad \operatorname{Alg}_{\mathrm{As}_{\mathrm{s}}}\left(\operatorname{Mod}_{R}\right) \rightleftarrows \operatorname{LMod}_{\operatorname{Mod}_{R}^{\otimes}}\left(\operatorname{Pr}^{\mathrm{L}}\right)_{\operatorname{Mod}_{R} /}: E
$$

whose left adjoint is symmetric monoidal and fully faithful, and whose right adjoint is lax symmetric monoidal. It gives rise to an adjunction

$$
\begin{aligned}
I: \operatorname{Alg}_{\mathrm{As}}\left(\operatorname{Alg}_{\mathrm{As}}\left(\operatorname{Mod}_{R}\right)\right) & \rightleftarrows \operatorname{Alg}_{\mathrm{As}}\left(\operatorname{Mod}_{\operatorname{Mod}_{R}^{\otimes}}\left(\operatorname{Pr}^{\mathrm{L}}\right)_{\operatorname{Mod}_{R} /}\right) \\
& \simeq \operatorname{Alg}_{\mathrm{As}}\left(\operatorname{Mod}_{\operatorname{Mod}_{R}^{\otimes}}\left(\operatorname{Pr}^{L}\right)\right): E,
\end{aligned}
$$


where we abuse notation by writing $(I, E)$ for the induced adjunction. By virtue of the canonical equivalence $\mathbf{E}_{1}^{\otimes} \simeq \mathrm{As}^{\otimes}$ and the $\infty$-operad version of Dunn additivity theorem [25, Theorem 5.1.2.2], we have a canonical equivalence

$$
\operatorname{Alg}_{\mathbf{E}_{2}}\left(\operatorname{Mod}_{R}\right) \simeq \operatorname{Alg}_{\mathrm{As}}\left(\operatorname{Alg}_{\mathrm{As}}\left(\operatorname{Mod}_{R}\right)\right)
$$

(we can also use additivity theorem to the equivalence on the right-hand side).

We refer to an object of $\operatorname{Pr}_{R}^{\mathrm{L}}:=\operatorname{Mod}_{\operatorname{Mod}_{R}^{\otimes}}\left(\operatorname{Pr}^{\mathrm{L}}\right)$ as an $R$-linear presentable $\infty$-category. Note that the underlying $\infty$-category of an $R$-linear presentable $\infty$-cartegory is stable.

Lemma 5.1. Let $\mathcal{D}$ be an $R$-linear presentable $\infty$-category. Let $\otimes_{R}: \operatorname{Pr}_{R}^{\mathrm{L}} \times \operatorname{Pr}_{R}^{\mathrm{L}} \rightarrow \operatorname{Pr}_{R}^{\mathrm{L}}$ be the tensor product functor. There exists a morphism object from $\mathcal{D}$ to itself (i.e., an internal hom object) $\mathcal{M o r}_{R}(\mathcal{D}, \mathcal{D}) \in \operatorname{Pr}_{R}^{\mathrm{L}}$ equipped with e: $\mathcal{M o r}_{R}(\mathcal{D}, \mathcal{D}) \otimes_{R} \mathcal{D} \rightarrow \mathcal{D}$ for $\mathcal{D}$. Moreover, $\left(\mathcal{M o r}_{R}(\mathcal{D}, \mathcal{D}), e\right)$ is promoted to an object of $\mathcal{E} \in \operatorname{Alg}_{\mathrm{As}}\left(\operatorname{Pr}_{R}^{\mathrm{L}}\right)$ together with a left module action $\mathcal{E} \otimes_{R} \mathcal{D} \rightarrow \mathcal{D}$.

Proof. According to [25, Corollaries 4.7.1.40 and 4.7.1.41], the second assertion follows from the first assertion. We will show the existence of a morphism object $\operatorname{Mor}_{R}(\mathcal{D}, \mathcal{D})$. Recall that a morphism object for $\mathcal{D}$ and $\mathcal{D}^{\prime}$ is an $R$-linear presentable $\infty$-category $\mathcal{C}$ together with a morphism $\mathcal{C} \otimes_{R} \mathcal{D} \rightarrow \mathcal{D}^{\prime}$ such that the composition induces an equivalence

$$
\operatorname{Map}_{\operatorname{Pr}_{R}^{\mathrm{L}}}(\mathcal{F}, \mathcal{C}) \simeq \operatorname{Map}_{\operatorname{Pr}_{R}^{\mathrm{L}}}\left(\mathcal{F} \otimes_{R} \mathcal{D}, \mathcal{D}^{\prime}\right)
$$

for each $\mathcal{F} \in \operatorname{Pr}_{R}^{\mathrm{L}}$, which informally carries $\mathcal{F} \rightarrow \mathcal{C}$ to $\mathcal{F} \otimes_{R} \mathcal{D} \rightarrow \mathcal{C} \otimes_{R} \mathcal{D} \rightarrow \mathcal{D}$. We first consider the case where $R$ is the sphere spectrum $\mathbb{S}$. Let $\operatorname{Fun}^{\mathrm{L}}\left(\mathcal{D}, \mathcal{D}^{\prime}\right)$ be the full subcategory of $\operatorname{Fun}\left(\mathcal{D}, \mathcal{D}^{\prime}\right)$ that consists of colimit-preserving functors. Then $\operatorname{Fun}^{\mathrm{L}}\left(\mathcal{D}, \mathcal{D}^{\prime}\right)$ together with the evaluation functor $\operatorname{Fun}^{\mathrm{L}}\left(\mathcal{D}, \mathcal{D}^{\prime}\right) \times \mathcal{D} \rightarrow \mathcal{D}^{\prime}$ exhibits $\operatorname{Fun}^{\mathrm{L}}\left(\mathcal{D}, \mathcal{D}^{\prime}\right)$ as an internal hom object. Thus we have a morphism object $\operatorname{Fun}^{\mathrm{L}}\left(\mathcal{D}, \mathcal{D}^{\prime}\right)$. Next, we consider the general case. Let $F$ : $\operatorname{Pr}^{\mathrm{L}} \rightleftarrows$ $\operatorname{Mod}_{\operatorname{Mod}_{R}^{\otimes}}\left(\operatorname{Pr}^{L}\right): U$ be an adjunction which consists of the forgetful functor $U$ and the free functor $F$ given informally by $\mathcal{C} \mapsto \mathcal{C} \otimes \operatorname{Mod}_{R}$. Here $\otimes$ indicates the tensor product in $\operatorname{Pr}^{\mathrm{L}}$. If we suppose that $\mathcal{D}$ is a free object, i.e. $\mathcal{D}=F(\mathcal{C})=\mathcal{C} \otimes \operatorname{Mod}_{R}$, then there is a morphism object for $\mathcal{D}$ and $\mathcal{D}^{\prime}$. Indeed, we observe that $\mathcal{M o r}_{R}\left(\mathcal{D}, \mathcal{D}^{\prime}\right)=\operatorname{Fun}{ }^{\mathrm{L}}\left(\mathcal{C}, \mathcal{D}^{\prime}\right)$ together with

$$
\operatorname{Fun}^{\mathrm{L}}\left(\mathcal{C}, \mathcal{D}^{\prime}\right) \otimes_{R}\left(\mathcal{C} \otimes \operatorname{Mod}_{R}\right) \simeq \operatorname{Fun}^{\mathrm{L}}\left(\mathcal{C}, \mathcal{D}^{\prime}\right) \otimes \mathcal{C} \rightarrow \mathcal{D}^{\prime}
$$

constitutes a morphism object for $\mathcal{D}$ and $\mathcal{D}^{\prime}$. To prove that

$$
\theta: \operatorname{Map}_{\operatorname{Pr}_{R}^{\mathrm{L}}}\left(\mathcal{P}, \operatorname{Fun}^{\mathrm{L}}\left(\mathcal{C}, \mathcal{D}^{\prime}\right)\right) \rightarrow \operatorname{Map}_{\operatorname{Pr}_{R}^{\mathrm{L}}}\left(\mathcal{P} \otimes_{R}\left(\mathcal{C} \otimes \operatorname{Mod}_{R}\right), \mathcal{D}^{\prime}\right)
$$

is an equivalence, we may and will assume that $\mathcal{P}$ is a free object since the tensor operation functor $\otimes_{R}$ preserves small colimits separately in each variable (see the proof of [25, Proposition 5.1.2.9]), and $\mathcal{P}$ is a (small) colimit of the diagram of free objects: for example, using the adjunction $(F, U)$ we have a simplicial diagram of free objects whose colimit is $\mathcal{P}$. When $\mathcal{P}=\mathcal{C}^{\prime} \otimes \operatorname{Mod}_{R}$, by the adjunction we see that $\theta$ is an equivalence. We put $\mathcal{D}=\operatorname{colim}_{i \in I} \mathcal{D}_{i}$, where each $\mathcal{D}_{i}$ is a free object. Then for any $\mathcal{P} \in \operatorname{Pr}_{R}^{\mathrm{L}}$ there exist natural equivalences

$$
\begin{aligned}
\operatorname{Map}_{\operatorname{Pr}_{R}^{\mathrm{L}}}\left(\mathcal{P} \otimes_{R}\left(\operatorname{colim}_{i \in I} \mathcal{D}_{i}\right), \mathcal{D}^{\prime}\right) & \simeq \operatorname{Map}_{\operatorname{Pr}_{R}^{\mathrm{L}}}\left(\operatorname{colim}_{i \in I}\left(\mathcal{P} \otimes_{R} \mathcal{D}_{i}\right), \mathcal{D}^{\prime}\right) \\
& \simeq \lim _{i \in I} \operatorname{Map}_{\operatorname{Pr}_{R}^{\mathrm{L}}}\left(\mathcal{P} \otimes_{R} \mathcal{D}_{i}, \mathcal{D}^{\prime}\right) \\
& \simeq \lim _{i \in I} \operatorname{Map}_{\operatorname{Pr}_{R}^{\mathrm{L}}}\left(\mathcal{P}, \mathcal{M o r}_{R}\left(\mathcal{D}_{i}, \mathcal{D}^{\prime}\right)\right) \\
& \simeq \operatorname{Map}_{\operatorname{Pr}_{R}^{\mathrm{L}}}\left(\mathcal{P}, \lim _{i \in I} \mathcal{M} \operatorname{Mor}_{R}\left(\mathcal{D}_{i}, \mathcal{D}^{\prime}\right)\right)
\end{aligned}
$$

Hence there exists a morphism object $\mathcal{M o r}_{R}\left(\mathcal{D}, \mathcal{D}^{\prime}\right)$, that is, $\lim _{i \in I} \mathcal{M o r}_{R}\left(\mathcal{D}_{i}, \mathcal{D}^{\prime}\right)$. 
Remark 5.2. Let $\mathcal{M o r}_{R}\left(\mathcal{D}, \mathcal{D}^{\prime}\right) \simeq$ be the largest Kan subcomplex of the underlying $\infty$-category of $\mathcal{M o r}_{R}\left(\mathcal{D}, \mathcal{D}^{\prime}\right)$, which we regarded as an object in $\mathcal{S}$. By the above proof, $\mathcal{M o r}_{R}\left(\mathcal{D}, \mathcal{D}^{\prime}\right) \simeq$ is equivalent to the mapping space $\operatorname{Map}_{\operatorname{Pr}_{R}^{\mathrm{L}}}\left(\mathcal{D}, \mathcal{D}^{\prime}\right)$.

We shall write $\mathcal{E} \operatorname{nd}_{R}(\mathcal{D})$ for $\mathcal{E} \in \operatorname{Alg}_{\text {As }}\left(\operatorname{Pr}_{R}^{\mathrm{L}}\right)$.

Definition 5.3. Let $\mathcal{D}$ be an $R$-linear presentable $\infty$-category. Applying $E$ : $\operatorname{Alg}_{\mathrm{As}}\left(\operatorname{Pr}_{R}^{\mathrm{L}}\right) \rightarrow$ $\operatorname{Alg}_{\mathbf{E}_{2}}\left(\operatorname{Mod}_{R}\right)$, we define the Hochschild cohomology $R$-module spectrum of $\mathcal{D}$ to be

$$
\mathcal{H} \mathcal{H}_{R}^{\bullet}(\mathcal{D}):=E\left(\mathcal{E} \operatorname{nd}_{R}(\mathcal{D})\right)
$$

in $\operatorname{Alg}_{\mathbf{E}_{2}}\left(\operatorname{Mod}_{R}\right)$. We often abuse notation by identifying $\mathcal{H} \mathcal{H}_{R}^{\bullet}(\mathcal{D})$ with its image in $\operatorname{Mod}_{R}$. If no confusion can arise, we write $\mathcal{H H}^{\bullet}(\mathcal{D})$ for $\mathcal{H H}_{R}^{\bullet}(\mathcal{D})$.

Let $\mathcal{S}$ t be the $\infty$-category of small stable idempotent-complete $\infty$-categories whose morphisms are exact functors. Let $\mathcal{C}$ be a small stable idempotent-complete $\infty$-category and let $\operatorname{Ind}(\mathcal{C})$ denote the $\infty$-category of Ind-objects. Then $\operatorname{Ind}(\mathcal{C})$ is a compactly generated stable $\infty$-category. The inclusion $\mathcal{C} \rightarrow \operatorname{Ind}(\mathcal{C})$ identifies the essential image with the full subcategory $\operatorname{Ind}(\mathcal{C})^{\omega}$ spanned by compact obejcts in $\operatorname{Ind}(\mathcal{C})$. Given $\mathcal{C}, \mathcal{C}^{\prime} \in \mathcal{S}$ t, if we write $\operatorname{Fun}^{\text {ex }}\left(\mathcal{C}, \mathcal{C}^{\prime}\right)$ for the full subcategory spanned by exact functors, the left Kan extension [27, Proposition 5.3.5.10] gives rise to a fully faithful functor $\operatorname{Fun}^{\mathrm{ex}}\left(\mathcal{C}, \mathcal{C}^{\prime}\right) \rightarrow \operatorname{Fun}^{\mathrm{L}}\left(\operatorname{Ind}(\mathcal{C}), \operatorname{Ind}\left(\mathcal{C}^{\prime}\right)\right)$ whose essential image consists of those functors that carry $\mathcal{C}$ to $\mathcal{C}^{\prime}$. We set $\operatorname{Pr}_{\mathrm{St}}^{\mathrm{L}}=\operatorname{Mod}_{\mathrm{Sp}} \otimes\left(\operatorname{Pr}^{\mathrm{L}}\right)$, which can be regarded as the full subcategory of $\mathrm{Pr}^{\mathrm{L}}$ that consists of stable presentable $\infty$-categories. The assignment $\mathcal{C} \mapsto \operatorname{Ind}(\mathcal{C})$ identifies $\mathcal{S}$ t with the subcategory of $\operatorname{Pr}_{\text {St }}^{\mathrm{L}}$ whose objects are compactly generated stable $\infty$-categories, and whose morphisms are those functors that preserve compact objects. The $\infty$-category $\mathcal{S}$ t inherits a symmetric monoidal structure from the structure on $\operatorname{Pr}_{\mathrm{St}}^{\mathrm{L}}$. The stable $\infty$-category of compact spectra is a unit object in $\mathcal{S}$. Given two objects $\mathcal{C}$ and $\mathcal{C}^{\prime}$ of $\mathcal{S}$ t, the tensor product $\mathcal{C} \otimes \mathcal{C}^{\prime}$ is naturally equivalent to the full subcategory $\left(\operatorname{Ind}(\mathcal{C}) \otimes \operatorname{Ind}\left(\mathcal{C}^{\prime}\right)\right)^{\omega} \subset \operatorname{Ind}(\mathcal{C}) \otimes \operatorname{Ind}\left(\mathcal{C}^{\prime}\right)$ spanned by compact objects. If we let $\operatorname{Cgt}_{\mathrm{St}}^{\mathrm{L}}$ denote the full subcategory of $\operatorname{Pr}_{\mathrm{St}}^{\mathrm{L}}$ spanned by compactly generated stable $\infty$-categories, then we have a sequence

$$
\mathcal{S t} \rightarrow \mathrm{Cgt}_{\mathrm{St}}^{\mathrm{L}} \subset \operatorname{Pr}_{\mathrm{St}}^{\mathrm{L}}
$$

where $\operatorname{Cgt}_{\mathrm{St}}^{\mathrm{L}} \subset \operatorname{Pr}_{\mathrm{St}}^{\mathrm{L}}$ is closed under the tensor product so that $\mathrm{Cgt}_{\mathrm{St}}^{\mathrm{L}}$ inherits a symmetric monoidal structure from the structure on $\operatorname{Pr}_{\mathrm{St}}^{\mathrm{L}}$, and the left arrow is a symmetric monoidal faithful functor given by $\mathcal{C} \mapsto \operatorname{Ind}(\mathcal{C})$. In $\mathrm{Cgt}_{\mathrm{St}}^{\mathrm{L}}$, every object is dualizable. For more details, we refer the readers to [6, Section 3], [25, Section 4.8].

Consider $\operatorname{RMod}_{A}$ for $A \in \operatorname{Alg}_{\text {As }}\left(\operatorname{Mod}_{R}\right)$. We let RPerf $A$ be the full subcategory of $\operatorname{RMod}_{A}$ spanned by compact objects. This subcategory is the smallest stable subcategory which contains $A$ (regarded as a right module) and is closed under retracts. When $A$ belongs to $\mathrm{CAlg}\left(\operatorname{Mod}_{R}\right)$, we write $\operatorname{Perf}_{A}$ for RPerf $A_{A}$. In this case, $\operatorname{Perf}_{A}$ is closed under taking tensor product so that it inherits a symmetric monoidal structure from that of $\operatorname{Mod}_{A}^{\otimes}$. We usually regard the symmetric monoidal $\infty$-category $\operatorname{Perf}_{R}^{\otimes}$ as an object of $\operatorname{CAlg}(\mathcal{S t})$, and we write $\mathcal{S} t_{R}$ for $\operatorname{Mod}_{\operatorname{Perf}_{R}^{\otimes}}(\mathcal{S t})$. We refer to an object of $\mathcal{S} \mathrm{t}_{R}$ as a small $R$-linear stable $\infty$-category. Since $\operatorname{Mod}_{R}^{\otimes} \simeq \operatorname{Ind}\left(\operatorname{Perf}_{R}\right)^{\otimes}$, there is a natural symmetric monoidal functor $\mathcal{S} \mathrm{t}_{R} \rightarrow \operatorname{Pr}_{R}^{\mathrm{L}}=\operatorname{Mod}_{\operatorname{Mod}_{R}^{\otimes}}\left(\operatorname{Pr}^{\mathrm{L}}\right)$ which carries $\mathcal{C}$ to $\operatorname{Ind}(\mathcal{C})$.

Definition 5.4. Given $\mathcal{C} \in \mathcal{S} \mathrm{t}_{R}$, we define the Hochschild cohomology $R$-module spectrum $\mathcal{H H}_{R}^{\bullet}(\mathcal{C})$ to be $\mathcal{H H}_{R}^{\bullet}(\operatorname{Ind}(\mathcal{C}))$. If no confusion can arise, we write $\mathcal{H H}^{\bullet}(\mathcal{C})$ for $\mathcal{H H}_{R}^{\bullet}(\mathcal{C})$. 


\section{Hochschild homology}

Let $R$ be a commutative ring spectrum. Suppose that we are given a small $R$-linear stable $\infty$-category $\mathcal{C}$. In this section, we assign to $\mathcal{C} \in \mathcal{S} \mathrm{t}_{R}$ the Hochschild homology $R$-module spectrum $\mathcal{H} \mathcal{H}_{\bullet}(\mathcal{C}) \in \operatorname{Mod}_{R}$. For the main purpose of this paper, we require the following additional structures:

- the $R$-module spectrum $\mathcal{H} \mathcal{H}_{\bullet}(\mathcal{C})$ has an action of the circle $S^{1}$. Namely, $\mathcal{H} \mathcal{H} \bullet(\mathcal{C})$ is promoted to an object of $\operatorname{Fun}\left(B S^{1}, \operatorname{Mod}_{R}\right)$, and the assignment $\mathcal{C} \mapsto \mathcal{H} \mathcal{H}_{\bullet}(\mathcal{C})$ gives rise to a functor $\mathcal{S} \mathrm{t}_{R} \rightarrow \operatorname{Fun}\left(B S^{1}, \operatorname{Mod}_{R}\right)$,

- if $\operatorname{Fun}\left(B S^{1}, \operatorname{Mod}_{R}\right)$ is equipped with a pointwise symmetric monoidal strcuture induced by that of $\operatorname{Mod}_{R}$, then the above functor $\mathcal{S} \mathrm{t}_{R} \rightarrow \operatorname{Fun}\left(B S^{1}, \operatorname{Mod}_{R}\right)$ is promoted to a symmetric monoidal functor from $\mathcal{S} \mathrm{t}_{R}$ to $\operatorname{Fun}\left(B S^{1}, \operatorname{Mod}_{R}\right)$.

To this end, we will use enriched models of stable idempotent-complete $\infty$-categories, i.e., spectral categories.

Symmetric spectra. We give a minimal review of the theory of symmetric spectra, introduced and developed in [17]. This theory provides a nice foundation of the homotopy theory of highly structured ring spectra as well as a theoretical basis for spectral categories. We let $\mathrm{Sp}^{\Sigma}$ be the closed symmetric monoidal category of symmetric spectra. We write $\mathbb{S}$ for the unit object which we call the sphere spectrum. We use the notation slightly different from [17, 32]: $\mathbb{S}$ is $S$ in [17]. We use a symmetric monoidal proper combinatorial model category structure on $\mathrm{Sp}^{\Sigma}$ satisfying the monoid axiom in the sense of [30, Definition 3.3], in which a weak equivalence is a stable equivalence. There are several versions of such model structures. We here focus on two model structures. One is described in [17, Theorems 3.4.4 and 5.4.2, Corollaries 5.3.8 and 5.5.2] which is called the stable model structure. In [32], it is proved that there is another model structure called the stable $\mathbb{S}$-model structure. The difference (relevant to us) between stable model structure and stable $\mathbb{S}$-model structure is that cofibrations in the stable model structure [17, Theorem 3.4.4] are contained in the class of cofibrations in the stable $\mathbb{S}$-model structure while both have the same class of weak equivalences. Let $\operatorname{CAlg}\left(\mathrm{Sp}^{\Sigma}\right)$ denote the category of commutative algebra objects in $\mathrm{Sp}^{\Sigma}$. We refer to an object of CAlg $\left(\mathrm{Sp}^{\Sigma}\right)$ as a commutative symmetric ring spectrum. The category $\mathrm{CAlg}\left(\mathrm{Sp}^{\Sigma}\right)$ admits a model category structure: we use the model structure on CAlg $\left(\mathrm{Sp}^{\Sigma}\right)$, defined in [32, Theorem 3.2] in which a morphism is a weak equivalence if the underlying morphism in $\mathrm{Sp}^{\Sigma}$ is a stable equivalence. The stable $\mathbb{S}$-model structure on $\mathrm{Sp}^{\Sigma}$ has the following pleasant property: if $\mathbb{R}$ is a cofibrant object in $\operatorname{CAlg}\left(\mathrm{Sp}^{\Sigma}\right)$, then the underlying object $\mathbb{R}$ in $\mathrm{Sp}^{\Sigma}$ is cofibrant with respect to the stable $\mathbb{S}$-model structure, see [32, Section 4].

Let $\mathbb{R}$ be a commutative symmetric ring spectrum, which we think of as a model of $R \in$ $\mathrm{CAlg}(\mathrm{Sp})$. Unless otherwise stated, we assume that $\mathbb{R}$ is cofibrant in $\mathrm{CAlg}\left(\mathrm{Sp}^{\Sigma}\right)$. We let $\mathrm{Sp}^{\Sigma}(\mathbb{R})$ denote the category of $\mathbb{R}$-module objects in $\mathrm{Sp}^{\Sigma}$, which is endowed with the natural symmetric monoidal structure induced by the structure on $\mathrm{Sp}^{\Sigma}$. In virtue of [32, Theorem 2.6] (or [30, Theorem 4.1]), there is a combinatorial symmetric monoidal projective model structure on $\operatorname{Sp}^{\Sigma}(\mathbb{R})$ satisfying the monoid axiom, in which a morphism is a weak equivalence (resp. a fibration) if the underlying morphism in $\mathrm{Sp}^{\Sigma}$ is a stable equivalence (resp. a fibration with respect to stable $\mathbb{S}$-model structure). We refer to this model structure as the stable $\mathbb{R}$-model structure.

Definition 6.1. Let $\mathbb{R}$ be a commutative symmetric ring spectrum. An $\mathbb{R}$-spectrum category is a category enriched over $\operatorname{Sp}^{\Sigma}(\mathbb{R})$. More explicitly, a (small) $\mathbb{R}$-spectrum category $\mathcal{A}$ consists of the data: 
- A (small) set of objects,

- An $\mathbb{R}$-module symmetric spectrum $\mathcal{A}(X, Y) \in \mathrm{Sp}^{\Sigma}(\mathbb{R})$ for each ordered pair of objetcs $(X, Y)$,

- The composition law $\mathcal{A}(Y, Z) \wedge_{\mathbb{R}} \mathcal{A}(X, Y) \rightarrow \mathcal{A}(X, Z)$ satisfying the standard associativity axiom,

- $\mathbb{S} \rightarrow \mathcal{A}(X, X)$ for each object $X$ that satisfies the standard unit axiom.

Here $\wedge_{\mathbb{R}}$ denotes the wedge product over $\mathbb{R}$, which defines the tensor product in $\operatorname{Sp}^{\Sigma}(\mathbb{R})$. A functor of $\mathbb{R}$-spectral categories is an enriched functor, that is, a functor as enriched categories. We refer to them as $\mathbb{R}$-spectral functors. We write Cat $t_{\mathbb{R}}$ for the category of $\mathbb{R}$-spectral categories whose morphisms are $\mathbb{R}$-spectral functors. We refer to an $\mathbb{S}$-spectral category (resp. an $\mathbb{S}$-spectral functor) as a spectral category (resp. a spectral functor). We write $\wedge$ for $\wedge_{\mathbb{S}}$.

Thanks to works [6, Corollary 2.4], [28, Theorem 1.1], [33, Theorem 7.25], Cat $\mathbb{R}_{\mathbb{R}}$ admits a combinatorial model structure whose weak equivalences are Dwyer-Kan equivalences (DKequivalences for short). See, e.g., [6, Definition 2.1] for DK-equivalences.

Let us recall the notion of Morita equivalences in the context of spectral categories, see [6, Sections 2 and 4] for an excellent account. Let $\mathcal{A}$ be a small spectral category and let Fun $_{\mathbb{S}}\left(\mathcal{A}^{\mathrm{op}}, \mathrm{Sp}^{\Sigma}\right)$ be the spectral category of spectral functors. There is a combinatorial spectral model structure where the class of weak equivalences (resp. fibrations) are objectwise stable equivalences (resp. objectwise fibrations with respect to the stable $\mathbb{S}$-model structure) [29, Appendix]. The enriched Yoneda embedding $\mathcal{A} \rightarrow \operatorname{Fun}_{\mathbb{S}}\left(\mathcal{A}^{\mathrm{op}}, \mathrm{Sp}^{\Sigma}\right)$ is contained in the full subcategory of cofibrant objects. If we replace $\mathcal{A}$ by a fibrant object in Cats, the embedding lands in the full subcategory Fun $\left(\mathcal{A}^{\mathrm{op}}, \mathrm{Sp}^{\Sigma}\right)^{\mathrm{cf}}$ that consists of cofibrant and fibrant objects. Let $F: \mathcal{A} \rightarrow \mathcal{B}$ be a spectral functor of spectral categories. Then we have a Quillen adjunction

$$
F_{!}: \operatorname{Fun}_{\mathbb{S}}\left(\mathcal{A}^{\mathrm{op}}, \mathrm{Sp}^{\Sigma}\right) \rightleftarrows \operatorname{Fun}_{\mathbb{S}}\left(\mathcal{B}^{\mathrm{op}}, \mathrm{Sp}^{\Sigma}\right): F^{*},
$$

where $F^{*}$ is determined by the composition with $\mathcal{A}^{\mathrm{op}} \rightarrow \mathcal{B}^{\mathrm{op}}$, see [29, Appendix]. Let $\mathcal{D}(\mathcal{A})$ be the homotopy category of $\mathcal{D}^{\Sigma}(\mathcal{A}):=\operatorname{Fun}_{\mathbb{S}}\left(\mathcal{A}^{\mathrm{op}}, \mathrm{Sp}^{\Sigma}\right)^{\mathrm{cf}}$. It constitutes a triangulated category. Let $\mathcal{D}_{\text {pe }}(\mathcal{A})$ be the smallest thick subcategory of $\mathcal{D}(\mathcal{A})$ that contains the image of $\mathcal{A}$ under the Yoneda embedding. The subscript "pe" stands for "perfect". We write $\mathcal{D}_{\mathrm{pe}}^{\Sigma}(\mathcal{A})$ for the full subcategory of Fun $\mathcal{S}_{\mathbb{S}}\left(\mathcal{A}^{\text {op }}, \mathrm{Sp}^{\Sigma}\right)^{\text {cf }}$ spanned by those objects that belong to $\mathcal{D}_{\text {pe }}(\mathcal{A})$. If $F: \mathcal{A} \rightarrow \mathcal{B}$ is a spectral functor, we have the induced (left-derived) functor $\mathbb{L} F_{!}: \mathcal{D}(\mathcal{A}) \rightarrow \mathcal{D}(\mathcal{B})$. Since $\mathbb{L} F$ is an exact functor of triangulated categories, it follows that the restrcition of $\mathbb{L} F$ ! induces $\mathbb{L} F_{!}: \mathcal{D}_{\mathrm{pe}}(\mathcal{A}) \rightarrow \mathcal{D}_{\mathrm{pe}}(\mathcal{B})$.

Definition 6.2. We say that a spectral functor $F: \mathcal{A} \rightarrow \mathcal{B}$ is a Morita equivalence if the induced functor $\mathbb{L} F$ ! $\mathcal{D}_{\text {pe }}(\mathcal{A}) \rightarrow \mathcal{D}_{\text {pe }}(\mathcal{B})$ is an equivalence of categories. When $F: \mathcal{A} \rightarrow \mathcal{B}$ is an $\mathbb{R}$-spectral functor, $F$ is said to be a Morita equivalence if $F$ is a Morita equivalence as a spectral functor.

Let $\mathbb{R}$ be a commutative symmetric ring spectrum. Let us recall the tensor product of $\mathbb{R}$ spectral categories. Suppose that we are given $\mathcal{A}, \mathcal{B} \in$ Cat $_{\mathbb{R}}$. The tensor product $\mathcal{A} \wedge_{\mathbb{R}} \mathcal{B}$ is defined by the following data:

- The set of objects of $\mathcal{A} \wedge_{\mathbb{R}} \mathcal{B}$ is the set of pairs $(A, B)$, where $A$ is an object of $\mathcal{A}$, and $B$ is an object of $\mathcal{B}$,

- $\mathcal{A} \wedge_{\mathbb{R}} \mathcal{B}\left((a, b),\left(a^{\prime}, b^{\prime}\right)\right)=\mathcal{A}\left(a, a^{\prime}\right) \wedge_{\mathbb{R}} \mathcal{B}\left(b, b^{\prime}\right)$ for $(a, b),\left(a^{\prime}, b^{\prime}\right) \in \mathcal{A} \wedge_{\mathbb{R}} \mathcal{B}$.

This tensor product determines a symmetric monoidal structure on $\mathrm{Cat}_{\mathbb{R}}$. A unit object is defined as follows: Let $B \mathbb{R}$ be the spectral category which has a single object $*$ together with 
the morphism ring spectrum $B \mathbb{R}(*, *)=\mathbb{R}$. The composition $\mathbb{R} \wedge \mathbb{R} \rightarrow \mathbb{R}$ and the unit $\mathbb{S} \rightarrow \mathbb{R}$ are determined by the algebra structure on $\mathbb{R}$ in an obvious way. Clearly, $B \mathbb{R}$ is a unit object in $\mathrm{Cat}_{\mathbb{R}}$. Since $\mathbb{R}$ is commutative, we can also think of $B \mathbb{R}$ as a symmetric monoidal spectral category. Namely, it is a commutative algebra object in the symmetric monoidal category Cat . $_{\text {. }}$ Note that an $\mathbb{R}$-spectral category $\mathcal{A}$ is regarded as a $B \mathbb{R}$-module in $\mathrm{Sp}^{\Sigma}$. Namely, there is a canonical equivalence of categories $\operatorname{Cat}_{\mathbb{R}} \stackrel{\sim}{\rightarrow} \operatorname{Mod}_{B \mathbb{R}}\left(\right.$ Cat $\left._{\mathbb{S}}\right)$, where the target is the category of $B \mathbb{R}$-module objects in Cat $_{\mathbb{S}}$.

For technical reasons, we use the notion of pointwise-cofibrant spectral categories, cf. [7, Section 4]. We say that an $\mathbb{R}$-spectral category $\mathcal{A}$ is pointwise-cofibrant if each morphism spectrum $\mathcal{A}(X, Y)$ is cofibrant in $\operatorname{Sp}^{\Sigma}(\mathbb{R})$ with respect to the stable $\mathbb{R}$-model structure. Using the same argument as that in the proof in [7, Proposition 4.1], we have:

\section{Proposition 6.3 ([7]).}

(i) Every $\mathbb{R}$-spectral category is functorially Morita equivalent to a pointwise-cofibrant $\mathbb{R}$-spectral category with the same objects.

(ii) The subcategory of pointwise-cofibrant $\mathbb{R}$-spectral category is closed under the tensor product.

(iii) If $\mathcal{A}$ is a pointwise-cofibrant $\mathbb{R}$-spectral category, the tensor operation $\mathcal{A} \wedge_{\mathbb{R}}(-)$ preserves Morita equivalences and colimits.

(iv) If $\mathcal{A}$ and $\mathcal{B}$ are both pointwise-cofibrant $\mathbb{R}$-spectral categories, then the $\mathcal{A} \wedge_{\mathbb{R}} \mathcal{B}$ computes the derived tensor product.

We denote by $\mathrm{Cat}_{\mathbb{S}}^{\mathrm{pc}}$ the category of small pointwise-cofibrant spectral categories. By Proposition 6.3, Cat $\mathrm{t}_{\mathbb{S}}^{\mathrm{pc}}$ admits a symmetric monoidal structure given by tensor products, and the tensor products preserves Morita equivalences in each variable. Similarly, we denote by Cat $\mathrm{t}_{\mathbb{R}}^{\mathrm{pc}}$ the category of small pointwise-cofibrant $\mathbb{R}$-spectral categories.

Inverting morphisms. We recall the notion of $\infty$-categories obtained from an $\infty$-category endowed with a set of morphisms. We refer the readers to [25, Sections 1.3.4 and 4.1.3] for more details. Let $\mathcal{C}$ be an $\infty$-category. Suppose that we are given a set $S$ of edges (morphisms) (we assume all equivalences are contained in $S$ ). Then there exists an $\infty$-category $\mathcal{C}\left[S^{-1}\right]$ together with $\xi: \mathcal{C} \rightarrow \mathcal{C}\left[S^{-1}\right]$ such that for any $\infty$-category $\mathcal{D}$ the composition induces a fully faithful functor

$$
\operatorname{Map}\left(\mathcal{C}\left[S^{-1}\right], \mathcal{D}\right) \rightarrow \operatorname{Map}(\mathcal{C}, \mathcal{D})
$$

whose essential image consists of those functors $F: \mathcal{C} \rightarrow \mathcal{D}$ which carry edges in $S$ to equivalences in $\mathcal{D}$. We shall refer to $\mathcal{C}\left[S^{-1}\right]$ as the $\infty$-category obtained from $\mathcal{C}$ by inverting $S$. We note that $\mathcal{C}\left[S^{-1}\right]$ is generally not locally small even when $\mathcal{C}$ is so. When $\mathcal{C}$ is an ordinary category, an explicit construction of $\mathcal{C}\left[S^{-1}\right]$ is given by the hammock localization [13]. Let $\mathcal{C}^{\otimes}$ be a symmetric monoidal $\infty$-category. Let $S$ be a set of edges in $\mathcal{C}$ such that all equivalences are contained in $S$. Assume that for any object $C \in \mathcal{C}$ and any morphism $C_{1} \rightarrow C_{2}$ in $S$, the induced morphisms $C \otimes C_{1} \rightarrow C \otimes C_{2}$ and $C_{1} \otimes C \rightarrow C_{2} \otimes C$ belong to $S$. Then there exists a symmetric monoidal $\infty$-category $\mathcal{C}\left[S^{-1}\right]^{\otimes}$ together with a symmetric monoidal functor $\tilde{\xi}: \mathcal{C}^{\otimes} \rightarrow \mathcal{C}\left[S^{-1}\right]^{\otimes}$ whose underlying functor is equivalent to $\xi$. There is a universal property: for any symmetric monoidal $\infty$-category $\mathcal{D}^{\otimes}$ the composition induces a fully faithful functor $\operatorname{Map}^{\otimes}\left(\mathcal{C}\left[S^{-1}\right]^{\otimes}, \mathcal{D}^{\otimes}\right) \rightarrow \operatorname{Map}^{\otimes}\left(\mathcal{C}^{\otimes}, \mathcal{D}^{\otimes}\right)$ whose essential image consists of those functors $F: \mathcal{C}^{\otimes} \rightarrow \mathcal{D}^{\otimes}$ which carry morphisms in $S$ to equivalences in $\mathcal{D}$. Here $\operatorname{Map}^{\otimes}(-,-)$ indicates the space of symmetric monoidal functors. 
Example 6.4. Let $\mathrm{Sp}^{\Sigma}(\mathbb{R})^{c}$ be the full subcategory that consists of cofibrant objects. The tensor product $\wedge_{\mathbb{R}}$ given by the wedge product over $\mathbb{R}$ preserves cofibrant objects. In addition, if $C \in \mathrm{Sp}^{\Sigma}(\mathbb{R})^{c}$ and $f: C_{1} \rightarrow C_{2}$ is a weak equivalence (i.e., stable equivalence), then $C \wedge_{\mathbb{R}} f$ is a weak equivalence. Consequently, we have the symmetric monoidal $\infty$-category $\operatorname{Sp}^{\Sigma}(\mathbb{R})^{c}\left[W^{-1}\right]^{\otimes}$ obtained by inverting weak equivalence. In the case of $\mathbb{R}=\mathbb{S}$, by the characterization of $\mathrm{Sp}^{\otimes}$ [25, Corollary 4.8.2.19], there is a canonical (unique) symmetric monoidal equivalence $\operatorname{Sp}^{\Sigma}(\mathbb{S})^{c}\left[W^{-1}\right]^{\otimes} \simeq \mathrm{Sp}^{\otimes}$. In addition, if we denote $R$ by the image of $\mathbb{R}$ in $\mathrm{CAlg}(\mathrm{Sp})$, then by $\left[25\right.$, Theorem 4.3.3.17] there is a canonical symmetric monoidal equivalence $\operatorname{Sp}^{\Sigma}(\mathbb{R})^{c}\left[W^{-1}\right]^{\otimes}$ $\stackrel{\sim}{\rightarrow} \operatorname{Mod}_{R}^{\otimes}\left(\operatorname{Sp}^{\Sigma}(\mathbb{S})^{c}\left[W^{-1}\right]^{\otimes}\right) \simeq \operatorname{Mod}_{R}^{\otimes}\left(\operatorname{Sp}^{\otimes}\right)=\operatorname{Mod}_{R}^{\otimes}$. Let $\operatorname{Alg}_{\text {As }}\left(\operatorname{Sp}^{\Sigma}(\mathbb{R})^{c}\right)$ be the category of associative algebra objects in $\mathrm{Sp}^{\Sigma}(\mathbb{R})^{c}$, which is endowed with the symmetric monoidal structure induced by that of $\operatorname{Sp}^{\Sigma}(\mathbb{R})^{c}$. Then if $\operatorname{Alg}_{A s}\left(\operatorname{Sp}^{\Sigma}(\mathbb{R})^{c}\right)\left[W^{-1}\right]^{\otimes}$ denotes the associated symmetric monoidal $\infty$-category obtained by inverting weak equivalences, then we have equivalences of symmetric monoidal $\infty$-categories

$$
\operatorname{Alg}_{\mathrm{As}}\left(\operatorname{Sp}^{\Sigma}(\mathbb{R})^{c}\right)\left[W^{-1}\right]^{\otimes} \simeq \operatorname{Alg}_{\mathrm{As}}^{\otimes}\left(\left(\operatorname{Sp}^{\Sigma}(\mathbb{R})^{c}\right)\left[W^{-1}\right]\right) \simeq \operatorname{Alg}_{\mathrm{As}}^{\otimes}\left(\operatorname{Mod}_{R}\right),
$$

where the left equivalence follows from the rectification result [25, Theorem 4.1.8.4]. In particular, given an associative algebra $A \in \operatorname{Alg}_{\text {As }}\left(\operatorname{Mod}_{R}\right)$, there is an associative algebra $\mathbb{A} \in$ $\operatorname{Alg}_{\mathrm{As}}\left(\operatorname{Sp}^{\Sigma}(\mathbb{R})^{c}\right)$ together with an equivalence $\sigma: \mathbb{A} \simeq A$ in $\operatorname{Alg}_{\mathrm{As}}\left(\operatorname{Mod}_{R}\right)$. In this case, we say that $\mathbb{A}$ (together with $\sigma$ ) represents $A$.

Example 6.5. We use the symbol $M$ to indicate the class of Morita equivalences in $\mathrm{Cat}_{\mathbb{S}}^{\mathrm{pc}}$ or $\mathrm{Cat}_{\mathbb{R}}^{\mathrm{pc}}$. By Proposition 6.3, we can invert the class of Morita equivalences $M$ to obtain symmetric monoidal $\infty$-categories $\mathrm{Cat}_{\mathbb{S}}^{\mathrm{pc}}\left[M^{-1}\right]^{\otimes}$ and $\mathrm{Cat}_{\mathbb{R}}^{\mathrm{pc}}\left[M^{-1}\right]^{\otimes}$. Thanks to multiplicative Morita theory [7, Theorem 4.6], there is a canonical equivalence of symmetric monoidal $\infty$-categories

$$
\mathcal{S} \mathrm{t}^{\otimes} \simeq \operatorname{Cat}_{\mathbb{S}}^{\mathrm{pc}}\left[M^{-1}\right]^{\otimes}
$$

Construction 6.6. There is a sequence of symmetric monoidal $\infty$-categories

$$
\mathrm{Cat}_{\mathbb{R}}^{\mathrm{pc}} \rightarrow \mathrm{Cat}_{\mathbb{S}}^{\mathrm{pc}} \rightarrow \mathrm{Cat}_{\mathbb{S}}^{\mathrm{pc}}\left[M^{-1}\right]
$$

such that $\mathrm{Cat}_{\mathbb{R}}^{\mathrm{pc}}$ and $\mathrm{Cat}_{\mathbb{S}}^{\mathrm{pc}}$ are ordinary symmetric monoidal categories, the left arrow is the forgetful lax symmetric monoidal functor, and the right arrow is the canonical symmetric monoidal functor. Consequently, if we write $R$ for the image of $\mathbb{R}$ in $\mathrm{CAlg}(\mathrm{Sp})$, it gives rise to a lax symmetric monoidal functor

$$
\pi: \operatorname{Cat}_{\mathbb{R}}^{\mathrm{pc}} \rightarrow \operatorname{Mod}_{B \mathbb{R}}\left(\operatorname{Cat}_{\mathbb{S}}^{\mathrm{pc}}\left[M^{-1}\right]\right) \simeq \operatorname{Mod}_{\operatorname{Perf}_{R}}(\mathcal{S t}) .
$$

To obtain Cat $\mathrm{Ca}_{\mathbb{R}}^{\mathrm{pc}} \rightarrow \mathrm{Cat}_{\mathbb{S}}^{\mathrm{pc}}$, we need to check that the essential image of Cat $\mathrm{T}_{\mathbb{R}}^{\mathrm{pc}} \rightarrow \mathrm{Cat}_{\mathbb{S}}$ is contained in $\mathrm{Cat}_{\mathbb{S}}^{\mathrm{pc}}$. It is enough to show that the forgetful functor $\operatorname{Sp}^{\Sigma}(\mathbb{R}) \rightarrow \mathrm{Sp}^{\Sigma}(\mathbb{S})=\mathrm{Sp}^{\Sigma}$ carries cofibrant objects to cofibrant objects in $\mathrm{Sp}^{\Sigma}$. For this purpose, we recall that cofibrations in $\mathrm{Sp}^{\Sigma}$ with respect to the stable $\mathbb{S}$-model structure is the smallest weakly saturated class $[27$, Definition A.1.2.2] of morphisms that contains $\{\mathbb{S} \otimes i\}_{i \in \text { Mon }}$, where Mon is the class of monomorphisms of symmetric sequences, and $\mathbb{S} \otimes i$ denotes the morphism of symmetric spectrum induced by $i$, namely, $\mathbb{S} \otimes(-)$ is the left adjoint of the forgetful functor from $\mathrm{Sp}^{\Sigma}$ to the category of symmetric sequences, see [32]. The class of cofibrations in $\operatorname{Sp}^{\Sigma}(\mathbb{R})$ with respect to the stable $\mathbb{R}$-model structure is the smallest weakly saturated class of morphisms containing $\{\mathbb{R} \otimes i=\mathbb{R} \wedge(\mathbb{S} \otimes i)\}_{i \in \text { Mon }}$. Note that we assume that $\mathbb{R}$ is a cofibrant object in CAlg $\left(\mathrm{Sp}^{\Sigma}\right)$ so that the underlying object $\mathbb{R}$ is cofibrant in $\mathrm{Sp}^{\Sigma}$. It follows that the underlying morphisms $\mathbb{R} \otimes i$ in $\mathrm{Sp}^{\Sigma}$ are cofibrations. Since $\mathrm{Sp}^{\Sigma}(\mathbb{R}) \rightarrow \mathrm{Sp}^{\Sigma}$ preserves colimits, $\mathrm{Sp}^{\Sigma}(\mathbb{R}) \rightarrow \mathrm{Sp}^{\Sigma}$ preserves cofibrations. 
The following is a rectification result for $\mathcal{S} \mathrm{t}_{R}^{\otimes}$.

Proposition 6.7. $\pi: \mathrm{Cat}_{\mathbb{R}}^{\mathrm{pc}} \rightarrow \operatorname{Mod}_{B \mathbb{R}}\left(\mathrm{Cat}_{\mathbb{S}}^{\mathrm{pc}}\left[M^{-1}\right]\right)$ in Construction 6.6 induces a symmetric monoidal equivalence

$$
\operatorname{Cat}_{\mathbb{R}}^{\mathrm{pc}}\left[M^{-1}\right]^{\otimes} \simeq \operatorname{Mod}_{B \mathbb{R}}^{\otimes}\left(\operatorname{Cat}_{\mathbb{S}}^{\mathrm{pc}}\left[M^{-1}\right]\right)
$$

In particular, we have a canonical symmetric monoidal equivalence

$$
\mathrm{Cat}_{\mathbb{R}}^{\mathrm{pc}}\left[M^{-1}\right]^{\otimes} \simeq \operatorname{Mod}_{\operatorname{Perf}_{R}}^{\otimes}(\mathcal{S t})=\mathcal{S} \mathrm{t}_{R}^{\otimes} .
$$

Proof. The underlying functor is an equivalence of $\infty$-categories. In fact, there are sequence of categorical equivalences

$$
\mathrm{Cat}_{\mathbb{R}}^{\mathrm{pc}}\left[M^{-1}\right] \rightarrow \operatorname{Mod}_{B \mathbb{R}}\left(\mathrm{Cat}_{\mathbb{S}}^{\mathrm{pc}}\right)\left[M^{-1}\right] \rightarrow \operatorname{Mod}_{B \mathbb{R}}\left(\operatorname{Cat}_{\mathbb{S}}^{\mathrm{pc}}\left[M^{-1}\right]\right),
$$

where the middle $\infty$-category is obtained from the ordinary category $\operatorname{Mod}_{B \mathbb{R}}\left(\mathrm{Cat}_{\mathbb{S}}^{\mathrm{pc}}\right)$ by inverting Morita equivalences (we do not claim that the middle $\infty$-category admits a monoidal structure). The right arrow is induced by $\operatorname{Mod}_{B \mathbb{R}}\left(\operatorname{Cat}_{\mathbb{S}}^{\mathrm{pc}}\right) \rightarrow \operatorname{Mod}_{B \mathbb{R}}\left(\operatorname{Cat}_{\mathbb{S}}^{\mathrm{pc}}\left[M^{-1}\right]\right)$. The left arrow is induced by the inclusion $\mathrm{Cat}_{\mathbb{R}}^{\mathrm{pc}} \hookrightarrow \operatorname{Mod}_{B \mathbb{R}}\left(\operatorname{Cat}_{\mathbb{S}}^{\mathrm{pc}}\right) \subset \operatorname{Mod}_{B \mathbb{R}}\left(\operatorname{Cat}_{\mathbb{S}}\right) \simeq \operatorname{Cat}_{\mathbb{R}}$. The functorial cofibrant resolution $\mathrm{Cat}_{\mathbb{R}} \rightarrow \mathrm{Cat}_{\mathbb{R}}^{\mathrm{pc}}$ induces an inverse equivalence of $\mathrm{Cat}_{\mathbb{R}}^{\mathrm{pc}}\left[M^{-1}\right]$ $\rightarrow \operatorname{Mod}_{B \mathbb{R}}\left(\mathrm{Cat}_{\mathbb{S}}^{\mathrm{pc}}\right)\left[M^{-1}\right]$ (a cofibrant one is pointwise-cofibrant). It is proved in [9, Theorem 5.1] that the right arrow is a categorical equivalence. Next, we prove that $\pi: \mathrm{Cat}_{\mathbb{R}}^{\mathrm{pc}} \rightarrow$ $\operatorname{Mod}_{B \mathbb{R}}\left(\operatorname{Cat}_{\mathbb{S}}^{\mathrm{pc}}\left[M^{-1}\right]\right)$ is symmetric monoidal. Namely, we show that the natural morphisms $\operatorname{Perf}_{R} \rightarrow \pi(B \mathbb{R})$ and $\pi(\mathcal{A}) \otimes_{R} \pi(\mathcal{B}) \rightarrow \pi\left(\mathcal{A} \wedge_{\mathbb{R}} \mathcal{B}\right)$ are equivalences in $\operatorname{Mod}_{B \mathbb{R}}\left(\operatorname{Cat}_{\mathbb{S}}^{\mathrm{pc}}\left[M^{-1}\right]\right) \simeq$ $\operatorname{Mod}_{\operatorname{Perf}_{R}}(\mathcal{S t})$, where $\pi(\mathcal{A}) \otimes_{R} \pi(\mathcal{B})$ indicates the tensor product in $\operatorname{Mod}_{\operatorname{Perf}_{R}}(\mathcal{S} \mathrm{t})$. By construction, the morphism $\operatorname{Perf}_{R} \rightarrow \pi(B \mathbb{R})$ is an equivalence. We prove that $\pi(\mathcal{A}) \otimes_{R} \pi(\mathcal{B}) \rightarrow \pi\left(\mathcal{A} \wedge_{\mathbb{R}} \mathcal{B}\right)$ is an equivalence. We here write $\widehat{\mathcal{A}}_{\text {pe }}$ for the $\mathbb{R}$-spectral full subcategory of $\operatorname{Fun}_{\mathbb{R}}\left(\mathcal{A}^{\text {op }}, \operatorname{Sp}^{\Sigma}(\mathbb{R})\right)^{\text {cf }}$ that consists of cofibrant-fibrant objects lying over $\mathcal{D}_{\mathrm{pe}}(\mathcal{A})_{\mathbb{R}}\left(\widehat{\mathcal{A}}_{\mathrm{pe}}\right.$ is DK-equivalent to $\mathcal{D}_{\mathrm{pe}}^{\Sigma}(\mathcal{A})$ as spectral categories, see the proof of Lemma 6.11 and Claim 6.12 for the notation). For any other $\mathbb{R}$-spectral category $\mathcal{P}$, we define $\widehat{\mathcal{P}}_{\text {pe }}$ in the same way. By Claim 6.12 below, the image of $\left(\widehat{\mathcal{A} \wedge_{\mathbb{R}}} \mathcal{B}\right)_{\text {pe }}$ in $\operatorname{Mod}_{B \mathbb{R}}\left(\operatorname{Cat}_{\mathbb{S}}^{\mathrm{pc}}\left[M^{-1}\right]\right)$ is equivalent to $\pi\left(\mathcal{A} \wedge_{\mathbb{R}} \mathcal{B}\right)$ in the natural way. Let $\left\{\mathcal{A}_{\lambda}\right\}_{\lambda \in \Lambda}$ be the filtered family (poset) of $\mathbb{R}$-spectral full subcategories of $\mathcal{A}$ such that for any $\mathcal{A}_{\lambda}$,

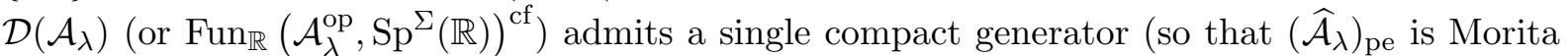
equivalent to $B \mathbb{A}$ for some $\mathbb{A} \in \operatorname{Alg}_{\mathrm{As}}\left(\operatorname{Sp}^{\Sigma}(\mathbb{R})^{c}\right)$, where $B \mathbb{A}$ has one object $*$ with the morphism ring spectrum $B \mathbb{A}(*, *)=\mathbb{A})$. Then we have the filtered family (poset) of $\mathbb{R}$-spectral full subcategories $\left\{\left(\widehat{\mathcal{A}_{\lambda} \wedge_{\mathbb{R}}} \mathcal{B}\right)_{\mathrm{pe}}\right\}_{\lambda \in \Lambda}$ of $\left(\widehat{\mathcal{A} \wedge_{\mathbb{R}} \mathcal{B}}\right)_{\mathrm{pe}}$. The filtered colimit of this family in Cat $\mathrm{T}_{\mathbb{R}}^{\mathrm{pc}}\left[M^{-1}\right]$ is $\left(\widehat{\mathcal{A} \wedge_{\mathbb{R}} \mathcal{B}}\right)_{\text {pe }}$. Indeed, from the categorical equivalence $\operatorname{Cat}_{\mathbb{R}}^{\mathrm{pc}}\left[M^{-1}\right] \simeq \operatorname{Mod}_{\operatorname{Perf}_{R}}(\mathcal{S} \mathrm{t})$ and the conservative colimit-preserving functor $\operatorname{Mod}_{\operatorname{Perf}_{R}}(\mathcal{S} \mathrm{t}) \rightarrow \mathcal{S}$ t, if we transfer $\left\{\left(\widehat{\mathcal{A}_{\lambda} \wedge_{\mathbb{R}}} \mathcal{B}\right)_{\text {pe }}\right\}_{\lambda \in \Lambda}$ into the filtered family of stable idempotent-complete $\infty$-categories, we are reduced to proving that the colimit of the resulting diagram in $\mathcal{S}$ t is a stable $\infty$-category obtained from the spectral category $\left(\widehat{\mathcal{A} \wedge_{\mathbb{R}} \mathcal{B}}\right)_{\mathrm{pe}}$. We think of $\left\{\left(\widehat{\mathcal{A}_{\lambda} \wedge_{\mathbb{R}}} \mathcal{B}\right)_{\mathrm{pe}}\right\}_{\lambda \in \Lambda}$ as a filtered diagram (poset) of full subcategories of $\left(\widehat{\mathcal{A} \wedge_{\mathbb{R}} \mathcal{B}}\right)_{\mathrm{pe}}$. Since $\cup_{\lambda \in \Lambda}\left(\widehat{\mathcal{A}_{\lambda} \wedge_{\mathbb{R}}} \mathcal{B}\right)_{\mathrm{pe}}=\left(\widehat{\mathcal{A} \wedge_{\mathbb{R}} \mathcal{B}}\right)_{\mathrm{pe}}$, a colimit of this diagram in $\mathrm{Cat}_{\infty}$ is naturally equivalent to $\left(\widehat{\mathcal{A} \wedge_{\mathbb{R}}} \mathcal{B}\right)_{\text {pe }}$. Note that a filtered colimit of stable $\infty$-categories in $\mathcal{S}$ t naturally coincides with a colimit which is taken in $\mathrm{Cat}_{\infty}$ [25, Proposition 1.1.4.6]. It follows that $\left(\widehat{\mathcal{A} \wedge_{\mathbb{R}} \mathcal{B}}\right)_{\text {pe }}$ is a colimit of $\left\{\left(\widehat{\mathcal{A}_{\lambda} \wedge_{\mathbb{R}}} \mathcal{B}\right)_{\mathrm{pe}}\right\}_{\lambda \in \Lambda}$ in $\mathcal{S}$. . We then deduce that $\operatorname{colim}_{\lambda \in \Lambda}\left(\widehat{\mathcal{A}_{\lambda} \wedge_{\mathbb{R}}} \mathcal{B}\right)_{\text {pe }} \rightarrow\left(\widehat{\mathcal{A} \wedge_{\mathbb{R}} \mathcal{B}}\right)_{\text {pe }}$ is an equivalence in $\operatorname{Mod}_{\operatorname{Perf}_{R}}(\mathcal{S}$ t). In addition, we note that the tensor product in $\operatorname{Mod}_{\operatorname{Perf}_{R}}(\mathcal{S} \mathrm{t})$ preserves small colimits in each variable since $\mathcal{S} \mathrm{t}^{\otimes}$ is a symmetric monoidal compactly generated $\infty$-category whose tensor product preserves small colimits 
in each variable (see [6, Corollary 4.25]). Therefore, taking into account Proposition 6.3(3), we may and will suppose that $\mathcal{A}=B \mathbb{A}$ for some $\mathbb{A} \in \operatorname{Alg}_{\mathrm{As}}\left(\operatorname{Sp}^{\Sigma}(\mathbb{R})^{c}\right)$. Taking the same procedure to $\mathcal{B}$, we may and will suppose that $\mathcal{B}=B \mathbb{B}$ for some $\mathbb{B} \in \operatorname{Alg}_{\mathrm{As}}\left(\operatorname{Sp}^{\Sigma}(\mathbb{R})^{c}\right)$. We write $A$ and $B$ for the images of $\mathbb{A}$ and $\mathbb{B}$ in $\operatorname{Alg}_{\mathrm{As}}\left(\operatorname{Mod}_{R}\right)$, respectively. In this situation, we have a canonical equivalence $\pi(\mathcal{A}) \otimes_{R} \pi(\mathcal{B}) \simeq\left(\operatorname{RMod}_{A} \otimes_{R} \operatorname{RMod}_{B}\right)^{\omega} \simeq\left(\operatorname{RMod}_{A \otimes_{R} B}\right)^{\omega} \simeq\left(\widehat{B \mathbb{A}_{\mathbb{R}} \mathbb{B}}\right)_{\mathrm{pe}} \simeq$ $\pi\left(\mathcal{A} \wedge_{\mathbb{R}} \mathcal{B}\right)$ (cf. [5, Proposition 4.1(2)], [25, Theorem 4.8.5.16]), where $(-)^{\omega}$ indicates the full subcategory of compact objects. Hence $\pi: \mathrm{Cat}_{\mathbb{R}}^{\mathrm{pc}} \rightarrow \operatorname{Mod}_{B \mathbb{R}}\left(\operatorname{Cat}_{\mathbb{S}}^{\mathrm{pc}}\left[M^{-1}\right]\right)$ is symmetric monoidal, which induces a symmetric monoidal functor $\operatorname{Cat}_{\mathbb{R}}^{\mathrm{pc}}\left[M^{-1}\right]^{\otimes} \rightarrow \operatorname{Mod}_{B \mathbb{R}}^{\otimes}\left(\operatorname{Cat}_{\mathbb{S}}^{\mathrm{pc}}\left[M^{-1}\right]\right)$ whose underlying functor is a categorical equivalence. Thus we have the desired symmetric monoidal equivalence $\mathrm{Cat}_{\mathbb{R}}^{\mathrm{pc}}\left[M^{-1}\right]^{\otimes} \stackrel{\sim}{\rightarrow} \operatorname{Mod}_{B \mathbb{R}}^{\otimes}\left(\operatorname{Cat}_{\mathbb{S}}^{\mathrm{pc}}\left[M^{-1}\right]\right)$.

Using the equivalence in Proposition 6.7, we obtain equivalences of symmetric monoidal $\infty$-categories

$$
\theta: \quad \mathcal{S t}_{R}^{\otimes}=\operatorname{Mod}_{\operatorname{Perf}_{R}}^{\otimes}(\mathcal{S} \mathrm{t}) \simeq \operatorname{Mod}_{\operatorname{Perf}_{R}}^{\otimes}\left(\operatorname{Cat}_{\mathbb{S}}^{\mathrm{pc}}\left[M^{-1}\right]\right) \simeq \operatorname{Cat}_{\mathbb{R}}^{\mathrm{pc}}\left[M^{-1}\right]^{\otimes} .
$$

Next, to an $\mathbb{R}$-spectral category we assign Hochschild homology $R$-module spectrum endowed with circle action. The construction is based on the Hochschild-Mitchell cyclic nerves (cf. $[7,8]$ ).

Definition 6.8. Let $\mathcal{A}$ be a pointwise-cofibrant small $\mathbb{R}$-spectral category. Let $p \geq 0$ be a nonnegative integer. Let

$$
\mathcal{H} \mathcal{H}(\mathcal{A})_{p}:=\bigvee_{\left(X_{0}, \ldots, X_{p}\right)} \mathcal{A}\left(X_{p-1}, X_{p}\right) \wedge_{\mathbb{R}} \cdots \wedge_{\mathbb{R}} \mathcal{A}\left(X_{0}, X_{1}\right) \wedge_{\mathbb{R}} \mathcal{A}\left(X_{p}, X_{0}\right) .
$$

The coproduct is taken over the set of sequences $\left(X_{0}, \ldots, X_{p}\right)$ of objects of $\mathcal{A}$. The composition in $\mathcal{A}$ determines degeneracy maps $d_{0}, \ldots, d_{p}: \mathcal{H} \mathcal{H}(\mathcal{A})_{p} \rightarrow \mathcal{H} \mathcal{H}(\mathcal{A})_{p-1}$, and the unit map $\mathbb{S} \rightarrow$ $\mathcal{A}\left(X_{i}, X_{i}\right)$ determines face maps $s_{0}, \ldots, s_{p}: \mathcal{H} \mathcal{H}(\mathcal{A})_{p} \rightarrow \mathcal{H} \mathcal{H}(\mathcal{A})_{p+1}$. The cyclic permutation $\left(X_{0}, \ldots, X_{p}\right) \mapsto\left(X_{p}, X_{0}, \ldots, X_{p-1}\right)$ gives rise to the action of the cyclic group $\mathbf{Z} /(p+1) \mathbf{Z}$ on $\mathcal{H} \mathcal{H}(\mathcal{A})_{p}$. The family $\mathcal{H} \mathcal{H}(\mathcal{A})_{\bullet}:=\left\{\mathcal{H} \mathcal{H}(\mathcal{A})_{p}\right\}_{p \geq 0}$ equipped with degeneracy maps and face maps form a simplicial object in $\operatorname{Sp}^{\Sigma}(\mathbb{R})$. If we take into account the action of $\mathbf{Z} /(p+1) \mathbf{Z}$ on $\mathcal{H} \mathcal{H}(\mathcal{A})_{p}$, then $\mathcal{H} \mathcal{H}(\mathcal{A})_{\bullet}=\left\{\mathcal{H} \mathcal{H}(\mathcal{A})_{p}\right\}_{p \geq 0}$ is promoted to a cyclic object, that is, a functor $\Lambda^{\mathrm{op}} \rightarrow \mathrm{Sp}^{\Sigma}(\mathbb{R})$ from (the opposite category of) the cyclic category of Connes such that the composite $\Delta^{\mathrm{op}} \rightarrow \Lambda^{\mathrm{op}} \rightarrow \mathrm{Sp}^{\Sigma}(\mathbb{R})$ is the simplicial object. Here $\Lambda$ denotes the Connes' cyclic category, see [10, Section 2], [24] for the definition of the cyclic category.

We let Fun $\left(\Lambda^{\mathrm{op}}, \mathrm{Sp}^{\Sigma}(\mathbb{R})\right)$ denote the ordinary functor category from $\Lambda^{\text {op }}$ to $\mathrm{Sp}^{\Sigma}(\mathbb{R})$. The category $\operatorname{Fun}\left(\Lambda^{\mathrm{op}}, \mathrm{Sp}^{\Sigma}(\mathbb{R})\right)$ inherits a symmetric monoidal structure given by the pointwise tensor product $F \otimes G([n])=F([n]) \wedge_{\mathbb{R}} G([n])$.

From the definition of the tensor product of $\mathbb{R}$-spectral categories and the construction of $\mathcal{H} \mathcal{H}(\mathcal{A})_{p}$, it is straightforward to check that the assignment $\mathcal{A} \mapsto \mathcal{H} \mathcal{H}(\mathcal{A})$ • determines a symmetric monoidal functor

$$
\mathcal{H} \mathcal{H}(-) \bullet: \operatorname{Cat}_{\mathbb{R}}^{\mathrm{pc}} \rightarrow \operatorname{Fun}\left(\Lambda^{\mathrm{op}}, \operatorname{Sp}^{\Sigma}(\mathbb{R})\right) .
$$

The image of $\mathcal{H} \mathcal{H}(-)$ • is contained in $\operatorname{Fun}\left(\Lambda^{\mathrm{op}}, \mathrm{Sp}^{\Sigma}(\mathbb{R})^{c}\right)$ since the stable $\mathbb{R}$-model structure satisfies the axiom of symmetric monoidal model categories. Let $\operatorname{Sp}^{\Sigma}(\mathbb{R})^{c}\left[W^{-1}\right]$ be the symmetric monoidal $\infty$-category obtained from $\operatorname{Sp}^{\Sigma}(\mathbb{R})^{c}$ by inverting stable equivalences. The underlying $\infty$-category is presentable since $\operatorname{Sp}^{\Sigma}(\mathbb{R})$ is a combinatorial model category. There is a canonical symmetric monoidal functor Fun $\left(\Lambda^{\mathrm{op}}, \mathrm{Sp}^{\Sigma}(\mathbb{R})^{c}\right) \rightarrow \operatorname{Fun}\left(\Lambda^{\mathrm{op}}, \mathrm{Sp}^{\Sigma}(\mathbb{R})^{c}\left[W^{-1}\right]\right)$ induced by the symmetric monoidal functor $\mathrm{Sp}^{\Sigma}(\mathbb{R})^{c} \rightarrow \mathrm{Sp}^{\Sigma}(\mathbb{R})^{c}\left[W^{-1}\right]$.

We recall the following results from $[10,18,24]$ : 


\section{Lemma 6.9.}

(i) Let $\Lambda \rightarrow \bar{\Lambda}$ be the groupoid completion. Namely, it is induced by a unit map of the adjunction Cat $_{\infty} \rightleftarrows \mathcal{S}: \iota$, where $\iota$ is the fully faithful inclusion. Then $\bar{\Lambda}$ is equivalent to $B S^{1}$ in $\mathcal{S}$.

(ii) Let $\mathcal{C}$ be a presentable $\infty$-category. Let $F: \Lambda^{\mathrm{op}} \rightarrow \mathcal{C}$ be a cyclic object in $\mathcal{C}$. Let $F^{\prime}: B S^{1} \rightarrow \mathcal{C}$ be a functor. Let $\Delta^{0} \rightarrow B S^{1}$ be the map determined by the unique object of $B S^{1}$. Consider the commutative diagram

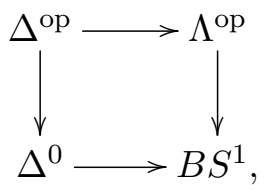

where we regard $\Delta^{0}$ as the groupoid completion of $\Delta^{\mathrm{op}}\left(\Delta^{\mathrm{op}}\right.$ is sifted so that the groupoid completion is given by the contractible space $\Delta^{0}$, cf. [27, Section 5.5.8]). Then $F^{\prime}$ is a left Kan extension of $F$ along $\Lambda^{\mathrm{op}} \rightarrow B S^{1}$ if and only if the composite $\Delta^{0} \rightarrow B S^{1} \rightarrow \mathcal{C}$ is a colimit of the composite $\Delta^{\mathrm{op}} \rightarrow \Lambda^{\mathrm{op}} \rightarrow \mathcal{C}$.

Proof. The first assertion is a result of Connes, see [10, Théorème 10], [18, 24]. The second assertion is proved in [18, Proposition 1.1].

Lemma 6.10. Let $R$ : Fun $\left(B S^{1}, \operatorname{Sp}^{\Sigma}(\mathbb{R})^{c}\left[W^{-1}\right]\right) \rightarrow \operatorname{Fun}\left(\Lambda^{\mathrm{op}}, \operatorname{Sp}^{\Sigma}(\mathbb{R})^{c}\left[W^{-1}\right]\right)$ be the functor induced by composition with $\Lambda^{\mathrm{op}} \rightarrow \bar{\Lambda}^{\mathrm{op}} \simeq B S^{1}$. Let

$$
L: \operatorname{Fun}\left(\Lambda^{\mathrm{op}}, \operatorname{Sp}^{\Sigma}(\mathbb{R})^{c}\left[W^{-1}\right]\right) \rightarrow \operatorname{Fun}\left(B S^{1}, \operatorname{Sp}^{\Sigma}(\mathbb{R})^{c}\left[W^{-1}\right]\right)
$$

be a left adjoint (the existence of a left adjoint follows from the adjoint functor theorem [27] and the fact that both $\infty$-categories are presentable). Then this left adjoint is symmetric monoidal.

Proof. Since the right adjoint is a symmetric monoidal functor, the left adjoint is an oplax symmetric monoidal functor. Thus it is enough to show that $L(F \otimes G) \rightarrow L(F) \otimes L(G)$ is an equivalence in $\operatorname{Fun}\left(B S^{1}, \operatorname{Sp}^{\Sigma}(\mathbb{R})^{c}\left[W^{-1}\right]\right)$ for $F, G: \Lambda^{\text {op }} \rightarrow \operatorname{Sp}^{\Sigma}(\mathbb{R})^{c}\left[W^{-1}\right]$. To this end, note first that $L(F), L(G): B S^{1} \rightarrow \mathrm{Sp}^{\Sigma}(\mathbb{R})^{c}\left[W^{-1}\right]$ are given by left Kan extensions of $F$ and $G$, respectively, along $\Lambda^{\mathrm{op}} \rightarrow B S^{1}$. By Lemma 6.9(ii), $F^{\prime}: B S^{1} \rightarrow \operatorname{Sp}^{\Sigma}(\mathbb{R})^{c}\left[W^{-1}\right]$ is a left Kan extension of $F: \Lambda^{\mathrm{op}} \rightarrow \mathrm{Sp}^{\Sigma}(\mathbb{R})^{c}\left[W^{-1}\right]$ if and only if the image of the unique object $*$ of $B S^{1}$ under $F^{\prime}$ is a colimit of the composite $F_{\Delta}: \Delta^{\mathrm{op}} \rightarrow \Lambda^{\mathrm{op}} \rightarrow \operatorname{Sp}^{\Sigma}(\mathbb{R})^{c}\left[W^{-1}\right]$. Let $G_{\Delta}$ be the restriciton of $G$ to $\Delta^{\mathrm{op}}$. Then $L(F \otimes G)(*)$ is a colimit of the composite $\Delta^{\mathrm{op}} \stackrel{\text { diag }}{\rightarrow} \Delta^{\mathrm{op}} \times \Delta^{\mathrm{op}} \stackrel{F_{\Delta} \times G_{\Delta}}{\rightarrow}$ $\mathrm{Sp}^{\Sigma}(\mathbb{R})^{c}\left[W^{-1}\right] \times \mathrm{Sp}^{\Sigma}(\mathbb{R})^{c}\left[W^{-1}\right] \stackrel{\otimes}{\rightarrow} \mathrm{Sp}^{\Sigma}(\mathbb{R})^{c}\left[W^{-1}\right]$. On the other hand, $L(F) \otimes L(G)(*)$ is a colimit of $\Delta^{\mathrm{op}} \times \Delta^{\mathrm{op}} \stackrel{F_{\Delta} \times G_{\Delta}}{\rightarrow} \operatorname{Sp}^{\Sigma}(\mathbb{R})^{c}\left[W^{-1}\right] \times \mathrm{Sp}^{\Sigma}(\mathbb{R})^{c}\left[W^{-1}\right] \stackrel{\otimes}{\rightarrow} \mathrm{Sp}^{\Sigma}(\mathbb{R})^{c}\left[W^{-1}\right]$ since the tensor product of $\operatorname{Sp}^{\Sigma}(\mathbb{R})^{c}\left[W^{-1}\right]$ preserves small colimits separately in each variable. Note that the diagonal functor $\Delta^{\mathrm{op}} \rightarrow \Delta^{\mathrm{op}} \times \Delta^{\mathrm{op}}$ is cofinal. Thus the canonical morphism $L(F \otimes G)(*) \rightarrow$ $L(F) \otimes L(G)(*)$ is an equivalence. This means that $L$ is symmetric monoidal.

Assembling these lemmata and constructions, we obtain a sequence of symmetric monoidal functors

$$
\begin{aligned}
H: \operatorname{Cat}_{\mathbb{R}}^{\mathrm{pc}} & \stackrel{\mathcal{H} \mathcal{H}(-)}{\longrightarrow} \operatorname{Fun}\left(\Lambda^{\mathrm{op}}, \operatorname{Sp}^{\Sigma}(\mathbb{R})^{c}\right) \rightarrow \operatorname{Fun}\left(\Lambda^{\mathrm{op}}, \operatorname{Sp}^{\Sigma}(\mathbb{R})^{c}\left[W^{-1}\right]\right) \\
& \simeq \operatorname{Fun}\left(\Lambda^{\mathrm{op}}, \operatorname{Mod}_{R}\right) \stackrel{L}{\rightarrow} \operatorname{Fun}\left(B S^{1}, \operatorname{Mod}_{R}\right) .
\end{aligned}
$$


More explicitly, the composition of $H$ and the forgetful functor $\operatorname{Fun}\left(B S^{1}, \operatorname{Mod}_{R}\right) \rightarrow \operatorname{Mod}_{R} \simeq$ $\mathrm{Sp}^{\Sigma}(\mathbb{R})^{c}\left[W^{-1}\right]$ sends a pointwise-cofibrant $\mathbb{R}$-spectral category $\mathcal{A}$ to the homotopy colimit of the simplicial object $\Delta^{\mathrm{op}} \hookrightarrow \Lambda^{\mathrm{op}} \stackrel{\mathcal{H}(\mathcal{H}(\mathcal{A})}{\longrightarrow} \operatorname{Sp}^{\Sigma}(\mathbb{R})$.

Next, we observe the invariance under Morita equivalences.

Lemma 6.11. Let $\mathcal{A}$ and $\mathcal{B}$ be pointwise-cofibrant $\mathbb{R}$-spectral categories. Let $F: \mathcal{A} \rightarrow \mathcal{B}$ be an $\mathbb{R}$-spectral functor which is a Morita equivalence. Then $H: \operatorname{Cat}_{\mathbb{R}}^{\mathrm{pc}} \rightarrow \operatorname{Fun}\left(B S^{1}, \operatorname{Mod}_{R}\right)$ carries $F$ to an equivalence in $\operatorname{Fun}\left(B S^{1}, \operatorname{Mod}_{R}\right)$.

Proof. It will suffice to prove that $H(F)$ is an equivalence in $\operatorname{Mod}_{R}$. By [8, Theorems 5.9 and 5.11], the image of $H(F)$ in $\operatorname{Mod}_{R} \simeq \operatorname{Sp}^{\Sigma}(\mathbb{R})\left[W^{-1}\right]$ is an equivalence if $F: \mathcal{A} \rightarrow \mathcal{B}$ is a Morita equivalence over $\mathbb{R}$. We explain the notion of a Morita equivalence over $\mathbb{R}$, which we distinguish from the notion of Morita equivalences for the moment. Let $\operatorname{Fun}_{\mathbb{R}}\left(\mathcal{A}^{\mathrm{op}}, \mathrm{Sp}^{\Sigma}(\mathbb{R})\right)$ be the $\mathbb{R}$-spectral category of $\mathbb{R}$-spectral functors. As in the case of $\mathbb{R}=\mathbb{S}$, it admits a combinatorial $\mathbb{R}$-spectral model structure whose weak equivalences (resp. fibrations) are objectwise stable equivalences (resp. fibrations). Let $\mathcal{D}(\mathcal{A})_{\mathbb{R}}$ denote the homotopy (triangulated) category of the full subcategory $\operatorname{Fun}_{\mathbb{R}}\left(\mathcal{A}^{\text {op }}, \operatorname{Sp}^{\Sigma}(\mathbb{R})\right)^{\text {cf }}$ spanned by cofibrant and fibrant objects. Let $\mathcal{D}_{\mathrm{pe}}(\mathcal{A})_{\mathbb{R}}$ be the smallest thick subcategory that contains the image of the Yoneda embedding $\mathcal{A} \rightarrow \mathcal{D}(\mathcal{A})_{\mathbb{R}}$. We define $\mathcal{D}_{\text {pe }}(\mathcal{B})_{\mathbb{R}}$ in a similar way. The functor $F$ induces $(\mathbb{L} F)_{\mathbb{R}}: \mathcal{D}_{\text {pe }}(\mathcal{A})_{\mathbb{R}} \rightarrow \mathcal{D}_{\text {pe }}(\mathcal{B})_{\mathbb{R}}$ as $F$ induces $\mathbb{L} F_{!}: \mathcal{D}_{\text {pe }}(\mathcal{A}) \rightarrow \mathcal{D}_{\text {pe }}(\mathcal{B})$. We say that $F$ is a Morita equivalence over $\mathbb{R}$ if $\left(\mathbb{L} F_{!}\right)_{\mathbb{R}}$ is an equivalence. Thus to prove our assertion, it is enough to show the following claim:

Claim 6.12. There exist equivalences $\mathcal{D}_{\mathrm{pe}}(\mathcal{A})_{\mathbb{R}} \simeq \mathcal{D}_{\mathrm{pe}}(\mathcal{A})$ and $\mathcal{D}_{\mathrm{pe}}(\mathcal{B})_{\mathbb{R}} \simeq \mathcal{D}_{\mathrm{pe}}(\mathcal{B})$ which identifies $\left(\mathbb{L} F_{!}\right)_{\mathbb{R}}$ with $\mathbb{L} F_{!}$up to natural equivalence. In particular, $F$ is a Morita equivalence over $\mathbb{R}$ if and only if $F$ is a Morita equivalence.

Proof. We let $\mathcal{D}_{\mathrm{pe}}^{\Sigma}(\mathcal{A})_{\mathbb{R}}$ be the full subcategory of $\operatorname{Fun}_{\mathbb{R}}\left(\mathcal{A}^{\mathrm{op}}, \mathrm{Sp}^{\Sigma}(\mathbb{R})\right)^{\mathrm{cf}}$ spanned by those objects that belongs to $\mathcal{D}_{\text {pe }}(\mathcal{A})_{\mathbb{R}}$. The Yoneda embedding $I: \mathcal{A} \rightarrow \mathcal{D}_{\text {pe }}^{\Sigma}(\mathcal{A})_{\mathbb{R}}$ (after replacing $\mathcal{A}$ by a fibrant one) induces $I_{!}: \mathcal{D}_{\text {pe }}^{\Sigma}(\mathcal{A}) \rightarrow \mathcal{D}_{\text {pe }}^{\Sigma}\left(\mathcal{D}_{\text {pe }}^{\Sigma}(\mathcal{A})_{\mathbb{R}}\right)$. By [6, Proposition 4.11], we deduce that the canonical functor $\mathcal{D}_{\mathrm{pe}}^{\Sigma}(\mathcal{A})_{\mathbb{R}} \rightarrow \mathcal{D}_{\mathrm{pe}}^{\Sigma}\left(\mathcal{D}_{\mathrm{pe}}^{\Sigma}(\mathcal{A})_{\mathbb{R}}\right)$ is a DK-equivalence. Thus we have $\mathcal{D}_{\mathrm{pe}}^{\Sigma}(\mathcal{A}) \rightarrow$ $\mathcal{D}_{\text {pe }}^{\Sigma}(\mathcal{A})_{\mathbb{R}}$ (in the homotopy category of Cat ). By the correspondence between stable idempotentcomplete $\infty$-categories and spectral categories up to Morita equivalences ([6, Theorems 4.22 and 4.23]), passing to stable (idempotent-complete) $\infty$-categories, we may replace $\mathcal{D}_{\text {pe }}^{\Sigma}(\mathcal{A}) \rightarrow$ $\mathcal{D}_{\text {pe }}^{\Sigma}(\mathcal{A})_{\mathbb{R}}$ by the induced exact functor of stable idempotent-complete $\infty$-categories which we denote by $f: \operatorname{Perf}(\mathcal{A}) \rightarrow \operatorname{Perf}(\mathcal{A})_{\mathbb{R}}$. Namely, it is enough to show that $f$ is an equivalence. Taking account of the Yoneda embedding, we see that $f$ is fully faithful on the full subcategory spanned by the image of $\mathcal{A}$. It follows that $f$ is fully faithful on the smallest stable subcategory of $\operatorname{Perf}(\mathcal{A})$, containing $\mathcal{A}$. Note that $\operatorname{Perf}(\mathcal{A})_{\mathbb{R}}$ is the idempotent completion of the smallest stable subcategory that contains the image of $\mathcal{A}$ under the Yoneda embedding. Then we conclude that $f$ is an equivalence. Similarly, $\mathcal{D}_{\text {pe }}(\mathcal{B})_{\mathbb{R}} \simeq \mathcal{D}_{\text {pe }}(\mathcal{B})$. Finally, using the construction of these equivalences and the functoriality of left adjoints $(-)$ !, we identify $\left(\mathbb{L} F_{\text {! }}\right)_{\mathbb{R}}$ with $\mathbb{L} F_{\text {! }}$.

Corollary 6.13. The symmetric monoidal functor $H: \operatorname{Cat}_{\mathbb{R}}^{\mathrm{pc}} \rightarrow \operatorname{Fun}\left(B S^{1}, \operatorname{Mod}_{R}\right)$ factors as

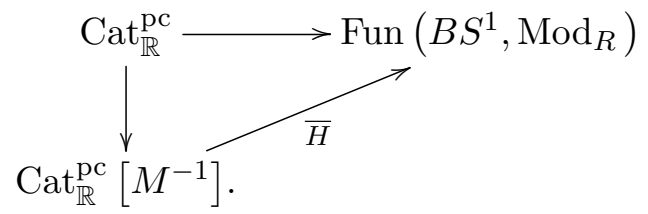

Proof. It follows from the universal property of $\mathrm{Cat}_{\mathbb{R}}^{\mathrm{pc}} \rightarrow \mathrm{Cat}_{\mathbb{R}}^{\mathrm{pc}}\left[M^{-1}\right]$ and Lemma 6.11. 
Composing with $\theta$, we obtain a sequence of symmetric monoidal functors

$$
\operatorname{Mod}_{\operatorname{Perf}_{R}}(\mathcal{S t}) \simeq \operatorname{Mod}_{\operatorname{Perf}_{R}}\left(\operatorname{Cat}_{\mathbb{S}}^{\mathrm{pc}}\left[M^{-1}\right]\right) \stackrel{\theta}{\longrightarrow} \operatorname{Cat}_{\mathbb{R}}^{\mathrm{pc}}\left[M^{-1}\right] \stackrel{\bar{H}}{\longrightarrow} \operatorname{Fun}\left(B S^{1}, \operatorname{Mod}_{R}\right) .
$$

Definition 6.14. Let $\mathcal{C}$ be a small $R$-linear stable $\infty$-category. We denote by $\mathcal{H} \mathcal{H} \bullet(\mathcal{C})$ the image of $\mathcal{C}$ in $\operatorname{Fun}\left(B S^{1}, \operatorname{Mod}_{R}\right)$ under the above composite $\bar{H} \circ \theta$. We often abuse notation by writing $\mathcal{H H}_{\bullet}(\mathcal{C})$ for its image in $\operatorname{Mod}_{R}$. We refer to $\mathcal{H}_{\bullet}(\mathcal{C})$ as Hochschild homology $R$-module spectrum of $\mathcal{C}$. If $\mathcal{A}$ is a pointwise-cofibrant $\mathbb{R}$-spectral category, we refer to the image $\bar{H}(\mathcal{A})$ in Fun $\left(B S^{1}, \operatorname{Mod}_{R}\right)$ or $\operatorname{Mod}_{R}$ as Hochschild homology $R$-module spectrum of $\mathcal{A}$.

We record our construction as a proposition:

Proposition 6.15. There is a sequence of symmetric monoidal functors

$$
\operatorname{Mod}_{\operatorname{Perf}_{R}}(\mathcal{S} \mathrm{t}) \stackrel{\theta}{\rightarrow} \operatorname{Cat}_{\mathbb{R}}^{\mathrm{pc}}\left[M^{-1}\right] \stackrel{\bar{H}}{\rightarrow} \operatorname{Fun}\left(B S^{1}, \operatorname{Mod}_{R}\right) \stackrel{\text { forget }}{\rightarrow} \operatorname{Mod}_{R},
$$

which to R-linear stable $\infty$-categories or pointwise-cofibrant $\mathbb{R}$-spectral categories assigns Hochschild homology $R$-module spectra. In particular, for any $\infty$-operad $\mathcal{O}$ it gives rise to

$$
\begin{aligned}
\operatorname{Alg}_{\mathcal{O}}\left(\operatorname{Mod}_{\operatorname{Perf}_{R}}(\mathcal{S t})\right) & \rightarrow \operatorname{Alg}_{\mathcal{O}}\left(\operatorname{Cat}_{\mathbb{R}}^{\mathrm{pc}}\left[M^{-1}\right]\right) \\
& \rightarrow \operatorname{Alg}_{\mathcal{O}}\left(\operatorname{Fun}\left(B S^{1}, \operatorname{Mod}_{R}\right)\right) \rightarrow \operatorname{Alg}_{\mathcal{O}}\left(\operatorname{Mod}_{R}\right) .
\end{aligned}
$$

\section{Construction}

In this section, we prove Theorem 7.14. Namely, we construct the structure of a KS-algebra on the pair of Hochschild cohomology spectrum and Hochschild homology spectrum. We maintain the notation of Section 6 .

Definition 7.1. Let $M$ and $M^{\prime}$ be (possibly empty) finite disjoint unions of open interval $(0,1)$ and the circle $\mathbf{R} / \mathbf{Z}=S^{1}$. That is, $M=(0,1)^{\sqcup m} \sqcup\left(S^{1}\right)^{\sqcup n}$ and $M^{\prime}=(0,1)^{\sqcup m^{\prime}} \sqcup\left(S^{1}\right)^{\sqcup n^{\prime}}$. Let $\mathrm{Emb}^{\text {rec }}\left(M, M^{\prime}\right)$ be the space of rectilinear embeddings. A rectilinear embedding $M \rightarrow M^{\prime}$ is an open embedding such that any restriction $(0,1) \rightarrow(0,1),(0,1) \rightarrow S^{1}$ and $S^{1} \rightarrow S^{1}$ is rectilinear, see Definition 4.1. The topology is induced from the compact-open topology (or parameters of rectilinear maps).

Let $\mathrm{Mfld}_{1}^{\text {rec }}$ be the the fibrant simplicial colored operad whose set $\left(\mathrm{Mfld}_{1}^{\text {rec }}\right)_{\text {col }}$ of colors consists of (possibly empty) finite disjoint unions of $(0,1)$ and $S^{1}$. For a finite family $\left\{M_{i}\right\}_{i \in I}$ of colors

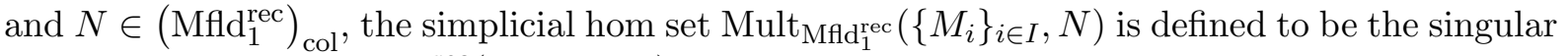
complex of the space $\operatorname{Emb}^{\text {rec }}\left(\sqcup_{i \in I} M_{i}, N\right)$ of rectilinear embeddings. The composition is defined in the obvious way. Then from Definition 4.7 , we obtain the associated $\infty$-operad $\left(\mathrm{Mfld}_{1}^{\text {rec }}\right)^{\otimes}$ $\rightarrow \Gamma$, which is a symmetric monoidal $\infty$-category by construction. Informally, objects of this symmetric monoidal $\infty$-category are finite disjoint unions of $(0,1)$ and $S^{1}$, and the symmetric monoidal structure is given by disjoint union. The empty space is a unit. The mapping spaces are spaces of rectilinear embeddings. Let $\mathrm{Mfld}_{1}^{\text {rec }}$ denote the underlying $\infty$-category. Let Disk ${ }_{1}^{\text {rec }} \subset$ $\mathrm{Mfld}^{\mathrm{rec}}$ be the full subcategory spanned by finite disjoint unions of $(0,1)$. It is closed under taking tensor products so that Disk ${ }_{1}^{\text {rec }}$ is promoted to a symmetric monoidal $\infty$-category $\left(\text { Disk }_{1}^{\text {red }}\right)^{\otimes}$ (it is equivalent to an ordinary symmetric monoidal 1-category).

Remark 7.2. There are several variants which are equivalent to $\mathrm{Mfld}_{1}^{\mathrm{rec}}$. Let $\mathrm{Mfld} \mathrm{fr}_{1}^{\mathrm{fr}}$ be the $\infty$-category of framed (or oriented) 1-manifolds without boundaries whose mapping spaces are spaces of embeddings of framed manifolds (see, e.g., [2, Section 2]). The symmetric monoidal

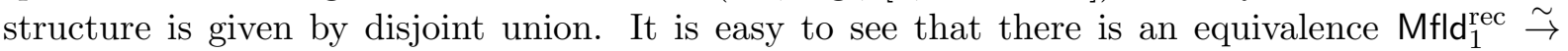
$\mathrm{Mfld}_{1}^{\mathrm{fr}}$ as symmetric monoidal $\infty$-categories. If we write Disk ${ }_{1}^{\mathrm{fr}}$ for the full subcategory of $\mathrm{Mfld}_{1}^{\mathrm{fr}}$ spanned by framed 1-disks, it also induces an equivalence Disk ${ }_{1}^{\text {rec }} \stackrel{\sim}{\rightarrow}$ Disk ${ }_{1}^{\text {fr }}$ of symmetric monoidal $\infty$-categories. 
From now on, for ease of notation, we write $M f l d_{1}$, Disk 1 , and Disk $_{1}^{\otimes}$ for $\mathrm{Mfld}_{1}^{\text {rec }}$, Disk $k_{1}^{\text {rec }}$, and $\left(\text { Disk }_{1}^{\text {rec }}\right)^{\otimes}$, respectively.

We set $\left(\right.$ Disk $\left._{1}\right) / S^{1}:=$ Disk $_{1} \times{ }_{\text {Mfld }_{1}}\left(\text { Mfld }_{1}\right)_{/ S^{1}}$. Let $\left\langle S^{1}\right\rangle$ be the full subcategory of Mfld $_{1}$ that consists of $S^{1}$. By the equivalence $\operatorname{Emb}^{\text {rec }}\left(S^{1}, S^{1}\right) \simeq S^{1}$, it follows that $\left\langle S^{1}\right\rangle$ is equivalent to $B S^{1}$, that is, the $\infty$-category which has one object $*$ together with the mapping space $\operatorname{Map}_{B S^{1}}(*, *)=S^{1}$ endowed with the composition law induced by the multiplication of $S^{1}$. Let Disk $1 /\left\langle S^{1}\right\rangle$ be the full subcategory of Fun $\left(\Delta^{1}, \mathrm{Mfld}_{1}\right)$ which consists of those functors $h: \Delta^{1} \rightarrow$ Mfld $_{1}$ such that $h(0) \in$ Disk $_{1}$ and $h(1) \in\left\langle S^{1}\right\rangle$. In other words, Disk $1 /\left\langle S^{1}\right\rangle=$ Disk $_{1} \times{ }_{\text {Mfld }_{1}}$ Fun $\left(\Delta^{1}\right.$, Mfld $\left._{1}\right) \times_{\text {Mfld }_{1}}\left\langle S^{1}\right\rangle$, where the functor from Fun $\left(\Delta^{1}, \mathrm{Mfld}_{1}\right)$ to the left $\mathrm{Mfld}_{1}$ (resp. the right $\mathrm{Mfld}_{1}$ ) is induced by the restriction to the source (resp. the target). The projection $\Delta^{0}=\left\{S^{1}\right\} \rightarrow\left\langle S^{1}\right\rangle=B S^{1}$ induces

$$
\begin{aligned}
\left(\text { Disk }_{1}\right)_{/ S^{1}} & \simeq \operatorname{Disk}_{1} \times_{\text {Mfld }_{1}} \operatorname{Fun}\left(\Delta^{1}, \operatorname{Mfld}_{1}\right) \times_{\text {Mfld }_{1}}\left\{S^{1}\right\} \\
& \rightarrow \operatorname{Disk}_{1} \times_{\text {Mfld }_{1}} \operatorname{Fun}\left(\Delta^{1}, \text { Mfld }_{1}\right) \times_{\text {Mfld }_{1}}\left\langle S^{1}\right\rangle=\operatorname{Disk}_{1} /\left\langle S^{1}\right\rangle,
\end{aligned}
$$

where the left categorical equivalence follows from [27, Proposition 4.2.1.5].

Lemma 7.3. Let Disk $_{1}^{\dagger}$ be the full subcategory of Disk ${ }_{1}$ spanned by nonempty spaces (namely, the empty space is omitted from Disk $\left.{ }_{1}\right)$. We set $\operatorname{Disk}_{1}^{\dagger} /\left\langle S^{1}\right\rangle=\operatorname{Disk}_{1}^{\dagger} \times_{\operatorname{Disk}_{1}}\left(\operatorname{Disk}_{1} /\left\langle S^{1}\right\rangle\right)$. Let $\Lambda$ be the cyclic category of Connes [10, Section 2]. There is an equivalence of categories $\Lambda^{\mathrm{op}} \simeq \operatorname{Disk}_{1}^{\dagger} /\left\langle S^{1}\right\rangle$.

Proof. This is a comparison between definitions which look different. We first recall that objects of $\Lambda$ are $(p)$ for $p \geq 0$, which is denoted by $\Lambda_{p}$ in [10]. Let $\left(S^{1}, p\right)$ be the circle $S^{1}=\mathbf{R} / \mathbf{Z}$ equipped with the set of torsion points $\frac{1}{p+1} \mathbf{Z} / \mathbf{Z}$. The hom set $\operatorname{Hom}_{\Lambda}((p),(q))$ is defined to be the set of homotopy classes of monotone degree one maps $\phi: S^{1} \rightarrow S^{1}$ such that $\phi\left(\frac{1}{p+1} \mathbf{Z} / \mathbf{Z}\right) \subset \frac{1}{q+1} \mathbf{Z} / \mathbf{Z}$. We denote points $\frac{0}{p+1}, \ldots, \frac{p}{p+1} \in \mathbf{R} / \mathbf{Z}$ by $x_{p}^{0}, \ldots, x_{p}^{p}$, respectively. Let $I_{p}^{i}=\left\{x_{p}^{i}<x<x_{p}^{i+1} \mid x \in[0,1)\right\}$ be the open set in $S^{1}$, where we use the obvious bijection of sets $[0,1) \simeq \mathbf{R} / \mathbf{Z}$. In what follows, we regard the superscripts in $x_{p}^{i}$ and $I_{p}^{i}$ as elements of $\mathbf{Z} /(p+1) \mathbf{Z}$. For $u, v \in[0,1),\{u<x<v\}$ means $\{x \in[0,1) \mid u<x<v\}$ if $u<v$, $\{x \in[0,1) \mid u<x<1,0 \leq x<v\}$ if $u>v>0$, and $\{x \in[0,1) \mid u<x<1\}$ if $u>v=0$. Given $(p) \in \Lambda$, we think of $j_{p}:(\mathbf{R} / \mathbf{Z}) \backslash\left(\frac{1}{p+1} \mathbf{Z} / \mathbf{Z}\right)=\left(I_{p}^{0} \sqcup \cdots \sqcup I_{p}^{p}\right) \hookrightarrow \mathbf{R} / \mathbf{Z}=S^{1}$ as an object of Disk ${ }_{1}^{\dagger} /\left\langle S^{1}\right\rangle$. We fix $I_{p}^{i} \simeq(0,1)$ such that $I_{p}^{i} \hookrightarrow \mathbf{R} / \mathbf{Z}$ is equivalent to $(0,1) \hookrightarrow \mathbf{R} \rightarrow \mathbf{R} / \mathbf{Z}$. We write $J(p)$ for it. We note that every object of $\operatorname{Disk}_{1}^{\dagger} /\left\langle S^{1}\right\rangle$ is equivalent to $J(p)$ for some $p \geq 0$. Since each component of $\operatorname{Map}_{\text {Mfld }_{1}}\left((0,1)^{\sqcup p+1}, S^{1}\right)$ is naturally equivalent to $S^{1}$, the computation of mapping spaces shows that $\operatorname{Disk}_{1}^{\dagger} /\left\langle S^{1}\right\rangle$ is equivalent to the nerve of a 1-category. We may and will abuse notation by identifying Disk ${ }_{1}^{\dagger} /\left\langle S^{1}\right\rangle$ with its homotopy category. Suppose that we are given a monotone degree one map $\phi: S^{1} \rightarrow S^{1}$ such that $\phi\left(\frac{1}{p+1} \mathbf{Z} / \mathbf{Z}\right) \subset \frac{1}{q+1} \mathbf{Z} / \mathbf{Z}$. Assume that $p \geq 1$ and $\phi\left(j_{p}\left(I_{p}^{i}\right)\right)$ is not a one-point space. Let $\sigma(i, \phi)$ be an element of $\mathbf{Z} /(p+1) \mathbf{Z}$ such that $x_{q}^{\sigma(i, \phi)}=\phi\left(x_{p}^{i}\right)$. Consider a rectilinear embedding

$$
\iota_{i, \phi}: I_{q}^{\sigma(i, \phi)} \sqcup I_{q}^{\sigma(i, \phi)+1} \sqcup \cdots \sqcup I_{q}^{\sigma(i+1, \phi)-1} \simeq(0,1) \sqcup \cdots \sqcup(0,1) \hookrightarrow(0,1) \simeq I_{p}^{i}
$$

such that $\iota_{i, \phi}\left(I_{q}^{\sigma(i, \phi)}\right)<\cdots<\iota_{i, \phi}\left(I_{q}^{\sigma(i+1, \phi)-1}\right)$ in $(0,1)$ (we here abuse notation: for two subsets $S, T \subset(0,1), S<T$ if $s<t$ for any pair $(s, t) \in S \times T)$. When $p=0$, we define $\iota_{i, \phi}$ by replacing $I_{q}^{\sigma(i, \phi)} \sqcup I_{q}^{\sigma(i, \phi)+1} \sqcup \cdots \sqcup I_{q}^{\sigma(i+1, \phi)-1}$ by $I_{q}^{\sigma(i, \phi)} \sqcup I_{q}^{\sigma(i, \phi)+1} \sqcup \cdots \sqcup I_{q}^{\sigma(i, \phi)-1}$. Such a rectilinear embedding is unique up to equivalences. Given $\phi \in \operatorname{Hom}_{\Lambda}((p),(q))$, we define the class of a map $\phi^{*}: J(q) \rightarrow J(p)$ in $\operatorname{Disk}_{1}^{\dagger} /\left\langle S^{1}\right\rangle$ such that the fiber of the induced morphism $I_{q}^{0} \sqcup \cdots \sqcup I_{q}^{q} \rightarrow I_{p}^{0} \sqcup \cdots \sqcup I_{p}^{p}$ over the connected component $I_{p}^{i}$ is (equivalent to) $\iota_{i, \phi}$ if $\phi\left(I_{p}^{i}\right)$ is not a one-point space, and if otherwise there is no component which maps to $I_{p}^{i}$. Notice that such 
a class is unique. It is routine to check that the assignments $(p) \mapsto J(p)$ and $\phi \mapsto \phi^{*}$ determine a categorical equivalence $\Lambda^{\mathrm{op}} \stackrel{\sim}{\rightarrow} \operatorname{Disk}_{1}^{\dagger} /\left\langle S^{1}\right\rangle$, where the target is identified with the homotopy category.

Lemma 7.4. Let $\pi$ : Disk ${ }_{1}^{\dagger} /\left\langle S^{1}\right\rangle \rightarrow\left\langle S^{1}\right\rangle$ be the projection. It is a groupoid completion of $\operatorname{Disk}_{1}^{\dagger} /\left\langle S^{1}\right\rangle$.

Proof. By Lemmas 6.9(i) and 7.3, there is a groupoid completion $c: \Lambda^{\mathrm{op}} \simeq \operatorname{Disk}_{1}^{\dagger} /\left\langle S^{1}\right\rangle \rightarrow$ $B S^{1}$. Thus, by the universal property, there is a canonical morphism from $c: \Lambda^{\text {op }} \rightarrow B S^{1}$

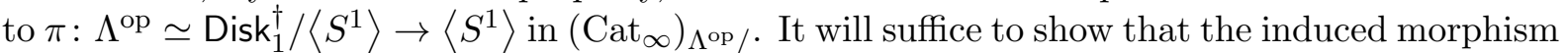
$g: B S^{1} \rightarrow\left\langle S^{1}\right\rangle \simeq B S^{1}$ is an equivalence, equivalently, it is induced by an equivalence $S^{1} \rightarrow S^{1}$ as $\mathbf{E}_{1}$-monoid spaces. To this end, assume that $g: B S^{1} \rightarrow\left\langle S^{1}\right\rangle \simeq B S^{1}$ is induced by a map $S^{1} \rightarrow S^{1}$ of degree $n$, where $|n|=p+1, p>0$. We will show that this gives rise to a contradiction. The automorphism group of $(p)$ in $\Lambda^{\mathrm{op}} \simeq \operatorname{Disk}_{1}^{\dagger} /\left\langle S^{1}\right\rangle$ is $\mathbf{Z} /(p+1) \mathbf{Z}$ so that there is the functor $h: B \mathbf{Z} /(p+1) \mathbf{Z} \rightarrow B S^{1}$ induced by $\pi$. By the factorization $\Lambda^{\text {op }} \stackrel{c}{\rightarrow} B S^{1} \stackrel{g}{\rightarrow}\left\langle S^{1}\right\rangle \simeq B S^{1}$ and our assumption, $h: B \mathbf{Z} /(p+1) \mathbf{Z} \rightarrow B S^{1}$ factors through the canonical morphism $\Delta^{0} \rightarrow B S^{1}$. Thus, the fiber product of $B \mathbf{Z} /(p+1) \mathbf{Z} \stackrel{h}{\rightarrow} B S^{1} \leftarrow \Delta^{0}=*$ is $B(\mathbf{Z} \times \mathbf{Z} /(p+1) \mathbf{Z})$. On the other hand, the space/ $\infty$-groupoid in $\left(\text { Disk }_{1}\right)_{/ S^{1}} \simeq$ Disk $_{1} /\left\langle S^{1}\right\rangle \times{ }_{\left\langle S^{1}\right\rangle} \Delta^{0}$ spanned by $J(p):(0,1)^{\sqcup p+1}$ $\rightarrow S^{1}$ (obtained by discarding non-invertible morphisms) is equivalent to $B \mathbf{Z}$. It gives rise to a contradiction $B(\mathbf{Z} \times \mathbf{Z} /(p+1) \mathbf{Z}) \simeq B \mathbf{Z}$.

Remark 7.5. There is another category relevant to the cyclic category: the paracyclic category $\Lambda_{\infty}$. Let us recall the definition of the paracyclic category. We follow [14]. The set of objects of $\Lambda_{\infty}$ is $\left\{(0)_{\infty},(1)_{\infty}, \ldots,(p)_{\infty}, \ldots\right\}_{p \geq 0}$. The hom set $\operatorname{Hom}_{\Lambda_{\infty}}\left((p)_{\infty},(q)_{\infty}\right)$ is defined to be the set of monotonically increasing maps $f: \mathbf{Z} \rightarrow \mathbf{Z}$ such that $f(i+k(p+1))=$ $f(i)+k(q+1)$ for any $k \in \mathbf{Z}$. We define a functor $\Lambda_{\infty} \rightarrow \Lambda$ which carries $(p)_{\infty}$ to $(p)$. The map $\operatorname{Hom}_{\Lambda_{\infty}}\left((p)_{\infty},(q)_{\infty}\right) \rightarrow \operatorname{Hom}_{\Lambda}((p),(q))$ carries $f$ to $\phi_{f}: S^{1} \rightarrow S^{1}$, where $\phi_{f}$ is the class of a map such that $\phi_{f}\left(x_{p}^{i}\right)=x_{q}^{f(i)} \in \frac{1}{q+1} \mathbf{Z} / \mathbf{Z}$ for $i \in \mathbf{Z} /(p+1) \mathbf{Z}$. Here, we regard $f(i)$ as belonging to $\mathbf{Z} /(q+1) \mathbf{Z}$. This determines a functor $\Lambda_{\infty} \rightarrow \Lambda$. Unwinding the definition of $\Lambda_{\infty} \rightarrow \Lambda$, we see that it is a (homotopy) quotient morphism $\Lambda_{\infty} \rightarrow \Lambda_{\infty} / B \mathbf{Z} \simeq \Lambda$ that comes from a free action of $B \mathbf{Z}$ on $\Lambda_{\infty}$. This free action of $B \mathbf{Z}$ is determined by the natural equivalence from the identity functor $\operatorname{id}_{\Lambda_{\infty}}$ to itself such that for any $p \geq 0$, the induced map $(p)_{\infty} \rightarrow(p)_{\infty}$ is the map $i \mapsto i+p+1$ (see [14] for details). The paracyclic category also has a geometric description. From the proof of Lemma 7.6 below, $\Delta^{\mathrm{op}} \rightarrow \Lambda^{\infty}$ is (left) cofinal so that it induces an equivalence between their groupoid completions. Since the groupoid completion of $\Delta^{\mathrm{op}}$ is contractible (note that it's sifted), the groupoid completion $\bar{\Lambda}_{\infty}$ of $\Lambda_{\infty}$ is a contractible space. It follows that the geometric realization of $\Lambda$ is equivalent to $B B \mathbf{Z}=B S^{1}$ (see also Lemma 6.9(i)). The composition with the opposite functor $\Lambda_{\infty}^{\mathrm{op}} \rightarrow \Lambda^{\mathrm{op}} \simeq \operatorname{Disk}_{1}^{\dagger} /\left\langle S^{1}\right\rangle \rightarrow\left\langle S^{1}\right\rangle=B S^{1}$ factors through the groupoid completion $\Lambda_{\infty}^{\mathrm{op}} \rightarrow \bar{\Lambda}_{\infty}^{\mathrm{op}} \simeq \Delta^{0}$. Consequently, we have the induced functor $\Lambda_{\infty}^{\mathrm{op}} \rightarrow\left(\text { Disk }_{1}^{\dagger}\right)_{/ S^{1}} \simeq \operatorname{Disk}_{1}^{\dagger} /\left\langle S^{1}\right\rangle \times{ }_{\left\langle S^{1}\right\rangle} \Delta^{0}$. This is an equivalence. Clearly, it is essentially surjective. The map $\operatorname{Hom}_{\Lambda_{\infty}^{\mathrm{op}}}\left((q)_{\infty},(p)_{\infty}\right) \rightarrow \operatorname{Hom}_{\Lambda^{\mathrm{op}}}((q),(p))$ is a homotopy quotient map that comes from a free action of $\mathbf{Z}$. We see that $\operatorname{Hom}_{\Lambda_{\infty}^{\mathrm{op}}}\left((q)_{\infty},(p)_{\infty}\right)$ is a (homotopy) fiber product $\operatorname{Hom}_{\Lambda_{\infty}^{\mathrm{op}}}\left((q)_{\infty},(p)_{\infty}\right) / \mathbf{Z} \times_{B \mathbf{Z}} \Delta^{0} \simeq \operatorname{Hom}_{\Lambda^{\mathrm{op}}}((q),(p)) \times_{B \mathbf{Z}} \Delta^{0}$. It follows that $\Lambda_{\infty}^{\mathrm{op}} \rightarrow\left(\text { Disk }_{1}^{\dagger}\right)_{/ S^{1}}$ is fully faithful. Hence $\Lambda_{\infty}^{\text {op }} \stackrel{\sim}{\rightarrow}\left(\text { Disk }_{1}^{\dagger}\right)_{/ S^{1}}$.

Lemma 7.6. Let $\mathcal{C}$ be a presentable $\infty$-category. Let $\Lambda_{\infty}$ be the paracyclic category, see [14] or Remark 7.5. Let $\Lambda_{\infty}^{\mathrm{op}} \simeq\left(\text { Disk }_{1}^{\dagger}\right)_{/ S^{1}} \rightarrow$ Disk $_{1}^{\dagger} /\left\langle S^{1}\right\rangle \simeq \Lambda^{\mathrm{op}}$ be the natural functor. Let $f:$ Disk $_{1}^{\dagger} /\left\langle S^{1}\right\rangle \rightarrow \mathcal{C}$ and $g:\left\langle S^{1}\right\rangle \rightarrow \mathcal{C}$ be functors and let $f \rightarrow \pi \circ g$ be a natural trans- 
formation. Then $g$ is a left Kan extension of $f$ along $\pi$ : Disk ${ }_{1}^{\dagger} /\left\langle S^{1}\right\rangle \rightarrow\left\langle S^{1}\right\rangle$ if and only if $\Delta^{0} \rightarrow B S^{1} \simeq\left\langle S^{1}\right\rangle \stackrel{g}{\rightarrow} \mathcal{C}$ determines a colimit of the composite $\left(\text { Disk }_{1}^{\dagger}\right)_{/ S^{1}} \rightarrow$ Disk $_{1}^{\dagger} /\left\langle S^{1}\right\rangle \stackrel{f}{\rightarrow} \mathcal{C}$.

Moreover, the inclusion $\left(\text { Disk }_{1}^{\dagger}\right)_{/ S^{1}} \rightarrow\left(\text { Disk }_{1}\right)_{/ S^{1}}$ is cofinal. Therefore, if we suppose that $f:$ Disk $_{1}^{\dagger} /\left\langle S^{1}\right\rangle \rightarrow \mathcal{C}$ is the restriction of a functor $\tilde{f}:$ Disk $_{1} /\left\langle S^{1}\right\rangle \rightarrow \mathcal{C}$, the above condition that $g$ is a left Kan extension of $f$ is also equivalent to the condition that $\Delta^{0} \rightarrow B S^{1} \rightarrow \mathcal{C}$ determines a colimit of the composite $\left(\text { Disk }_{1}\right)_{S^{1}} \rightarrow \operatorname{Disk}_{1} /\left\langle S^{1}\right\rangle \stackrel{\tilde{f}}{\rightarrow} \mathcal{C}$, where the first arrow $\left(\text { Disk }_{1}\right)_{/ S^{1}} \rightarrow$ Disk $_{1} /\left\langle S^{1}\right\rangle$ is the natural functor.

Proof. There is a faithful functor $\Delta^{\mathrm{op}} \rightarrow \Lambda_{\infty}^{\mathrm{op}}$ that is (left) cofinal [26, Proposition 4.2.8]: it is the same as the functor $m$ in Remark 7.7. Thus, for any paracyclic object $F: \Lambda_{\infty}^{\mathrm{op}} \rightarrow \mathcal{C}$, the canonical morphism $\operatorname{colim}_{[p] \in \Delta^{\text {op }}} F([p]) \rightarrow \operatorname{colim}_{(p)_{\infty} \in \Lambda_{\infty}^{\mathrm{op}}} F\left((p)_{\infty}\right)$ is an equivalence. Our first assertion now follows from this fact and Lemma 6.9(ii).

To prove the second assertion, it will suffice to prove that $\left(\left(\text { Disk }_{1}^{\dagger}\right)_{/ S^{1}}\right)_{e /}=\left(\text { Disk }_{1}^{\dagger}\right)_{/ S^{1}}$ $\times_{\left(\text {Disk }_{1}^{\dagger}\right)_{/ S^{1}}}\left(\left(\text { Disk }_{1}\right)_{/ S^{1}}\right)_{e /}$ is weakly contractible, where $e$ is the map $e: \phi \rightarrow S^{1}$ from the empty space to $S^{1}$. Since $e$ is an initial object in $\left(\text { Disk }_{1}\right)_{/ S^{1}}$, we are reduced to proving that $\left(\text { Disk }_{1}^{\dagger}\right)_{/ S^{1}}$ $\simeq \Lambda_{\infty}^{\mathrm{op}}$ is weakly contractible. By Quillen's theorem $\mathrm{A}$, it is clear because $\Delta^{\mathrm{op}}$ is weakly contractible and $\Delta^{\mathrm{op}} \rightarrow \Lambda_{\infty}^{\mathrm{op}}$ is (left) cofinal (it follows also from the fact that $\left(\text { Disk }_{1}^{\dagger}\right)_{/ S^{1}}$ is sifted).

Remark 7.7. We have the following commutative diagram of categories:

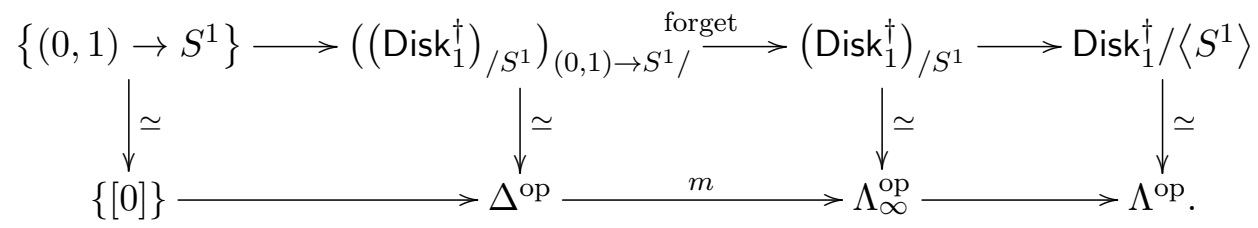

It is straightforward to observe that the composite $\left(\left(\text { Disk }_{1}^{\dagger}\right)_{/ S^{1}}\right)_{(0,1) \rightarrow S^{1} /} \rightarrow \Lambda^{\text {op }}$ is a faithful and essentially surjective functor whose image is $\Delta^{\mathrm{op}}$ contained in $\Lambda^{\mathrm{op}}$.

Let $\mathrm{Mfld}_{1}^{\mathrm{ic}}$ be the colored simplicial full suboperad of $\mathrm{Mfld}_{1}^{\text {rec }}$ (Definition 7.1) whose set $\left(\mathrm{Mfld}_{1}^{\mathrm{ic}}\right)_{\text {col }}$ of colors is $\left\{(0,1), S^{1}\right\}$. Namely, for $M_{i}, N \in\left\{(0,1), S^{1}\right\}$, the simplicial Hom set Mult $_{\mathrm{Mfld}_{1}^{\mathrm{ic}}}\left(\left\{M_{i}\right\}_{i \in I}, N\right)$ is the singular complex of the space $\operatorname{Emb}^{\mathrm{rec}}\left(\sqcup_{i \in I} M_{i}, N\right)$ of rectilinear embeddings. The superscript " $i c$ " stands for the "interval" and the "circle". Let Disk 1 be the full

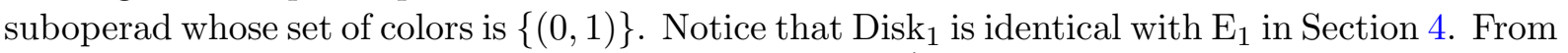

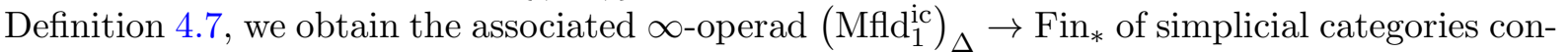
structed from Mfldic . Also, to $\mathrm{E}_{1}=\operatorname{Disk}_{1} \subset \mathrm{Mfld}_{1}^{\mathrm{ic}}$ we associate $\left(\mathrm{E}_{1}\right)_{\Delta}=\left(\operatorname{Disk}_{1}\right)_{\Delta} \rightarrow \mathrm{Fin}_{*}$.

Construction 7.8. We define $\rho:\left(\mathrm{E}_{1}\right)_{\Delta} \times\left(\mathrm{Mfld}_{1}^{\mathrm{ic}}\right)_{\Delta} \rightarrow \mathrm{DCyl}_{\Delta}$ which makes the following diagram commute

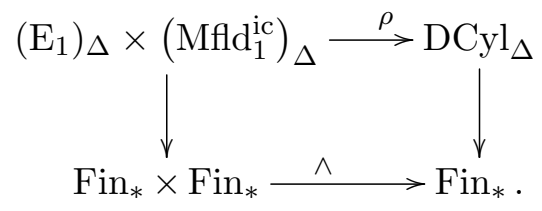

Here $\mathrm{DCyl}_{\Delta} \rightarrow \mathrm{Fin}_{*}$ is the map of simplicial categories associated to DCyl. The lower horizontal arrow $\wedge: \operatorname{Fin}_{*} \times \operatorname{Fin}_{*} \rightarrow \operatorname{Fin}_{*}$ that sends a pair $(\langle m\rangle,\langle n\rangle)$ to $\langle m n\rangle$ is defined in [25, Notation 2.2.5.1]. Let $X=\left(\langle m\rangle,\left(L_{1}, \ldots, L_{m}\right)\right)$ be an object of $\left(\mathrm{E}_{1}\right)_{\Delta}$, where $L_{s}=(0,1)$ for each $1 \leq s \leq m$. Let $Y=\left(\langle n\rangle,\left(M_{1}, \ldots, M_{n}\right)\right)$ be an object of $\left(\mathrm{Mfld}_{1}^{\text {ic }}\right)_{\Delta}$ where $M_{t}$ is either 
$(0,1)$ or $S^{1}$ for each $1 \leq t \leq n$. We define $\rho((X, Y))$ to be $\left(\langle m n\rangle,\left(L_{s} \times M_{t}\right)\right)_{\substack{1 \leq s \leq m \\ 1 \leq t \leq n}}$, where we abuse notation by the identifications $(0,1)^{2}=D$ and $(0,1) \times S^{1}=C$, see Definition 4.4.

$X^{\prime}=\left(\left\langle m^{\prime}\right\rangle,\left(L_{1}^{\prime}, \ldots, L_{m}^{\prime}\right)\right)$ be another object of $\left(\mathrm{E}_{1}\right)_{\Delta}$. Let $Y^{\prime}=\left(\left\langle n^{\prime}\right\rangle,\left(M_{1}^{\prime}, \ldots, M_{n}^{\prime}\right)\right)$ be another object of $\left(\mathrm{Mfld}_{1}^{\mathrm{ic}}\right)_{\Delta}$. Given a pair of morphisms $f:\langle m\rangle \rightarrow\left\langle m^{\prime}\right\rangle$ and $g:\langle n\rangle \rightarrow\left\langle n^{\prime}\right\rangle$, we define a map

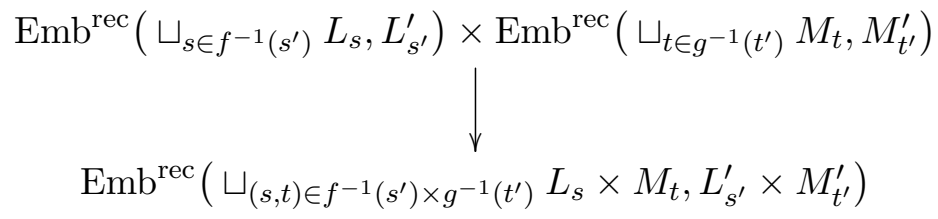

that sends $(\phi, \psi)$ to $\phi \times \psi$. Taking the product parameterized by $\left(s^{\prime}, t^{\prime}\right)$ with $1 \leq s^{\prime} \leq m^{\prime}$, $1 \leq t^{\prime} \leq n^{\prime}$ and taking simplicial nerves, we obtain morphisms of hom simplicial sets. It gives rise to a functor $\rho:\left(\mathrm{E}_{1}\right)_{\Delta} \times\left(\mathrm{Mfld}_{1}^{\mathrm{ic}}\right)_{\Delta} \rightarrow \mathrm{DCyl}_{\Delta}$ which makes the diagram commute. This construction is a natural extension of that in [25, Construction 5.1.2.1]: Let $\langle D\rangle \subset$ DCyl be the full suboperad whose set of colors is $\{D\}$. Let $\langle D\rangle_{\Delta} \rightarrow$ Fin $_{*}$ be the correpsonding simplicial full subcategory of $\mathrm{DCyl}_{\Delta}$. Then the restriction of $\rho$ induces $\left(\mathrm{E}_{1}\right)_{\Delta} \times\left(\mathrm{E}_{1}\right)_{\Delta} \rightarrow\langle D\rangle_{\Delta}$ lying over $\wedge: \operatorname{Fin}_{*} \times \operatorname{Fin}_{*} \rightarrow \mathrm{Fin}_{*}$, which is defined in loc. cit.

Let Mfld $_{1}$ be the simplicial nerve of $\left(\mathrm{Mfld}_{1}^{\mathrm{ic}}\right)_{\Delta}$. The simplicial nerves of the above diagrams give rise to the commutative diagram

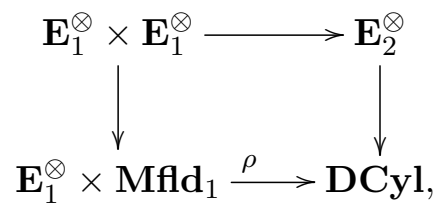

which lies over $\wedge: \Gamma \times \Gamma \rightarrow \Gamma$. We abuse notation by writing $\rho$ for the associated map.

Given an $\infty$-operad $\mathcal{O}^{\otimes} \rightarrow \Gamma$, there exist a symmetric monoidal $\infty$-category $\operatorname{Env}\left(\mathcal{O}^{\otimes}\right) \rightarrow \Gamma$ and a map $\mathcal{O}^{\otimes} \rightarrow \operatorname{Env}\left(\mathcal{O}^{\otimes}\right)$ of $\infty$-operads such that for any symmetric monoidal $\infty$-category $\mathcal{D}^{\otimes}$, the composition induces a categorical equivalence $\operatorname{Fun}^{\otimes}\left(\operatorname{Env}\left(\mathcal{O}^{\otimes}\right), \mathcal{D}^{\otimes}\right) \stackrel{\sim}{\rightarrow} \operatorname{Alg}_{\mathcal{O}^{\otimes}}\left(\mathcal{D}^{\otimes}\right)$, see [25, Section 2.2.4]. Here $\operatorname{Fun}^{\otimes}\left(\operatorname{Env}\left(\mathcal{O}^{\otimes}\right), \mathcal{D}^{\otimes}\right)$ denotes the $\infty$-category of symmetric monoidal functors. We shall refer to $\operatorname{Env}\left(\mathcal{O}^{\otimes}\right)$ as the symmetric monoidal envelope of $\mathcal{O}^{\otimes}$ (in loc. cit., it is referred to as the $\Gamma$-monoidal envelope). Through the categorical equivalence, for a map of $\infty$ operads $f: \mathcal{O}^{\otimes} \rightarrow \mathcal{D}^{\otimes}$, there exists a symmetric monoidal functor $\tilde{f}: \operatorname{Env}\left(\mathcal{O}^{\otimes}\right) \rightarrow \mathcal{D}^{\otimes}$ which is unique up to a contractible space of choices. We refer to $\tilde{f}$ as a symmetric monoidal functor that corresponds to $f$. Let Oper $_{\infty}$ be the $\infty$-category of (small) $\infty$-operads [25, Section 2.1.4] and let $\mathrm{Cat}_{\infty}^{\otimes}$ be the $\infty$-category of (small) symmetric monoidal $\infty$-categories whose morphisms are symmetric monoidal functors. Then the construction of symmetric monoidal envelopes gives a left adjoint $\mathrm{Oper}_{\infty} \rightarrow \mathrm{Cat}_{\infty}^{\otimes}$ of the canonical functor $\mathrm{Cat}_{\infty}^{\otimes} \rightarrow \mathrm{Oper}_{\infty}$. Here are some examples. The symmetric monoidal envelope $\widetilde{\mathbf{E}}_{1}^{\otimes}$ of $\mathbf{E}_{1}^{\otimes}$ is equivalent to Disk ${ }_{1}^{\otimes}$ as symmetric monoidal $\infty$-categories. Similarly, a symmetric monoidal envelope $\widetilde{\mathbf{E}}_{2}^{\otimes}$ of $\mathbf{E}_{2}^{\otimes}$ is equivalent to the symmetric monoidal $\infty$-category Disk $_{2}^{\otimes}$ of (possibly empty) finite disjoint unions of $(0,1)^{2}$ defined as in the case of Disk ${ }_{1}^{\otimes}$ : mapping spaces are spaces of rectilinear embeddings, and the tensor product is again given by disjoint union. Another quick example of symmetric monoidal envelopes is $\mathbf{M f l d}_{1} \rightarrow \mathrm{Mfld}_{1}^{\otimes}$.

Construction 7.9. Let $q: \mathcal{C}^{\otimes} \rightarrow \Gamma$ be a symmetric monoidal $\infty$-category. Let $p: \mathcal{P}^{\otimes} \rightarrow \Gamma$ be a symmetric monoidal $\infty$-category (resp. an $\infty$-operad). We construct a symmetric monoidal structure on the $\infty$-category $\operatorname{Fun}^{\otimes}\left(\mathcal{P}^{\otimes}, \mathcal{C}^{\otimes}\right)$ of symmetric monoidal functors (resp. the $\infty$ category $\operatorname{Alg}_{\mathcal{P} \otimes}\left(\mathcal{C}^{\otimes}\right)$ of algebra objects), see [25, Section 3.2.4] for more details of the case 
of $\operatorname{Alg}_{\mathcal{P} \otimes}\left(\mathcal{C}^{\otimes}\right)$. We define a map $\operatorname{Fun}^{\otimes}\left(\mathcal{P}^{\otimes}, \mathcal{C}^{\otimes}\right)^{\otimes} \rightarrow \Gamma\left(\right.$ resp. $\left.\operatorname{Alg}_{\mathcal{P}^{\otimes}}^{\otimes}\left(\mathcal{C}^{\otimes}\right) \rightarrow \Gamma\right)$ by the universal property that for any $\alpha: K \rightarrow \Gamma$, the set of morphisms $K \rightarrow \operatorname{Fun}^{\otimes}\left(\mathcal{P}^{\otimes}, \mathcal{C}^{\otimes}\right)^{\otimes}$ over $\Gamma$ (resp. $K \rightarrow \operatorname{Alg}_{\mathcal{P} \otimes}^{\otimes}\left(\mathcal{C}^{\otimes}\right)$ over $\Gamma$ ) is defined to be the set of morphisms $f: K \times \mathcal{P}^{\otimes} \rightarrow \mathcal{C}^{\otimes}$ such that

(i) the diagram

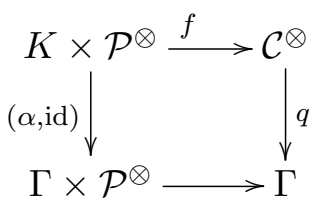

commutes; here the lower horizontal arrow is induced by $\wedge: \Gamma \times \Gamma \rightarrow \Gamma$,

(ii) for any vertex $k$ of $K$ and any $p$-coCartesian edge $\phi$ in $\mathcal{P}^{\otimes}, f(k, \phi)$ is a $q$-coCartesian edge (resp. for any vertex $k$ of $K$ and any inert morphism $\phi$ in $\mathcal{P}^{\otimes}, f(k, \phi)$ is an inert morphism in $\left.\mathcal{C}^{\otimes}\right)$.

The morphism $\operatorname{Alg}_{\mathcal{P} \otimes}^{\otimes}\left(\mathcal{C}^{\otimes}\right) \rightarrow \Gamma$ is a symmetric monoidal $\infty$-category whose underlying $\infty$-category is $\operatorname{Alg}_{\mathcal{P} \otimes}(\mathcal{C})$, cf. [25, Proposition 3.2.4.3]. Similarly, $\operatorname{Fun}^{\otimes}\left(\mathcal{P}^{\otimes}, \mathcal{C}^{\otimes}\right)^{\otimes} \rightarrow \Gamma$ is a symmetric monoidal $\infty$-category whose underlying $\infty$-category is $\operatorname{Fun}^{\otimes}\left(\mathcal{P}^{\otimes}, \mathcal{C}^{\otimes}\right)$ : the proof of $[25$, Proposition 3.2.4.3] based on the theory of categorical patterns can also be applied to $\operatorname{Fun}^{\otimes}\left(\mathcal{P}^{\otimes}, \mathcal{C}^{\otimes}\right)^{\otimes}$. An edge $\Delta^{1} \rightarrow \operatorname{Fun}^{\otimes}\left(\mathcal{P}^{\otimes}, \mathcal{C}^{\otimes}\right)^{\otimes}$ is a coCartesian edge if and only if for any $X \in \mathcal{P}$, the composite $\Delta^{1} \times\{X\} \subset \Delta^{1} \times \mathcal{P}^{\otimes} \rightarrow \mathcal{C}^{\otimes}$ determines a $q$-coCartesian edge (this means that the tensor product $F \otimes G$ of two symmetric monoidal functors $F: \mathcal{P}^{\otimes} \rightarrow \mathcal{C}^{\otimes}$ and $G: \mathcal{P}^{\otimes} \rightarrow \mathcal{C}^{\otimes}$ is informally given by objectwise tensor products $(F \otimes G)(X)=F(X) \otimes G(X))$.

Let $\mathcal{O}^{\otimes} \rightarrow \Gamma$ be an $\infty$-operad and let $\widetilde{\mathcal{O}}^{\otimes} \rightarrow \Gamma$ be the symmetric monoidal envelope. The composition with the inclusion $\mathcal{O}^{\otimes} \rightarrow \widetilde{\mathcal{O}}^{\otimes}$ induces a map over $\Gamma$

$$
\operatorname{Fun}^{\otimes}\left(\widetilde{\mathcal{O}}^{\otimes}, \mathcal{C}^{\otimes}\right)^{\otimes} \rightarrow \operatorname{Alg}_{\mathcal{O}}^{\otimes}\left(\mathcal{C}^{\otimes}\right)
$$

that preserves coCartesian edges, namely, it is a symmetric monoidal functor. Since the underlying functor is an equivalence [25, Proposition 2.2.4.9], it gives rise to a symmetric monoidal equivalence. That is, the categorical equivalence $\operatorname{Fun}^{\otimes}\left(\widetilde{\mathcal{O}}^{\otimes}, \mathcal{C}^{\otimes}\right) \simeq \operatorname{Alg}_{\mathcal{O} \otimes}\left(\mathcal{C}^{\otimes}\right)$ is promoted to a symmetric monoidal equivalence in the natural way.

Let $A$ be an $\mathbf{E}_{2}$-algebra in $\operatorname{Mod}_{R}$. By definition, it is a map of $\infty$-operads $A: \mathbf{E}_{2}^{\otimes} \rightarrow \operatorname{Mod}_{R}^{\otimes}$ over $\Gamma$. We denote by

$$
i_{!}(A): \mathbf{D C y l} \rightarrow \operatorname{Mod}_{R}^{\otimes}
$$

the operadic left Kan extension of $A$ along the inclusion $i: \mathbf{E}_{2}^{\otimes} \hookrightarrow \mathbf{D C y l}$. If we think of the color $S^{1}$ as an object in the fiber $\left.(\mathbf{M f l d})_{1}\right)_{\langle 1\rangle}$ of $\mathbf{M f l d} \mathbf{d}_{1} \rightarrow \Gamma$ over $\langle 1\rangle$, the full subcategory $\left\langle S^{1}\right\rangle$ spanned by $S^{1}$ determines the inclusion $\iota: B S^{1} \simeq\left\langle S^{1}\right\rangle \hookrightarrow\left(\mathbf{M f l d}_{1}\right)_{\langle 1\rangle} \subset \mathbf{M f l d}_{1}$. Then we have the following diagram

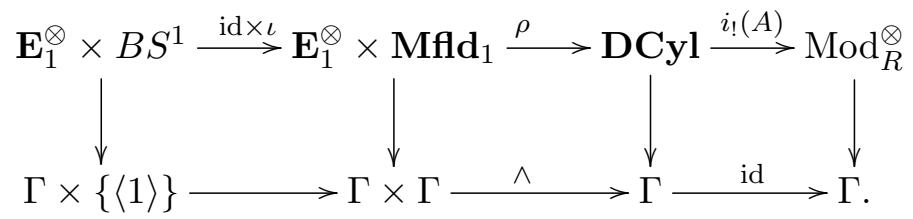

See Construction 7.8 for $\rho$. The composite $\Gamma \simeq \Gamma \times\{\langle 1\rangle\} \rightarrow \Gamma$ of lower horizontal arrows is the identity map. Note that the composite $\rho \circ(\mathrm{id} \times \iota): \mathbf{E}_{1}^{\otimes} \times B S^{1} \rightarrow \mathbf{D C y l}$ is the map 
$z: \mathbf{E}_{1}^{\otimes} \times B S^{1} \rightarrow \mathbf{C y l} \subset \mathbf{D C y l}$ which was defined in the discussion before Proposition 4.16. Taking into account the above diagram, Proposition 4.16 and Lemma 4.14, we have the induced functors

$$
\operatorname{Alg}_{\mathbf{D C y l}}\left(\operatorname{Mod}_{R}\right) \rightarrow \operatorname{Alg}_{\mathbf{C y l}}\left(\operatorname{Mod}_{R}\right) \stackrel{\sim}{\rightarrow} \operatorname{Alg}_{\mathbf{E}_{1}}\left(\operatorname{Fun}\left(B S^{1}, \operatorname{Mod}_{R}\right)\right),
$$

and we write $i_{!}(A)_{C}$ for the image of $i_{!}(A)$ in $\operatorname{Alg}_{\mathbf{E}_{1}}\left(\operatorname{Fun}\left(B S^{1}, \operatorname{Mod}_{R}\right)\right) \simeq \operatorname{Alg}_{\mathbf{C y l}}\left(\operatorname{Mod}_{R}\right)$.

Remark 7.10. The image of $C=(0,1) \times S^{1}$ under $i_{!}(A): \mathbf{D C y l} \rightarrow \operatorname{Mod}_{R}^{\otimes}$ can be viewed as the factorization homology $\int_{C} A$ in $\operatorname{Mod}_{R}$ in this context, cf. [2, 25].

We continue to suppose that $A$ is an $\mathbf{E}_{2}$-algebra in $\operatorname{Mod}{ }_{R}$. Let us consider the Hochschild homology $R$-module spectrum of $A$ defined as follows. Let $\operatorname{Alg}_{\text {As }}\left(\operatorname{Sp}^{\Sigma}(\mathbb{R})^{c}\right)$ be the category of associative algebra objects of $\operatorname{Sp}^{\Sigma}(\mathbb{R})^{c}$, where $\mathbb{R}$ be a (cofibrant) commutative symmetric ring spectrum that represents $R$, and $\operatorname{Sp}^{\Sigma}(\mathbb{R})^{c}$ is the full subcategory of $\operatorname{Sp}^{\Sigma}(\mathbb{R})$ spanned by cofibrant objects (cf. Section 6). The ordinary category $\operatorname{Alg}_{\mathrm{As}}\left(\operatorname{Sp}^{\Sigma}(\mathbb{R})^{c}\right)$ admits a symmetric monoidal structure given by $\mathbb{A} \otimes \mathbb{B}=\mathbb{A} \wedge_{\mathbb{R}} \mathbb{B}$. Define a symmetric monoidal functor $\operatorname{Alg}_{\mathrm{As}}\left(\operatorname{Sp}^{\Sigma}(\mathbb{R})^{c}\right) \rightarrow$ $\mathrm{Cat}_{\mathbb{R}}^{\mathrm{pc}}$ which carries $\mathbb{A}$ to $B \mathbb{A}$, where $B \mathbb{A}$ is the $\mathbb{R}$-spectral category having one object $*$ with the morphism spectrum $\mathbb{A}=B \mathbb{A}(*, *)$. We define $\mathcal{H} \mathcal{H}_{\bullet}(\mathbb{A})$ to be the Hochschild homology $R$-module spectrum of $B \mathbb{A}$. Namely, we use canonical symmetric monoidal functors

$$
\operatorname{Alg}_{\mathrm{As}}\left(\mathrm{Sp}^{\Sigma}(\mathbb{R})^{c}\right) \rightarrow \mathrm{Cat}_{\mathbb{R}}^{\mathrm{pc}} \rightarrow \mathrm{Cat}_{\mathbb{R}}^{\mathrm{pc}}\left[M^{-1}\right] \stackrel{\bar{H}}{\longrightarrow} \operatorname{Fun}\left(B S^{1}, \operatorname{Mod}_{R}\right),
$$

see Corollary 6.13. By inverting weak equivalences we obtain symmetric monoidal functors

$$
\operatorname{Alg}_{\mathrm{As}}\left(\operatorname{Mod}_{R}\right) \simeq \operatorname{Alg}_{\mathrm{As}}\left(\operatorname{Sp}^{\Sigma}(\mathbb{R})^{c}\right)\left[W^{-1}\right] \rightarrow \mathrm{Cat}_{\mathbb{R}}^{\mathrm{pc}}\left[M^{-1}\right] \rightarrow \operatorname{Fun}\left(B S^{1}, \operatorname{Mod}_{R}\right),
$$

see Example 6.4 for the first symmetric equivalence. This functor sends $A \in \operatorname{Alg}_{\text {As }}\left(\operatorname{Mod}_{R}\right)$ to $\mathcal{H} \mathcal{H}_{\bullet}(A)$. Note that there is a canonical categorical equivalence

$$
\operatorname{Alg}_{\mathbf{E}_{2}}\left(\operatorname{Mod}_{R}\right) \simeq \operatorname{Alg}_{\mathrm{As}} \operatorname{Alg}_{\mathrm{As}}\left(\operatorname{Mod}_{R}\right)
$$

that follows from the trivial fibration $\mathbf{E}_{1}^{\otimes} \rightarrow \mathrm{As}^{\otimes}$ and the equivalence $\mathbf{E}_{2}^{\otimes} \simeq \mathbf{E}_{1}^{\otimes} \otimes \mathbf{E}_{1}^{\otimes}$ (Dunn additivity theorem). Thus, we have the induced functor

$$
\operatorname{Alg}_{\mathbf{E}_{2}}\left(\operatorname{Mod}_{R}\right) \simeq \operatorname{Alg}_{\mathrm{As}} \operatorname{Alg}_{\mathrm{As}}\left(\operatorname{Mod}_{R}\right) \rightarrow \operatorname{Alg}_{\mathrm{As}}\left(\operatorname{Fun}\left(B S^{1}, \operatorname{Mod}_{R}\right)\right) .
$$

Given $A \in \operatorname{Alg}_{\mathbf{E}_{2}}\left(\operatorname{Mod}_{R}\right)$, we define $\mathcal{H} \mathcal{H} \bullet(A)$ to be the image of $A$ in $\operatorname{Alg}_{\text {As }}\left(\operatorname{Fun}\left(B S^{1}, \operatorname{Mod}_{R}\right)\right)$.

Proposition 7.11. There is a canonical equivalence

$$
\mathcal{H}_{\bullet}(A) \stackrel{\sim}{\rightarrow} i_{!}(A)_{C}
$$

in $\operatorname{Alg}_{\mathrm{As}}\left(\operatorname{Fun}\left(B S^{1}, \operatorname{Mod}_{R}\right)\right)$.

Let us consider $\operatorname{Alg}_{\mathrm{As}}\left(\operatorname{Sp}^{\Sigma}(\mathbb{R})^{c}\right) \stackrel{B(-)}{\longrightarrow} \mathrm{Cat}_{\mathbb{R}}^{\mathrm{pc}} \stackrel{\mathcal{H \mathcal { H } ( - )}}{\longrightarrow} \operatorname{Fun}\left(\Lambda^{\mathrm{op}}, \operatorname{Sp}^{\Sigma}(\mathbb{R})^{c}\right)$, see Definition 6.8 for $\mathcal{H} \mathcal{H}(-)$ •. We write $\mathcal{H H}_{\bullet}^{\Lambda}(-)$ for the composite. Let $\widetilde{\mathrm{As}}^{\otimes}$ be the symmetric monoidal envelope of $\mathrm{As}^{\otimes}$. The equivalence $\mathbf{E}_{1}^{\otimes} \stackrel{\sim}{\rightarrow}$ As induces Disk ${ }_{1}^{\otimes} \stackrel{\sim}{\rightarrow} \widetilde{\mathrm{As}}^{\otimes}$. There is a canonical symmetric monoidal equivalence $\operatorname{Fun}^{\otimes}\left(\widetilde{\mathrm{As}}^{\otimes},\left(\mathrm{Sp}^{\Sigma}(\mathbb{R})^{c}\right)^{\otimes}\right) \simeq \operatorname{Alg}_{\mathrm{As}}\left(\mathrm{Sp}^{\Sigma}(\mathbb{R})^{c}\right)$, see Construction 7.9. We write Disk $4 \hookrightarrow$ Disk $_{1}^{\otimes}$ for the inclusion of the fiber of the coCartesin fibration Disk ${ }_{1}^{\otimes} \rightarrow \Gamma$ over $\langle 1\rangle$. Using Lemma 7.3 , we have

$$
\xi: \Lambda^{\mathrm{op}} \simeq \operatorname{Disk}_{1}^{\dagger} /\left\langle S^{1}\right\rangle \stackrel{\text { forget }}{\longrightarrow} \operatorname{Disk}_{1} \hookrightarrow \text { Disk }_{1}^{\otimes} \simeq \widetilde{\mathrm{As}}^{\otimes} .
$$

The composition with $\xi$ induces

$$
g: \operatorname{Alg}_{\mathrm{As}}\left(\mathrm{Sp}^{\Sigma}(\mathbb{R})^{c}\right) \simeq \operatorname{Fun}^{\otimes}\left(\widetilde{\mathrm{As}}^{\otimes},\left(\mathrm{Sp}^{\Sigma}(\mathbb{R})^{c}\right)^{\otimes}\right) \rightarrow \operatorname{Fun}\left(\Lambda^{\mathrm{op}}, \operatorname{Sp}^{\Sigma}(\mathbb{R})^{c}\right),
$$

which is a symmetric monoidal functor. 
Lemma 7.12. The functor $\mathcal{H H}_{\bullet}^{\Lambda}(-): \operatorname{Alg}_{\mathrm{As}}\left(\mathrm{Sp}^{\Sigma}(\mathbb{R})^{c}\right) \rightarrow \operatorname{Fun}\left(\Lambda^{\mathrm{op}}, \mathrm{Sp}^{\Sigma}(\mathbb{R})^{c}\right)$ can be identified with

$$
g: \operatorname{Alg}_{\mathrm{As}}\left(\operatorname{Sp}^{\Sigma}(\mathbb{R})^{c}\right) \simeq \operatorname{Fun}^{\otimes}\left(\widetilde{\mathrm{As}}^{\otimes},\left(\operatorname{Sp}^{\Sigma}(\mathbb{R})^{c}\right)^{\otimes}\right) \rightarrow \operatorname{Fun}\left(\Lambda^{\mathrm{op}}, \operatorname{Sp}^{\Sigma}(\mathbb{R})^{c}\right)
$$

in the natural way. In particular, $\operatorname{Alg}_{\mathrm{As}}\left(\mathrm{Sp}^{\Sigma}(\mathbb{R})^{c}\right)\left[W^{-1}\right] \rightarrow \mathrm{Fun}\left(\Lambda^{\mathrm{op}}, \mathrm{Sp}^{\Sigma}(\mathbb{R})^{c}\left[W^{-1}\right]\right)$ induced by $\mathcal{H} \mathcal{H}(-)$ • can be identified with

$$
\operatorname{Alg}_{\mathrm{As}}\left(\operatorname{Sp}^{\Sigma}(\mathbb{R})^{c}\right)\left[W^{-1}\right] \simeq \operatorname{Fun}^{\otimes}\left(\widetilde{\operatorname{As}}^{\otimes}, \operatorname{Sp}^{\Sigma}(\mathbb{R})^{c}\left[W^{-1}\right]^{\otimes}\right) \rightarrow \operatorname{Fun}\left(\Lambda^{\mathrm{op}}, \operatorname{Sp}^{\Sigma}(\mathbb{R})^{c}\left[W^{-1}\right]\right)
$$

induced by the composition with $\xi$.

Proof. We use the notation in Lemma 7.3. Let $\phi_{p-1, i}: S^{1} \rightarrow S^{1}$ be a monotone degree one map which we think of as a morphism $(p-1) \rightarrow(p)$ in $\Lambda$ such that $\phi_{p-1, i}\left(x_{p-1}^{k}\right)=x_{p}^{k}$ for $0 \leq k \leq i-1$, and $\phi_{p-1, i}\left(x_{p-1}^{k}\right)=x_{p}^{k+1}$ for $i \leq k \leq p-1$ (in particular, $x_{p}^{i}$ does not lie in the image of $\left.\left\{x_{p}^{k}\right\}_{0 \leq k \leq p-1}\right)$. Let $\mathbb{A}$ be an object of $\operatorname{Alg}_{\mathrm{As}}\left(\operatorname{Sp}^{\Sigma}(\mathbb{R})^{c}\right)$. Consider the composition $\Lambda^{\mathrm{op}} \simeq \operatorname{Disk}_{1}^{\dagger} /\left\langle S^{1}\right\rangle \rightarrow \widetilde{\mathrm{As}}^{\otimes} \rightarrow \mathrm{Sp}^{\Sigma}(\mathbb{R})^{c}$, where the final map is a symmetric monoidal functor corresponding to $\mathbb{A}$. By inspection, if $\left\{\phi_{p-1, i}\right\}_{0 \leq i \leq p}$ are regarded as morphisms $(p) \rightarrow(p-1)$ in $\Lambda^{\mathrm{op}}$, their images in $\operatorname{Sp}^{\Sigma}(\mathbb{R})^{c}$ define $(p+1)$ degeneracy maps $\mathbb{A}^{\wedge p+1} \rightarrow \mathbb{A}^{\wedge p}$ given by the multiplication $\mathbb{A} \wedge \mathbb{A} \rightarrow \mathbb{A}$. Let $\psi_{p, i}: S^{1} \rightarrow S^{1}$ be a monotone degree one map which we think of as a morphism $(p) \rightarrow(p-1)$ such that $\psi_{p, i}\left(x_{p}^{k}\right)=x_{p-1}^{k}$ for $k<i+1$, and $\psi_{p, i}\left(x_{p}^{k}\right)=x_{p-1}^{k-1}$ for $k \geq i+1$. As in the case of $\left\{\phi_{p-1, i}\right\}_{0 \leq i \leq p}$, these maps give rise to $p$ face maps $\mathbb{A}^{\wedge p} \rightarrow \mathbb{A}^{\wedge p+1}$ given by the unit $\mathbb{R} \rightarrow \mathbb{A}$. Consider the rotation $r_{p}: S^{1} \rightarrow S^{1}$ which sends $x_{p}^{k}$ to $x_{p}^{k+1}$ for $k \in \mathbf{Z} /(p+1) \mathbf{Z}$. We regard $r_{p}$ as an isomorphism $(p) \rightarrow(p)$. It yields the action of $\mathbf{Z} /(p+1) \mathbf{Z}$ on $\mathbb{A}^{\wedge p+1}$ given by the cyclic permutation of factors. It is straightforward to check that these maps constitute a cyclic object that coincides with the cyclic object obtained from $B \mathbb{A}$ in Definition 6.8.

Proof of Proposition 7.11. Taking into account Lemma 7.12 and $\operatorname{Sp}^{\Sigma}(\mathbb{R})^{c}\left[W^{-1}\right]^{\otimes} \simeq \operatorname{Mod}_{R}^{\otimes}$, for an $\mathbf{E}_{2}$-algebra $A$, we consider the image of $A$ under

$$
h: \operatorname{Alg}_{\mathbf{E}_{2}}\left(\operatorname{Mod}_{R}\right) \simeq \operatorname{Alg}_{\mathrm{As}}\left(\operatorname{Fun}^{\otimes}\left(\widetilde{\operatorname{As}}^{\otimes}, \operatorname{Mod}_{R}^{\otimes}\right)\right) \rightarrow \operatorname{Alg}_{\mathrm{As}}\left(\operatorname{Fun}\left(\Lambda^{\mathrm{op}}, \operatorname{Mod}_{R}\right)\right),
$$

where the right functor is induced by the composition with $\xi: \Lambda^{\mathrm{op}} \rightarrow \widetilde{\mathrm{As}}^{\otimes}$. We abuse notation by writing $\mathcal{H H}_{\bullet}^{\Lambda}(A)$ for the image of $A$ under $h$. In the following discussion, we will use the canonical identification $\operatorname{Alg}_{\mathbf{E}_{1}}^{\otimes}(-) \simeq \operatorname{Alg}_{\mathrm{As}_{\mathrm{s}}}^{\otimes}(-)$ which comes from the canonical equivalence $\mathbf{E}_{1}^{\otimes} \simeq \mathrm{As}^{\otimes}$ of $\infty$-operads. Let us consider

$$
\begin{aligned}
\operatorname{Alg}_{\mathbf{E}_{2}}\left(\operatorname{Mod}_{R}\right) & \simeq \operatorname{Alg}_{\mathbf{E}_{1}}\left(\operatorname{Alg}_{\mathrm{As}}^{\otimes}\left(\operatorname{Mod}_{R}\right)\right) \simeq \operatorname{Alg}_{\mathrm{As}}\left(\operatorname{Alg}_{\mathbf{E}_{1}}^{\otimes}\left(\operatorname{Mod}_{R}\right)\right) \\
& \leftarrow \operatorname{Fun}^{\otimes}\left(\widetilde{A s}^{\otimes}, \operatorname{Alg}_{\mathbf{E}_{1}}^{\otimes}\left(\operatorname{Mod}_{R}\right)\right) \rightarrow \operatorname{Fun}\left(\Lambda^{\mathrm{op}}, \operatorname{Alg}_{\mathbf{E}_{1}}\left(\operatorname{Mod}_{R}\right)\right) .
\end{aligned}
$$

The second equivalence follows from Construction 7.9, and the third functor is induced by $\xi$ : $\Lambda^{\mathrm{op}} \rightarrow \widetilde{\mathrm{As}}^{\otimes}$. The composition is identified with $h$ via the equivalence

$$
\operatorname{Alg}_{\mathbf{E}_{1}}\left(\operatorname{Fun}\left(\Lambda^{\mathrm{op}}, \operatorname{Mod}_{R}\right)\right) \simeq \operatorname{Fun}\left(\Lambda^{\mathrm{op}}, \operatorname{Alg}_{\mathbf{E}_{1}}\left(\operatorname{Mod}_{R}\right)\right)
$$

Let $A_{b}: \operatorname{As}^{\otimes} \rightarrow \operatorname{Alg}_{\mathbf{E}_{1}}^{\otimes}\left(\operatorname{Mod}_{R}\right)$ be a map of $\infty$-operads that corresponds to $A \in \operatorname{Alg}_{\mathbf{E}_{2}}\left(\operatorname{Mod}_{R}\right) \simeq$ $\operatorname{Alg}_{\text {As }}\left(\operatorname{Alg}_{\mathbf{E}_{1}}\left(\operatorname{Mod}_{R}^{\otimes}\right)\right)$. We let $\widetilde{A}_{b}: \widetilde{\mathrm{As}}^{\otimes} \rightarrow \operatorname{Alg}_{\mathbf{E}_{1}}^{\otimes}\left(\operatorname{Mod}_{R}\right)$ be a symmetric monoidal functor from the symmetric monoidal envelope $\widetilde{\mathrm{As}}^{\otimes}$ that corresponds to $A_{b}$ (namely, the composite $\mathrm{As}^{\otimes} \rightarrow \widetilde{\mathrm{As}}^{\otimes} \rightarrow \operatorname{Alg}_{\mathbf{E}_{1}}^{\otimes}\left(\operatorname{Mod}_{R}\right)$ is equivalent to $\left.A_{b}\right)$. Observe that the composite $\Lambda^{\text {op }} \stackrel{\xi}{\rightarrow} \widetilde{\mathrm{As}}^{\otimes} \stackrel{\widetilde{A}_{b}}{\rightarrow}$ $\operatorname{Alg}_{\mathbf{E}_{1}}^{\otimes}\left(\operatorname{Mod}_{R}\right)$ gives rise to a functor $\Lambda^{\text {op }} \rightarrow \operatorname{Alg}_{\mathbf{E}_{1}}\left(\operatorname{Mod}_{R}\right)$ which is equivalent to $\mathcal{H} \mathcal{H}_{\bullet}^{\Lambda}(A)$ in 
$\operatorname{Fun}\left(\Lambda^{\mathrm{op}}, \operatorname{Alg}_{\mathbf{E}_{1}}\left(\operatorname{Mod}_{R}\right)\right) \simeq \operatorname{Alg}_{\mathrm{As}}\left(\operatorname{Fun}\left(\Lambda^{\mathrm{op}}, \operatorname{Mod}_{R}\right)\right)$. Note that $\mathcal{H} \mathcal{H} \bullet(A)$ is defined to be the image of $\mathcal{H H}_{\bullet}^{\Lambda}(A)$ under the functor $\operatorname{Alg}_{\mathrm{As}}\left(\operatorname{Fun}\left(\Lambda^{\mathrm{op}}, \operatorname{Mod}_{R}\right)\right) \rightarrow \operatorname{Alg}_{\mathrm{As}}\left(\operatorname{Fun}\left(B S^{1}, \operatorname{Mod}_{R}\right)\right)$ induced by the symmetric monoidal functor $L: \operatorname{Fun}\left(\Lambda^{\mathrm{op}}, \operatorname{Mod}_{R}\right) \rightarrow \operatorname{Fun}\left(B S^{1}, \operatorname{Mod}_{R}\right)$ in Lemma 6.10. Here $L$ is a left adjoint of the symmetric monoidal functor Fun $\left(B S^{1}, \operatorname{Mod}_{R}\right) \rightarrow$ Fun $\left(\Lambda^{\mathrm{op}}, \operatorname{Mod}_{R}\right)$ induced by the composition with $\Lambda^{\mathrm{op}} \rightarrow B S^{1}$. Thus, $\mathcal{H} \mathcal{H} \bullet(A)$ can also be regarded as the image under the (left adjoint) functor $\operatorname{Fun}\left(\Lambda^{\mathrm{op}}, \operatorname{Alg}_{\mathbf{E}_{1}}\left(\operatorname{Mod}_{R}\right)\right) \rightarrow \operatorname{Fun}\left(B S^{1}\right.$, $\left.\operatorname{Alg}_{\mathbf{E}_{1}}\left(\operatorname{Mod}_{R}\right)\right)$ given by left Kan extensions along $\Lambda^{\mathrm{op}} \rightarrow B S^{1}$. Consequently, $\mathcal{H} \mathcal{H}_{\bullet}(A): B S^{1} \rightarrow$ $\operatorname{Alg}_{\mathbf{E}_{1}}\left(\operatorname{Mod}_{R}\right)$ is a left Kan extension of $\Lambda^{\mathrm{op}} \stackrel{\xi}{\rightarrow} \widetilde{\operatorname{As}} \stackrel{\otimes}{\widetilde{A}_{b}} \operatorname{Alg}_{\mathbf{E}_{1}}\left(\operatorname{Mod}_{R}\right)$ along $\Lambda^{\mathrm{op}} \rightarrow B S^{1}$. Next, we let $\widehat{A}_{b}: \mathbf{M f l d}_{1} \rightarrow \operatorname{Alg}_{\mathbf{E}_{1}}^{\otimes}\left(\operatorname{Mod}_{R}\right)$ be an operadic left Kan extension of $A_{b}: \mathbf{E}_{1}^{\otimes} \simeq$ $\mathrm{As}^{\otimes} \rightarrow \operatorname{Alg}_{\mathbf{E}_{1}}^{\otimes}\left(\operatorname{Mod}_{R}\right)$. Let $A_{b}^{\prime}: \operatorname{Mfld}_{1}^{\otimes} \rightarrow \operatorname{Alg}_{\mathbf{E}_{1}}^{\otimes}\left(\operatorname{Mod}_{R}\right)$ be a symmetric monoidal functor which corresponds to $\widehat{A}_{b}$. The composite $\operatorname{Disk}_{1}^{\otimes} \rightarrow \operatorname{Mfld}_{1}^{\otimes} \rightarrow \operatorname{Alg}_{\mathbf{E}_{1}}^{\otimes}\left(\operatorname{Mod}_{R}\right)$ is equivalent to $\widetilde{A}_{b}: \operatorname{Disk}_{1}^{\otimes} \simeq \widetilde{\mathrm{As}}^{\otimes} \rightarrow \operatorname{Alg}_{\mathbf{E}_{1}}^{\otimes}\left(\operatorname{Mod}_{R}\right)$. Consider the diagram of $\infty$-categories:

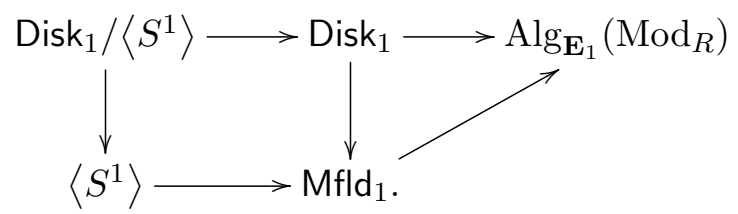

The upper left horizontal arrow is induced by the restriction to the source. The left vertical arrow is induced by the restriction to the target. The upper right arrow is the underlying functor of $\widetilde{A}_{b}$. The arrow $\mathrm{Mfld}_{1} \rightarrow \operatorname{Alg}_{\mathbf{E}_{1}}\left(\operatorname{Mod}_{R}\right)$ is the underlying functor of $A_{b}^{\prime}$. The right triangle commutes whereas the left square does not commute (but it admits a canonical natural transformation induced by the evaluation map $\left.\Delta^{1} \times \operatorname{Fun}\left(\Delta^{1}, \operatorname{Mfld}_{1}\right) \rightarrow \operatorname{Mfld}_{1}\right)$. The functor $\mathrm{Mfld}_{1} \rightarrow \operatorname{Alg}_{\mathbf{E}_{1}}\left(\operatorname{Mod}_{R}\right)$ carries $S^{1}$ to $\operatorname{colim}_{U \rightarrow S^{1} \in\left(\operatorname{Disk}_{1}\right)_{/ S^{1}}} \widetilde{A}_{b}(U)$ which means a colimit of $\mathbf{E}_{1}^{\otimes} \times_{\mathbf{M f l d}_{1}}\left(\mathbf{M f l d}_{1}^{\text {act }}\right)_{/ S^{1}} \simeq\left(\text { Disk }_{1}\right)_{/ S^{1}} \rightarrow$ Disk $_{1} \stackrel{\widetilde{A}_{b}}{\rightarrow} \operatorname{Alg}_{\mathbf{E}_{1}}\left(\operatorname{Mod}_{R}\right)$. By Lemma 7.6, the composite $\left\langle S^{1}\right\rangle \rightarrow \operatorname{Alg}_{\mathbf{E}_{1}}\left(\operatorname{Mod}_{R}\right)$ is a left Kan extension of $\Lambda^{\mathrm{op}} \simeq \operatorname{Disk}_{1}^{\dagger} /\left\langle S^{1}\right\rangle \rightarrow \operatorname{Alg}_{\mathbf{E}_{1}}\left(\operatorname{Mod}_{R}\right)$. Since $\Lambda^{\mathrm{op}} \simeq \operatorname{Disk}_{1}^{\dagger} /\left\langle S^{1}\right\rangle \rightarrow\left\langle S^{1}\right\rangle \simeq B S^{1}$ is a groupoid completion by Lemma 7.4 , it follows that the composite $B S^{1} \simeq\left\langle S^{1}\right\rangle \rightarrow \operatorname{Alg}_{\mathbf{E}_{1}}\left(\operatorname{Mod}_{R}\right)$ is equivalent to $\mathcal{H} \mathcal{H} \bullet(A)$. In other words, $\mathcal{H} \mathcal{H} \bullet(A)$ is equivalent to $B S^{1} \simeq\left\langle S^{1}\right\rangle \hookrightarrow \mathbf{M f l d}_{1} \stackrel{\widehat{A}_{b}}{\longrightarrow} \operatorname{Alg}_{\mathbf{E}_{1}}\left(\operatorname{Mod}_{R}\right)$.

Next, we relate $i_{!}(A)_{C}$ with $\mathcal{H} \mathcal{H}_{\bullet}(A): B S^{1} \stackrel{\iota}{\rightarrow} \mathbf{M f l d}_{1} \stackrel{\widehat{A}_{b}}{\rightarrow} \operatorname{Alg}_{\mathbf{E}_{1}}\left(\operatorname{Mod}_{R}\right)$. For this purpose, we consider the following setting. Let DCyl $\rightarrow \widetilde{\mathbf{D C y l}}$ be a symmetric monoidal envelope of DCyl. Composing with maps into symmetric monoidal envelopes, we have the left diagram
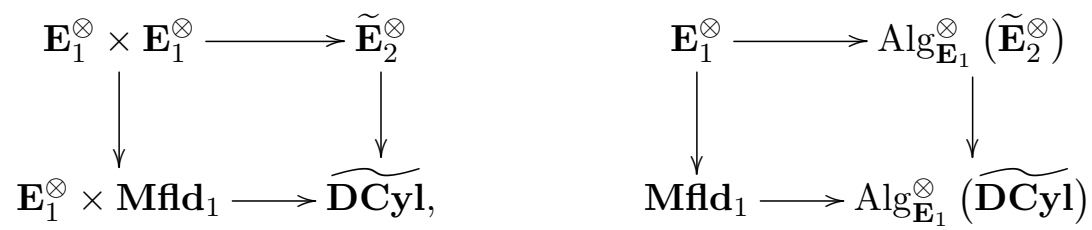

lying over $\wedge: \Gamma \times \Gamma \rightarrow \Gamma$. Then by the universal property of the tensor product of $\infty$-operads, it induces the right commutative diagram consisting of maps of $\infty$-operads over $\Gamma$, where $\operatorname{Alg}_{\mathbf{E}_{1}}^{\otimes}\left(\widetilde{\mathbf{E}}_{2}^{\otimes}\right)$ and $\operatorname{Alg}_{\mathbf{E}_{1}}^{\otimes}(\widetilde{\mathbf{D C y l}})$ are symmetric monoidal $\infty$-categories (defined over $\Gamma$ ), and the right vertical arrow is a symmetric monoidal (fully faithful) functor. In the following discussion, we replace $\operatorname{Mod}_{R}^{\otimes}$ by an arbitrary symmetric monoidal presentable $\infty$-category $\mathcal{M}^{\otimes}$ whose tensor product $\mathcal{M} \times \mathcal{M} \rightarrow \mathcal{M}$ preserves small colimits separately in each variable. The example of $\mathcal{M}^{\otimes}$ we keep in mind is $\operatorname{Mod}_{R}^{\otimes}$. Let $A$ be an $\mathbf{E}_{2}$-algebra object in $\mathcal{M}^{\otimes}$, that is, a map $A: \mathbf{E}_{2}^{\otimes} \rightarrow \mathcal{M}^{\otimes}$ of $\infty$-operads over $\Gamma$. The inclusion $i: \mathbf{E}_{2}^{\otimes} \hookrightarrow \mathbf{D C y l}$ gives rise to the 
adjoint pair $i_{!}: \operatorname{Alg}_{\mathbf{E}_{2}}(\mathcal{M}) \rightleftarrows \operatorname{Alg}_{\mathbf{D C y l}}(\mathcal{M}): i^{*}$. Let $\widetilde{A}: \widetilde{\mathbf{E}}_{2}^{\otimes} \rightarrow \mathcal{M}^{\otimes}$ and $\widetilde{i_{!}(A)}: \widetilde{\mathbf{D C y l}} \rightarrow \mathcal{M}^{\otimes}$ be symmetric monoidal functors that correspond to $A$ and $i_{!}(A)$, respectively. We have the diagram of $\infty$-operads

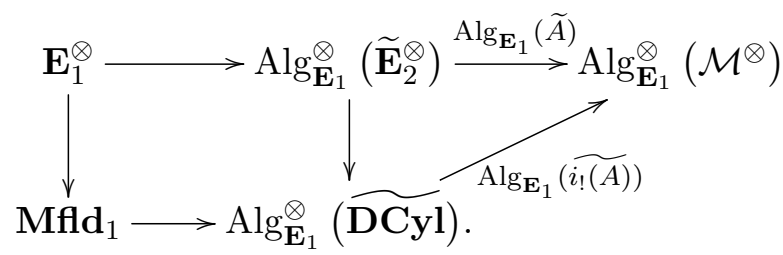

As before, we let $A_{b}: \mathbf{E}_{1}^{\otimes} \rightarrow \operatorname{Alg}_{\mathbf{E}_{1}}\left(\mathcal{M}^{\otimes}\right)$ be the composite of top horizontal arrows, which amounts to $A: \mathbf{E}_{2}^{\otimes} \rightarrow \mathcal{M}^{\otimes}$ since $\mathbf{E}_{1}^{\otimes} \otimes \mathbf{E}_{1}^{\otimes} \simeq \mathbf{E}_{2}^{\otimes}$. Let $\hat{A}_{b}: \operatorname{Mfld}_{1} \rightarrow \operatorname{Alg}_{\mathbf{E}_{1}}^{\otimes}\left(\mathcal{M}^{\otimes}\right)$ be the operadic left Kan extension of $A_{b}$ along $\mathbf{E}_{1}^{\otimes} \hookrightarrow \mathbf{M f l d}_{1}$. Let $\hat{A}_{\sharp}: \mathbf{M f l d}_{1} \rightarrow \operatorname{Alg}_{\mathbf{E}_{1}}^{\otimes}\left(\mathcal{M}^{\otimes}\right)$ be the composite. We note that $i_{!}(A)_{C}$ is equivalent to $B S^{1} \rightarrow \operatorname{Alg}_{\mathbf{E}_{1}}\left(\mathcal{M}^{\otimes}\right)$ determined by the composite $B S^{1} \simeq\left\langle S^{1}\right\rangle \stackrel{\iota}{\hookrightarrow} \mathbf{M f l d}_{1} \stackrel{\hat{A}_{\sharp}}{\rightarrow} \operatorname{Alg}_{\mathbf{E}_{1}}^{\otimes}\left(\mathcal{M}^{\otimes}\right)$. The universal property [25, Proposition 3.1.3.2] induces a canonical morphism $\hat{A}_{b} \rightarrow \hat{A}_{\sharp}$. It suffices to prove that the restriction $\left.\left.\hat{A}_{b}\right|_{\left\langle S^{1}\right\rangle} \rightarrow \hat{A}_{\sharp}\right|_{\left\langle S^{1}\right\rangle}$ to $\left\langle S^{1}\right\rangle$ is an equivalence. (It gives rise to an equivalence $\mathcal{H} \mathcal{H} \bullet(A) \simeq i_{!}(A)_{C}$ in Fun $\left(B S^{1}, \operatorname{Alg}_{\mathbf{E}_{1}}\left(\operatorname{Mod}_{R}\right)\right)$.) To this end, it is enough to show the following lemma, which completes the proof of Proposition 7.11.

Lemma 7.13. The induced morphism $\hat{A}_{b}\left(S^{1}\right) \rightarrow \hat{A}_{\sharp}\left(S^{1}\right)$ is an equivalence in the $\infty$-category $\operatorname{Alg}_{\mathbf{E}_{1}}\left(\mathcal{M}^{\otimes}\right)$.

Proof. We first consider $\hat{A}_{b}: \mathbf{M f l d}_{1} \rightarrow \operatorname{Alg}_{\mathbf{E}_{1}}^{\otimes}\left(\mathcal{M}^{\otimes}\right)$. The operadic left Kan extension gives $\hat{A}_{b}\left(S^{1}\right)=\operatorname{colim}_{U \rightarrow S^{1} \in\left(\text { Disk }_{1}\right)_{/ S^{1}}} \widetilde{A}_{b}(U)$. Here, $\operatorname{colim}_{U \rightarrow S^{1} \in\left(\operatorname{Disk}_{1}\right)_{/ S^{1}}} \widetilde{A}_{b}(U)$ means a colimit of $\mathbf{E}_{1}^{\otimes} \times$ $\mathbf{M f l d}_{1}\left(\mathbf{M f l d}_{1}\right)_{/ S^{1}}^{\text {act }} \simeq\left(\right.$ Disk $\left._{1}\right) / S^{1} \rightarrow$ Disk $_{1} \stackrel{\widetilde{A}_{2}}{\rightarrow} \operatorname{Alg}_{\mathbf{E}_{1}}\left(\mathcal{M}^{\otimes}\right)$. By [2, Corollary 3.22] or [25, Proposition 5.5.2.15], $\left(\right.$ Disk $\left._{1}\right) / S^{1}$ is sifted (strictly speaking, in the statement in [25, Proposition 5.5.2.15], mapping spaces between disks are spaces of (not necessarily rectilinear) open embeddings, but the overcategory $\left(\right.$ Disk $\left._{1}\right) / S^{1}$ is equivalent to a nonrectilinear version in loc. cit.). The forgetful functor $\operatorname{Alg}_{\mathbf{E}_{1}}\left(\mathcal{M}^{\otimes}\right) \rightarrow \mathcal{M}$ preserves sifted colimits. Consequently, the image of $\hat{A}_{b}\left(S^{1}\right)$ in $\mathcal{M}$ is a colimit of $\left(\text { Disk }_{1}\right)_{/ S^{1}} \rightarrow \operatorname{Alg}_{\mathbf{E}_{1}}\left(\mathcal{M}^{\otimes}\right) \rightarrow \mathcal{M}$. Given a topological $r$-manifold $T$, we let $\operatorname{Disj}(T)$ be the poset that consists of open sets $U \subset T$ such that $U$ is homeomorphic to a finite disjoint union of $(0,1)^{r}$. We think of $\operatorname{Disj}(T)$ as a category. Then according to [25, Proposition 5.5.2.13], the natural functor $\operatorname{Disj}\left(S^{1}\right) \rightarrow\left(\operatorname{Disk}_{1}\right) / S^{1}$ is left cofinal. Thus, $\operatorname{colim}_{U \in \operatorname{Disj}\left(S^{1}\right)} \widetilde{A}_{b}(U) \simeq \operatorname{colim}_{U \rightarrow S^{1} \in\left(\operatorname{Disk}_{1}\right)_{/ S^{1}}} \widetilde{A}_{b}(U)$ in $\mathcal{M}$, where $\operatorname{colim}_{U \in \operatorname{Disj}\left(S^{1}\right)} A_{b}(U)$ is a colimit of $\operatorname{Disj}\left(S^{1}\right) \rightarrow\left(\text { Disk }_{1}\right)_{/ S^{1}} \rightarrow \mathcal{M}$ (this equivalence also follows from the fact that (Disk $)_{1} / S^{1}$ is obtained from $\operatorname{Disj}\left(S^{1}\right)$ by localizing with respect to those inclusions $U \subset U^{\prime}$ that are isotopic to an isomorphism [2, Proposition 2.19]).

Next, we consider the image of $\hat{A}_{\sharp}\left(S^{1}\right)$ in $\mathcal{M}$. If Disk 2 denotes the underlying $\infty$-category of Disk $_{2}^{\otimes}$ and we set $C=(0,1) \times S^{1}$, then the image of $C$ in $\mathcal{M}$ under the operadic left Kan extension $i_{!}(A)$ is $\operatorname{colim}_{V \rightarrow C \in\left(\text { Disk }_{2}\right)_{C}} A(V)$, that is, a colimit of $\mathbf{E}_{2}^{\otimes} \times{ }_{\text {DCyl }}(\mathbf{D C y l})_{/ C}^{\text {act }} \simeq$ $\left(\text { Disk }_{2}\right)_{/ C} \rightarrow$ Disk $_{2} \stackrel{\widetilde{A}}{\rightarrow} \mathcal{M}$, where the latter functor is the underlying functor of the induced symmetric monoidal functor $\widetilde{A}$ : Disk ${ }_{2}^{\otimes} \rightarrow \mathcal{M}^{\otimes}$ (we abuse notation).

Let $\operatorname{Disj}^{\text {rec }}(C)$ be the full subcategory (poset) of $\operatorname{Disj}(C)$ spanned by those open sets $V \subset C$ such that $V$ is the image of a rectilinear embedding, and the composite $V \hookrightarrow C=(0,1) \times S^{1}$ $\stackrel{\mathrm{pr}}{\rightarrow} S^{1}$ is not surjective. By applying the argument of [25, Proposition 5.5.2.13] to $\operatorname{Disj}^{\mathrm{rec}}(C) \rightarrow$ 
$\left(\text { Disk }_{2}\right)_{/ C}$, we see that $\operatorname{Disj}^{\text {rec }}(C) \rightarrow\left(\text { Disk }_{2}\right)_{/ C}$ is left cofinal. It follows that there is a canonical equivalence $\operatorname{colim}_{V \in \operatorname{Disj}^{\mathrm{rec}}(C)} \widetilde{A}(V) \simeq \operatorname{colim}_{V \rightarrow C \in\left(\text { Disk }_{2}\right)_{/ C}} \widetilde{A}(V)$.

To prove that $\hat{A}_{b}\left(S^{1}\right) \rightarrow \hat{A}_{\sharp}\left(S^{1}\right)$ is an equivalence, it is enough to show that

$$
\operatorname{colim}_{U \in \operatorname{Disj}\left(S^{1}\right)} \widetilde{A}_{b}(U) \rightarrow \operatorname{colim}_{V \in \operatorname{Disj}^{\mathrm{rec}}(C)} \widetilde{A}(V)
$$

is an equivalence in $\mathcal{M}$. Unwinding the definition, this morphism is the composite of

$$
\operatorname{colim}_{U \in \operatorname{Disj}\left(S^{1}\right)} \widetilde{A}_{b}(U) \rightarrow \operatorname{colim}_{(0,1) \times U \in \operatorname{Disj}^{\mathrm{rec}}(C)} \widetilde{A}((0,1) \times U) \rightarrow \operatorname{colim}_{V \in \operatorname{Disj}^{\mathrm{rec}}(C)} \widetilde{A}(V),
$$

where the right arrow is induced by the universal property of the colimit, and the left arrow is an equivalence because $\widetilde{A}_{b}(U) \simeq \widetilde{A}((0,1) \times U)$. To see that the right arrow is an equivalence, it will suffice to prove that $\operatorname{Disj}\left(S^{1}\right) \rightarrow \operatorname{Disj}^{\mathrm{rec}}(C)$ that sends $U$ to $(0,1) \times U$ is left cofinal: for any $V \in \operatorname{Disj}^{\text {rec }}(C)$, the category $\operatorname{Disj}\left(S^{1}\right) \times{ }_{\operatorname{Disi}}{ }^{r e c}(C) \operatorname{Disj}^{\text {rec }}(C)_{V /}$ is weakly contractible. Consider the image $W$ of $V$ under the projection $(0,1) \times S^{1} \rightarrow S^{1}$. Then $W$ belongs to $\operatorname{Disj}\left(S^{1}\right)$ since $V \hookrightarrow C \rightarrow S^{1}$ is not surjective. It follows that $\operatorname{Disj}\left(S^{1}\right) \times_{\operatorname{Disj}^{\mathrm{rec}}(C)} \operatorname{Disj}^{\mathrm{rec}}(C)_{V /}$ has an initial object so that the opposite category is filtered. Thus, by [27, Proposition 5.5.8.7], $\operatorname{Disj}\left(S^{1}\right) \times_{\operatorname{Disj}^{\mathrm{rec}}(C)} \operatorname{Disj}^{\mathrm{rec}}(C)_{V /}$ is weakly contractible as desired.

Theorem 7.14. Let $R$ be a commutative ring spectrum. Let $\mathcal{C}$ be a small $R$-linear stable idempotent-complete $\infty$-category, that is, an object of $\mathcal{S} \mathrm{t}_{R}=\operatorname{Mod}_{\operatorname{Perf}_{R}}(\mathcal{S} \mathrm{t})$. Let $\mathcal{H} \mathcal{H}(\mathcal{C})$ be the Hochschild cohomology $R$-module spectrum which belongs to $\operatorname{Alg}_{\mathbf{E}_{2}}\left(\operatorname{Mod}_{R}\right)$, see Definitions 5.3 and 5.4 . Let $\mathcal{H} \mathcal{H}_{\bullet}(\mathcal{C})$ be the Hochschild homology $R$-module spectrum which lies in $\operatorname{Fun}\left(B S^{1}, \operatorname{Mod}_{R}\right)$, see Definition 6.14. Then $\left(\mathcal{H} \mathcal{H}^{\bullet}(\mathcal{C}), \mathcal{H} \mathcal{H} \bullet(\mathcal{C})\right)$ is promoted to an object of $\operatorname{Alg}_{\mathbf{K S}}\left(\operatorname{Mod}_{R}\right)$ in a natural way.

Remark 7.15. By Corollary 4.21, we have

$$
\begin{aligned}
\operatorname{Alg}_{\mathbf{K S}}\left(\operatorname{Mod}_{R}\right) & \simeq \operatorname{Alg}_{\mathbf{E}_{2}}\left(\operatorname{Mod}_{R}\right) \times \operatorname{Alg}_{\mathbf{E}_{1}}\left(\operatorname{Fun}\left(B S^{1}, \operatorname{Mod}_{R}\right)\right) \\
& \rightarrow \operatorname{Alg}_{\mathbf{E}_{2}}\left(\operatorname{Mod}_{R}\right) \times \operatorname{Fun}\left(B S^{1}, \operatorname{Mod}_{R}\right) .
\end{aligned}
$$

Theorem 7.14 means that we can obtain an object of $\operatorname{Alg}_{\mathbf{K S}}\left(\operatorname{Mod}_{R}\right)$ which "lies over" the pair $\left(\mathcal{H} \mathcal{H}^{\bullet}(\mathcal{C}), \mathcal{H} \mathcal{H}_{\bullet}(\mathcal{C})\right) \in \operatorname{Alg}_{\mathbf{E}_{2}}\left(\operatorname{Mod}_{R}\right) \times \operatorname{Fun}\left(B S^{1}, \operatorname{Mod}_{R}\right)$.

The proof proceeds in Construction 7.16, Proposition 7.17 and Construction 7.18.

Construction 7.16. We write $\mathcal{D}$ for the Ind-category $\operatorname{Ind}(\mathcal{C})$, which is an $R$-linear compactly generated stable $\infty$-category. Let $\mathcal{H H}^{\bullet}(\mathcal{C})=\mathcal{H} \mathcal{H}^{\bullet}(\mathcal{D})$ be the Hochschild cohomology $R$-module spectrum. Recall that $\mathcal{H H}^{\bullet}(\mathcal{D})=E\left(\mathcal{E} \operatorname{nd}_{R}(\mathcal{D})\right)$. The counit map of the adjunction $I: \operatorname{Alg}_{\mathbf{E}_{2}}\left(\operatorname{Mod}_{R}\right) \rightleftarrows \operatorname{Alg}_{\mathrm{As}}\left(\operatorname{Pr}_{R}^{\mathrm{L}}\right): E$ induces to an associative monoidal functor

$$
\operatorname{RMod}_{\mathcal{H} \mathcal{H}^{\bullet}(\mathcal{C})}^{\otimes}=\operatorname{RMod}_{\mathcal{H} \mathcal{H}^{\bullet}(\mathcal{D})}^{\otimes} \rightarrow \mathcal{E} \operatorname{nd}_{R}^{\otimes}(\mathcal{D}),
$$

that is, a morphism in $\operatorname{Alg}_{\mathrm{As}}\left(\operatorname{Pr}_{R}^{\mathrm{L}}\right)$, where $\operatorname{RMod}_{\mathcal{H} \mathcal{H}^{\bullet}(\mathcal{C})}^{\otimes}:=\operatorname{RMod}_{\mathcal{H} \mathcal{H}^{\bullet}(\mathcal{C})}^{\otimes}\left(\operatorname{Mod}_{R}\right)$ is the associative monoidal $\infty$-category of right modules of $\mathcal{H} \mathcal{H}^{\bullet}(\mathcal{C})$. According to [25, Corollary 4.7.1.40], the associative monoidal $\infty$-category $\mathcal{E} \operatorname{nd}_{R}^{\otimes}(\mathcal{D})$ naturally acts on $\mathcal{D}$. More precisely, $\mathcal{D}$ is a left module of $\mathcal{E} \mathrm{nd}_{R}^{\otimes}(\mathcal{D})$, that is, an object of $\operatorname{LMod}_{\mathcal{E n d}_{R}^{\otimes}(\mathcal{D})}\left(\operatorname{Pr}_{R}^{\mathrm{L}}\right)$ (this action is universal in an appropriate sense, cf. [25, Section 4.7.1]). Then the associative monoidal functor $\operatorname{RMod}_{\mathcal{H} \mathcal{H}^{\bullet}(\mathcal{C})}^{\otimes} \rightarrow \mathcal{E n d}_{R}^{\otimes}(\mathcal{D})$ induces a left $\operatorname{RMod}_{\mathcal{H} \mathcal{H}^{\bullet}(\mathcal{C})}^{\otimes}$-module structure on $\mathcal{D}$. That is, $\mathcal{D}$ is promoted to an object of $\operatorname{LMod}_{\mathrm{RMod}}{ }_{\mathcal{H} \mathcal{H}^{\bullet}(\mathcal{C})}\left(\operatorname{Pr}_{R}^{\mathrm{L}}\right)$.

Let $\operatorname{RPerf}_{\mathcal{H} \mathcal{H}^{\bullet}(\mathcal{C})}$ be the full subcategory of $\operatorname{RMod}_{\mathcal{H}} \bullet(\mathcal{C})$ spanned by compact objects. This subcategory is the smallest stable subcategory which contains $\mathcal{H} \mathcal{H} \bullet(\mathcal{C})$ (regarded as a right 
module) and is closed under retracts. Hence $\operatorname{RPerf}_{\mathcal{H} \mathcal{H}^{\bullet}(\mathcal{C})}$ inherits an associative monoidal structure from the structure on $\operatorname{RMod}_{\mathcal{H} \mathcal{H}^{\bullet}(\mathcal{C})}$. We denote by $\operatorname{RPerf}_{\mathcal{H} \mathcal{H}^{\bullet}(\mathcal{C})}^{\otimes}$ the resulting associative monoidal small $R$-linear stable idempotent-complete $\infty$-category which we regard as an object of $\operatorname{Alg}_{\mathrm{As}}\left(\mathcal{S} \mathrm{t}_{R}\right)$.

Proposition 7.17. We continue to assume that $\mathcal{C}$ is a small $R$-linear stable idempotent-complete $\infty$-category. If we think of $\mathcal{D}=\operatorname{Ind}(\mathcal{C})$ as the left $\operatorname{RMod}_{\mathcal{H} \mathcal{H}^{\bullet}(\mathcal{C})}^{\otimes}$-module (as above), the restriction exhibits $\mathcal{C}$ as a left $\operatorname{RPerf}_{\mathcal{H} \mathcal{H}^{\bullet}(\mathcal{C})}^{\otimes}$-module, that is, an object of $\operatorname{LMod}_{\operatorname{RPerf}_{\mathcal{H} \mathcal{H}^{\bullet}(\mathcal{C})}^{\otimes}}\left(\mathcal{S} \mathrm{t}_{R}\right)$. In particular, $\mathcal{C}$ is promoted to an object of $\operatorname{LMod}_{\mathrm{RPerf}}^{\otimes} \mathcal{H \mathcal { H }}_{(\mathcal{C})}\left(\mathcal{S} \mathrm{t}_{R}\right)$.

Proof. We may and will suppose that $\mathcal{C}$ is the full subcategory of compact objects in $\mathcal{D}$. The tensor product functor

$$
\operatorname{RMod}_{\mathcal{H} \mathcal{H}^{\bullet}(\mathcal{C})} \times \operatorname{RMod}_{\mathcal{H} \mathcal{H}^{\bullet}(\mathcal{C})} \rightarrow \operatorname{RMod}_{\mathcal{H} \mathcal{H}^{\bullet}(\mathcal{C})}
$$

sends $\operatorname{RPerf}_{\mathcal{H} \mathcal{H}^{\bullet}(\mathcal{C})} \times \operatorname{RPerf}_{\mathcal{H} \mathcal{H}^{\bullet}(\mathcal{C})}$ to $\operatorname{RPerf}_{\mathcal{H} \mathcal{H}^{\bullet}(\mathcal{C})} \subset \operatorname{RMod}_{\mathcal{H} \mathcal{H}^{\bullet}(\mathcal{C})}$. It will suffice to prove that the action functor

$$
m: \operatorname{RMod}_{\mathcal{H} \mathcal{H}^{\bullet}(\mathcal{C})} \times \mathcal{D} \rightarrow \mathcal{D}
$$

sends $\operatorname{RPerf}_{\mathcal{H} \mathcal{H}}{ }^{(\mathcal{C})} \times \mathcal{C}$ to $\mathcal{C}$. Let $\mathcal{P}$ be the full subcategory of $\operatorname{RMod}_{\mathcal{H}} \bullet(\mathcal{C})$ spanned by those objects $P$ such that the essential image of $\{P\} \times \mathcal{C}$ is contained in $\mathcal{C}$. Note that $m$ preserves the shift functors $(\Sigma$ or $\Omega$ ) and small colimits separately in each variable. Moreover, the stable subcategory $\mathcal{C} \subset \mathcal{D}$ is closed under retracts. Thus, we see that $\mathcal{P}$ is a stable subcategory which is closed under retracts. Since $\mathcal{H H}^{\bullet}(\mathcal{C})$ is a unit object, $\mathcal{H H}^{\bullet}(\mathcal{C})$ lies in $\mathcal{P}$. Keep in mind that $\operatorname{RPerf}_{\mathcal{H} \mathcal{H}^{\bullet}(\mathcal{C})}$ is the smallest stable subcategory which contains $\mathcal{H H}^{\bullet}(\mathcal{C})$ and is closed under retracts. It follows that $\operatorname{RPerf}_{\mathcal{H} \mathcal{H}^{\bullet}(\mathcal{C})} \subset \mathcal{P}$.

Construction 7.18. Take $\mathcal{O}$ to be $\mathcal{L} \mathcal{M}$ in Proposition 6.15. By definition, $\operatorname{LMod}\left(\operatorname{Mod}_{\operatorname{Perf}_{R}}(\mathcal{S t})\right)$ is $\operatorname{Alg}_{\mathcal{L M}}\left(\operatorname{Mod}_{\operatorname{Perf}_{R}}(\mathcal{S t})\right)$, and $\operatorname{LMod}\left(\operatorname{Fun}\left(B S^{1}, \operatorname{Mod}_{R}\right)\right)=\operatorname{Alg} \log _{\mathcal{L} \mathcal{M}}\left(\operatorname{Fun}\left(B S^{1}, \operatorname{Mod}_{R}\right)\right)$. We then have

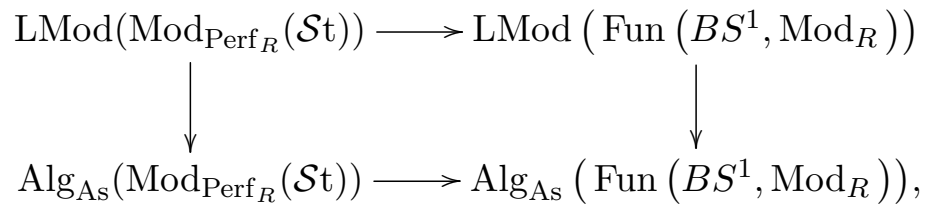

where the vertical arrows are given by the restriction along $A s^{\otimes} \hookrightarrow \mathcal{L} \mathcal{M}$. By Proposition 7.17, we think of $\mathcal{C}$ as an object of $\operatorname{LMod}_{\mathrm{RPerf}_{\mathcal{H} \mathcal{H}}^{\bullet}(\mathcal{C})}^{\otimes}\left(\operatorname{Mod}_{\operatorname{Perf}_{R}}(\mathcal{S t})\right)$. Applying the above functor to $\mathcal{C}$, we obtain $\mathcal{H}_{\bullet}(\mathcal{C})$ which belongs to $\operatorname{LMod}_{\mathcal{H} \mathcal{H}_{\bullet}\left(\operatorname{RPerf}_{\mathcal{H}} \bullet(\mathcal{C})\right.}\left(\operatorname{Fun}\left(B S^{1}, \operatorname{Mod}_{R}\right)\right)$. The lower horizontal arrow carries the associative monoidal $\infty$-category $\operatorname{RPerf}_{\mathcal{H} \mathcal{H} \bullet(\mathcal{C})}^{\otimes}$ to an object of $\operatorname{Alg}_{\text {As }}\left(\operatorname{Fun}\left(B S^{1}, \operatorname{Mod}_{R}\right)\right)$. That is, $\mathcal{H H}_{\bullet}\left(\operatorname{RPerf}_{\mathcal{H} \mathcal{H}} \bullet \mathcal{C}\right)$ is an associative algebra object in Fun $\left(B S^{1}, \operatorname{Mod}_{R}\right)$. Consequently, the Hochschild homology $R$-module spectrum $\mathcal{H} \mathcal{H} \cdot(\mathcal{C})$ is a left $\mathcal{H} \mathcal{H}_{\bullet}\left(\operatorname{RPerf}_{\mathcal{H} \mathcal{H}^{\bullet}(\mathcal{C})}\right)$-module object in $\operatorname{Fun}\left(B S^{1}, \operatorname{Mod}_{R}\right)$. Next, we set $A=\mathcal{H H}^{\bullet}(\mathcal{C})$ in $\operatorname{Alg}_{\mathbf{E}_{2}}\left(\operatorname{Mod}_{R}\right)$. By the invariance of Hochschild homology under Morita equivalences (cf. Lemma 6.11), $\mathcal{H H}_{\bullet}(A) \simeq \mathcal{H}_{\bullet}\left(\operatorname{RPerf}_{\mathcal{H} \mathcal{H}^{\bullet}(\mathcal{C})}\right)$ in $\operatorname{Alg}_{\mathrm{As}}\left(\operatorname{Fun}\left(B S^{1}, \operatorname{Mod}_{R}\right)\right)$. Let

$$
\operatorname{Alg}_{\mathbf{E}_{2}}\left(\operatorname{Mod}_{R}\right) \stackrel{i !}{\simeq} \operatorname{Alg}_{\mathbf{D C y l}}^{\mathbf{D}}\left(\operatorname{Mod}_{R}\right) \rightarrow \operatorname{Alg}_{\mathbf{C y l}}\left(\operatorname{Mod}_{R}\right) \simeq \operatorname{Alg}_{\mathbf{E}_{1}}\left(\operatorname{Fun}\left(B S^{1}, \operatorname{Mod}_{R}\right)\right)
$$

be a sequence of functors such that the first one is induced by left Kan extensions along $i: \mathbf{E}_{2}^{\otimes} \hookrightarrow$ DCyl (cf. the discussion before Proposition 4.19), the second one is the restriction along Cyl $\rightarrow$ DCyl, and the third functor (equivalence) comes from Corollary 4.18. 
By definition, the image of $A$ in $\operatorname{Alg}_{\mathbf{E}_{1}}\left(\operatorname{Fun}\left(B S^{1}, \operatorname{Mod}_{R}\right)\right)$ is $i_{!}(A)_{C}$ defined in the discussion before Proposition 7.11. According to Proposition 7.11, we have the canonical equivalence $\mathcal{H} \mathcal{H} \bullet(A) \simeq i_{!}(A)_{C}$ in $\operatorname{Alg}_{\mathbf{E}_{1}}\left(\operatorname{Fun}\left(B S^{1}, \operatorname{Mod}_{R}\right)\right) \simeq \operatorname{Alg}_{\mathrm{As}}\left(\operatorname{Fun}\left(B S^{1}, \operatorname{Mod}_{R}\right)\right)$. Therefore, $\mathcal{H H}^{\bullet}(\mathcal{C})=A \in \operatorname{Alg}_{\mathbf{E}_{2}}\left(\operatorname{Mod}_{R}\right)$ and the left $\mathcal{H} \mathcal{H} \bullet(A)$-module $\mathcal{H} \mathcal{H} \bullet(\mathcal{C})$ together with $i_{!}(A)_{C} \simeq$ $\mathcal{H} \mathcal{H} \bullet(A)$ determines an object of

$$
\operatorname{Alg}_{\mathbf{E}_{2}}\left(\operatorname{Mod}_{R}\right) \times{ }_{\operatorname{Alg}_{\text {As }}\left(\operatorname{Fun}\left(B S^{1}, \operatorname{Mod}_{R}\right)\right)} \operatorname{LMod}\left(\operatorname{Fun}\left(B S^{1}, \operatorname{Mod}_{R}\right)\right) \simeq \operatorname{Alg}_{\mathbf{K S}}\left(\operatorname{Mod}_{R}\right),
$$

where the equivalence comes from the canonical equivalences in Corollary 4.21. In other words, it defines an object of $\operatorname{Alg} \frac{\mathbf{D}}{\mathbf{D C y l}}\left(\operatorname{Mod}_{R}\right) \subset \operatorname{Alg} \frac{}{\overline{\mathbf{D C y l}}}\left(\operatorname{Mod}_{R}\right)$ which induces a KS-algebra via the restriction. Thus, we obtain the desired object of $\operatorname{Alg}_{\mathbf{K S}}\left(\operatorname{Mod}_{R}\right)$.

\section{The action}

In this section, we study the maps induced by the action of Hochschild cohomology spectrum $\mathcal{H} \mathcal{H}^{\bullet}(\mathcal{C})$ on $\mathcal{H} \mathcal{H} \bullet(\mathcal{C})$, constructed in Theorem 7.14 .

8.1. Let $R$ be a commutative ring spectrum. We let $\mathcal{C}$ be a small stable $R$-linear $\infty$-category. We let $A \in \operatorname{Alg}_{\text {As }}\left(\operatorname{Mod}_{R}\right)$ and suppose that $\mathcal{C}=\operatorname{RPerf}_{A}$. In other words, we assume that $\operatorname{Ind}(\mathcal{C})$ admits a single compact generator. In this setting, we can describe morphisms induced by module actions by means of concrete algebraic constructions. For ease of notation, we write $\mathcal{H} \mathcal{H}^{\bullet}(A)$ for $\mathcal{H H}^{\bullet}\left(\operatorname{RPerf}_{A}\right)=\mathcal{H H}^{\bullet}\left(\operatorname{RMod}_{A}\right)$. We can safely confuse $\mathcal{H} \mathcal{H} \bullet\left(\operatorname{RMod}_{A}\right)$ with the Hochschild homology $R$-module spectrum $\mathcal{H H}_{\bullet}(A)$ of $A$ because of the invariance under Morita equivalences. We do not distinguish between the notation $\mathcal{H} \mathcal{H} \bullet\left(\operatorname{RMod}_{A}\right)$ and $\mathcal{H} \mathcal{H} \bullet(A)$ : we write $\mathcal{H} \mathcal{H}_{\bullet}(A)$ for $\mathcal{H}_{\bullet}\left(\operatorname{RMod}_{A}\right)$ as well. Write $A^{e}$ for $A^{\text {op }} \otimes_{R} A$. As before, by $\otimes$ we mean the tensor product over $R$ when we treat the tensor products of objects in $\operatorname{Mod}_{R}$ or $\operatorname{Alg}_{\mathrm{As}}\left(\operatorname{Mod}_{R}\right)$.

We define a morphism $\mathcal{H H}^{\bullet}(A) \otimes \mathcal{H} \mathcal{H}_{\bullet}(A) \rightarrow \mathcal{H H}_{\bullet}(A)$ which we refer to as the contraction morphism:

Definition 8.1. Consider the functor

$$
(-) \otimes_{A^{e}}(-): \operatorname{RMod}_{A^{e}} \times \operatorname{LMod}_{A^{e}} \rightarrow \operatorname{Mod}_{R}
$$

which is informally given by the two-sided bar construction $(P, Q) \mapsto P \otimes_{A^{e}} Q$. Note that $\operatorname{RMod}_{A^{e}}$ is left-tensored over $\operatorname{Mod}_{R}^{\otimes}$. If we regard $\mathcal{H H}^{\bullet}(A)$ as an object of $\operatorname{Mod}_{R}$, there is a morphism $\mathcal{H H}^{\bullet}(A) \otimes A \rightarrow A$ in $\operatorname{RMod}_{A^{e}}$, which exhibits $\mathcal{H H}^{\bullet}(A)$ as a morphism object from $A$ to itself in $\operatorname{RMod}_{A^{e}}$ (i.e., hom $R$-module), see Corollary 8.6. Let $\left(\mathcal{H} \mathcal{H}^{\bullet}(A) \otimes A\right) \otimes_{A^{e}} A \rightarrow A \otimes_{A^{e}} A$ be the morphism induced by the morphism $\mathcal{H H}^{\bullet}(A) \otimes A \rightarrow A$ in $\operatorname{RMod}_{A^{e}}$. We identify $\mathcal{H} \mathcal{H} \bullet(A)$ with $A \otimes_{A^{e}} A$ in $\operatorname{Mod}_{R}$, see Lemma 8.7. It gives rise to

$$
\sigma: \mathcal{H H}^{\bullet}(A) \otimes \mathcal{H H}_{\bullet}(A)=\mathcal{H H}^{\bullet}(A) \otimes\left(A \otimes_{A^{e}} A\right) \rightarrow A \otimes_{A^{e}} A=\mathcal{H} \mathcal{H} \bullet(A) .
$$

We shall refer to it as the contraction morphism.

We denote by $\left(\mathcal{H}^{\bullet}(\mathcal{C}), \mathcal{H} \mathcal{H}_{\bullet}(\mathcal{C})\right)$ the pair endowed with the $\mathbf{K S}$-algebra structure constructed in Theorem 7.14: we will think that the pair is promoted to an object of $\operatorname{Alg}_{\mathbf{K S}}\left(\operatorname{Mod}_{R}\right)$. Let $D$ and $C_{M}$ be colors in the colored operad KS. There is a class of an active morphism $f_{j}:\left(\langle 2\rangle, D, C_{M}\right) \rightarrow\left(\langle 1\rangle, C_{M}\right)$ in $\mathbf{K S}$ lying over the active morphism $\rho:\langle 2\rangle \rightarrow\langle 1\rangle\left(\right.$ with $\left.\rho^{-1}(*)=*\right)$. Such a morphism $f_{j}$ is unique up to equivalences. This is induced by an open embedding $j:(0,1)^{2} \sqcup(0,1) \times S^{1} \rightarrow(0,1) \times S^{1}$ such that $j_{1}:(0,1)^{2} \rightarrow(0,1) \times S^{1}$ is rectilinear and $j_{2}:(0,1) \times$ $S^{1} \rightarrow(0,1) \times S^{1}$ is a shrinking embedding, cf. Definition 4.1. If $h: \mathbf{K S} \rightarrow \operatorname{Mod}_{R}^{\otimes}$ denotes a map of $\infty$-operads that encodes $\left(\mathcal{H} \mathcal{H}^{\bullet}(\mathcal{C}), \mathcal{H} \mathcal{H} \bullet(\mathcal{C})\right)$, passing to $\operatorname{Mod}_{R}$ via a coCartesian natural transformation, the image of $f_{j}$ induces a morphism in $\operatorname{Mod}_{R}$ :

$$
u: \quad \mathcal{H H}^{\bullet}(\mathcal{C}) \otimes \mathcal{H} \mathcal{H}_{\bullet}(\mathcal{C}) \simeq h(D) \otimes h\left(C_{M}\right) \rightarrow h\left(C_{M}\right) \simeq \mathcal{H} \mathcal{H}_{\bullet}(\mathcal{C})
$$


Theorem 8.2. Let $A$ be an associative algebra in $\operatorname{Mod}_{R}$ and let $\mathcal{C}$ be $\operatorname{RPerf}_{A}$. Then $u: \mathcal{H} \mathcal{H}^{\bullet}(\mathcal{C}) \otimes$ $\mathcal{H H}_{\bullet}(\mathcal{C}) \rightarrow \mathcal{H H}_{\bullet}(\mathcal{C})$ is equivalent to the contraction morphism $\sigma: \mathcal{H H}^{\bullet}(A) \otimes \mathcal{H} \mathcal{H}_{\bullet}(A) \rightarrow \mathcal{H}_{\bullet}(A)$ as a morphism in $\operatorname{Mod}_{R}$.

Example 8.3. If $\mathcal{C}=\operatorname{RPerf}_{A}$ has a Calabi-Yau structure of dimension $d$, then there is a morphism $w: \Sigma^{d} R \rightarrow \mathcal{H}_{\bullet}(A)$ in $\operatorname{Mod}_{R}$ (which we can think of as an analogue of a global section of a volume form) such that the composite $\Sigma^{d} \mathcal{H} \mathcal{H}^{\bullet}(A) \simeq \Sigma^{d} R \otimes \mathcal{H} \mathcal{H} \bullet(A) \stackrel{w \otimes \text { id }}{\longrightarrow} \mathcal{H H}^{\bullet}(A) \otimes$ $\mathcal{H H}_{\bullet}(A) \stackrel{\sigma}{\rightarrow} \mathcal{H H}_{\bullet}(A)$ is an equivalence. Here $\Sigma$ indicates the suspension. It follows from Theorem 8.2 that $\Sigma^{d} \mathcal{H}^{\bullet}(A) \rightarrow \mathcal{H}_{\bullet}(A)$ induced by $w$ and $u$ instead of $\sigma$ also gives an equivalence.

Construction 8.4. We set $\mathcal{C}=\operatorname{RPerf}_{A}$. Consider a morphism

$$
e: \operatorname{Mod}_{R} \rightarrow \operatorname{Mor}_{R}\left(\operatorname{RMod}_{A}, \operatorname{RMod}_{A}\right)
$$

in $\operatorname{Pr}_{R}^{\mathrm{L}}$ which carries $R$ to the identity functor, cf. Lemma 5.1. Applying the adjunction

$$
\text { I: } \quad \operatorname{Alg}_{\mathrm{As}_{\mathrm{s}}}\left(\operatorname{Mod}_{R}\right) \rightleftarrows\left(\operatorname{Pr}_{R}^{\mathrm{L}}\right)_{\operatorname{Mod}_{R} /}: E
$$

(see Section 5) to the morphism $\operatorname{Mod}_{R} \rightarrow \mathcal{M o r}_{R}\left(\operatorname{RMod}_{A}, \operatorname{RMod}_{A}\right)$, we have the morphisms in $\left(\operatorname{Pr}_{R}^{\mathrm{L}}\right)_{\operatorname{Mod}_{R} /}$

$$
\begin{aligned}
\operatorname{RMod}_{\mathcal{H} \mathcal{H}^{\bullet}(A) \otimes A} & \simeq \operatorname{RMod}_{\mathcal{H} \mathcal{H}^{\bullet}(A)} \otimes_{R} \operatorname{RMod}_{A} \\
& \rightarrow \operatorname{Mor}_{R}\left(\operatorname{RMod}_{A}, \operatorname{RMod}_{A}\right) \otimes_{R} \operatorname{RMod}_{A} \rightarrow \operatorname{RMod}_{A},
\end{aligned}
$$

where the right arrow is the canonical morphism, and the middle arrow is induced by the counit $\operatorname{map} \operatorname{RMod}_{\mathcal{H} \mathcal{H}^{\bullet}\left(\operatorname{RMod}_{A}\right)} \rightarrow \mathcal{M o r}_{R}\left(\operatorname{RMod}_{A}, \operatorname{RMod}_{A}\right)$ of the adjunction. Here, $\operatorname{RMod}_{\mathcal{H} \mathcal{H}^{\bullet}(A)}$ is endowed with $p_{\mathcal{H} \mathcal{H}^{\bullet}(A)}: \operatorname{Mod}_{R} \rightarrow \operatorname{RMod}_{\mathcal{H} \mathcal{H}^{\bullet}(A)}$ which carries $R$ to $\mathcal{H H}^{\bullet}(A)$. The morphisms from $\operatorname{Mod}_{R}$ are omitted from the notation. Recall that $I: \operatorname{Alg}_{\mathrm{As}_{\mathrm{s}}}\left(\operatorname{Mod}_{R}\right) \rightarrow\left(\operatorname{Pr}_{R}^{\mathrm{L}}\right)_{\operatorname{Mod}_{R} /}$ that sends $A$ to $p_{A}: \operatorname{Mod}_{R} \rightarrow \operatorname{RMod}_{A}$ with $p_{A}(R)=A$ is fully faithful so that the full subcategory of $\left(\operatorname{Pr}_{R}^{\mathrm{L}}\right)_{\operatorname{Mod}_{R} /}$ spanned by objects of the form $p_{A}: \operatorname{Mod}_{R} \rightarrow \operatorname{RMod}_{A}$ is equivalent to $\operatorname{Alg}_{\text {As }}\left(\operatorname{Mod}_{R}\right)$. Thus, the composite $\operatorname{RMod}_{\mathcal{H} \mathcal{H}^{\bullet}(A) \otimes A} \rightarrow \operatorname{RMod}_{A}$ in $\left(\operatorname{Pr}_{R}^{\mathrm{L}}\right)_{\operatorname{Mod}_{R} /}$ gives rise to a morphism of associative algebras

$$
\alpha: \mathcal{H H}^{\bullet}(A) \otimes A \rightarrow A,
$$

that is, a morphism in $\left.\operatorname{Alg}_{\text {As }}\left(\operatorname{Mod}_{R}\right)\right)$. Since

$$
\operatorname{RMod}_{A} \simeq \operatorname{Mod}_{R} \otimes_{R} \operatorname{RMod}_{A} \stackrel{e \otimes \mathrm{id}}{\rightarrow} \operatorname{Mor}_{R}\left(\operatorname{RMod}_{A}, \operatorname{RMod}_{A}\right) \otimes_{R} \operatorname{RMod}_{A} \rightarrow \operatorname{RMod}_{A}
$$

is naturally equivalent to the identity functor, we have a homotopy from the composite $A \rightarrow$ $\mathcal{H H}^{\bullet}(A) \otimes A \stackrel{\alpha}{\rightarrow} A$ to the identity morphism of $A$, where $A \rightarrow \mathcal{H H}^{\bullet}(A) \otimes A$ is induced by the morphism from the unit algebra $R \rightarrow \mathcal{H H}^{\bullet}(A)$.

We can make the following observation:

Lemma 8.5. $\mathcal{H H}^{\bullet}(A)$ is a center of $A$. See [25, Section 5.3.1] or the proof below for centers and centralizers.

Proof. The statement of this lemma is slightly imprecise. Given $B \in \mathrm{Alg}_{\mathrm{As}}\left(\operatorname{Mod}_{R}\right)$, we define $c(B, A)$ to be the fiber product $\operatorname{Map}(B \otimes A, A) \times_{\operatorname{Map}(A, A)}\left\{\operatorname{id}_{A}\right\}$, where $\operatorname{Map}(-,-)$ means the mapping space in $\operatorname{Alg}_{\mathrm{As}}\left(\operatorname{Mod}_{R}\right)$, and $\operatorname{Map}(B \otimes A, A) \rightarrow \operatorname{Map}(A, A)$ is induced by the composition with $R \otimes_{R} A \rightarrow B \otimes_{R} A$. The assignment $B \mapsto c(B, A)$ induces a functor $c(-, A): \operatorname{Alg}_{\mathrm{As}}\left(\operatorname{Mod}_{R}\right) \rightarrow \mathcal{S}$. A center of $A$ is $Z \in \operatorname{Alg}_{\text {As }}\left(\operatorname{Mod}_{R}\right)$ that represents $c(-, A)$, that is, a centralizer of the identity 
morphism $A \rightarrow A$ (cf. [25]). Through the equivalence $\operatorname{Map}(Z, Z) \simeq c(Z, A)$, the identity of $Z$ determines $Z \otimes A \rightarrow A$ and a homotopy/equivalence from the composite $A \rightarrow Z \otimes A \rightarrow A$ to id . $_{\text {. }}$ Our lemma claims that $\mathcal{H H}^{\bullet}(A)$ together with $\alpha: \mathcal{H H}^{\bullet}(A) \otimes A \rightarrow A$ and the homotopy induces an equivalence in $\mathcal{S}$ :

$$
\theta_{B}: \operatorname{Map}\left(B, \mathcal{H}^{\bullet}(A)\right) \rightarrow \operatorname{Map}(B \otimes A, A) \times_{\operatorname{Map}(A, A)}\left\{\operatorname{id}_{A}\right\}
$$

for any $B \in \operatorname{Alg}_{\text {As }}\left(\operatorname{Mod}_{R}\right)$. Here, $\operatorname{Map}\left(B, \mathcal{H} \mathcal{H}^{\bullet}(A)\right) \rightarrow \operatorname{Map}(B \otimes A, A)$ is the functor which sends $f: B \rightarrow \mathcal{H} \mathcal{H}^{\bullet}(A)$ to the composite $B \otimes A \stackrel{f \otimes \operatorname{id}_{A}}{\rightarrow} \mathcal{H} \mathcal{H}^{\bullet}(A) \otimes A \stackrel{\alpha}{\rightarrow} A$.

We will prove our claim. Namely, we show that $\theta_{B}$ is an equivalence. For ease of notation, we set $p_{B}: \operatorname{Mod}_{R} \rightarrow \operatorname{RMod}_{B}=\mathcal{M}_{B}$ and $p_{A}: \operatorname{Mod}_{R} \rightarrow \operatorname{RMod}_{A}=\mathcal{M}_{A}$. Let $X=$ $\operatorname{Map}_{\operatorname{Pr}_{R}^{\mathrm{L}}}\left(\mathcal{M}_{B} \otimes_{R} \mathcal{M}_{A}, \mathcal{M}_{A}\right) \times_{\mathcal{M} \widetilde{\widetilde{A}}}\{A\}$, where $A$ is the right $A$-module determined by the multiplication of $A$, and $\operatorname{Map}_{\operatorname{Pr}_{R}^{\mathrm{L}}}\left(\mathcal{M}_{B} \otimes_{R} \mathcal{M}_{A}, \mathcal{M}_{A}\right) \rightarrow \operatorname{Map}_{\operatorname{Pr}_{R}^{\mathrm{L}}}\left(\operatorname{Mod}_{R}, \mathcal{M}_{A}\right) \simeq \mathcal{M} \widetilde{\widetilde{A}}$ is induced by the composition with $\operatorname{Mod}_{R}=\operatorname{Mod}_{R} \otimes_{R} \operatorname{Mod}_{R} \stackrel{p_{B}}{\rightarrow} p_{A} \mathcal{M}_{B} \otimes_{R} \mathcal{M}_{A}$. Similarly, we define $Y=\operatorname{Map}_{\operatorname{Pr}_{R}^{\mathrm{L}}}\left(\mathcal{M}_{A}, \mathcal{M}_{A}\right) \times_{\mathcal{M}} \widetilde{\bar{A}}\{A\}$. Note that

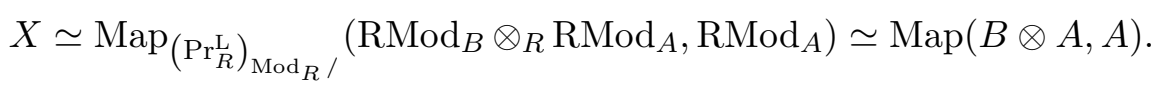

Similarly, $Y \simeq \operatorname{Map}\left(\operatorname{Pr}_{R}^{\mathrm{L}}\right)_{\operatorname{Mod}_{R} /}\left(\operatorname{RMod}_{A}, \operatorname{RMod}_{A}\right) \simeq \operatorname{Map}(A, A)$. The morphism $\operatorname{Map}_{\operatorname{Pr}_{R}^{\mathrm{L}}}\left(\mathcal{M}_{B} \otimes_{R}\right.$ $\left.\mathcal{M}_{A}, \mathcal{M}_{A}\right) \rightarrow \operatorname{Map}_{\operatorname{Pr}_{R}^{\mathrm{L}}}\left(\mathcal{M}_{A}, \mathcal{M}_{A}\right)$ given by the composition with $\operatorname{Mod}_{R} \rightarrow \mathcal{M}_{B}$ determines $X \rightarrow Y$. Let $\Delta^{0} \rightarrow Y$ be the map determined by the identity functor $\mathcal{M}_{A}$ and the identity morphism of $A$. We have a canonical equivalence

$$
X \times_{Y} \Delta^{0} \simeq \operatorname{Map}(B \otimes A, A) \times_{\operatorname{Map}(A, A)}\left\{\operatorname{id}_{A}\right\}=c(B, A) .
$$

By the universal property of $\operatorname{Mor}_{R}\left(\mathcal{M}_{A}, \mathcal{M}_{A}\right)$,

$$
\operatorname{Map}_{\operatorname{Pr}_{R}^{\mathrm{L}}}\left(\mathcal{M}_{B}, \mathcal{M o r}_{R}\left(\mathcal{M}_{A}, \mathcal{M}_{A}\right)\right) \simeq \operatorname{Map}_{\operatorname{Pr}_{R}^{\mathrm{L}}}\left(\mathcal{M}_{B} \otimes_{R} \mathcal{M}_{A}, \mathcal{M}_{A}\right)
$$

Using this equivalence, we deduce that

$$
X \times_{Y} \Delta^{0} \simeq \operatorname{Map}_{\operatorname{Pr}_{R}^{\mathrm{L}}}\left(\mathcal{M}_{B}, \mathcal{M o r}_{R}\left(\mathcal{M}_{A}, \mathcal{M}_{A}\right)\right) \times_{\mathcal{M o r}_{R}\left(\mathcal{M}_{A}, \mathcal{M}_{A}\right)} \simeq\left\{\operatorname{id}_{\mathcal{M}_{A}}\right\} .
$$

Moreover, taking into account the universal property of $\operatorname{RMod}_{\mathcal{H} \mathcal{H}^{\bullet}(A)} \rightarrow \mathcal{M o r}_{R}\left(\mathcal{M}_{A}, \mathcal{M}_{A}\right)$ in $\left(\operatorname{Pr}_{R}^{\mathrm{L}}\right)_{\operatorname{Mod}_{R} /}\left(\right.$ we omit $p_{\mathcal{H} \mathcal{H}^{\bullet}(A)}: \operatorname{Mod}_{R} \rightarrow \operatorname{RMod}_{\mathcal{H} \mathcal{H}^{\bullet}(A)}$ and $e: \operatorname{Mod}_{R} \rightarrow \mathcal{M o r}_{R}\left(\mathcal{M}_{A}, \mathcal{M}_{A}\right)$ from the notation), we see that the composition gives rise to an equivalence

$$
\begin{aligned}
& \operatorname{Map}_{\operatorname{Pr}_{R}^{\mathrm{L}}}\left(\mathcal{M}_{B}, \operatorname{RMod}_{\mathcal{H} \mathcal{H}^{\bullet}(A)}\right) \times_{\operatorname{RMod} \widetilde{\mathcal{H}} \mathcal{H}^{\bullet}(A)}\left\{\mathcal{H} \mathcal{H}^{\bullet}(A)\right\}
\end{aligned}
$$

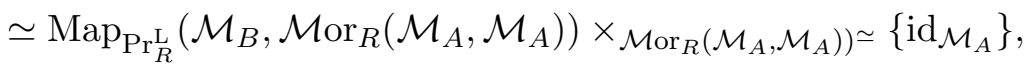

where the left hand side is naturally equivalent to $\operatorname{Map}\left(B, \mathcal{H} \mathcal{H}^{\bullet}(A)\right)$. Unwinding the construction, we have the desired equivalence $\theta_{B}: \operatorname{Map}\left(B, \mathcal{H} \mathcal{H}^{\bullet}(A)\right) \simeq c(B, A)$.

Corollary 8.6. Let us regard $\mathcal{H H}^{\bullet}(A) \otimes A$ as a right $A^{e}$-module induced by that of $A$ (that is, $\mathcal{H H}^{\bullet}(A) \otimes(-)$ means the tensor product with $\left.\mathcal{H H}^{\bullet}(A) \in \operatorname{Mod}_{R}\right)$ and regard $\alpha: \mathcal{H H}^{\bullet}(A) \otimes A \rightarrow A$ as a morphism of right $A^{e}$-moudles in the natural way. Then the morphism $\alpha: \mathcal{H H}^{\bullet}(A) \otimes A \rightarrow A$ exhibits $\mathcal{H H}^{\bullet}(A)$ as a morphism object from $A$ to itself in $\operatorname{RMod}_{A^{e}}$.

Proof. By Lemma 8.5, $\mathcal{H H}^{\bullet}(A)$ (endowed with $\alpha$ and an identification $\sigma$ between $A \rightarrow \mathcal{H}^{\bullet}(A)$ $\otimes A \rightarrow A$ and the identity morphism) is a center of $A$. According to [25, Theorem 5.3.1.30], the morphism $\mathcal{H H}^{\bullet}(A) \otimes A \rightarrow A$ of the right $A^{e}$ modules, that is obtained from the center, exhibits $\mathcal{H H}^{\bullet}(A)$ as a morphism object from $A$ to $A$. Thus, our claim follows. 
We describe the bar construction $P \otimes_{A^{e}} A$ by means of symmetric spectra. Let $\mathbb{R}$ be a cofibrant commutative symmetric ring spectrum. Let $\mathbb{A}$ be a cofibrant associative symmetric ring $\mathbb{R}$-module spectrum which represents $A$, cf. Example 6.4. We write $\wedge$ for the wedge/tensor product $\wedge_{\mathbb{R}}$ over $\mathbb{R}$. Let $B \bullet(\mathbb{A}, \mathbb{A}, \mathbb{A})$ be a simplicial diagram $\Delta^{\text {op }} \rightarrow \operatorname{Sp}^{\Sigma}(\mathbb{R})^{c}$ of symmetric spectra (called the bar construction), which is given by $[p] \mapsto \mathbb{A} \wedge \cdots \wedge \mathbb{A}=\mathbb{A} \wedge \mathbb{A}^{\wedge p} \wedge \mathbb{A}$. We refer to $\left[31\right.$, Definition 4.1.8] for the explicit formula of $B \bullet(\mathbb{A}, \mathbb{A}, \mathbb{A})$. The degeneracy maps $\mathbb{A}^{\wedge p+2} \rightarrow$ $\mathbb{A}^{\wedge p+1}$ is induced by the multiplication of $\mathbb{A} \wedge \mathbb{A} \rightarrow \mathbb{A}$, and face maps $\mathbb{A}^{\wedge p+2} \rightarrow \mathbb{A}^{\wedge p+3}$ is induced by the unit map $\mathbb{R} \rightarrow \mathbb{A}$. Each term $\mathbb{A} \wedge \mathbb{A}^{\wedge p} \wedge \mathbb{A}$ is a free left $\mathbb{A}^{e}:=\mathbb{A}^{\text {op }} \wedge \mathbb{A}$-module generated by $\mathbb{A}^{\wedge p}=\mathbb{R} \wedge \mathbb{A}^{\wedge p} \wedge \mathbb{R}$. In addition, $B \bullet(\mathbb{A}, \mathbb{A}, \mathbb{A})$ can be thought of as a simplicial diagram of left $A^{e}$-modules. The homotopy colimit of $B \cdot(\mathbb{A}, \mathbb{A}, \mathbb{A})$ is naturally equivalent to $\mathbb{A}$ with respect to stable equivalences [31, Lemma 4.1.9] so that the colimit of the induced diagram in $\operatorname{LMod}_{A^{e}}$ is $A$. Let $\mathbb{P}$ be a right $\mathbb{A}^{e}$-module which is cofibrant as an $\mathbb{R}$-module. Let $\mathbb{P} \wedge_{\mathbb{A}^{e}} B \bullet(\mathbb{A}, \mathbb{A}, \mathbb{A})$ be a simplicial diagram induced by $B_{\bullet}(\mathbb{A}, \mathbb{A}, \mathbb{A})$ which carries $[p]$ to $\mathbb{P} \wedge \mathbb{A}^{e}\left(\mathbb{A} \wedge \mathbb{A}^{\wedge p} \wedge \mathbb{A}\right) \simeq \mathbb{P} \wedge \mathbb{A}^{\wedge p}$. Consider the composition $\operatorname{Alg}_{\mathrm{As}}\left(\operatorname{Sp}^{\Sigma}(\mathbb{R})^{c}\right) \stackrel{B(-)}{\longrightarrow} \operatorname{Cat}_{\mathbb{R}}^{\mathrm{pc}} \stackrel{\mathcal{H} \mathcal{H}(-)}{\longrightarrow} \operatorname{Fun}\left(\Lambda^{\mathrm{op}}, \operatorname{Sp}^{\Sigma}(\mathbb{R})^{c}\right) \rightarrow \operatorname{Fun}\left(\Delta^{\mathrm{op}}, \mathrm{Sp}^{\Sigma}(\mathbb{R})^{c}\right)$, where the final morphism is determined by the restriction $\Delta^{\mathrm{op}} \subset \Lambda^{\mathrm{op}}$. We write $\mathcal{H}^{\Delta}(-)$ for the composite. Note that $\mathcal{H} \mathcal{H}_{\bullet}^{\Delta}(\mathbb{A})$ gives rise to a simplicial diagram in $\operatorname{Mod}_{R}$ whose colimit is $\mathcal{H}_{\bullet}(A)$. The standard computation shows that $\mathbb{A} \wedge_{\mathbb{A}}^{e} B_{\bullet}(\mathbb{A}, \mathbb{A}, \mathbb{A})$ can be identified with $\mathcal{H}_{\bullet}^{\Delta}(\mathbb{A})$.

Lemma 8.7. Let $\mathbb{P}$ be a right $\mathbb{A}^{e}$-module symmetric spectrum which is cofibrant as an $\mathbb{R}$-module. We write $P$ for the image of $\mathbb{P}$ in $\operatorname{RMod}_{A^{e}}$. Then $P \otimes_{A^{e}} A$ can be identified with a colimit of the simplicial diagram induced by $\mathbb{P} \wedge_{\mathbb{A}^{e}} B_{\bullet}(\mathbb{A}, \mathbb{A}, \mathbb{A})$. In particular, $\mathcal{H} \mathcal{H}_{\bullet}(A)$ can be identified with $A \otimes_{A^{e}} A$ in $\operatorname{Mod}_{R}$.

Proof. Note that the two-sided bar construction preserves colimits in each variable. Moreover, the colimit of $B \bullet(\mathbb{A}, \mathbb{A}, \mathbb{A})$ is $A$ after passing to $\operatorname{LMod}_{A^{e}}$, and each $\mathbb{P} \wedge \mathbb{A}^{e}\left(\mathbb{A} \wedge \mathbb{A}^{\wedge p} \wedge \mathbb{A}\right) \simeq \mathbb{P} \wedge \mathbb{A}^{\wedge p}$ computes $P \otimes A^{\otimes p} \simeq P \otimes_{A^{e}}\left(A \otimes A^{\otimes p} \otimes A\right)$. Therefore, lemma follows.

Proof of Theorem 8.2. We use the notation in the discussion about the setting of Theorem 8.2. We first consider an operadic left Kan extension $h^{\prime}: \overline{\mathbf{D C y l}} \rightarrow \operatorname{Mod}_{R}^{\otimes}$ of $h: \mathbf{K S} \rightarrow \operatorname{Mod}_{R}^{\otimes}$ over $\Gamma$ along $\mathbf{K S} \hookrightarrow \overline{\mathbf{D C y l}}$, cf. Proposition 4.20. The open embedding $j:(0,1)^{2} \sqcup(0,1) \times S^{1} \stackrel{j_{1} \sqcup j_{2}}{\longrightarrow}$ $(0,1) \times S^{1}$ factors as the composition of two open embeddings $(0,1)^{2} \sqcup(0,1) \times S^{1} \stackrel{d \sqcup \text { id }}{\longrightarrow}(0,1) \times S^{1} \sqcup$ $(0,1) \times S^{1} \stackrel{r \sqcup j_{2}}{\rightarrow}(0,1) \times S^{1}$. See Definition 4.3 for the notation and convention. The embedding $d:(0,1)^{2} \rightarrow(0,1) \times S^{1}$ and $r:(0,1) \times S^{1} \rightarrow(0,1) \times S^{1}$ are recti-linear embeddings such that the composite $r \circ d$ is $j_{1}$ (of course, the image of $r$ does not intersect with that of the shrinking embedding $\left.j_{2}\right)$. Thus, $f_{j}$ factors as $\left(\langle 2\rangle, D, C_{M}\right) \rightarrow\left(\langle 2\rangle, C, C_{M}\right) \rightarrow\left(\langle 1\rangle, C_{M}\right)$ in $\overline{\mathbf{D C y}}$, that lie over $\langle 2\rangle=\langle 2\rangle \stackrel{\rho}{\rightarrow}\langle 1\rangle$. It follows that $h(D) \otimes h\left(C_{M}\right) \rightarrow h\left(C_{M}\right)$ induced by $f_{j}$ factors as

$$
h(D) \otimes h\left(C_{M}\right) \simeq h^{\prime}(D) \otimes h^{\prime}\left(C_{M}\right) \rightarrow h^{\prime}(C) \otimes h^{\prime}\left(C_{M}\right) \rightarrow h^{\prime}\left(C_{M}\right) \simeq h\left(C_{M}\right)
$$

where $h^{\prime}(D) \rightarrow h^{\prime}(C)$ and $h^{\prime}(C) \otimes h^{\prime}\left(C_{M}\right) \rightarrow h^{\prime}\left(C_{M}\right)$ are induced by $d$ and $r \sqcup j_{2}$, respectively.

Next, we describe $h^{\prime}(D) \rightarrow h^{\prime}(C)$ in an explicit way. If $\mathcal{H H}^{\bullet}(A)$ is encoded by the map of an $\infty$-operads $\mathbf{E}_{2}^{\otimes} \rightarrow \operatorname{Mod}_{R}^{\otimes}$, we let $i_{!}\left(\mathcal{H H}^{\bullet}(A)\right): \mathbf{D C y l} \rightarrow \operatorname{Mod}_{R}^{\otimes}$ be its operadic left Kan extension along $i$ : $\mathbf{E}_{2}^{\otimes} \rightarrow$ DCyl. Let $i_{!}\left(\mathcal{H H}^{\bullet}(A)\right)((\langle 1\rangle, D)) \rightarrow i_{!}\left(\mathcal{H} \mathcal{H}^{\bullet}(A)\right)((\langle 1\rangle, C))$ be a morphism in $\operatorname{Mod}_{R}$, that is determined by $d$. For simplicity, we set $Z:=\mathcal{H H}^{\bullet}(A)$ and we write $i_{!}(Z)(D) \rightarrow i_{!}(Z)(C)$ for this morphism. If we think of $Z$ as the underlying $\mathbf{E}_{1}$-algebra given by the composite $\mathbf{E}_{1}^{\otimes} \rightarrow \mathbf{E}_{2}^{\otimes} \rightarrow \operatorname{Mod}_{R}^{\otimes}$, we denote by $l_{!}(Z): \mathbf{M f l d}_{1} \rightarrow \operatorname{Mod}_{R}^{\otimes}$ its operadic left Kan extension along $l: \mathbf{E}_{1}^{\otimes} \hookrightarrow \mathbf{M f l d}_{1}$. A rectilinear embedding $(0,1) \rightarrow S^{1}$ gives rise to $l_{!}(Z)((0,1)) \rightarrow l_{!}(Z)\left(S^{1}\right)$ in $\operatorname{Mod}_{R}$ (we abuse notation as above). More explicitly, $l_{!}(Z)((0,1)) \rightarrow l_{!}(Z)\left(S^{1}\right)$ is $Z=\tilde{Z}((0,1)) \rightarrow \operatorname{colim}_{U \rightarrow S^{1} \in\left(\text { Disk }_{1}\right)_{/ S}} \tilde{Z}(U)$, where $Z$ means the 
underlying $R$-module, and $\tilde{Z}:$ Disk $_{1} \rightarrow \operatorname{Mod}_{R}$ is the underlying functor of the symmetric monoidal functor $\tilde{Z}$ : Disk ${ }_{1}^{\otimes} \rightarrow \operatorname{Mod}_{R}^{\otimes}$ corresponding to the composite $\mathbf{E}_{1}^{\otimes} \rightarrow \mathbf{E}_{2}^{\otimes} \rightarrow \operatorname{Mod}_{R}^{\otimes}$. Since the forgetful functor $\operatorname{Alg}_{\mathbf{E}_{1}}\left(\operatorname{Mod}_{R}\right) \rightarrow \operatorname{Mod}_{R}$ preserves colimits over the sifted category $\left(\text { Disk }_{1}\right)_{/ S^{1}}$, it follows from Lemma 7.13 that $i_{!}(Z)(D) \rightarrow i_{!}(Z)(C)$ is naturally equivalent to $Z=l_{!}(Z)((0,1)) \rightarrow l_{!}(Z)\left(S^{1}\right)=\operatorname{colim}_{\left(\text {Disk }_{1}\right)_{/ S 1}} \tilde{Z}(U)$ as a morphism in $\operatorname{Mod}_{R}$. Therefore, we may identify $h^{\prime}(D) \rightarrow h^{\prime}(C)$ with $Z=l_{!}(Z)((0,1)) \rightarrow l_{!}(Z)\left(S^{1}\right)=\operatorname{colim}_{\left(\text {Disk }_{1}\right)_{/ S^{1}}} \tilde{Z}(U)$. Henceforth, we regard $Z$ as an associative algebra in $\operatorname{Mod}_{R}$, which is given by a symmetric monoidal functor $\widetilde{\mathrm{As}}^{\otimes} \rightarrow \operatorname{Mod}_{R}^{\otimes}$. The map $\xi: \Lambda^{\mathrm{op}} \rightarrow \widetilde{\mathrm{As}}^{\otimes}$ (see the discussion proceeding to Lemma 7.12) and $\Delta^{\mathrm{op}} \hookrightarrow \Lambda_{\infty}^{\mathrm{op}} \rightarrow \Lambda^{\mathrm{op}}$ induces

$$
\begin{aligned}
\operatorname{Alg}_{\mathrm{As}}\left(\operatorname{Mod}_{R}\right) & \simeq \operatorname{Fun}^{\otimes}\left(\widetilde{\mathrm{As}}^{\otimes}, \operatorname{Mod}_{R}^{\otimes}\right) \rightarrow \operatorname{Fun}\left(\Lambda^{\mathrm{op}}, \operatorname{Mod}_{R}\right) \\
& \rightarrow \operatorname{Fun}\left(\Lambda_{\infty}^{\mathrm{op}}, \operatorname{Mod}_{R}\right) \rightarrow \operatorname{Fun}\left(\Delta^{\mathrm{op}}, \operatorname{Mod}_{R}\right) .
\end{aligned}
$$

It follows from Lemma 7.6 that the image of $Z$ in $\operatorname{Fun}\left(\Lambda_{\infty}^{\mathrm{op}}, \operatorname{Mod}_{R}\right)$ is the composite $\Lambda_{\infty}^{\mathrm{op}} \simeq$ $\left(\text { Disk }_{1}^{\dagger}\right)_{/ S^{1}} \rightarrow$ Disk $_{1} \rightarrow \operatorname{Mod}_{R}$ whose colimit is naturally equivalent to $l_{!}(Z)\left(S^{1}\right)$. Moreover, from the cofinality of $\Delta^{\mathrm{op}} \rightarrow \Lambda_{\infty}^{\mathrm{op}}, l_{!}(Z)\left(S^{1}\right)$ is naturally equivalent to the colimit of $c: \Delta^{\mathrm{op}} \rightarrow \Lambda_{\infty}^{\mathrm{op}} \rightarrow$ $\operatorname{Mod}_{R}$. Taking into account Remark 7.7, $Z \rightarrow l_{!}(Z)\left(S^{1}\right)$ can be identified with $Z=c([0]) \rightarrow$ $\operatorname{colim}_{[p] \in \Delta^{\mathrm{op}}} c([p])$.

Next let us consider $h^{\prime}(C) \otimes h^{\prime}\left(C_{M}\right) \rightarrow h^{\prime}\left(C_{M}\right)$. According to Construction 7.18, its underlying morphism in $\operatorname{Mod}_{R}$ can naturally be identified with $\mathcal{H H}_{\bullet}(Z) \otimes \mathcal{H} \mathcal{H}_{\bullet}(A) \simeq \mathcal{H}_{\bullet}(Z \otimes A) \rightarrow$ $\mathcal{H} \mathcal{H}_{\bullet}(A)$ induced by $\alpha: Z \otimes A \rightarrow A$. Let $\mathbb{Z}$ and $\mathbb{A}$ be cofibrant and fibrant associative ring symmetric $\mathbb{R}$-module spectra that represent $Z$ and $A$, respectively (namely, they are objects in $\operatorname{Alg}_{\mathrm{As}}\left(\mathrm{Sp}^{\Sigma}(\mathbb{R})\right)$ which are both cofibrant and fibrant with respect to the projective model structure). Let $\bar{\alpha}: \mathbb{Z} \wedge \mathbb{A} \rightarrow \mathbb{A}$ be a morphism in $\operatorname{Alg}_{\mathrm{As}}\left(\operatorname{Sp}^{\Sigma}(\mathbb{R})^{c}\right)$ which represents $\alpha$. The composite $\mathbb{A} \simeq \mathbb{R} \wedge \mathbb{A} \rightarrow \mathbb{Z} \wedge \mathbb{A} \rightarrow \mathbb{A}$ induced by $\mathbb{R} \rightarrow \mathbb{Z}$ is equivalent to the identity morphism of $\mathbb{A}$. Let $\mathcal{H H}_{\bullet}^{\Delta}(\mathbb{Z}) \wedge \mathcal{H} \mathcal{H}_{\bullet}^{\Delta}(\mathbb{A})$ denote the bisimplicial diagram induced by the wedge product and $\left(\mathcal{H H}_{\bullet}^{\Delta}(\mathbb{Z}) \wedge \mathcal{H H}_{\bullet}^{\Delta}(\mathbb{A})\right)^{\text {diag }}$ the associated diagonal simplicial diagram. The morphism $\bar{\alpha}$ induces the following morphism of simplicial diagrams

$$
\left(\mathcal{H}_{\bullet}^{\Delta}(\mathbb{Z}) \wedge \mathcal{H} \mathcal{H}_{\bullet}^{\Delta}(\mathbb{A})\right)^{\operatorname{diag}} \simeq \mathcal{H} \mathcal{H}_{\bullet}^{\Delta}(\mathbb{Z} \wedge \mathbb{A}) \rightarrow \mathcal{H} \mathcal{H}_{\bullet}^{\Delta}(\mathbb{A})
$$

whose colimit is equivalent to $\mathcal{H}_{\bullet}(Z) \otimes \mathcal{H}_{\bullet}(A) \simeq \mathcal{H}_{\bullet}(Z \otimes A) \rightarrow \mathcal{H}_{\bullet}(A)$ (notice that $\Delta^{\text {op }}$ is sifted).

Note that $l_{!}(Z)\left(S^{1}\right)=\operatorname{colim}_{[p] \in \Delta^{\text {op }}} c([p]) \simeq \mathcal{H} \mathcal{H} \bullet(Z)$. If $\mathbb{Z}_{c s t}$ denotes the constant simplicial diagram taking the value $\mathbb{Z},\{[0]\} \rightarrow \Delta^{\text {op }}$ induces $\mathbb{Z}_{c s t} \rightarrow \mathcal{H H}_{\bullet}^{\Delta}(\mathbb{Z})$ whose colimit is $Z=$ $c([0]) \rightarrow \operatorname{colim}_{[p] \in \Delta^{\mathrm{op}}} c([p]) \simeq \mathcal{H H}_{\bullet}(Z)$. Consider $\mathbb{Z}_{c s t} \wedge \mathcal{H} \mathcal{H}_{\bullet}^{\Delta}(\mathbb{A}) \rightarrow\left(\mathcal{H H}_{\bullet}^{\Delta}(\mathbb{Z}) \wedge \mathcal{H} \mathcal{H}_{\bullet}^{\Delta}(\mathbb{A})\right)^{\operatorname{diag}} \rightarrow$ $\mathcal{H H}_{\bullet}^{\Delta}(\mathbb{A})$. Their colimits give $Z \otimes \mathcal{H} \mathcal{H}_{\bullet}(A) \rightarrow \mathcal{H} \mathcal{H}_{\bullet}(Z) \otimes \mathcal{H} \mathcal{H}_{\bullet}(A) \rightarrow \mathcal{H} \mathcal{H}_{\bullet}(A)$, which is equivalent to $h^{\prime}(D) \otimes h^{\prime}\left(C_{M}\right) \rightarrow h^{\prime}(C) \otimes h^{\prime}\left(C_{M}\right) \rightarrow h^{\prime}\left(C_{M}\right)$. Observe that the composition $\mathbb{Z}_{c s t} \wedge \mathcal{H}_{\bullet}^{\Delta}(\mathbb{A})=(\mathbb{Z} \wedge \mathbb{A}) \wedge_{\mathbb{A} e} B_{\bullet}(\mathbb{A}, \mathbb{A}, \mathbb{A}) \rightarrow \mathbb{A} \wedge_{\mathbb{A}^{e}} B_{\bullet}(\mathbb{A}, \mathbb{A}, \mathbb{A})=\mathcal{H H}_{\bullet}^{\Delta}(\mathbb{A})$ is induced by $\bar{\alpha}: \mathbb{Z} \wedge \mathbb{A} \rightarrow \mathbb{A}$. The contraction morphism $(Z \otimes A) \otimes_{A^{e}} A \rightarrow A \otimes_{A^{e}} A$ is obtained from $\mathbb{Z} \wedge\left(\mathbb{A} \wedge_{\mathbb{A}^{e}} B_{\bullet}(\mathbb{A}, \mathbb{A}, \mathbb{A})\right)=(\mathbb{Z} \wedge \mathbb{A}) \wedge_{\mathbb{A}^{e}} B \bullet(\mathbb{A}, \mathbb{A}, \mathbb{A}) \rightarrow \mathbb{A} \wedge_{\mathbb{A}^{e}} B \bullet(\mathbb{A}, \mathbb{A}, \mathbb{A})$ by taking colimits. This completes the proof.

8.2. Let $k$ be a field. We suppose that $R$ is the Eilenberg-MacLane spectrum of $k$. We write $k$ for $R$. In this context, we will give a concrete model of the contraction morphism

$$
\sigma: \mathcal{H H}^{\bullet}(A) \otimes \mathcal{H H}_{\bullet}(A) \rightarrow \mathcal{H}_{\bullet}(A)
$$

as a morphism of chain complexes of $k$-vector spaces. Let $\operatorname{Comp}^{\otimes}(k)$ be the symmetric monoidal category of chain complexes of $k$-vector spaces, whose tensor product is given by the standard 
tensor product of chain complexes. There is a symmetric monoidal (projective) model structure on $\operatorname{Comp}(k)$ such that a morphism is a weak equivalence (resp. a fibration) if it is a quasiisomorphism (resp. a termwise surjective), see, e.g., [25, Proposition 7.1.2.11]. Since $k$ is a field, every object is both cofibrant and fibrant. Let $\operatorname{Comp}(k)\left[W^{-1}\right]^{\otimes}$ be the symmetric monoidal $\infty$ category obtained by inverting quasi-isomorphisms. We fix a symmetric monoidal equivalence $\operatorname{Mod}_{k}^{\otimes} \simeq \operatorname{Comp}(k)\left[W^{-1}\right]^{\otimes}$, see [25, Theorem 7.1.2.13]. Thus it gives rise to $\operatorname{Alg}_{\mathrm{As}}(\operatorname{Comp}(k)) \rightarrow$ $\operatorname{Alg}_{\mathrm{As}}\left(\operatorname{Comp}(k)\left[W^{-1}\right]\right) \simeq \operatorname{Alg}_{\mathrm{As}}\left(\operatorname{Mod}_{k}\right)$. We here regard $\operatorname{Alg}_{\mathrm{As}}(\operatorname{Comp}(k))$ as (the nerve of) the category of differential graded $k$-algebras in an obvious way. Let A be an associative (cofibrant) differential graded $k$-algebra, i.e., an object of $\operatorname{Alg}_{\mathrm{As}}(\operatorname{Comp}(k))$ that represents $A \in$ $\operatorname{Alg}_{\mathrm{As}}\left(\operatorname{Mod}_{k}\right)$. Let $\mathrm{A}^{e}:=\mathrm{A}^{\mathrm{op}} \otimes \mathrm{A}$. The natural functor $\operatorname{Comp}(k)^{\otimes} \rightarrow \operatorname{Comp}(k)\left[W^{-1}\right]^{\otimes} \simeq \operatorname{Mod}_{k}^{\otimes}$ induces

$$
\operatorname{RMod}_{\mathrm{A}^{e}}(\operatorname{Comp}(k)) \times \operatorname{LMod}_{\mathrm{A}^{e}}(\operatorname{Comp}(k)) \rightarrow \operatorname{RMod}_{A^{e}}\left(\operatorname{Mod}_{k}\right) \times \operatorname{LMod}_{A^{e}}\left(\operatorname{Mod}_{k}\right) \rightarrow \operatorname{Mod}_{k},
$$

where the right functor is informally given by the bar construction (i.e., the relative tensor product) $(-) \otimes_{A^{e}}(-)$. We describe the contraction morphism by using explicit resolutions. Let $B_{\bullet}^{\mathrm{dg}}(\mathrm{A}):=B_{\bullet}^{\mathrm{dg}}(\mathrm{A}, \mathrm{A}, \mathrm{A})$ be the right $\mathrm{A}^{e}$-module associated to the total complex of the simplicial diagram of $\mathrm{A}^{e}$-modules $[p] \mapsto \mathrm{A} \otimes \mathrm{A}^{\otimes p} \otimes \mathrm{A}$ (defined as in $B \bullet(\mathbb{A}, \mathbb{A}, \mathbb{A})$ ). The associated total complex $B_{\bullet}^{\mathrm{dg}}(\mathrm{A})$ computes a homotopy colimit of the simplicial diagram, and there is a canonical morphism of right $A^{e}$-modules $B_{\bullet}^{\mathrm{dg}}(\mathrm{A}) \rightarrow \mathrm{A}$ which is a quasi-isomorphism. Let $\operatorname{Hom}_{\mathrm{A}^{e}}\left(B_{\bullet}^{\mathrm{dg}}(\mathrm{A}), B_{\bullet}^{\mathrm{dg}}(\mathrm{A})\right)$ be the hom chain complex of right $\mathrm{A}^{e}$-modules. By Lemma 8.9 below, $\operatorname{Hom}_{\mathrm{A}^{e}}\left(B_{\bullet}^{\mathrm{dg}}(\mathrm{A}), B_{\bullet}^{\mathrm{dg}}(\mathrm{A})\right)$ represents/computes the (derived) hom complex from $\mathrm{A}$ to $\mathrm{A}$ in $\operatorname{RMod}_{A^{e}}(\operatorname{Comp}(k))$. Let us consider an evaluation morphism of right $A^{e}$-modules

$$
\mathrm{Ev}: \operatorname{Hom}_{\mathrm{A}^{e}}\left(B_{\bullet}^{\mathrm{dg}}(\mathrm{A}), B_{\bullet}^{\mathrm{dg}}(\mathrm{A})\right) \otimes B_{\bullet}^{\mathrm{dg}}(\mathrm{A}) \rightarrow B_{\bullet}^{\mathrm{dg}}(\mathrm{A})
$$

defined in the obvious way, where $\operatorname{Hom}_{\mathrm{A}^{e}}\left(B_{\bullet}^{\mathrm{dg}}(\mathrm{A}), B_{\bullet}^{\mathrm{dg}}(\mathrm{A})\right) \otimes B_{\bullet}^{\mathrm{dg}}(\mathrm{A})$ comes equipped with the right $\mathrm{A}^{e}$-module structure induced by $B_{\bullet}^{\mathrm{dg}}(\mathrm{A})$. Let $\operatorname{RMod}_{\mathrm{A}^{e}}(\operatorname{Comp}(k))\left[W^{-1}\right]$ denote the $\infty$-category obtained by inverting quasi-isomorphisms (after restricting to cofibrant objects). By the universal property of the morphism $\left(\operatorname{Hom}_{\mathrm{A}^{e}}\left(B_{\bullet}^{\mathrm{dg}}(\mathrm{A}), B_{\bullet}^{\mathrm{dg}}(\mathrm{A})\right), \mathrm{Ev}\right)$ and the equivalence

$$
\operatorname{RMod}_{\mathrm{A}^{e}}(\operatorname{Comp}(k))\left[W^{-1}\right] \simeq \operatorname{RMod}_{A^{e}}\left(\operatorname{Mod}_{k}\right)
$$

[25, Theorem 4.3.3.17] together with Lemma 8.9, it represents a morphism object from $A$ to $A$ in $\operatorname{RMod}_{A^{e}}$. Note that $B_{\bullet}^{\mathrm{dg}}(\mathrm{A}) \otimes_{\mathrm{A}^{e}} \mathrm{~A}$ is the chain complex associated to the simplicial chain complex given by $[p] \mapsto\left(\mathrm{A} \otimes \mathrm{A}^{\otimes p} \otimes \mathrm{A}\right) \otimes_{\mathrm{A}^{e}} \mathrm{~A} \simeq \mathrm{A}^{\otimes p} \otimes \mathrm{A}$ so that the associated complex is a model of $A \otimes_{A^{e}} A=\mathcal{H H}_{\bullet}(A)$. That is, its image in $\operatorname{Mod}_{k}$ is naturally equivalent to $B_{\bullet}^{\mathrm{dg}}(\mathrm{A}) \otimes_{\mathrm{A}^{e}} B_{\bullet}^{\mathrm{dg}}(\mathrm{A}) \stackrel{\sim}{\rightarrow} \mathrm{A} \otimes_{\mathrm{A}^{e}} B_{\bullet}^{\mathrm{dg}}(\mathrm{A})=\mathcal{H} \mathcal{H}_{\bullet}(A)$. By the Morita theory (cf., e.g., [5, Section 4]), $\mathcal{M o r}_{k}\left(\operatorname{RMod}_{A}, \operatorname{RMod}_{A}\right) \simeq \operatorname{RMod}_{A^{e}}$, where the identity functor amounts to $A \in \operatorname{RMod}_{A^{e}}$ having the diagonal module structure. Consequently, $\operatorname{Hom}_{\mathrm{A}^{e}}\left(B_{\bullet}^{\mathrm{dg}}(\mathrm{A}), B_{\bullet}^{\mathrm{dg}}(\mathrm{A})\right) \in$ $\operatorname{Comp}(k)$ is a model of $\mathcal{H H}^{\bullet}(A) \in \operatorname{Mod}_{k}$. Moreover, $B_{\bullet}^{\mathrm{dg}}(\mathrm{A}) \rightarrow \mathrm{A}$ induces a quasi-isomorphism $\operatorname{Hom}_{\mathrm{A}^{e}}\left(B_{\bullet}^{\mathrm{dg}}(\mathrm{A}), B_{\bullet}^{\mathrm{dg}}(\mathrm{A})\right) \rightarrow \operatorname{Hom}_{\mathrm{A}^{e}}\left(B_{\bullet}^{\mathrm{dg}}(\mathrm{A}), \mathrm{A}\right)$ (perhaps, the latter is more common Hochschild cochain complex). We conclude:

Proposition 8.8. The above evaluation morphism induces

$$
\operatorname{Hom}_{\mathrm{A}^{e}}\left(B_{\bullet}^{\mathrm{dg}}(\mathrm{A}), B_{\bullet}^{\mathrm{dg}}(\mathrm{A})\right) \otimes B_{\bullet}^{\mathrm{dg}}(\mathrm{A}) \otimes_{\mathrm{A}^{e}} \mathrm{~A} \rightarrow B_{\bullet}^{\mathrm{dg}}(\mathrm{A}) \otimes_{\mathrm{A}^{e}} \mathrm{~A},
$$

which is equivalent to the contraction morphism $\sigma: \mathcal{H H}^{\bullet}(A) \otimes \mathcal{H} \mathcal{H} \bullet(A) \rightarrow \mathcal{H} \mathcal{H}_{\bullet}(A)$. In particular, it is one of (explicit) models of $u$ (cf. Theorem 8.2). 
Lemma 8.9. Let us consider $\operatorname{RMod}_{\mathrm{A}^{e}}(\operatorname{Comp}(k))$ to be a category endowed with $\operatorname{Comp}(k)$ enriched projective model structure, where a morphism is a weak equivalence (resp. a fibration) if it is a quasi-isomorphism (resp. a termwise surjective), see, e.g., [3, Theorem 3.3]. Then $B_{\bullet}^{\mathrm{dg}}(\mathrm{A})$ is cofibrant with respect to this model structure.

Proof. Since $B_{\bullet}^{\mathrm{dg}}(\mathrm{A})$ is obtained from the simplicial chain complex $[p] \mapsto \mathrm{A} \otimes \mathrm{A}^{\otimes p} \otimes \mathrm{A}$, there is an increasing filtration of $\mathrm{A}^{e}$-submodules $0=F_{-1}\left(B_{\bullet}^{\mathrm{dg}}(\mathrm{A})\right) \hookrightarrow F_{0}\left(B_{\bullet}^{\mathrm{dg}}(\mathrm{A})\right) \hookrightarrow F_{1}\left(B_{\bullet}^{\mathrm{dg}}(\mathrm{A})\right) \hookrightarrow$ .. such that $\cup_{p \geq 0} F_{p}\left(B_{\bullet}^{\mathrm{dg}}(\mathrm{A})\right)=B_{\bullet}^{\mathrm{dg}}(\mathrm{A})$. The quotient $F_{p+1}\left(B_{\bullet}^{\mathrm{dg}}(\mathrm{A})\right) / F_{p}\left(B_{\bullet}^{\mathrm{dg}}(\mathrm{A})\right)$ is isomorphic to $\mathrm{A} \otimes \overline{\mathrm{A}}^{\otimes p+1} \otimes \mathrm{A}$ as a right $\mathrm{A}^{e}$-module, where $\overline{\mathrm{A}}$ is the cokernel of a unit morphism $k \rightarrow \mathrm{A}$. That is, $F_{p+1}\left(B_{\bullet}^{\mathrm{dg}}(\mathrm{A})\right) / F_{p}\left(B_{\bullet}^{\mathrm{dg}}(\mathrm{A})\right)$ is a free right $\mathrm{A}^{e}$-module generated by $\overline{\mathrm{A}}^{\otimes p+1} \in \mathrm{Comp}(k)$. By [3, Definition 9.17, Theorem 9.20], the existence of this filtration implies that $B_{\bullet}^{\mathrm{dg}}(\mathrm{A})$ is cofibrant with respect to the $r$-model structure in [3, Section 4]. We deduce from the assumption that $k$ is a field that the projective model structure coincides with this $r$-model structure (the projective model structure is the same as the $q$-model structure in [3, Section 3]). This completes the proof.

Remark 8.10. There are other operations between $\mathcal{H H}^{\bullet}(A) \otimes \mathcal{H} \mathcal{H} \bullet(A)$ and $\mathcal{H} \mathcal{H} \bullet(A)$, which is induced by the $\mathbf{K S}$-algebra structure on $\left(\mathcal{H H}^{\bullet}(A), \mathcal{H H}^{\bullet}(A)\right)$. We continue to work with the coefficient field $R=k$. We further assume that $k$ is of characteristic zero. Observe first that the Kan complex $\operatorname{Mult}_{\mathrm{KS}}\left(\left\{D, C_{M}\right\}, C_{M}\right)$ is equivalent to the product of the circles $S^{1} \times S^{1}$ in $\mathcal{S}$, where $S^{1} \times S^{1}$ is regarded as an object of $\mathcal{S}$. The left factor $S^{1}$ is homotopy equivalent to the space of configuration of one point on $(0,1) \times S^{1}$, which we regard as the space of rectilinear embeddings from $(0,1)^{2}$ into $(0,1) \times S^{1}$. The right factor $S^{1}$ can be identified with the mapping space from $C_{M}$ to itself, that is, the space of shrinking embeddings $(0,1) \times S^{1} \rightarrow(0,1) \times S^{1}$. The map of $\infty$ operads $h: \mathbf{K S} \rightarrow \operatorname{Mod}_{k}^{\otimes}$ encoding the $\mathbf{K S}$-algebra $\left(\mathcal{H} \mathcal{H}^{\bullet}(A), \mathcal{H} \mathcal{H} \bullet(A)\right)$ in Theorem 7.14 induces morphisms $h_{\left(\left\{D, C_{M}\right\}, C_{M}\right)}: \operatorname{Mult}_{\mathrm{KS}}\left(\left\{D, C_{M}\right\}, C_{M}\right) \rightarrow \operatorname{Map}_{\operatorname{Mod}_{k}}\left(\mathcal{H} \mathcal{H}^{\bullet}(A) \otimes \mathcal{H} \mathcal{H} \bullet(A), \mathcal{H} \mathcal{H} \bullet(A)\right)$ and $h_{\left(\left\{C_{M}\right\}, C_{M}\right)}: \operatorname{Mult}_{\mathrm{KS}}\left(\left\{C_{M}\right\}, C_{M}\right) \rightarrow \operatorname{Map}_{\operatorname{Mod}_{k}}\left(\mathcal{H} \mathcal{H}_{\bullet}(A), \mathcal{H} \mathcal{H} \bullet(A)\right)$ in $\mathcal{S}$. To discuss operations induced by these morphisms, we adopt the differential graded setting (we simplify the problem). By taking the singular chain complex of the simplicial sets $\operatorname{Mult}_{\mathrm{KS}}(-,-)$ with coefficients in $k$, we obtain the differential graded ( $\mathrm{dg}$ ) operad $C \bullet(\mathrm{KS})$ from KS, i.e., an operad in the symmetric monoidal category $\operatorname{Comp}(k)$. We refer to [15] for the relation between $\mathrm{dg}$ operads and $\infty$-operads. By a rectification result of Hinich [15, Theorem 4.1.1], there is a canonical equivalence between $\operatorname{Alg}_{\mathbf{K S}}\left(\operatorname{Mod}_{k}\right)$ and the $\infty$-category of $C_{\bullet}(\mathrm{KS})$-algebras in $\operatorname{Comp}(k)$ in the "conventional" sense so that the $\mathbf{K S}$-algebra $\left(\mathcal{H} \mathcal{H}^{\bullet}(A), \mathcal{H} \mathcal{H} \bullet(A)\right)$ gives rise to a (an essentially unique) $C$ •(KS)-algebra in $\operatorname{Comp}(k)$ in the "conventional" sense: see [15, Section 2.2.4] (we here use the assumption of characteristic zero). We denote this $C_{\bullet}(\mathrm{KS})$-algebra by $\left(H H^{\bullet}(A), H_{\bullet}(A)\right)$. Then the $C_{\bullet}(\mathrm{KS})$-algebra $\left(H H^{\bullet}(A), H H_{\bullet}(A)\right)$ induces morphisms into hom chain complexes:

$$
h_{\left(\left\{D, C_{M}\right\}, C_{M}\right)}^{\mathrm{dg}}: C_{\bullet}\left(\operatorname{Mult}_{\mathrm{KS}}\left(\left\{D, C_{M}\right\}, C_{M}\right)\right) \rightarrow \operatorname{Hom}_{k}\left(H H^{\bullet}(A) \otimes H_{\bullet}(A), H H_{\bullet}(A)\right)
$$

and

$$
h_{\left(\left\{C_{M}\right\}, C_{M}\right)}^{\mathrm{dg}}: C_{\bullet}\left(\operatorname{Mult}_{\mathrm{KS}}\left(\left\{C_{M}\right\}, C_{M}\right)\right) \rightarrow \operatorname{Hom}_{k}\left(H H_{\bullet}(A), H H_{\bullet}(A)\right)
$$

in $\operatorname{Comp}(k)\left[W^{-1}\right]$. Given a simplicial set $S$, we write $H_{*}(S)$ for the homology $H_{*}\left(C_{\bullet}(S)\right)$ of the singular chain complex $C \bullet(S)$ of $S$ with coefficients in $k$. Passing to homology, we obtain a morphism of graded $k$-vector spaces

$$
\begin{aligned}
H_{*}\left(h_{\left(\left\{D, C_{M}\right\}, C_{M}\right)}^{\mathrm{dg}}\right): & H_{*}\left(\operatorname{Mult}_{\mathrm{KS}}\left(\left\{D, C_{M}\right\}, C_{M}\right)\right) \\
& \rightarrow H_{*}\left(\operatorname{Hom}_{k}\left(H H^{\bullet}(A) \otimes H H_{\bullet}(A), H H_{\bullet}(A)\right)\right)
\end{aligned}
$$


from $h_{\left(\left\{D, C_{M}\right\}, C_{M}\right)}^{\mathrm{dg}}$. Note that by definition the left-hand side is connective. This map can also be obtained by taking homology of $C_{\bullet}\left(h_{\left(\left\{D, C_{M}\right\}, C_{M}\right)}\right)$ up to isomorphisms. Since the mapping space $\operatorname{Mult}_{\mathrm{KS}}\left(\left\{D, C_{M}\right\}, C_{M}\right)$ is homotopy equivalent to $S^{1} \times S^{1}$, it follows that

$$
\begin{aligned}
& H_{0}\left(\operatorname{Mult}_{\mathrm{KS}}\left(\left\{D, C_{M}\right\}, C_{M}\right)\right) \simeq k, \\
& H_{1}\left(\operatorname{Mult}_{\mathrm{KS}}\left(\left\{D, C_{M}\right\}, C_{M}\right)\right) \simeq k \oplus k, \\
& H_{2}\left(\operatorname{Mult}_{\mathrm{KS}}\left(\left\{D, C_{M}\right\}, C_{M}\right)\right) \simeq k,
\end{aligned}
$$

and the other parts are zero.

Let $[0,1] \subset \mathbf{R}$ be the closed interval and let $\phi:[0,1] \times S^{1}=[0,1] \times \mathbf{R} / \mathbf{Z} \rightarrow \mathbf{R} / \mathbf{Z}=S^{1}$ be the continuous map given by $(t, x \bmod \mathbf{Z}) \mapsto x+t \bmod \mathbf{Z}$. We think of $\phi$ as a homotopy from the identity map $S^{1} \rightarrow S^{1}$ to itself. The product $\operatorname{id}_{(0,1)} \times \phi$ determines a homotopy $\bar{\phi}:[0,1] \times$ $(0,1) \times S^{1} \rightarrow(0,1) \times S^{1}$ from the identity map of $(0,1) \times S^{1}$ to itself. Let $r_{1}:[0,1] \times(0,1)^{2} \stackrel{\text { id } \times j_{1}}{\rightarrow}$ $[0,1] \times(0,1) \times S^{1} \stackrel{\bar{\phi}}{\rightarrow}(0,1) \times S^{1}$ be the homotopy from $j_{1}$ to $j_{1}$, see discussion before Theorem 8.2 for the maps $j, j_{1}$, and $j_{2}$. Let $r_{2}:[0,1] \times(0,1) \times S^{1} \stackrel{\mathrm{pr}}{\rightarrow}(0,1) \times S^{1} \stackrel{j_{2}}{\rightarrow}(0,1) \times S^{1}$ be the trivial homotopy from $j_{2}$ and $j_{2}$. Define

$$
l:=r_{1} \sqcup r_{2}:[0,1] \times\left((0,1)^{2} \sqcup(0,1) \times S^{1}\right) \rightarrow(0,1) \times S^{1} .
$$

We think of $l$ as a homotopy from $j$ to $j$. If we regard $\bar{\phi}$ as a homotopy from the identity to itself in $\operatorname{Mult}_{\mathrm{KS}}\left(\left\{C_{M}\right\}, C_{M}\right)$, it determines an element $e_{B}$ of $H_{1}\left(\operatorname{Mult}_{\mathrm{KS}}\left(\left\{C_{M}\right\}, C_{M}\right)\right)$. If we think of $l$ as a homotopy from $f_{j}$ to itself in $\operatorname{Mult}_{\mathrm{KS}}\left(\left\{D, C_{M}\right\}, C_{M}\right)$, it determines an element $e_{L}$ of $H_{1}\left(\operatorname{Mult}_{\mathrm{KS}}\left(\left\{D, C_{M}\right\}, C_{M}\right)\right)$. The $C_{\bullet}(\mathrm{KS})$-algebra $\left(H H^{\bullet}(A), H H_{\bullet}(A)\right)$ (or equivalently the KS-algebra $\left(\mathcal{H} \mathcal{H}^{\bullet}(A), \mathcal{H} \mathcal{H}_{\bullet}(A)\right)$ in Theorem 7.14) defines $L \in H_{1}\left(\operatorname{Hom}_{k}\left(H H^{\bullet}(A) \otimes_{k}\right.\right.$ $\left.\left.H H_{\bullet}(A), H H_{\bullet}(A)\right)\right)$ for $e_{L}: L$ is defined to be the image of $e_{L}$ under $H_{*}\left(h_{\left(\left\{D, C_{M}\right\}, C_{M}\right)}^{\mathrm{dg}}\right)$. Similarly, $e_{B}$ determines an element $B$ in $H_{1}\left(\operatorname{Hom}_{k}\left(H H_{\bullet}(A), H H_{\bullet}(A)\right)\right)$.

The morphism $f_{j}$ arising from $j=j_{1} \sqcup j_{2}$ is a generator of the 0 -th homology group. The image of $f_{j}$ under $h_{\left(\left\{D, C_{M}\right\}, C_{M}\right)}^{\mathrm{dg}}$ can be identified with $u: \mathcal{H}^{\bullet}(A) \otimes \mathcal{H} \mathcal{H} \bullet(A) \rightarrow \mathcal{H} \mathcal{H}_{\bullet}(A)$ which is equivalent to the contraction morphism, cf. Theorem 8.2. By a comparison result of Hoyois [18, Theorem 2.3], $B$ in $H_{1}\left(\operatorname{Hom}_{k}\left(H H^{\bullet}(A), H H_{\bullet}(A)\right)\right)$ may be viewed as the Connes operator: if $\mathrm{A}$ is an associative (cofibrant) differential graded algebra which represents $A$ through $\operatorname{Alg}_{\mathrm{As}}\left(\operatorname{Mod}_{k}^{\otimes}\right) \simeq \operatorname{Alg}_{\mathrm{As}}\left(\operatorname{Comp}(k)\left[W^{-1}\right]\right)$, the classical Connes operator $b: B_{\bullet}^{\mathrm{dg}}(\mathrm{A}) \otimes_{\mathrm{A}^{e}} \mathrm{~A} \rightarrow$ $B_{\bullet}^{\operatorname{dg}}(\mathrm{A}) \otimes_{\mathrm{A}^{e}} \mathrm{~A}$ (see, e.g., $[18,24]$ ) is equivalent to $B$, up to the multiplication by \pm 1 , through $B_{\bullet}^{\mathrm{dg}}(\mathrm{A}) \otimes_{\mathrm{A}^{e}} \mathrm{~A} \simeq \mathcal{H} \mathcal{H}_{\bullet}(A) \simeq H_{\bullet}(A)$.

The homotopy $l$ and the composition $\bar{\phi} \circ\left(\operatorname{id}_{[0,1]} \times j\right):[0,1] \times\left((0,1)^{2} \sqcup(0,1) \times S^{1}\right) \rightarrow(0,1) \times S^{1}$ generate the $k$-vector space $k \oplus k \simeq H_{1}\left(\operatorname{Mult}_{\mathrm{KS}}\left(\left\{D, C_{M}\right\}, C_{M}\right)\right)$. Let 1 denote the element of $H_{0}\left(\operatorname{Hom}_{k}\left(\mathcal{H H}^{\bullet}(A), \mathcal{H} \mathcal{H}^{\bullet}(A)\right)\right)$ that corresponds to the identity morphism. By relations of homotopies, we see that

$$
L=B \circ u+u \circ(\mathbf{1} \otimes(-B))=B \circ u-u \circ(\mathbf{1} \otimes B)
$$

in $H_{1}\left(\operatorname{Hom}_{k}\left(H H^{\bullet}(A) \otimes H_{\bullet}(A), H_{\bullet}(A)\right)\right)$. Here "o" indicates the composition. This relation is known as Cartan homotopy/magic formula. In the dg setting over $k$, the shifted complex $H H^{\bullet}(A)[1]$ inherits the structure of an $L_{\infty}$-algebra, i.e., an algebra over a (cofibrant) Lie operad in $\operatorname{Comp}(k)$, from the $\mathbf{E}_{2}$-algebra structure on $\mathcal{H H}^{\bullet}(A) \simeq H H^{\bullet}(A)$. The morphism

$$
L: H H^{\bullet}(A)[1] \otimes H_{\bullet}(A) \rightarrow H_{\bullet}(A)
$$

induced by $L$ appears as the Lie algebra action morphism on $H H_{\bullet}(A)$ (see, e.g., [11]). Since $u$ and $B$ can be explicitly described ( $B$ is equivalent to Connes' operator), thus $L$ also has an explicit presentation. Finally, $H_{2}\left(\operatorname{Mult}_{K S}\left(\left\{D, C_{M}\right\}, C_{M}\right)\right)$ is generated by $B \circ u \circ(\mathbf{1} \otimes B)$. 


\section{Equivariant context}

Our construction in Theorem 7.14 can easily be generalized to an equivariant setting: Let $G$ be a group object in $\mathcal{S}$ and $B G \in \mathcal{S}$ the classifying space. Let $\mathcal{C} \in \mathcal{S} \mathrm{t}_{R}$, that is, a small stable $R$-lienar idempotent-complete $\infty$-category. Suppose that $G$ acts on $\mathcal{C}$, i.e., an left action on $\mathcal{C}$. Namely, $\mathcal{C}$ is an object of $\operatorname{Fun}\left(B G, \mathcal{S} \mathrm{t}_{R}\right)$ whose image under the forgetful functor $\operatorname{Fun}\left(B G, \mathcal{S} \mathrm{t}_{R}\right) \rightarrow \mathcal{S} \mathrm{t}_{R}$ is $\mathcal{C}$. In this setting, we have

Theorem 9.1. The pair $\left(\mathcal{H} \mathcal{H}^{\bullet}(\mathcal{C}), \mathcal{H} \mathcal{H} \bullet(\mathcal{C})\right)$ of Hochschild cohomology and homology $R$-module spectra has the structure of a $\mathbf{K S}$-algebra in $\operatorname{Fun}\left(B G, \operatorname{Mod}_{R}\right)$. In other words, $\left(\mathcal{H} \mathcal{H}^{\bullet}(\mathcal{C}), \mathcal{H} \mathcal{H} \bullet(\mathcal{C})\right)$ is promoted to $\operatorname{Alg}_{\mathbf{K S}}\left(\operatorname{Fun}\left(B G, \operatorname{Mod}_{R}\right)\right)$.

Remark 9.2. The forgetful functor $\operatorname{Alg}_{\mathbf{K S}}\left(\operatorname{Fun}\left(B G, \operatorname{Mod}_{R}\right)\right) \rightarrow \operatorname{Alg}_{\mathbf{K S}}\left(\operatorname{Mod}_{R}\right)$ sends the KSalgebra in Theorem 9.1 to a $\mathbf{K S}$-algebra equivalent to the KS-algebra constructed in Theorem 7.14 .

Theorem 9.1 follows from the following:

Construction 9.3. The construction is almost the same as that of Theorem 7.14. Thus, we highlight necessary modifications.

(i) Let $\mathcal{D}:=\operatorname{Ind}(\mathcal{C})$ be the Ind-category which is an $R$-linear compactly generated $\infty$-category. In particular, $\mathcal{D}$ belongs to $\operatorname{Pr}_{R}^{\mathrm{L}}$. Since $\mathcal{C} \mapsto \operatorname{Ind}(\mathcal{C})$ is functorial, the left action of $G$ on $\mathcal{C}$ induces a left action on $\mathcal{D}$. Namely, $\mathcal{D}$ is promoted to $\operatorname{Fun}\left(B G, \operatorname{Pr}_{R}^{\mathrm{L}}\right)$. The functor category Fun $\left(B G, \operatorname{Pr}_{R}^{\mathrm{L}}\right)$ inherits a (pointwise) symmetric monoidal structure from that of $\operatorname{Pr}_{R}^{\mathrm{L}}$. Let $\operatorname{Mor}_{R}^{G}(\mathcal{D}, \mathcal{D})$ be an internal hom object in the symmetric monoidal $\infty$-category Fun $\left(B G, \operatorname{Pr}_{R}^{\mathrm{L}}\right)$. This is explicitly described as follows: The internal hom object $\mathcal{M o r}_{R}(\mathcal{D}, \mathcal{D})$ (Lemma 5.1) in $\operatorname{Pr}_{R}^{\mathrm{L}}$ has the left action of $G^{\mathrm{op}} \times G$ induced by the functoriality of the internal hom object and the action of $G$ on $\mathcal{D}$ (here $G^{\text {op }}$ denotes the opposite group). The homomorphism $G \rightarrow G^{\text {op }} \times G$ informally given by $g \mapsto\left(g^{-1}, g\right)$ determines a left action of $G$ on $\mathcal{M o r}_{R}(\mathcal{D}, \mathcal{D})$. By the universal property of $\mathcal{M o r}_{R}(\mathcal{D}, \mathcal{D}), \mathcal{M o r}_{R}(\mathcal{D}, \mathcal{D})$ endowed with the $G$-action is an internal hom object from $\mathcal{D}$ to $\mathcal{D}$ in $\operatorname{Fun}\left(B G, \operatorname{Pr}_{R}^{\mathrm{L}}\right)$. As in Lemma $5.1, \mathcal{M o r}_{R}^{G}(\mathcal{D}, \mathcal{D})$ is promoted to $\operatorname{Alg}_{A s}\left(\operatorname{Fun}\left(B G, \operatorname{Mod}_{R}\right)\right) \simeq \operatorname{Fun}\left(B G, \operatorname{Alg}_{\text {As }}\left(\operatorname{Mod}_{R}\right)\right)$. Here the equivalence follows from the definition of the pointwise symmetric monoidal strucutre on $\operatorname{Fun}\left(B G, \operatorname{Mod}_{R}\right)$ [25, Remark 2.1.3.4].

(ii) Recall the adjunction $I: \operatorname{Alg}_{\mathrm{As}_{\mathrm{s}}}\left(\operatorname{Mod}_{R}\right) \rightleftarrows\left(\operatorname{Pr}_{R}^{\mathrm{L}}\right)_{\operatorname{Mod}_{R} /}: E$ induces an adjunction

$$
\operatorname{Alg}_{\mathbf{E}_{2}}\left(\operatorname{Mod}_{R}\right) \simeq \operatorname{Alg}_{\mathrm{As}}\left(\operatorname{Alg}_{\mathrm{As}}\left(\operatorname{Mod}_{R}\right)\right) \rightleftarrows \operatorname{Alg}_{\mathrm{As}}\left(\operatorname{Pr}_{R}^{\mathrm{L}}\right) \simeq \operatorname{Alg}_{\mathbf{E}_{1}}\left(\operatorname{Pr}_{R}^{\mathrm{L}}\right)
$$

(see Section 5). Applying $\operatorname{Fun}(B G,-)$ to this adjunction we get an adjunction

$$
\operatorname{Fun}\left(B G, \operatorname{Alg}_{\mathbf{E}_{2}}\left(\operatorname{Mod}_{R}\right)\right) \rightleftarrows \operatorname{Fun}\left(B G, \operatorname{Alg}_{\mathrm{As}}\left(\operatorname{Pr}_{R}^{\mathrm{L}}\right)\right) \simeq \operatorname{Alg}_{\mathrm{As}}\left(\operatorname{Fun}\left(B G, \operatorname{Pr}_{R}^{\mathrm{L}}\right)\right) .
$$

We define the Hochschild cohomology $R$-module spectrum

$$
\mathcal{H H}^{\bullet}(\mathcal{C}):=\mathcal{H H}^{\bullet}(\mathcal{D}) \in \operatorname{Fun}\left(B G, \operatorname{Alg}_{\mathbf{E}_{2}}\left(\operatorname{Mod}_{R}\right)\right) \simeq \operatorname{Alg}_{\mathbf{E}_{2}}\left(\operatorname{Fun}\left(B G, \operatorname{Mod}_{R}\right)\right)
$$

to be the image of $\mathcal{M o r}_{R}^{G}(\mathcal{D}, \mathcal{D})$ under the right adjoint. For ease of notation, we write $A$ for $\mathcal{H H}^{\bullet}(\mathcal{C})$. Write $\operatorname{RMod}_{A}^{G, \otimes}$ for the image of $A=\mathcal{H} \mathcal{H}^{\bullet}(\mathcal{C})=\mathcal{H} \mathcal{H}^{\bullet}(\mathcal{D}) \in \operatorname{Fun}\left(B G, \operatorname{Alg}_{\mathbf{E}_{2}}\left(\operatorname{Mod}_{R}\right)\right)$

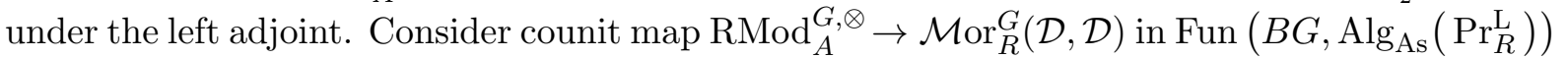
which we regard as a $G$-equivariant associative monoidal functor. As in the non-equivariant case, the natural action of $\mathcal{M o r}_{R}^{G}(\mathcal{D}, \mathcal{D})$ on $\mathcal{D}$ gives rise to a left action of $\operatorname{RMod}_{A}^{G, \otimes}$ on $\mathcal{D}$. More precisely, $\mathcal{D}$ is promoted to an object of $\operatorname{LMod}_{\operatorname{RMod}_{A}^{G, \otimes}}\left(\operatorname{Fun}\left(B G, \operatorname{Pr}_{R}^{\mathrm{L}}\right)\right)$. Let $\operatorname{RPerf}_{A}^{G, \otimes}$ be the symmetric monoidal full subcategory spanned by compact objects. By definition, $\operatorname{RPerf}_{A}^{G, \otimes}$ is 
the associative monoidal small $R$-linear $\infty$-category $\operatorname{RPerf}_{A}^{\otimes}$ endowed with the $G$-action given informally by the $G$-action on $A$. As in Proposition 7.17, the restriction exhibits $\mathcal{C}$ as a left $\operatorname{RPerf}_{A}^{G, \otimes}$-module, that is, an object of $\operatorname{LMod}_{\mathrm{RPerf}_{A}^{G, \otimes}}\left(\operatorname{Fun}\left(B G, \mathcal{S} \mathrm{t}_{R}\right)\right)$.

(iii) Let $\mathcal{H H}_{\bullet}(-): \mathcal{S t}_{R} \rightarrow \operatorname{Fun}\left(B S^{1}, \operatorname{Mod}_{R}\right)$ denote the symmetric monoidal functor which carries $\mathcal{E}$ to $\mathcal{H} \mathcal{H} .(\mathcal{E})$ (see Definition 6.14). Applying $\operatorname{Fun}(B G,-)$ to it, we have a symmetric monoidal functor $\operatorname{Fun}\left(B G, \mathcal{S} \mathrm{t}_{R}\right) \rightarrow \operatorname{Fun}\left(B G, \operatorname{Fun}\left(B S^{1}, \operatorname{Mod}_{R}\right)\right)$. We define $\mathcal{H} \mathcal{H}_{\bullet}(\mathcal{C})$ to be the image of $\mathcal{C}$ in $\operatorname{Fun}\left(B G, \operatorname{Fun}\left(B S^{1}, \operatorname{Mod}_{R}\right)\right)$. By the induced functor

$$
\operatorname{LMod}_{\operatorname{RPerf}_{A}^{G, \otimes}}\left(\operatorname{Fun}\left(B G, \mathcal{S} t_{R}\right)\right) \rightarrow \operatorname{LMod}_{\mathcal{H}} \cdot\left(\operatorname{RPerf}_{A}^{G, \otimes}\right)\left(\operatorname{Fun}\left(B G, \operatorname{Fun}\left(B S^{1}, \operatorname{Mod}_{R}\right)\right)\right),
$$

we regard $\mathcal{H}_{\bullet}(\mathcal{C})$ as a left $\mathcal{H}_{\bullet}\left(\operatorname{RPerf}_{A}^{G, \otimes}\right)$-module. That is to say, $\mathcal{H}_{\bullet}(\mathcal{C})$ is an object of

$$
\begin{aligned}
& \operatorname{LMod}_{\mathcal{H} \mathcal{H} \bullet\left(\operatorname{RPerf}_{A}^{G, \otimes}\right)}\left(\operatorname{Fun}\left(B G, \operatorname{Fun}\left(B S^{1}, \operatorname{Mod}_{R}\right)\right)\right) \\
& \simeq \operatorname{LMod}_{\mathcal{H} \mathcal{H}}\left(\operatorname{RPerf}_{A}^{G, \otimes}\right) \\
& \left.\quad \operatorname{Fun}\left(B S^{1}, \operatorname{Fun}\left(B G, \operatorname{Mod}_{R}\right)\right)\right) .
\end{aligned}
$$

According to the Morita invariance, $\mathcal{H H}_{\bullet}\left(\operatorname{RPerf}_{A}^{G, \otimes}\right)$ can naturally be identified with $\mathcal{H}_{\bullet}(A)$, where $\mathcal{H} \mathcal{H} \bullet(A)$ comes equipped with a left action of $G$ induced by the $G$-action on $A=\mathcal{H} \mathcal{H}^{\bullet}(\mathcal{C})$. Let $i_{!}(A): \mathbf{D C y l} \rightarrow \operatorname{Fun}\left(B G, \operatorname{Mod}_{R}\right)^{\otimes}$ denote the operadic left Kan extension of the map of $\infty$ operads $A: \mathbf{E}_{2}^{\otimes} \rightarrow \operatorname{Fun}\left(B G, \operatorname{Mod}_{R}\right)^{\otimes}$ over $\Gamma$ along $i: \mathbf{E}_{2}^{\otimes} \rightarrow \mathbf{D C y l}$ where $\operatorname{Fun}\left(B G, \operatorname{Mod}_{R}\right)^{\otimes} \rightarrow \Gamma$ is the pointwise symmetric monoidal $\infty$-category induced by the structure on $\operatorname{Mod}_{R}^{\otimes}$. Let $i_{!}(A)_{C}$ be the restriction to $\mathbf{C y l} \subset \mathbf{D C y l}$ which we think of as an object of $\operatorname{Alg}_{\mathbf{C y l}}\left(\operatorname{Fun}\left(B G, \operatorname{Mod}_{R}\right)\right)$. If we replace $\operatorname{Mod}_{R}$ by $\operatorname{Fun}\left(B G, \operatorname{Mod}_{R}\right)$ in the proof of Proposition 7.11 , the argument yields a canonical equivalence $\mathcal{H} \mathcal{H}_{\bullet}(A) \simeq i_{!}(A)_{C}$ in $\operatorname{Fun}\left(B S^{1}, \operatorname{Fun}\left(B G, \operatorname{Mod}_{R}\right)\right)$. Then $A=\mathcal{H}^{\bullet}(\mathcal{C}) \in$ $\operatorname{Alg}_{\mathbf{E}_{2}}\left(\operatorname{Fun}\left(B G, \operatorname{Mod}_{R}\right)\right)$, the left $\mathcal{H} \mathcal{H}_{\bullet}\left(\operatorname{RPerf}_{A}^{G, \otimes}\right)$-module $\mathcal{H} \mathcal{H} \bullet(\mathcal{C})$, and $\mathcal{H} \mathcal{H} \bullet(A) \simeq i_{!}(A)_{C}$ determine an object of

$$
\operatorname{Alg}_{\mathbf{E}_{2}}\left(\operatorname{Fun}\left(B G, \operatorname{Mod}_{R}\right)\right) \times{ }_{\operatorname{Alg}_{\mathrm{As}}\left(\operatorname{Fun}\left(B S^{1}, \operatorname{Fun}\left(B G, \operatorname{Mod}_{R}\right)\right)\right)} \operatorname{LMod}\left(\operatorname{Fun}\left(B S^{1}, \operatorname{Fun}\left(B G, \operatorname{Mod}_{R}\right)\right)\right),
$$

which is naturally equivalent to $\operatorname{Alg}_{\mathbf{K S}}\left(\operatorname{Fun}\left(B G, \operatorname{Mod}_{R}\right)\right.$ ) (see Corollary 4.21). Consequently, we obtain a KS-algebra in $\operatorname{Fun}\left(B G, \operatorname{Mod}_{R}\right)$ having the property described in Remark 9.2.

Remark 9.4. The argument in Construction 9.3 can be applied to show other functorialities. For example, suppose that we are given an equivalence $f: \mathcal{C}_{1} \stackrel{\sim}{\rightarrow} \mathcal{C}_{2}$ in $\mathcal{S t}_{R}$. Then it gives rise to an equivalence

$$
\left(\mathcal{H}^{\bullet}\left(\mathcal{C}_{1}\right), \mathcal{H} \mathcal{H}_{\bullet}\left(\mathcal{C}_{1}\right)\right) \stackrel{\sim}{\longrightarrow}\left(\mathcal{H H}^{\bullet}\left(\mathcal{C}_{2}\right), \mathcal{H} \mathcal{H}_{\bullet}\left(\mathcal{C}_{2}\right)\right)
$$

in $\operatorname{Alg}_{\mathbf{K S}}\left(\operatorname{Mod}_{R}\right)$. To see this, consider the $\infty$-category $I$ which consists of two objects $\{1,2\}$ such that for any $i, j \in\{1,2\}$, the mapping $\operatorname{space}^{\operatorname{Map}_{I}}(i, j)$ is a contractible space (so that $I$ is (the nerve of) an ordinary groupoid). The equivalence $f$ amounts to a functor $I \rightarrow \mathcal{S} \mathrm{t}_{R}$ which carries 1 and 2 to $\mathcal{C}_{1}$ and $\mathcal{C}_{2}$, respectively, and carries the unique morphism $1 \rightarrow 2$ to $f$. Namely, we have an object of $\operatorname{Fun}\left(I, \mathcal{S} \mathrm{t}_{R}\right)$ so that the construction of Ind-categories gives rise to an object $F \in \operatorname{Fun}\left(I, \operatorname{Pr}_{R}^{\mathrm{L}}\right)$. Now apply the argument in Construction 9.3 by replacing $B G$ with $I$ : Namely, we first consider the internal hom object from $F$ to $F$ in $\operatorname{Fun}\left(I, \operatorname{Pr}_{R}^{\mathrm{L}}\right)$. Note that by $[27$, Corollary 3.3.3.2] and the fact that $I$ is a Kan complex (i.e., $\infty$-groupoid), Fun $\left(I, \operatorname{Pr}_{R}^{\mathrm{L}}\right)$ can be identified with a limit of the constant diagram $I \rightarrow \widehat{\operatorname{Cat}}_{\infty}$ with value $\operatorname{Pr}_{R}^{\mathrm{L}}$. There exists an internal hom object from $F$ to $F$ that is given by the tensor product $F \otimes F^{\vee}$ of $F$ and its dual object $F^{\vee}$, where $F^{\vee}$ is given by taking termwise dual objects. According to [25, Corollary 4.7.1.40], $F \otimes F^{\vee}$ is promoted to an endomorphism algebra $\mathcal{E} \operatorname{nd}(F) \in \operatorname{Alg}_{\text {As }}\left(\operatorname{Fun}\left(I, \operatorname{Pr}_{R}^{\mathrm{L}}\right)\right)$ equipped with a left action on $F$ in an essentially unique way. The evaluation at each $1,2 \in I$ gives us the endomorphism algebras $\mathcal{E} \operatorname{nd}_{R}\left(\operatorname{Ind}\left(\mathcal{C}_{1}\right)\right)$ and $\mathcal{E} \operatorname{nd}_{R}\left(\operatorname{Ind}\left(\mathcal{C}_{2}\right)\right)$, respectively. Thus, replacing $B G$ with $I$ in the procedure in Construction 9.3, we obtain an object in $\operatorname{Alg}_{\mathbf{K S}}\left(\operatorname{Fun}\left(I, \operatorname{Mod}_{R}\right)\right) \simeq \operatorname{Fun}\left(I, \operatorname{Alg}_{\mathbf{K S}}\left(\operatorname{Mod}_{R}\right)\right)$, 
which brings us an induced equivalence $\left(\mathcal{H} \mathcal{H}^{\bullet}\left(\mathcal{C}_{1}\right), \mathcal{H} \mathcal{H}_{\bullet}\left(\mathcal{C}_{1}\right)\right) \stackrel{\sim}{\rightarrow}\left(\mathcal{H} \mathcal{H}^{\bullet}\left(\mathcal{C}_{2}\right), \mathcal{H} \mathcal{H}_{\bullet}\left(\mathcal{C}_{2}\right)\right)$. Finally, we remark that this argument can be applied to any diagram $K \rightarrow \mathcal{S} \mathrm{t}_{R}$ such that $K$ is a Kan complex (i.e., an $\infty$-groupoid). That is, if we are given a diagram/functor $P: K \rightarrow \mathcal{S} \mathrm{t}_{R}$ from an $\infty$-groupoid $K$, it gives rise to a diagram $P_{\mathbf{K S}}: K \rightarrow \operatorname{Alg}_{\mathbf{K S}}\left(\operatorname{Mod}_{R}\right)$ of $\mathbf{K S}$-algebras such that for any $k \in K, P_{\mathbf{K S}}(k)$ is equivalent to the $\mathbf{K S}$-algebra constructed from $P(k) \in \mathcal{S t}_{R}$ in Theorem 7.14.

\section{Acknowledgements}

The author would like to thank Takuo Matsuoka for valuable and inspiring conversations related to the subject of this paper. He would like to thank everyone who provided constructive feedback at his talks about the main content of this paper. He would also like to thank the referees for their useful suggestions. This work is supported by JSPS KAKENHI grant $17 \mathrm{~K} 14150$.

\section{References}

[1] Armenta M.A., Keller B., Derived invariance of the Tamarkin-Tsygan calculus of an algebra, C. R. Math. Acad. Sci. Paris 357 (2019), 236-240, arXiv:1811.04440.

[2] Ayala D., Francis J., Factorization homology of topological manifolds, J. Topol. 8 (2015), 1045-1084, arXiv:1206.5522.

[3] Barthel T., May J.P., Riehl E., Six model structures for DG-modules over DGAs: model category theory in homological action, New York J. Math. 20 (2014), 1077-1159, arXiv:1310.1159.

[4] Batanin M.A., The Eckmann-Hilton argument and higher operads, Adv. Math. 217 (2008), 334-385, arXiv:math.CT/0207281.

[5] Ben-Zvi D., Francis J., Nadler D., Integral transforms and Drinfeld centers in derived algebraic geometry, J. Amer. Math. Soc. 23 (2010), 909-966, arXiv:0805.0157.

[6] Blumberg A.J., Gepner D., Tabuada G., A universal characterization of higher algebraic K-theory, Geom. Topol. 17 (2013), 733-838, arXiv:1001.2282.

[7] Blumberg A.J., Gepner D., Tabuada G., Uniqueness of the multiplicative cyclotomic trace, Adv. Math. 260 (2014), 191-232, arXiv:1103.3923.

[8] Blumberg A.J., Mandell M.A., Localization theorems in topological Hochschild homology and topological cyclic homology, Geom. Topol. 16 (2012), 1053-1120, arXiv:0802.3938.

[9] Cohn L., Differential graded categories are $K$-linear stable $\infty$-categories, arXiv:1308.2587.

[10] Connes A., Cohomologie cyclique et foncteurs Ext ${ }^{n}$, C. R. Acad. Sci. Paris Sér. I Math. 296 (1983), 953-958.

[11] Dolgushev V., Tamarkin D., Tsygan B., Formality of the homotopy calculus algebra of Hochschild (co)chains, arXiv:0807.5117.

[12] Dolgushev V., Tamarkin D., Tsygan B., Formality theorems for Hochschild complexes and their applications, Lett. Math. Phys. 90 (2009), 103-136, arXiv:0901.0069.

[13] Dwyer W.G., Kan D.M., Function complexes in homotopical algebra, Topology 19 (1980), 427-440.

[14] Getzler E., Jones J.D.S., The cyclic homology of crossed product algebras, J. Reine Angew. Math. 445 (1993), 161-174.

[15] Hinich V., Rectification of algebras and modules, Doc. Math. 20 (2015), 879-926, arXiv:1311.4130.

[16] Horel G., Factorization homology and calculus à la Kontsevich Soibelman, J. Noncommut. Geom. 11 (2017), 703-740, arXiv:1307.0322.

[17] Hovey M., Shipley B., Smith J., Symmetric spectra, J. Amer. Math. Soc. 13 (2000), 149-208, arXiv:math.AT/9801077.

[18] Hoyois M., Homotopy fixed points of the circle action Hochschild hoology, arXiv:1506.07123.

[19] Iwanari I., Kodaira-Spencer morphisms, Hochschild invariants and D-modules, Preprint.

[20] Iwanari I., Period mappings for noncommutative algebras, arXiv:1604.08283.

[21] Joyal A., Quasi-categories and Kan complexes, J. Pure Appl. Algebra 175 (2002), 207-222. 
[22] Kock J., Toën B., Simplicial localization of monoidal structures, and a non-linear version of Deligne's conjecture, Compos. Math. 141 (2005), 253-261, arXiv:math.AT/0304442.

[23] Kontsevich M., Soibelman Y., Notes on $A_{\infty}$-algebras, $A_{\infty}$-categories and non-commutative geometry, in Homological Mirror Symmetry, Lecture Notes in Phys., Vol. 757, Springer, Berlin, 2009, 153-219, arXiv:math.RA/0606241.

[24] Loday J.-L., Cyclic homology, Grundlehren der Mathematischen Wissenschaften, Vol. 301, Springer-Verlag, Berlin, 1992.

[25] Lurie J., Higher algebra, Preprint, 2017, available at http://people.math.harvard.edu/ lurie/papers/ HA.pdf.

[26] Lurie J., Rotation invariance in algebraic K-theory, Preprint.

[27] Lurie J., Higher topos theory, Annals of Mathematics Studies, Vol. 170, Princeton University Press, Princeton, NJ, 2009.

[28] Muro F., Dwyer-Kan homotopy theory of enriched categories, J. Topol. 8 (2015), 377-413, arXiv:1201.1575.

[29] Schwede S., Shipley B., Stable model categories are categories of modules, Topology 42 (2003), 103-153, arXiv:math.AT/0108143.

[30] Schwede S., Shipley B.E., Algebras and modules in monoidal model categories, Proc. London Math. Soc. 80 (2000), 491-511, arXiv:math.AT/9801082.

[31] Shipley B., Symmetric spectra and topological Hochschild homology, K-Theory 19 (2000), 155-183, arXiv:math.AT/9801079.

[32] Shipley B., A convenient model category for commutative ring spectra, in Homotopy Theory: Relations with Algebraic Geometry, Group Cohomology, and Algebraic K-Theory, Contemp. Math., Vol. 346, Amer. Math. Soc., Providence, RI, 2004, 473-483.

[33] Tabuada G., Topological Hochschild and cyclic homology for differential graded categories, arXiv:0804.2791.

[34] Thomas J., Kontsevich's Swiss cheese conjecture, Geom. Topol. 20 (2016), 1-48, arXiv:1011.1635. 